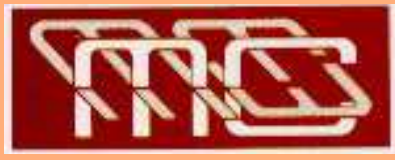

\title{
The Eigenvalue Problem in Linear Viscoelastic Structures: New Numerical Approaches and the Equivalent Viscous Model
}

Ph.D. Thesis subimtted by

Mario Lázaro Navarro

Ingeniero de Caminos, Canales y Puertos

Licenciado en Ciencias Matemáticas

Advisor

Prof. Dr. José Luis Pérez Aparicio

Departamento de Mecánica de los Medios Continuos y

Teoría de Estructuras

UNIVERSITAT POLITÈCNICA VALÈNCIA

Valencia - April, 2013 

Esta tesis está dedicada a Pili, que la vio nacer, y a Marcos y Gonzalo, que crecieron con ella. 



\section{Declaración inicial}

La presente tesis doctoral se ha redactado como un compendio de seis artículos de investigación enviados a revistas indexadas en el JCR. De ellos dos se encuentran ya publicados mientras que el resto está en fase de revisión. De acuerdo a la "Normativa Interna del Departamento de Mecáncia de los Medios Continuos y Teoría de Estructuras sobre la elaboración y defensa de Tesis Doctorales como compendio de publicaciones (Mayo de 2012)" se detallan a continuación las referencias de los artículos que forman el cuerpo principal del documento (tanto los publicados como los que se encuentran en fase de revisión).

Mario Lázaro, José L. Pérez Aparicio, Marcelo Epstein. Computation of eigenvalues in proportionally damped viscoelastic structures based on the fixed-point iteration. Applied Mathematics and Computation, 2012, 219(8), pp 3511-3529.

Mario Lázaro, José L. Pérez Aparicio. Characterization of Real Eigenvalues in Linear Viscoelastic Oscillators and the Non-viscous Set. Submitted to Journal of Applied Mechanics, transactions of the ASME, paper ID JAM12-1529, under review.

Mario Lázaro, José L. Pérez Aparicio. Parametric solutions of the eigenvalue problem for single-degree-of-freedom viscoelastic systems. Submitted to AIAA Journal, paper ID 2012-03-J051941, under review

Mario Lázaro, José L. Pérez Aparicio. Multiparametric Computation of Eigenvalues for Linear Viscoelastic Structures. Computers \& Structures, 2013, 117, pp 67-81.

Mario Lázaro, José L. Pérez Aparicio, Marcelo Epstein An Equivalent Viscous Model for Linear Viscoelastic System. Submitted to Mechanical Systems and Signal Processing, paper ID MSSP12-63, under review.

Mario Lázaro, José L. Pérez Aparicio. Dynamic analysis of frame structures with free viscoelastic layers: New closed-form solutions of eigenvalues and a viscous approach. Submitted to Engineering Structures, paper ID ENGSTRUCT-D-12-01352, under review. 


\section{Abstract}

The analysis and vibration control is particularly important in many branches of engineering, especially mechanical, civil, aeronautics and automotive. This is so up to the point that almost this analysis is identified as a separate area within the dynamic analysis of structures. Since the beginning, dissipative or damping forces have been one of the most difficult to be represented. The viscous model, due to its simplicity and versatility, has been and still is the great paradigm of damping models. However, due to the introduction of memory materials in practical applications, it was necessary to improve the model to consider the viscoelasticity phenomenon; the viscoelasticity, although closely related to the speed of response, required the introduction of the so-called hereditary functions. These functions allow us to express the dissipative forces not only as functions of the instantaneous velocity, but also of the velocity history, from the beginning of the movement hence the term memory - Naturally, such theoretical advance into the model is also accompanied by computational disadvantages. In fact, before the response was obtained as solution of a system of linear differential equations and now a a system of integro-differential equations must be solved.

Analysis of the free vibrations of the viscoelastic damping systems leads to a nonlinear eigenvalue problem, the main feature of which is a frequency-dependent damping matrix. The study of the solution of eigenvalues and eigenvectors of this problem becomes of special importance in order to know the structural modes of vibration or if the frequency-domain response is sought. The eigenvalue set is formed by complex-conjugate pairs and a real eigenvalues, the number of which depends on the mathematical form of the damping function.

The main objectives of this Thesis are two: first, to deepen the knowledge of the eigenvalue problem of viscoelastic systems through the proposal of new numerical methods. Second, to develop a new viscous model that, under certain conditions, can reproduce the response of the viscoelastic model accurately enough.

The document is divided into eight chapters, among them, the main body is constituted by Chapters 2 to 7 . All of them are research articles that have either 
been published or are under review in sources included in the Journal Citation Reports (JCR). For this reason, all chapters retain the intrinsic structure of an article, including introduction and bibliography.

The first four chapters (2 to 5 ) are focused on the study of the aforementioned nonlinear eigenvalue problem with two numerical methodologies proposed. The first is an iterative procedure based on the fixed-point scheme, specially developed for proportional or lightly non-proportional systems (those with decoupled or almostuncoupled modes). The second methodology, presented in two separate chapters, is called parametric since the fundamentals are based on the perturbation of certain damping parameter and allows us to construct almost-analytical solutions of the eigenvalues, both for single and multiple degree-of-freedom systems. The study of the eigenvalue problem is completed by a chapter on real eigenvalues, also called non-viscous eigenvalues. To this end, a new mathematical characterization of these eigenvalues is presented and a new concept is introduced: the non-viscous set.

In the last two chapters (6 and 7) an equivalent viscous model is developed. The approach has been proposed as an approximated substitute of the response of viscoelastic systems. The analysis is performed with the study of the transfer function in the frequency domain. In a first stage (Chap 6), the related mathematical nature is analyzed. It is shown that the exact transfer function of a viscoelastic model can be expanded as a sum of a transfer function, characteristic of certain viscous model, plus a residual term. The latter is directly dependent on the induced damping level and on the modal coupling (non-proportionality of the damping matrix). In a second stage (Chap. 7), an real structural application formed by plane frame structures with free damping layers of viscoelastic material is developed. In this chapter the equivalent viscous model and an improved parametric method to compute the eigenvalues are applied, achieving the conjunction of the two objectives of the present Thesis. 


\section{Resumen}

El análisis y el control de las vibraciones cobra especial importancia en muchas ramas de la ingeniería, en especial la ingeniería mecánica, civil, aernonáutica y automovilística. Tal es así que prácticamente se identifica como un area independiente dentro del análisis dinámico de estructuras. Desde los comienzos de esta teoría, las fuerzas disipativas o de amortiguamiento han sido uno de los fenómenos más difíciles de modelizar. El modelo viscoso, por su sencillez y versatilidad ha sido y sigue siendo el gran paradigma de los modelos de amortiguamiento. Sin embargo, como consecuencia de la aparición de materiales con memoria se introdujo el fenómeno de la viscoelasticidad; Ésta, si bien está también íntimmamente ligada a la velocidad de la respuesta, necesitó de la introducción de las denominadas funciones hereditarias, que permiten poner a las fuerzas disipativas como función no solo de la velocidad instantánea sino de la historia de velocidades desde el comienzo del movimiento, de ahí el término memoria. De forma natural, el avance teórico introducido en el modelo supone también una complicación computacional, pues donde antes teníamos un sistema lineal de ecuaciones diferenciales ahora tenemos un sistema de ecuaciones integro-diferenciales.

El análisis de las vibraciones libres de los sistemas con amortiguamiento viscoelástico conduce a un problema nolineal de autovalores donde la característica principal es una matriz de amortiguamiento que depende de la frecuencia de excitación. El estudio de la solución de autovalores y autovectores de este problema es importante si se desean conocer los modos de vibración de la estructura o si se pretende obtener la respuesta en el dominio de la frecuencia del sistema. El objetivo fundamental de esta Tesis Doctoral es doble: Por un lado, profundizar en el conocimiento del problema de autovalores de sistemas viscoelásticos proponiendo para ello nuevos métodos numéricos de resolución. Por otro, desarrollar un nuevo modelo viscoso que, bajo ciertas condiciones, reproduzca la respuesta del modelo viscoelástico con suficiente aproximación.

La Tesis se divide en ocho capítulos, de ellos el cuerpo principal se encuentra en los seis centrales (Capítulos 2 a 7. Todos ellos son artículos de investigación que, o bien han sido publicados, o bien están en proceso de revisión en revistas con- 
tenidas en el Journal Citation Reports (JCR). Por esta razón, todos los capítulos conservan la estructura intrínseca de un artículo, incluídas una introducción y una bibliografía en cada uno.

Los cuatro primeros capítulos (Capítulos 2 a 5) se centran en el estudio del problema no lineal de autovalores. Se proponen dos metodologías de resolución: la primera es un procedimiento iterativo basado en el esquema del punto-fijo y desarrollado para sistemas proporcionales o ligeramente no-proporcionales (aquellos en los que los modos se presentan desacoplados o casi desacoplados). La segunda metodología (presentada en dos capítulos diferentes), denominada parametrica, permite obtener soluciones casi-analíticas de los autovalores, tanto para sistemas de un grado de libertad como para sistemas de múltiples grados de libertad y dentro de éstos, para sistemas proporcionales y no proporcionales. El estudio del problema de autovalores se completa con un capítulo dedicado a los autovalores reales, también denominados autovalores no viscosos. En él se demuestra una nueva caracterización matemática que deben cumplir dichos autovalores y que permite proponer un nuevo concepto: el conjunto no-viscoso.

Los dos últimos capítulos (Capítulos 6 y 7 ) analizan el Modelo Viscoso Equivalente como propuesta para la modelización de la respuesta de sistemas viscoelásticos. El análisis se realiza desde el dominio de la frecuencia estudiando la función de transferencia. En una primera etapa (penúltimo capítulo), de naturaleza más matemática, se demuestra que la función de transferencia exacta de un modelo viscoelástico se puede expresar como suma de una función de transferencia propia de un modelo viscoso más un término denominado residual, directamente dependiente del nivel de amortiguamiento inducido y del acoplamiento modal (noproporcionalidad de la matriz de amortiguamiento). En una segunda etapa (último capítulo), se desarrolla una aplicación para estructuras reales formadas por entramados planos de elementos 1D amortiguados con capas de material viscoelástico. Este tipo de estructuras ha permitido usar una variante mejorada del método paramétrico para la obtención de los autovalores, de forma que en este último capítulo ha servido como nexo de unión de las metodolgías más importantes desarrolladas en la Tesis. 


\section{Resum}

L'anàlisi i el control de les vibracions cobra especial importància en moltes branques de l'enginyeria, especialment l'enginyeria mecànica, civil, aernonáutica i automobilística. Tal és així que pràcticament s'identifica com una àrea independent dins de l'anàlisi dinàmic d'estructures. Des dels començaments d'aquesta teoria, les forces dissipatives o d'amortiment han estat un dels fenòmens més difícils de modelitzar. El model viscós, per la seva senzillesa i versatilitat ha estat i continua sent el gran paradigma dels models d'amortiment. No obstant això, com a conseqüència de l'aparició de materials amb memòria es va introduir el fenomen de la viscoelasticitat, Aquesta, si bé està també íntimmamente lligada a la velocitat de la resposta, va necessitar de la introducció de les anomenades funcions hereditàries, que permeten posar a les forces dissipatives com a funció no només de la velocitat instantània sinó de la història de velocitats des del començament del moviment, d'aquí el terme memòria. De forma natural, l'avanç teòric introduït en el model suposa també una complicació computacional, doncs on abans teníem un sistema lineal d'equacions diferencials ara tenim un sistema d'equacions íntegrediferencials.

L'anàlisi de les vibracions lliures dels sistemes amb esmorteïment viscoelàstic condueix a un problema nolineal de autovalors on la característica principal és una matriu d'amortiment que depèn de la freqüència d'excitació. L'estudi de la solució d'autovalors i autovectors d'aquest problema és important si es volen conèixer les maneres de vibració de l'estructura o si es pretén obtenir la resposta en el domini de la freqüència del sistema. L'objectiu fonamental d'aquesta tesi doctoral és doble: d'una banda, aprofundir en el coneixement del problema d'autovalors de sistemes viscoelàstics proposant per a això nous mètodes numèrics de resolució. De l'altra, desenvolupar un nou model viscós que, sota certes condicions, reprodueixi la resposta del model viscoelàstic amb suficient aproximació.

La Tesi es divideix en vuit capítols, dels quals el cos principal es troba en els sis centrals (capítols 2 a 7). Tots ells són articles d'investigació que, o bé han estat publicats, o bé estan en procés de revisió en revistes contingudes al Journal Citation Reports (JCR). És per això que tots els capítols conserven l'estructura intrínseca d'un article, incloses una introducció i una bibliografia a cada un. 
Els quatre primers capítols (capítols 2 a 5) es centren en l'estudi del problema no lineal de autovalors. Es proposen dues metodologies de resolució: la primera és un procediment iteratiu basat en l'esquema del punt-fix i desenvolupat per a sistemes proporcionals o lleugerament no-proporcionals (aquells en que les maneres es presenten desacoblats o gairebé desacoblats). La segona metodologia (presentada en dos capítols diferents), anomenada paramètrica, permet obtenir solucions quasianalítiques dels autovalors, tant per a sistemes d'un grau de llibertat com per a sistemes de múltiples graus de llibertat i dins d'aquests, per a sistemes proporcionals i no proporcionals. L'estudi del problema d'autovalors es completa amb un capítol dedicat als autovalors reals, també anomenats autovalors no viscosos. En ell es demostra una nova caracterització matemàtica que han de complir aquests autovalors i que permet proposar un nou concepte: el conjunt no-viscós.

Els dos últims capítols (capítols 6 i 7) analitzen el model viscós Equivalent com a proposta per a la modelització de la resposta de sistemes viscoelàstics. L'anàlisi es realitza des del domini de la freqüència estudiant la funció de transferència. En una primera etapa (penúltim capítol), de naturalesa més matemàtica, es demostra que la funció de transferència exacta d'un model viscoelàstic es pot expressar com a suma d'una funció de transferència pròpia d'un model viscós més un terme denominat residual, directament dependent del nivell d'amortiment induït i l'acoblament modal (no-proporcionalitat de la matriu d'amortiment). En una segona etapa (últim capítol), es desenvolupa una aplicació per estructures reals formades per entramats plans d'elements 1D esmorteïts amb capes de material viscoelàstic. Aquest tipus d'estructures ha permès utilitzar una variant millorada del mètode paramètric per a l'obtenció dels autovalors, de manera que en aquest últim capítol ha servit com a nexe d'unió de les metodolgías més importants desenvolupades en la Tesi. 


\section{Acknowledgements}

This dissertation would not have been possible without the guidance and the help of several individuals who in one way or another contributed and extended their valuable assistance in the preparation and completion of this study.

It gives me great pleasure in acknowledging the support and help of my Advisor Prof. Dr. José L. Pérez Aparicio, who has supported me throughout my thesis with patience, experience and knowledge, whilst allowing me the room to work in my own way.

I wish to thank Dr. Marcelo Epstein, it was for me a great honor to work with him during his stay at the Polytechnic University of Valencia between November and December, 2011. It was very rewarding for me to share his experiences and restlessness in computational mechanics and mathematics.

I am also grateful to César Fernández, since he has traveled with me as a partner most of this investigation, we exchanged ideas and shared valuable insights over the years. To Dr. Roberto Palma and Dr. Rafael Bravo, for their valuable advices on the writing and edition of the papers.

This thesis would not have been possible unless the help and support of my parents, Antonio and $\mathrm{M}^{\mathrm{a}}$ Pilar, because they have believed in me since the beginnings. Of course, I must mention to my wife Pili and to my sons, Marcos and Gonzalo. They have always been there in moments of weakness and happiness, in spite of the time I've spent on this work instead of passing them. 



\section{Contents}

Declaración inicial

Abstract

Resumen

Resum

IX

Acknowledgements

XI

Contents

XIII

1 Introduction 1

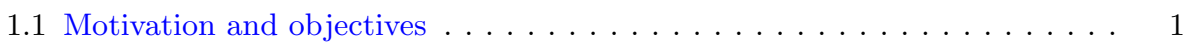

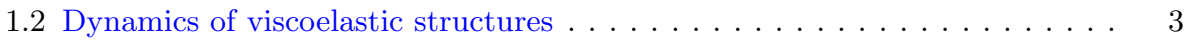

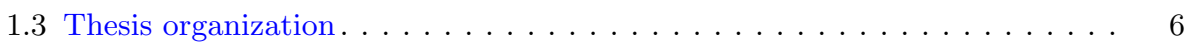

1.3.1 A new recursive scheme to eigenvalues' computation . . . . . . . 7

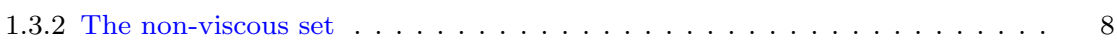

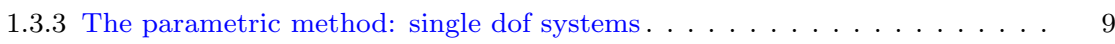

1.3.4 The parametric method: multiple dof systems . . . . . . . . . 10

1.3.5 The Equivalent Viscous Model. . . . . . . . . . . . . . . . . 11

1.3.6 An application to frame structures with free viscoelastic layers . . . . . . 12

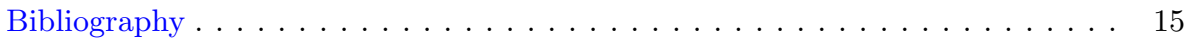


2 Computation of eigenvalues in proportionally damped viscoelastic structures based on the fixed-point iteration

(published in Applied Mathematics and Computation)

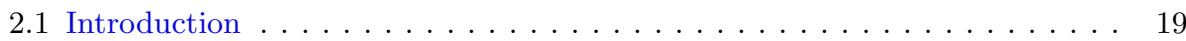

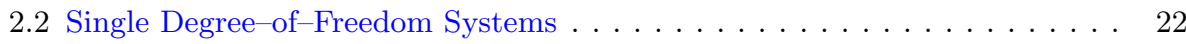

2.2.1 Eigenvalue Problem and Recursive Functions . . . . . . . . . . . . . . 22

2.2 .2 Complex Eigenvalues. . . . . . . . . . . . . . . . . . 25

2.2 .3 Real Eigenvalues . . . . . . . . . . . . . . . . . . . . . . . . 34

2.3 Multiple Degree-of-Freedom Systems . . . . . . . . . . . . . . . 35

2.4 Numerical Examples . . . . . . . . . . . . . . . . . . . . . . . . . . . . 37

2.4.1 Example 1: Single Degree-of-Freedom Systems . . . . . . . . . . . . . 37

2.4.2 Example 2: Multiple Degree-of-Freedom Systems . . . . . . . . . . . . 42

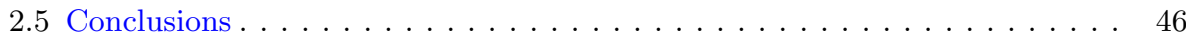

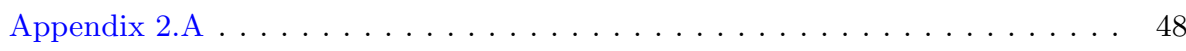

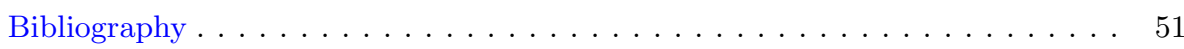

\section{Characterization of Real Eigenvalues in Linear Vis- coelastic Oscillators and the Non-viscous Set (submitted to} Journal of Applied Mechanics, Transactions of the ASME) 57

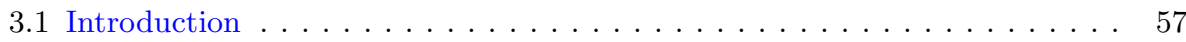

3.2 Location of real eigenvalues; the non-viscous set . . . . . . . . . . . 59

3.2 .1 Exact Limits of the Non-viscous Set . . . . . . . . . . . . . . . 64

3.2 .2 Approximated Limits of the Non-visocus Set. . . . . . . . . . . . 67

3.3 Numerical Computation of Non-viscous Eigenvalues . . . . . . . . . . . . . 69

3.3.1 Existing One-Step Methods. . . . . . . . . . . . . . . . . . . . . . . 69

3.3 .2 Proposed Method. . . . . . . . . . . . . . . . . . . . . . 70

3.4 Numerical Example. . . . . . . . . . . . . . . . . . . . . . . . . . 72

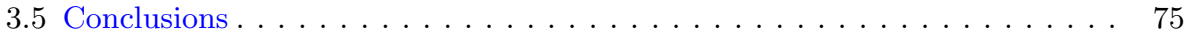

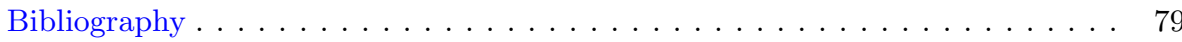

4 Parametric solutions of the eigenvalue problem for singledegree-of-freedom viscoelastic systems (submitted to AIAA Journal)

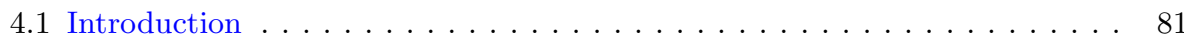


4.2 Dynamics of Single Degree-of-Freedom Viscoelastic Systems . . . . . . . . . 84

4.3 Parametric computation of the eigenvalues . . . . . . . . . . 85

4.3 .1 Fundamentals of the method . . . . . . . . . . . . . . . . 85

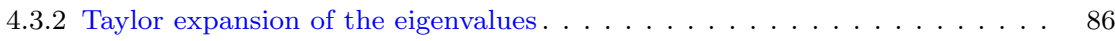

4.3.3 Improving the solution. Eigenvalue differential equation . . . . . . . . . . 87

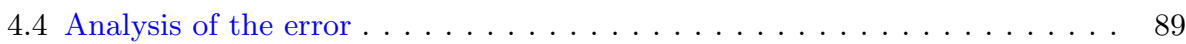

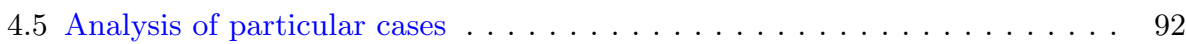

4.5 .1 The viscous model . . . . . . . . . . . . . . . . . . . . 92

4.5.2 The Biot's exponential model . . . . . . . . . . . . . . . . . . . 93

4.5.3 The Biot's multi-exponential model . . . . . . . . . . . . . . . . 100

4.6 Conclusions . . . . . . . . . . . . . . . . . . . . . . . . . . 109

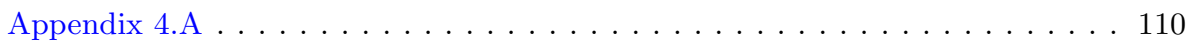

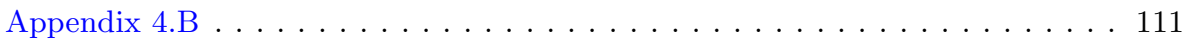

Bibliography . . . . . . . . . . . . . . . . . . . . . 113

\section{Multiparametric Computation of Eigenvalues for Lin-} ear Viscoelastic Structures (published in Computers \&3 Structures) 117

5.1 Introduction . . . . . . . . . . . . . . . . . . . . . . . . . 117

5.2 The Eigenvalue Problem in viscoelastic structures . . . . . . . . . . . . . 120

5.2 .1 The Set of Eigenvalues . . . . . . . . . . . . . . . . . . . . . 120

5.2.2 Parametric formulation of the Eigenvalues . . . . . . . . . . . . 121

5.3 Eigenvalues' Differential Equations . . . . . . . . . . . . . . . . . . . . 122

5.3 .1 Proportional Damping. . . . . . . . . . . . . . . . . . . . . . . . . 123

5.3 .2 Non-proportional Damping. . . . . . . . . . . . . . . . . . . . . . . . . 125

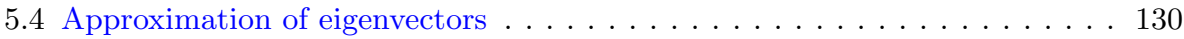

5.4.1 Solution of the ill-conditioned linear system . . . . . . . . . . . . . 130

5.4.2 Approximation for lightly non-proportional damping . . . . . . . . . . . 131

5.4 .3 Approximation for light damping . . . . . . . . . . . . . . . . . . 132

5.5 Analysis of the error and computational complexity . . . . . . . . . . . 132

5.5 .1 Analysis of the error . . . . . . . . . . . . . . . . . . . . . 133

5.5.2 Computational Complexity . . . . . . . . . . . . . . . . . . . . 135

5.6 Numerical Examples . . . . . . . . . . . . . . . . . . . . . . . 136

5.6.1 Example 1. Discrete structures with proportional damping . . . . . . . 136

5.6.2 Example 2. Discrete structures with non-proportional damping . . . . . . . 143

5.6.3 Example 3. Continuous structures with non-proportional damping . . . . . 148 
5.7 Conclusions . . . . . . . . . . . . . . . . . . . . . . . . . . . . . 153

Bibliography . . . . . . . . . . . . . . . . . . . . . . . 155

\section{An Equivalent Viscous Model for Linear Viscoelastic}

Systems (submitted to Mechanical Systems and Signal Processing) 159

6.1 Introduction . . . . . . . . . . . . . . . . . . . . . . . . 159

6.2 Fundamentals of the Proposed Method. . . . . . . . . . . . . . . . . . . . 162

6.2.1 Eigenproblem in Viscoelastic Structures . . . . . . . . . . . . . . . . . 162

6.2.2 The Equivalent Viscous Model. . . . . . . . . . . . . . . . . . . . . . . . 163

6.3 Decomposition of the Transfer Function . . . . . . . . . . . . . . 165

6.3.1 The Transfer Function. . . . . . . . . . . . . . . . . . . . . . . . . . . 165

6.3 .2 Expansion of the complex eigenvectors . . . . . . . . . . . . . . 167

6.3 .3 Expansion of the Residues . . . . . . . . . . . . . . . . . . . 168

6.3 .4 Expansion of the TF . . . . . . . . . . . . . . . . . . . . 170

6.4 Bound of the Residual Transfer Function . . . . . . . . . . . . . . . . . . . . 171

6.4 .1 Indexes of non-proportionality and damping . . . . . . . . . . . . . . 171

6.4 .2 Upper Bound Computation. . . . . . . . . . . . . . . . . . . . . . . . 172

6.5 Numerical Examples . . . . . . . . . . . . . . . . . . . . . . . . . 177

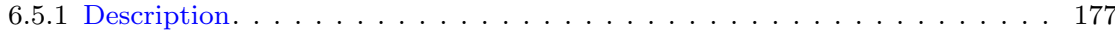

6.6 Conclusions . . . . . . . . . . . . . . . . . . . . . . . . . . 182

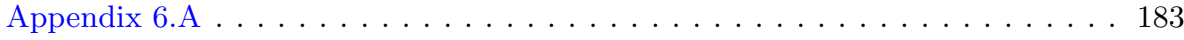

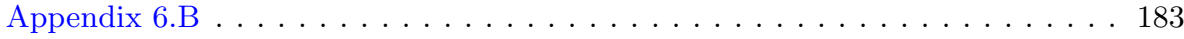

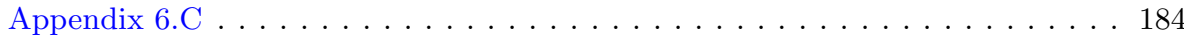

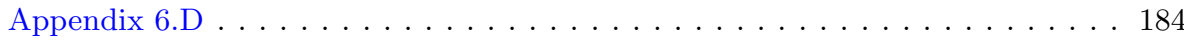

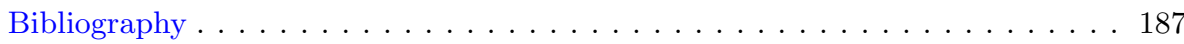

7 Dynamic analysis of frame structures with free viscoelastic layers: New closed-form solutions of eigenvalues and a viscous approach (submitted to Engineering Structures) 191

7.1 Introduction . . . . . . . . . . . . . . . . . . . . . . . 191

7.2 Finite element formulation in frequency domain. . . . . . . . . . . . . . . . . 194

7.2.1 Kinematic and constitutive relationships. . . . . . . . . . . . . . . 195

7.2 .2 Motion equation in frequency domain . . . . . . . . . . . . . . . . . 197 
7.3 The viscous approach . . . . . . . . . . . . . . . 200

7.3.1 Closed-form expressions for eigenvalues . . . . . . . . . . . . . . . . 201

7.3.2 Approximation of the transfer function . . . . . . . . . . . . . 205

7.4 Numerical examples . . . . . . . . . . . . . . . . . . . . . 206

7.4.1 Example 1: Cantilever beam . . . . . . . . . . . . . . . . . 206

7.4.2 Example 2: Frame structure . . . . . . . . . . . . . . . . . . . . . 212

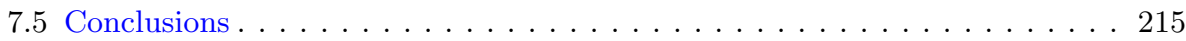

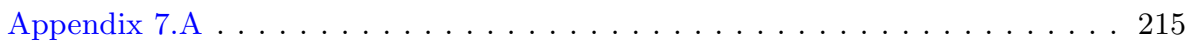

Bibliography . . . . . . . . . . . . . . . . . . . . . . . . 219

8 Conclusions 225

8.1 Summary . . . . . . . . . . . . . . . . . . . . . . . 225

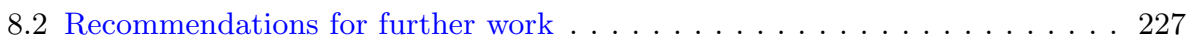





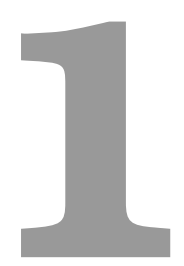

\section{Introduction}

"I think that it is a relatively good approximation to truth — which is much too complicated to allow anything but approximations - that mathematical ideas

originate in empirics. But, once they are conceived, the subject begins to live a peculiar life of its own and is governed by almost entirely aesthetical motivations"

John von Neumann, 1947

\subsection{Motivation and objectives}

The nature of the energy dissipation mechanisms in a vibrating structure has always been of very difficult explanation: the damping models have been developed trying to fit experimental and mathematical results. In this sense, the viscous approach by Rayleigh with dissipative forces proportional to the velocity of the system degrees of freedom (dof), has survived to today as the most used damping modeling for the great majority of structural solid materials (metals, concrete, wood, glass, masonry,...). The weakness of the pure viscous model is evident in application of the so-called viscoelastic materials, formed by polymer derivatives, rubbers and rubber-like materials and characterized by a time-dependent constitutive model and by frequency-dependent Young and shear moduli. In fact, these mechanical properties are usually called complex moduli since they present real and imaginary part. When these materials are part of a more complex struc- 
ture, the constitutive relationships lead to a frequency-dependent stiffness matrix. Viscoelastic materials have extensively been used for vibrating control in almost all areas of engineering: mechanical, architecture, civil, industrial, aeronautics and automotive. Among other applications, it must be mentioned the viscoelastic dampers or the viscoelastic layers (unconstrained or constrained) perfectly bonded on a base material. Although the term viscoelastic damping has traditionally been used, in the last years the concept non-viscous damping is also found in the bibliography, since this behavior can be considered as a generalization of the more basic viscous damping.

The first ideas of this thesis were born as consequence of the author's special interest on viscoelastic models for energy dissipation of vibrating systems and on the necessity to develop new numerical approaches for their analysis. The timeand frequency-dependence of the constitutive relationships and the application of the laws of dynamics lead to

1. In the time domain, a system of integro-differential equations.

2. In the frequency domain, a nonlinear eigenvalue problem for the solution of the free motion equations.

The work is focused on the second item, i.e. the analysis of the nonlinear eigenvalue problem and its applications. The main motivation has been the research of approximated solutions of the viscoelastic problem through the response of certain equivalent viscous model, the construction of which is in fact based on the eigenproblem. The principal objectives of the current thesis can be summarized in two items

O1 To propose new and efficient numerical methods to obtain the eigensolutions of the nonlinear eigenvalue problem of linear viscoelastic systems.

O2 To develop a new viscous model, called Equivalent Viscous Model, from the information provided by the previous eigensolutions and capable to accurately reproduce the response of the viscoelastic model under certain conditions.

A common purpose in each chapter has always been to give mathematical support to each obtained conclusion or result, although they intuitively could be applied without proof. Thus, rigorous convergence analysis or error-order studies are attached with every numerical algorithm. In this introduction the theoretical fundamentals of dynamic systems with viscoelastic damping are summarized, to later present a brief abstract of the key ideas related to the contributions made. 


\subsection{Dynamics of viscoelastic structures}

Let us consider an $N$ degrees-of-freedom (dof) vibrating system. The dofs' timedomain response is represented by a vector $\mathbf{u}(t) \in \mathbb{R}^{N}$. With help of the Finite Element Method the mass and the stiffness matrices of the system, denoted respectively by $\mathbf{M}, \mathbf{K} \in \mathbb{R}^{N \times N}$, can be assembled. In general, $\mathbf{M}, \mathbf{K}$ are symmetric and positive definite and semi-definite respectively. The viscoelastic damping is introduced in the system assuming that the dissipative forces $\mathbf{f}_{d}(t) \in \mathbb{R}^{N}$ are proportional to the history of the dofs' velocities via kernel hereditary functions. These functions are the entries of a matrix denoted by $\mathcal{G}(t) \in \mathbb{R}^{N \times N}$, also assumed symmetric.

$$
\mathfrak{f}_{d}(t)=\int_{-\infty}^{t} \mathcal{G}(t-\tau) \dot{\mathfrak{u}}(\tau) d \tau
$$

This representation of the hereditary behavior was originally introduced by Boltzman [1] at the end of XIX century. Its application to viscoelastic materials and to damping of vibrating systems was studied by different authors in the middle of the XX century. Among them, it is worth mentioning Gross [2-4], Volterra [5] and specially Biot [6-8] whose multi-exponential hereditary model given by

$$
\mathcal{G}(t)=\sum_{j=1}^{k} \mathbf{C}_{j} \mu_{j} e^{-\mu_{j} t}
$$

has widely been used for modeling viscoelastic damping materials. An interesting survey of the beginning and evolution of these models can be found in the paper of Bert [9]. The fundamentals of viscoelasticity, a thorough study on the time-dependence constitutive models and its application for modeling damping materials can be found in books such as Fluegge [10], Nashif [11] and Jones [12]. In the book of Sun [13] analytical solutions for different configurations are provided together with the implementation within finite element models. Other mathematical forms of $\mathcal{G}(t) \in \mathbb{R}^{N \times N}$ can be proposed, although they should be subjected to some restrictions to guarantee a strictly dissipative free motion; in mathematical terms the dynamic system defined by Eq. (1.3) is stable. Such conditions have been studied by Golla and Hughes in the reference [14], with a description of the constitutive viscoelastic models and their related implementation within the finite element method. Hence, a damping model characterized by the matrix $\mathcal{G}(t)$ or by its Laplace transform $\mathbf{G}(s)=\mathcal{L}\{\mathcal{G}(t)\}$ will lead to a strictly dissipative response if the following conditions are satisfied

1. $\mathcal{G}_{j k}(t)=\mathcal{G}_{k j}(t)$

2. $\mathcal{G}_{j k}(t)$ is decreasing in $t \geq 0$

3. $\lim _{t \rightarrow \infty} \mathcal{G}(t)=\mathbf{0}$ 
4. $\Re\{\mathbf{G}(i \omega)\}>0, \forall \omega>0$

After Biot's model other hereditary forms have been proposed, several of them cited in the work of Golla and Hughes. A more complete set including some recent proposals is listed in Table 2.1 of Chapter 2. Among these hereditary forms, the damping functions characteristic of the so-called fractional models must be emphasized. Such models assume constitutive relationships based on differential equations on fractional derivatives of stress and strain. The applications to structural dynamics is due to Bagley and Torvik [15-19], works that are considered pioneers in the field. The research of Pritz [20-25] on the four-parameter model and on the behavior of complex properties of rubbers and rubber-like materials has also been of great influence. A review on the application of fractional calculus to structural dynamics can be found in [26].

Assuming small displacements, the motion equations linearly relate the dynamic balance among inertia $\mathbf{M} \ddot{\mathfrak{u}}$, elastic $\mathbf{K} \mathfrak{u}$, damping $\mathfrak{f}_{d}(t)$, and external forces $\mathfrak{f}_{e}(t)$. The general form of the system of integro-diferential equations can be written by

$$
\begin{aligned}
& \mathbf{M} \ddot{\mathfrak{u}}+\int_{-\infty}^{t} \mathcal{G}(t-\tau) \dot{\mathfrak{u}}(\tau) d \tau+\mathbf{K} \mathfrak{u}=\mathfrak{f}_{e}(t) \\
& \dot{\mathfrak{u}}(0)=\mathbf{v}_{0}, \quad \mathfrak{u}(0)=\mathbf{u}_{0}
\end{aligned}
$$

Early research on this equation quickly led to the Ph.D. dissertation of Sondipon Adhikari [27] and particularly to his paper Dynamics of Non-viscously Damped Linear Systems, 2002 [28], also included in his thesis. In this work Adhikari constructs the exact solution of Eq. (1.3) from the information given by the nonlinear eigenproblem assuming that the damping matrix adopts the general form

$$
\mathbf{G}(s)=\sum_{j=1}^{r} \frac{P_{j}(s)}{Q_{j}(s)} \mathbf{K}_{j}
$$

where $P_{j}(s), Q_{j}(s)$ are $s$-polynomials defined so that the resulting rational expression is either of order $s^{-1}$ or, in the case of viscous damping, constant. Under these conditions the modes of the system can be obtained as the non-trivial solutions of the free-motion problem obtained considering $\mathfrak{f}_{e}(t)=\mathbf{v}_{0}=\mathbf{u}_{0}=\mathbf{0}$ in Eq. (1.3). Thus, checking functions of the form $\mathfrak{u}(t)=\mathbf{u} e^{\text {st }}$ we obtain

$$
\left[s^{2} \mathbf{M}+s \mathbf{G}(s)+\mathbf{K}\right] \mathbf{u} \equiv \mathbf{D}(s) \mathbf{u}=\mathbf{0}
$$

where $\mathbf{D}(s): \mathbb{C} \rightarrow \mathbb{C}^{N \times N}$ is the so called dynamic stiffness matrix. Eq. (1.5) is the the nonlinear eigenvalue problem of a viscoelastically damped vibration system. The eigenvalues are then the roots of the equation

$$
\operatorname{det}[\mathbf{D}(s)]=0
$$


Assuming that the elements of $\mathbf{G}(s)$ are rational functions in which the aforementioned characteristics hold, it is clear that Eq. (1.6) can always be reduced to a polynomial. The set of eigenvalues contains in general $2 N+q$ distinct values distributed as

$$
\left\{\lambda_{1}, \ldots, \lambda_{N}, \lambda_{1}^{*}, \ldots, \lambda_{N}^{*}, \sigma_{1}, \ldots, \sigma_{q}\right\}
$$

where $\lambda_{j}, \lambda_{j}^{*} \in \mathbb{C}, 1 \leq j \leq N$ are $N$ conjugate complex pairs, corresponding to the modes with oscillatory nature. The rest, $\sigma_{j} \in \mathbb{R}^{-} 1 \leq j \leq q$ are negative real numbers that represent overcritically dammped modes, called non-viscous since they are a feature of non-viscous systems. Associated to each eigenvalue there exists an eigenvector: we denote $\mathbf{u}_{j}, \mathbf{u}_{j}^{*} \in \mathbb{C}^{N}, 1 \leq j \leq N$ to the complex eigenvectors associated to $\lambda_{j}, \lambda_{j}^{*}$, and $\mathbf{a}_{j} \in \mathbb{R}^{N}, 1 \leq j \leq q$ to the eigenvector associated to the real eigenvalue $\sigma_{j}$.

In the analysis and search of solutions of the eigenproblem, the proportionality of an $N$-dof system, i.e. the modal decoupling capability, becomes of special importance. As known, the undamped problem obtained from $\mathcal{G}(t) \equiv \mathbf{0}$ can always be diagonalized in the modal space of matrices $\mathbf{M}$ and $\mathbf{K}$. Denoting by $\phi_{j} \in \mathbb{R}^{N}$ to the $j$ th mass-homogenized real mode (in column) and by $\omega_{j}$ to its associated natural frequency, the classical orthogonal relations can be written as

$$
\boldsymbol{\phi}_{j}^{T} \mathbf{M} \phi_{k}=\delta_{j k}, \quad \boldsymbol{\phi}_{j}^{T} \mathbf{K} \phi_{k}=\omega_{j}^{2} \delta_{j k}
$$

where $\delta_{j k}$ is the Kronecker delta. The modal matrix $\boldsymbol{\Phi}$ groups in columns the $N$ undamped modes and is used to change the dof's to the modal coordinates by $\mathbf{u}=\boldsymbol{\Phi} \mathbf{q}$. Hence, Eq. (1.5) can be expressed as

$$
\left[s^{2} \mathbf{I}_{N}+s \boldsymbol{\Gamma}(s)+\boldsymbol{\Lambda}\right] \mathbf{q}=\mathbf{0}
$$

where $\boldsymbol{\Lambda}=\boldsymbol{\Phi}^{T} \mathbf{K} \boldsymbol{\Phi}=\operatorname{diag}\left[\omega_{j}^{2}\right]$ is the diagonal squared natural frequency matrix, and $\boldsymbol{\Gamma}(s)=\boldsymbol{\Phi}^{T} \mathbf{G}(s) \boldsymbol{\Phi}$ the damping matrix in the modal space. In general, the latter is not diagonal although under certain conditions it could become so. The systems with this property are called proportional, since the necessary and sufficient conditions for the modal decoupling are directly related with proportional relationships between the damping matrix $\mathbf{G}(s)$ and the dynamic matrices $\mathbf{M}, \mathbf{K}$ [29]. In some cases of non-proportionality, the matrix $\boldsymbol{\Gamma}\left(\lambda_{j}\right)$ is diagonally dominant but not purely diagonal, fact which is equivalent to assume as true

$$
\sum_{\substack{l=1 \\ l \neq k}}^{N}\left|\Gamma_{k l}\left(\lambda_{j}\right)\right|<\left|\Gamma_{k k}\left(\lambda_{j}\right)\right|, \quad \forall 1 \leq k, j \leq N
$$

The system is then said to be lightly non-proportional. This property is commonly assumed in many problems related to non-viscous damping [28, 30-32], and allows 
to approximate the determinant as product of the terms of its main diagonal as

$$
\operatorname{det}\left[s^{2} \mathbf{I}_{N}+s \boldsymbol{\Gamma}(s)+\boldsymbol{\Lambda}\right] \approx \prod_{j=1}^{N}\left(s^{2}+s \Gamma_{j j}(s)+\omega_{j}^{2}\right)
$$

Hence, the set of eigenvalues can be obtained from the following $N$ decoupled equations

$$
D_{j}(s)=s^{2}+s \Gamma_{j j}(s)+\omega_{j}^{2}=0, \quad 1 \leq j \leq N
$$

With the availability of eigensolutions, the exact solution of the transfer function $\mathbf{H}(s)=\mathbf{D}^{-1}(s)$, defined as the inverse of the dynamic stiffness, can be obtained. According to the developments of Adhikari, for symmetric systems $\mathbf{H}(s)$ has the following expression

$$
\mathbf{H}(s)=\sum_{j=1}^{N}\left[\frac{\gamma_{j} \mathbf{u}_{j} \mathbf{u}_{j}^{T}}{s-\lambda_{j}}+\frac{\gamma_{j}^{*} \mathbf{u}_{j}^{*} \mathbf{u}_{j}^{*^{T}}}{s-\lambda_{j}^{*}}\right]+\sum_{j=1}^{q} \frac{\eta_{j} \mathbf{a}_{j} \mathbf{a}_{j}^{T}}{s-\sigma_{j}}
$$

where

$$
\gamma_{j}=\left(\mathbf{u}_{j}^{T} \frac{\partial \mathbf{D}\left(\lambda_{j}\right)}{\partial s} \mathbf{u}_{j}\right)^{-1}, 1 \leq j \leq N \quad, \quad \eta_{j}=\left(\mathbf{a}_{j}^{T} \frac{\partial \mathbf{D}\left(\sigma_{j}\right)}{\partial s} \mathbf{a}_{j}\right)^{-1}, 1 \leq j \leq q
$$

As shown in the two previous equations, the information provided by the modal space of the nonlinear eigenproblem becomes of great importance to construct the exact solution, the analytic expression of which is available in [28]. The challenge remains to find new efficient computational tools to solve the eigensolutions of Eq. (1.5).

Presented the fundamentals of the dynamics of viscoelastic systems, the key points of the contributions developed in the current thesis are described in the next section.

\subsection{Thesis organization}

In order to achieve the objectives of the thesis, six research papers have been written, constituting the main body of the document in six self-contained chapters, from Chapter 2 to Chapter 7 . Each one of them is therefore organized like a research article, including introduction, conclusions and bibliography. A brief summary with the key results of each paper is presented now. 


\subsubsection{A new recursive scheme to eigenvalues' computation}

In Chapter 2 a novel iterative method to solve the eigenvalue problem of proportional or lightly non-proportional viscoelastic systems is presented. This work has been published in the paper

Mario Lázaro, José L. Pérez Aparicio, Marcel Epstein. Computation of eigenvalues in proportionally damped viscoelastic structures based on the fixed-point iteration. Applied Mathematics and Computation, 2012, 219(8), pp 3511-3529 [33].

As known, the nonlinear eigenproblem under the assumption of proportionality can be reduced to the solution of $N$ nonlinear equations as in Eq. (1.12). Hence, it is reasonable to present the theoretical developments for a single dof viscoelastic oscillator characterized by mass $m$, elastic rigidity $k$ and hereditary viscoelastic function $\mathcal{G}(t)$ with Laplace transform $G(s)=\mathcal{L}\{\mathcal{G}(t)\}$. The eigenvalues are then the roots of the equation

$$
m s^{2}+s G(s)+k=0
$$

in general formed by a pair of conjugate complex numbers and several real eigenvalues (non-viscous) that depends on the number of hereditary exponential kernels in the damping function. The original idea of this paper is to manipulate Eq. (1.15) in order to express it as follows

$$
m s^{2}+s G(s)+k=m[s-X(s)][s-Y(s)]
$$

where $X(s), Y(s)$ are certain functions that depend on the eigenparameter $s$ only via the damping function $G(s)$. Immediately it is clear that any eigenvalue, either complex or real, is fixed point either of $X(s)$ or $Y(s)$. Therefore, some of the following recursive schemes can intuitively lead to a solution.

$$
x_{n}=X\left(x_{n-1}\right), \quad y_{n}=Y\left(y_{n-1}\right)
$$

The mathematical results show that both sequences converge when they start in a nonreal complex number, i.e. with no-null imaginary part. The sequence $\left\{x_{n}\right\}$ stays always in the complex upper half plane $(\Im\{z\}>0)$ and converges to the eigenvalue with positive imaginary part while the sequence $\left\{y_{n}\right\}$ behaves equally but in the complex lower half plane converging to the conjugate complex. The convergence conditions are rigorously studied, using for it results of the Fixed Point Theory beyond the Banach contraction principle. It is proved that the success of the method is almost ensured when the initial point verifies $\Im\left\{x_{0}\right\}, \Im\left\{y_{0}\right\} \neq 0$, in fact failure cases have not yet been found. It is additionally proved that the local convergence velocity is linear and directly depends on the level of damping and on the viscoelasticity, understanding the latter as the level of variation $\partial G(s) / \partial s$ evaluated at the eigenvalue. Systems lightly damped converge very quickly whereas 
systems strongly damped do much more slowly. The numerical simulation shows that the method converges even for overdamped systems, which only have real eigenvalues.

\subsubsection{The non-viscous set}

In Chapter 3 the real eigenvalues (also called non-viscous eigenvalues) of viscoelastic systems are studied. The work is currently under review

Mario Lázaro, José L. Pérez Aparicio. Characterization of Real Eigenvalues in Linear Viscoelastic Oscillators and the Non-viscous Set. Submitted to Journal of Applied Mechanics, transactions of the ASME, paper ID JAM12-1529, under review.

The main contribution is the proof of a new property of these eigenvalues: if $\sigma \in \mathbb{R}$ is a real eigenvalue of a viscoelastic oscillator with mass $m$, rigidity $k$ and damping function $G(s)$, then $|G(\sigma)| \geq 2 \sqrt{k m}$. This property can be considered a generalization of the inequality $c \geq c_{\mathrm{cr}}=2 \sqrt{\mathrm{km}}$, i.e. the necessary and sufficient condition for real eigenvalues in pure viscous oscillators. However, in viscoelastic systems if a real eigenvalue satisfies the above relation, this does not imply overdammping of the system. In fact, non-viscous eigenvalues coexist with an oscillatory state provided by the pair of conjugate complex eigenvalues. Since the mathematical form of $G(s)$ is a priori known, at least theoretically it is always possible to construct the following set within the real numbers.

$$
\mathcal{B}=\left\{s \in \mathbb{R}^{-}:|G(s)| \geq 2 \sqrt{k m}\right\}
$$

It can be assured that this set contains all real eigenvalues, and is named nonviscous set. This is so since the only real eigenvalues of non-overcritically damped viscoelastic systems are those corresponding to the non-viscous modes. It is demonstrated that $\mathcal{B}$ is union of $N$ closed intervals when the damping function obeys a Biot's model of $N$ exponential hereditary kernels. Exact solutions of the limits of such intervals are developed in the paper for the particular cases of $N=1$ and $N=2$ kernels. Approximated solutions are proposed for the general case of $N$ kernels; these solutions are valid for lightly and moderately damped systems. Finally, the information provided by the non-viscous set is used for the other important contribution of this paper: the proposal of a new analytical expression to approximate the non-viscous eigenvalues. The method is validated and compared with other one-step approximation methods available from the literature, one of them proposed in this thesis, in Chapter 4. 


\subsubsection{The parametric method: single dof systems}

In Chapter 4, the fundamentals of the parametric method to compute complex and real eigenvalues for single dof viscoelastic oscillators are presented. This research is currently under review

Mario Lázaro, José L. Pérez Aparicio. Parametric solutions of the eigenvalue problem for single-degree-of-freedom viscoelastic systems. Submitted to AIAA Journal, paper ID 2012-03-J051941, under review

The function $G(s)$ characterizes the damping of a single dof oscillator in the frequency domain. As mentioned, different mathematical forms (see Table 2.1) have been proposed, the expressions of which do not only depend on the eigenparameter $s$, but also on one or more damping parameters, generally obtained fitting experimental and model data of the complex modulus in frequency-domain. The central idea of this work is to assume as variable one of these damping parameters, so that the damping function can be considered as a two-variable function $G(s, \theta)$. Thus, the nonlinear equation for the eigenvalues can be written as

$$
m s^{2}+s G(s, \theta)+k=0
$$

whose solutions are not complex numbers but complex functions of the form $s=$ $\lambda(\theta): \mathbb{R} \rightarrow \mathbb{C}$. Let us assume that an explicit solution of Eq. (1.19), say $\lambda_{0}$, is known when the damping parameter is evaluated at certain initial point $\theta=\theta_{0}$. The difference $h=\left|\theta-\theta_{0}\right|$ can be considered as a numerical perturbation of the initial state, $\lambda_{0}=\lambda\left(\theta_{0}\right)$, so that an approximate expression of $\lambda(\theta)$ can be constructed by asymptotic expansion up to order $\mathcal{O}\left(h^{k+1}\right)$ as

$$
\lambda(\theta) \approx \lambda_{0}+\sum_{n=1}^{k} \frac{\lambda^{(n)}\left(\theta_{0}\right)}{n !}\left(\theta-\theta_{0}\right)^{n}
$$

In addition, it is demonstrated that the first derivative of the eigenvalue $\lambda^{\prime}=d \lambda / d \theta$ always can be written as

$$
\lambda^{\prime}=-\lambda \Psi(\lambda, \theta)
$$

where the mathematical form of $\Psi(s, \theta): \mathbb{C} \times \mathbb{R} \rightarrow \mathbb{C}$ is known. In the paper, the asymptotic expansion of Eq. (1.20) is combined with Eq. (1.21), obtaining a quasi-closed form for the eigenvalue

$$
\hat{\lambda}_{k}(\theta)=\lambda_{0} e^{-Z(\theta)}
$$

where, for the evaluation of $Z(\theta)$, a numerical integration by quadrature is necessary (hence the term quasi-closed form). Moreover, the contributions of this paper are complemented by a study of the order of approximation, obtaining $\left|\hat{\lambda}_{k}(\theta)-\lambda(\theta)\right| \leq \mathcal{O}\left(h^{k+2}\right)$. Consequently, the solution of the asymptotic expansion is improved one order. The numerical examples validate the theoretical results 
showing that effectively the error does not only depend on the distance $h=\left|\theta-\theta_{0}\right|$, but also on the level of damping and on the viscoelasticity induced by the viscoelastic model. Thus, light damping (combined in general with low viscoelasticity) leads to more accurate approximations. The analysis is completed with the calculation of closed-form expressions for the non-viscous eigenvalues by the asymptotic expansion.

\subsubsection{The parametric method: multiple dof systems}

The fundamentals of the parametric method presented in the previous section are generalized in Chapter 5 for non-proportional multiple dof systems with a multiparametric representation of the damping matrix. This research has been published in the paper

Mario Lázaro, José L. Pérez Aparicio. Multiparametric Computation of Eigenvalues for Linear Viscoelastic Structures. Computers \& Structures, 2013, 117, pp 67-81 [34].

Let us consider a $N$-dof viscoelastic system controlled by the dynamic matrices, $\mathbf{M}, \mathbf{K}$ and $\mathbf{G}(s, \boldsymbol{\theta})$. Now, the damping matrix is assumed to be a function $\mathbf{G}(s, \boldsymbol{\theta})$ : $\mathbb{C} \times \mathbb{R}^{p} \rightarrow \mathbb{C}^{N \times N}$ that depends on a parametric array or multiparametric vector $\boldsymbol{\theta} \in \mathbb{R}^{N}$. The nonlinear eigenproblem can be stated as

$$
\left[s^{2} \mathbf{M}+s \mathbf{G}(s, \boldsymbol{\theta})+\mathbf{K}\right] \mathbf{u} \equiv \mathbf{D}(s, \boldsymbol{\theta}) \mathbf{u}=\mathbf{0}
$$

equation that defines the eigenvalues and eigenvectors as multivariable functions $\lambda_{j}(\boldsymbol{\theta}): \mathbb{R}^{N} \rightarrow \mathbb{C}$ y $\mathbf{u}_{j}(\boldsymbol{\theta}): \mathbb{R}^{N} \rightarrow \mathbb{C}^{N}$. For the great majority of the viscoelastic models, a particular point in the parametric domain (also named initial point $\boldsymbol{\theta}_{0}$ ) can always be chosen so that the eigenproblem $\mathbf{D}\left(s, \boldsymbol{\theta}_{0}\right) \mathbf{u}=\mathbf{0}$ becomes linear or at least quadratic in $s$. For these problems, several and efficient numerical methods are available in the literature. Chosen the initial point with these characteristics, the first-order approximations are

$$
\begin{aligned}
& \lambda_{j}(\boldsymbol{\theta})=\lambda_{j}^{0}+\left(\boldsymbol{\theta}-\boldsymbol{\theta}_{0}\right)^{T} \boldsymbol{\nabla} \lambda_{j}^{0}+\mathcal{O}\left(h^{2}\right) \\
& \mathbf{u}_{j}(\boldsymbol{\theta})=\mathbf{u}_{j}\left(\boldsymbol{\theta}_{0}\right)+\sum_{r=1}^{p} \frac{\partial \mathbf{u}_{j}\left(\boldsymbol{\theta}_{0}\right)}{\partial \theta_{r}}\left(\theta_{r}-\theta_{r 0}\right)+\mathcal{O}\left(h^{2}\right)
\end{aligned}
$$

where

$$
\nabla \lambda_{j}=\left\{\frac{\partial \lambda_{j}}{\partial \theta_{1}}, \ldots, \frac{\partial \lambda_{j}}{\partial \theta_{p}}\right\}^{T}
$$

is the eigenvalue's gradient respect to the multiparametric vector and $h=\left\|\boldsymbol{\theta}-\boldsymbol{\theta}_{0}\right\|$ the perturbation measure in the parametric domain. With help of the eigensensitivity expression respect to the damping parameters, an improved solution with 
order of approximation $\mathcal{O}\left(h^{3}\right)$ as solution of certain differential equation can be constructed. The mathematical results predict that the accuracy of the method directly depends on the level of damping, on the viscoelasticity (as occurred in single dof systems) and, in addition, on the level of non-proportionality, i.e., how high the modal coupling is. Several numerical examples (for discrete and continuous structures) are presented in order to validate the theoretical foundations

\subsubsection{The Equivalent Viscous Model}

In Chapter 6 the Equivalent Viscous Model is presented as a new approach to approximately represent the response of a multiple dof viscoelastic system. The fundamentals and the theoretical developments are presented in

Mario Lázaro, José L. Pérez Aparicio, Marcel Epstein An Equivalent Viscous Model for Linear Viscoelastic System. Submitted to Mechanical Systems and Signal Processing, paper ID MSSP12-63, under review.

As shown, the transfer function of a viscoelastic system contains all information of the response in the frequency domain and a closed-form expression from the complete solution of the nonlinear eigenproblem can be constructed as

$$
\mathbf{H}(s)=\sum_{j=1}^{N}\left[\frac{\gamma_{j} \mathbf{u}_{j} \mathbf{u}_{j}^{T}}{s-\lambda_{j}}+\frac{\gamma_{j}^{*} \mathbf{u}_{j}^{*} \mathbf{u}_{j}^{*^{T}}}{s-\lambda_{j}^{*}}\right]+\sum_{j=1}^{p} \frac{\eta_{j} \mathbf{a}_{j} \mathbf{a}_{j}^{T}}{s-\sigma_{j}}
$$

where $\left\{\lambda_{j}, \mathbf{u}_{j}\right\} 1 \leq j \leq N$ are the complex eigenpairs and $\left\{\sigma_{j}, \mathbf{a}_{j}\right\} 1 \leq j \leq p$ the non-viscous eigenvalues. The rest of coefficients are explained in Eq. (1.14). The idea of developing a viscous model that reproduces the exact response, arises from the satisfactory numerical experiences carried out with the following transfer function

$$
\mathbf{H}_{e}(s)=\sum_{j=1}^{N} \frac{\phi_{j} \phi_{j}^{T}}{s^{2}+2 s \omega_{e j} \zeta_{e j}+\omega_{e j}^{2}}
$$

where the characteristics of the $j$ th mode, $\zeta_{e j}$ and $\omega_{e j}$, are expressed as function of the $j$ th complex pair as

$$
\zeta_{e j}=-\frac{\lambda_{j}+\lambda_{j}^{*}}{2 \sqrt{\lambda_{j} \lambda_{j}^{*}}}, \quad \omega_{e j}=\sqrt{\lambda_{j} \lambda_{j}^{*}}
$$

Notice that Eq. (1.27) is a transfer function of a viscous system characterized by certain specially developed dynamic matrices $\mathbf{M}_{e}, \mathbf{C}_{e}$ and $\mathbf{K}_{e}$. These matrices can be constructed from the complex eigenvalues $\left\{\lambda_{j}\right\}$ and from the real modes $\left\{\phi_{j}\right\}$ of the undamped problem. The empirical experiences observed by the numerical simulations shown that the level of accuracy depends on two factors. On one hand 
the non-proportionality of the system, measured through an index denoted by $\alpha \geq 0 ; \alpha=0$ represents total decoupling and proportional damping. On the other hand, how strong the damping induced by the model $\mathbf{G}(s)$ is and how high is the viscoelasticity or, equivalently, the level of variation $\partial \mathbf{G}(s) / \partial s$. Both phenomenons are quantified by the index $\beta \geq 0$, so that $\beta=0$ represents the undamped state. The main contribution of this paper is to demonstrate that effectively the exact transfer function can be expressed as

$$
\mathbf{H}(s)=\mathbf{H}_{e}(s)+\delta \mathbf{H}(s)=\mathbf{H}_{e}(s)+\mathcal{O}(\alpha, \beta)
$$

The accuracy of the equivalent viscous model is evaluated through several numerical examples, using discrete systems with different levels of damping and proportionality. This method is very useful in the analysis of structures with light non-proportionality for for which an efficient form for computing the complex eigenvalues is available. Such case is described in Chapter 7, summarized in the following section.

\subsubsection{An application to frame structures with free viscoelastic layers}

In Chapter 7 the equivalent viscous model is applied to frame structures damped with free viscoelastic layers. In addition, a new variant of the parametric method is proposed deriving new closed-form expressions for the complex eigenvalues. These contributions have been developed in the paper

Mario Lázaro, José L. Pérez Aparicio. Dynamic analysis of frame structures with free viscoelastic layers: new closed-form solutions of eigenvalues and a viscous approach. Submitted to Engineering Structures,Structures, paper ENGSTRUCT-D-12-01352, under review

The studied structures are frames formed by one dimensional beams with bonded unconstrained viscoelastic damping layers. The layers are perfectly bonded on the top and/or on the bottom of the base material, in general metallic. With this layout the damping material works under tension-compression mechanisms. The constitutive relationships between stress $\sigma_{x}$ and strain $\epsilon_{x}$ for the damping material is based on the fractional derivatives.

$$
\sigma_{x}+\tau^{\alpha} \frac{\mathrm{d}^{\alpha} \sigma_{x}}{\mathrm{~d} t^{\alpha}}=E_{0}\left(\epsilon_{x}+c \tau^{\alpha} \frac{\mathrm{d}^{\alpha} \epsilon_{x}}{\mathrm{~d} t^{\alpha}}\right)
$$

where $E_{0}$ is the static Young modulus and $\alpha, c, \tau$ the parameters that control the dissipative mechanisms. The above expression can be expressed in the Laplace domain as

$$
\hat{\sigma}_{x}(s)=E_{0} \frac{1+c(s \tau)^{\alpha}}{1+(s \tau)^{\alpha}} \hat{\epsilon}_{x}(s) \equiv \hat{E}_{v}(s) \hat{\epsilon}_{x}(s)
$$


With the help of the finite element method and the virtual work principle the motion equation in the frequency domain are constructued. Although the damping model has three parameters, only the storage coefficient $c$ is assumed to be variable. According to the parametric method the eigenvalues, considered now functions of the form $\lambda_{j}(c)$, are solutions of certain differential equation. Since the variation of these eigenvalues with the storage coefficient is specially smooth, the developments lead in this case to closed-form expressions. Consequently, no computational effort is required for their calculation except the one needed for the undamped linear eigenproblem solution. Furthermore, closed-forms of the new viscous damping and stiffness matrices, $\mathbf{C}_{e}$ and $\mathbf{K}_{e}$, are specially developed. Again, the accuracy of the proposed method is checked and validated by two numerical examples, for that different materials under several levels of damping. 



\section{Bibliography}

[1] Ludwig Boltzmann. "Zur Theorie der elastischen Nachwirkung." In: Annalen der Physik 241.11 (1878), 430432 (cit. on p. 3).

[2] B. Gross. "On creep and relaxation." In: Journal of Applied Physics 18.2 (1947), pp. 212-221 (cit. on p. 3).

[3] B. Gross. "On creep and relaxation. II." In: Journal of Applied Physics 19.3 (1948), pp. 257-264 (cit. on p. 3).

[4] B.a Gross and H.b Pelzer. "On creep and relaxation. III." In: Journal of Applied Physics 22.8 (1951), pp. 1035-1039 (cit. on p. 3).

[5] E. Volterra. "Vibrations of elastic systems having hereditary characteristic." In: Journal of Applied Mechanics 17 (1950), pp. 363-371 (cit. on p. 3).

[6] M.A. Biot. "Theory of Stress-Strain Relations in Anisotropic Viscoelasticity and Relaxation Phenomena." In: Journal Of Applied Physics 25.11 (1954), 1385-1391 (cit. on p. 3).

[7] M.A. Biot. "Variational Principles in Irreversible Thermodynamics with Application to Viscoelasticity." In: Physical Review 97.6 (1955), 1463-1469 (cit. on pp. 3, 20, 24, 37, 58, 82, 93, 192).

[8] M.A. Biot. "Linear thermodynamics and the mechanics of solids." In: Proc. Third U. S. National Congress on Applied Mechanics, ASME, New York. 1958 (cit. on p. 3).

[9] C.W. Bert. "Material Damping - Introductory Review Of Mathematicalmodels, Measures And Experimental Techniques." In: Journal of Sound and Vibration 29.2 (1973), 129-153 (cit. on p. 3).

[10] W. Flugge. Viscoelasticity. 2nd Edition. Springer-Verlag, 1975 (cit. on pp. 3, 160).

[11] A.D. Nashif, D.I.G. Jones, and J.P. Henderson. Vibration Damping. John Wiley and Sons, 1985 (cit. on pp. 3, 45, 160, 192, 194, 206).

[12] David I.G. Jones. Handbook of viscoelastic vibration damping. John Wiley \& Sons, 2001 (cit. on pp. 3, 45, 160, 192, 194). 
[13] C.T. Sun and Y.P. Lu. Vibration Damping of Structural Elements. Prentice Hall PTR, 1995 (cit. on pp. 3, 192, 194).

[14] D.F. Golla and P.C. Hughes. "Dynamics of Viscoelastic Structures - A Time-domain, Finite-element Formulation." In: Journal of Applied MechanicsTransactions of the ASME 52.4 (1985), 897-906 (cit. on pp. 3, 20, 22, 24, $58,60,82,84,118,160,162,164,192)$.

[15] R.L. Bagley and P.J. Torvik. "A Theoretical Basis for the Application of Fractional Calculus to Viscoelasticity." In: Journal of Rheology 27.3 (1983), 201-210 (cit. on pp. 4, 20, 82, 192, 196).

[16] R.L. Bagley and P.J. Torvik. "Fractional Calculus - A Different Approach to the Analysis of Viscoelastically Damped Structures." In: AIAA Journal 21.5 (1983), 741-748 (cit. on pp. 4, 20, 24, 82, 192, 193, 196).

[17] R.L. Bagley and P.J. Torvik. "On the Appearance of the Fractional Derivative in the Behavior of Real Materials." In: Journal of Applied Mechanics 51.2 (1984), 294-298 (cit. on p. 4).

[18] R.L. Bagley and P.J. Torvik. "Fractional Calculus in the Transient Analysis of Viscoelastically Damped Structures." In: AIAA journal 23.6 (1985), 918 925 (cit. on pp. 4, 193).

[19] R.L. Bagley and P.J. Torvik. "On the Fractional Calculus Model of Viscoelastic Behavior." In: Journal of Rheology 30.1 (1986), 133-155 (cit. on pp. 4, 196, 206).

[20] T. Pritz. "Analysis of four-parameter fractional derivative model of real solid materials." In: Journal of Sound and Vibration 195.1 (1996), 103-115 (cit. on pp. 4, 42, 45, 150, 192, 196, 206).

[21] T. Pritz. "Frequency dependences of complex moduli and complex Posson's ratio of real solid materials." In: Journal of Sound and Vibration 214.1 (1998), 83-104 (cit. on pp. 4, 45, 150, 206).

[22] T Pritz. "Loss factor peak of viscoelastic materials: Magnitude to width relations." In: Journal of Sound and Vibration 246.2 (2001), 265-280 (cit. on pp. 4, 45, 150, 196, 206).

[23] T Pritz. "Five-parameter fractional derivative model for polymeric damping materials." In: Journal of Sound and Vibration 265.5 (2003), 935-952 (cit. on pp. 4,192$)$.

[24] T. Pritz. "The Poisson's loss factor of solid viscoelastic materials." In: Journal of Sound and Vibration 306.3-5 (2007), 790-802 (cit. on pp. 4, 206).

[25] T. Pritz. "Relation of bulk to shear loss factor of solid viscoelastic materials." In: Journal of Sound and Vibration 324.3-5 (2009), 514-519 (cit. on pp. 4, 206). 
[26] Yuriy A. Rossikhin and Marina V. Shitikova. "Application of Fractional Calculus for Dynamic Problems of Solid Mechanics: Novel Trends and Recent Results." In: Applied Mechanics Reviews 63.1 (2010), 010801(1)010801(52) (cit. on pp. 4, 20).

[27] S. Adhikari. "Damping Models for Structural Vibration." PhD thesis. Cambridge University Engineering Department, 2000 (cit. on p. 4).

[28] S. Adhikari. "Dynamics of Non-viscously Damped Linear Systems." In: Journal of Engineering Mechanics 128.3 (2002), 328-339 (cit. on pp. 4-6, $22,35,36,58,84,94,121,125,129,131,160-162,165,168,170,171,203)$.

[29] S. Adhikari. "Classical normal modes in non-viscously damped linear systems." In: AIAA Journal 39.5 (2001), pp. 978-980 (cit. on pp. 5, 35, 44, $63,84,121)$.

[30] J. Woodhouse. "Linear Damping Models For Structural Vibration." In: Journal of Sound and Vibration 215.3 (1998), 547-569 (cit. on pp. 5, 35, 58, 121, 170, 171).

[31] Sondipon Adhikari and Blanca Pascual. "Eigenvalues of linear viscoelastic systems." In: Journal of Sound and Vibration 325.4-5 (2009), 1000-1011 (cit. on pp. 5, 21, 35, 41, 42, 69, 70, 76, 83, 119, 120, 141, 151, 152, 193).

[32] Sondipon Adhikari and Blanca Pascual. "Iterative Methods for Eigenvalues of Viscoelastic Systems." In: Journal of Vibration and Acoustics 133.2 (2011), pp. 021002.1-021002.7 (cit. on pp. 5, 21, 35, 42, 59, 83, 108, 119, $171,193)$.

[33] M. Lázaro, J. L. Pérez-Aparicio, and M. Epstein. "Computation of eigenvalues in proportionally damped viscoelastic structures based on the fixedpoint iteration." In: Applied Mathematics and Computation 219.8 (2012), 3511-3529 (cit. on pp. 7, 59, 119, 193).

[34] M. Lázaro and J. L. Pérez-Aparicio. "Multiparametric computation of eigenvalues for linear viscoelastic structures." In: Computers \& Structures 117.0 (2013), pp. $67-81$ (cit. on pp. 10, 21, 194, 201). 



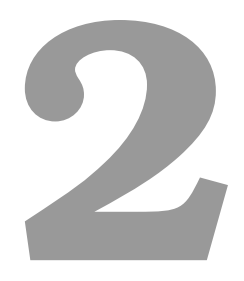

\section{Computation of eigenvalues in proportionally damped viscoelastic structures based on the fixed-point iteration}

Applied Mathematics and Computation, 219-8 (2012), 3511-3529

\subsection{Introduction}

Materials of viscoelastic nature are widely used for engineering applications such as vibration isolation or as devices to mitigate earthquake effects in buildings. In order to predict the behavior of such structures, the models must reproduce the response as accurately as possible. In the most general case, the structures that include viscoelastic materials are characterized by hereditary energy dissipation mechanisms: the damping forces depend on the history of the velocity response. Mathematically, this fact is represented by convolution integrals that involve the velocities of the degrees-of-freedom (dof) over certain kernel functions. In general, the dof response $\mathbf{u}(t) \in \mathbb{R}^{q}$ is governed by the following system of linear integro- 
differential equations

$$
\mathbf{M} \ddot{\mathbf{u}}+\int_{-\infty}^{t} \mathcal{G}(t-\tau) \dot{\mathbf{u}} \mathrm{d} \tau+\mathbf{K u}=\mathbf{F}(t)
$$

where $\mathbf{M} \in \mathbb{R}^{q \times q}$ and $\mathbf{K} \in \mathbb{R}^{q \times q}$ are the mass and stiffness matrices assembled using the finite element method. We assume $\mathbf{M}$ to be positive definite and $\mathbf{K}$ positive semidefinite; $\mathcal{G}(t) \in \mathbb{R}^{q \times q}$ is the viscoelastic damping matrix in the time domain.

Checking solutions with form $\mathbf{u}(t)=\mathbf{u}_{0} e^{s t}$ in the free-motion equation (2.1) with $\mathbf{F}(t) \equiv \mathbf{0}$, the following nonlinear eigenvalue problem is obtained

$$
\left[s^{2} \mathbf{M}+s \mathbf{G}(s)+\mathbf{K}\right] \mathbf{u}_{0}=\mathbf{0}
$$

where $\boldsymbol{G}(s)=\mathcal{L}\{\mathcal{G}(t)\}$ is the damping matrix in the Laplace domain. Many real structures modeled by Eq. (2.1) present a proportional - or lightly nonproportional- damping matrix, that is, $\mathbf{G}(s)$ becomes diagonal - or diagonallydominant - in the modal space of the undamped problem. The diagonalization greatly simplifies the calculation of the eigenvalues, since the characteristic equation can be approximated as a product of $q$ decoupled modal equations. The knowledge of the eigensolutions of the previous problem is a key issue in the analysis and study of viscoelastic structures, hence the importance of the development of efficient tools oriented to their numerical computation.

The seminal work of Biot [7] was a starting point in the variational justification of hereditary constitutive models based on exponential decay. Subsequently, experimental results led to extend these models with the introduction of the fractional derivative as a highly effective tool. Papers of Bagley and Torvik [15, 16] established the theoretical basis for the application of fractional derivatives in structural dynamics. Since then, a great number of papers have studied the resulting differential equations in fractional derivatives; among others [26] the works of Ray et al. [35-37] are of special interest. Both Biot's and fractional derivative models are associated with a different damping function $\mathcal{G}(t)$. The conditions under which this function defines a strictly dissipative motion were studied by Golla and Hughes [14]. Thus, new models could be introduced provided that they satisfy the requirements from [14]. For instance, Buhariwala [38, 39] generalized Biot's model with another in which the relaxation parameters are distributed in an interval. Of special interest are models based on the state-space approach such as the GHM approach of Golla, Hughes and McTavish [14, 40] or the Anelastic Displacement Field of Lesieutre and Mingori [41]. Both of them are characterized by the introduction of new internal variables. Adhikari and Woodhouse [42, 43] proposed new viscoelastic functions in the context of viscous and non-viscous damping identification for dynamic systems. These viscoelastic functions are the most used in 
the field of structural dynamics and all of them are strictly decreasing in the time domain. However, kernels not necessarily decreasing can also be compatible with viscoelastic problems as it has been demonstrated in the works of Medjden and Tatar [44] and Tatar [45].

Since the linearity is preserved in viscoelastic structures, it is logical to consider the associated eigenvalue problem. The main difference between the models of these systems and those of viscous ones is that for the former the damping matrix is frequency-dependent, see Eq (2.2), and that the eigenvalue problem is nonlinear. Among the numerical methods oriented to solve this problem, we mention first the works of Yang [46] and Singh [47], developed for any form of the transcendental matrix — named dynamic stiffness matrix in our context-. Both of them are based on the widely used Newton-Raphson method; although the iterative scheme is locally convergent with quadratic speed, the effectiveness depends on the chosen starting point. Another method from Williams and Kennedy [48] suggested a parabolic interpolation of the determinant of the dynamic stiffness matrix. Asymptotic techniques based on perturbation of the damping matrix were proposed by Daya and Potier-Ferry [49] and by Duigou et al. [50]. Voss [51, 52] introduced two methods based on the Arnoldi's shift-and-invert technique and on the Jacobi-Davidson method, respectively. Abdel-Aziz and El-Sayed [53] studied the sensitivity analysis of the eigenvalue functions of non-linear eigensystems under the assumption that such functions are continuous nondifferentiable. Specific methods aimed at solving the problem when the damping function is rational can be found. For instance, Muravyov and Menon [54-56] developed approaches that essentially transform the nonlinear problem into a larger linear one. Although their solution is exact, the numerical complexity increases with the polynomial degree of the damping function denominator. For proportional or lightly non-proportional systems, Adhikari and Pascual [31, 32] proposed several iterative methods based on Taylor series expansion of the damping function to compute complex and real eigenvalues. Friswell and Adhikari [57] proposed a new non-local viscoelastic foundation model for beams and obtained the eigensolutions using the finite element method. The introduction of eigenvalues' derivatives in viscoelastic structures [58, 59] has allowed the development of new numerical methods for the computation of the eigensolutions. Cortés and Elejabarrieta [60,61] developed an approach using the eigenvalue sensitivities, even applicable for highly damped systems. MartinezAguirre and Elejabarrieta [62] used higher order eigensensitivities to propose a new numerical procedure for the modal analysis of viscoelastic damped structures. Lázaro and Pérez-Aparicio [34, 63] have carried out recently new proposals based on considering a parametric treatment of the eigensolutions; in them the eigenvalue problem is transformed into an ordinary differential equation. 
The present paper develops a new numerical method to compute the eigenvalues of linear viscoelastic structures with proportional — or lightly non-proportionaldamping. The key idea is to build two complex-valued functions of a complex variable, whose fixed points are the eigenvalues. These functions uniquely depend on the damping and do not require the calculation of derivatives for their construction. It is shown that the use of the fixed-point iteration always allows to find the complex eigenvalues; moreover, it is demonstrated that the level of damping is directly related to the speed of convergence. Under certain conditions the method can also be applied to the computation of the non-viscous real eigenvalues. Finally, theoretical results are illustrated with two numerical examples. First, a single dof system with an exponential damping model is analyzed; since the overdamped region of this system can be analytically defined, this example allows to relate the level of damping and the speed of convergence. Second, a four-dof system with viscoelastic links is analyzed to validate the method for multiple dof systems with proportional damping.

\subsection{Single Degree--of-Freedom Systems}

\subsubsection{Eigenvalue Problem and Recursive Functions}

The theoretical fundamentals of the proposed method are developed for single degree-of-freedom systems (sdof). As mentioned, for non-viscously damped systems the dissipative forces are history-dependent on the velocity of the dof $\dot{u}(t)$ via a kernel function $\mathcal{G}(t)$, a characteristic of the damping model. The sdof free motion equation is the counterpart of Eq. (2.1)

$$
m \ddot{u}+\int_{-\infty}^{t} \mathcal{G}(t-\tau) \dot{u} \mathrm{~d} \tau+k u=0
$$

where $m$ and $k$ are the mass and the linear stiffness associated with the sdof, respectively. Checking solutions with the form $u(t)=u_{0} e^{s t}$, the previous equation is transformed into the following in terms of the variable $s$

$$
m s^{2}+s G(s)+k=0
$$

where again $G(s)=\mathcal{L}\{\mathcal{G}(t)\}$ is the viscoelastic damping function. Golla and Hughes [14] gave the necessary conditions on $G(s)$ to define a strictly dissipative viscoelastic behavior. Several authors $[28,64]$ have shown that the characteristic Eq. (2.4) has $2+p(p \geq 0)$ eigenvalues with form $\left\{\lambda, \lambda^{*}, \sigma_{1}, \ldots, \sigma_{p}\right\}$. The roots $\lambda, \lambda^{*}$ are a pair of complex-conjugate numbers associated with exponential-decay oscillatory modes. The rest $\sigma_{r}, 1 \leq r \leq p$, are negative real numbers named nonoscillatory or non-viscous eigenvalues since they are associated with overcritically damped modes, [28]. 
The eigenvalues of the undamped problem $G(s) \equiv 0$ are $\pm i \omega_{u}$, where $\omega_{u}=\sqrt{k / m}$ is the undamped natural frequency. Introducing the new non-dimensional function

$$
J(s)=\frac{G(s)}{2 m \omega_{u}}
$$

and using $k=m \omega_{u}^{2}$, Eq. (2.4) can be written as

$$
s^{2}+2 \omega_{u} s J(s)+\omega_{u}^{2}=0
$$

The key issue of the method is to transform this equation so that the resulting structure permits to apply a recursive scheme. For that, let us assume that Eq. (2.6) may be expressed in the form

$$
s^{2}+2 \omega_{u} s J(s)+\omega_{u}^{2} \equiv[s+A(s)]^{2}+B(s)
$$

There exist infinite solutions for the unknown functions $A(s), B(s)$; in particular one pair can be obtained identifying the coefficients of the terms $s^{0}=1, s^{1}=s$. Thus,

$$
\begin{aligned}
& A(s)=\omega_{u} J(s) \\
& B(s)=\omega_{u}^{2}\left[1-J^{2}(s)\right]
\end{aligned}
$$

Notice that the solutions given in Eq. (2.8) are not unique, and as will be shown, this proposed solution has important properties. Using the new functions, further changes can be achieved in Eq. (2.7) to obtain a more suitable expression. Eq. (2.7) together Eq. (2.8) produces a difference of two squares, resulting in

$$
\begin{aligned}
s^{2}+2 \omega_{u} s J(s)+\omega_{u}^{2} & =\left[s+\omega_{u} J(s)\right]^{2}+\omega_{u}^{2}\left[1-J^{2}(s)\right] \\
& =\left[s+\omega_{u} J(s)\right]^{2}-\left(i \omega_{u} \sqrt{1-J^{2}(s)}\right)^{2} \\
& =[s-X(s)][s-Y(s)]
\end{aligned}
$$

where $i=\sqrt{-1}$ is the imaginary unity and the complex functions $X(s), Y(s)$ are defined as

$$
X(s)=\omega_{u}\left(-J(s)+i \sqrt{1-J^{2}(s)}\right), \quad Y(s)=\omega_{u}\left(-J(s)-i \sqrt{1-J^{2}(s)}\right)
$$

In the previous definition, $\sqrt{\bullet}$ must be understood as the principal square root of a complex number. Eq. (2.9) states that, if $a \in \mathbb{C}$ represents any eigenvalue of the viscoelastic system, then either $a=X(a)$ or $a=Y(a)$, that is, $a$ is a fixed point of some of the two functions $X(s)$ or $Y(s)$. The objective is now to provide the conditions under which these functions may be used in a recursive scheme to calculate the eigenvalues. Previously, some definitions are given to present the hypotheses assumed for the damping function $J(s)$. Let us define the sets

$$
\mathcal{C}_{+}=\{x+i y \in \mathbb{C}: y>0\}, \quad \mathcal{C}_{-}=\{x+i y \in \mathbb{C}: y<0\}
$$


The complex plane excluding real numbers is named $\mathcal{C}=\mathcal{C}_{+} \cup \mathcal{C}_{-}=\mathbb{C} \backslash \mathbb{R}$.

As mentioned, we assume that $G(s)=2 m \omega_{u} J(s)$ verifies the required conditions given by [14] to induce a dissipative motion. In addition, the following two hypotheses are required for the purposes of the current work

H1. $G(s)$ is analytical in a domain with form $\mathbb{C} \backslash \mathcal{M}$, where $\mathcal{M} \subset \mathbb{R}$ is a subset of real numbers

H2. $G(s) \in \mathcal{C}, \forall s \in \mathcal{C}$. Expressed in set operations: $G(\mathcal{C}) \subset \mathcal{C}$

The set $\mathcal{M}$ introduced in H1 may adopt different forms depending on the type of damping function considered. Table 2.1 shows the most used damping functions from the bibliography and their associated sets $\mathcal{M}$. It can be observed that, although $\mathrm{H} 1$ assumes the most general case $\mathcal{M} \subset \mathbb{R}$, for real systems $\mathcal{M} \subset \mathbb{R}^{-} \cup\{0\}$ in order to guarantee the energy decay during the free motion.

\begin{tabular}{|c|c|c|}
\hline Damping functions, $G(s)$ & Set $\mathcal{M}$ & Author, Year, Reference \\
\hline$\sum_{k=1}^{n} \frac{a_{k}}{s+b_{k}}, \quad b_{k}>0$ & $\left\{-b_{k}\right\}_{k=1}^{n}$ & Biot, $1955[7]$ \\
\hline$\frac{c}{b-a} \log \frac{s+b}{s+a}, \quad 0<a<b$ & {$[-b,-a]$} & Buhariwala, $1982[38,39]$ \\
\hline$\frac{1}{s} \frac{E_{1} s^{\alpha}-E_{0} b s^{\beta}}{1+b s^{\beta}}, \quad 0<\alpha, \beta<1$ & $\mathbb{R}^{-} \cup\{0\}$ & Bagley \& Torvik, 1983 [16] \\
\hline$\frac{G^{\infty}}{s}\left[1+\sum_{k=1}^{n} \alpha_{k} \frac{s^{2}+2 \zeta_{k} \omega_{k} s}{s^{2}+2 \zeta_{k} \omega_{k} s+\omega_{k}^{2}}\right]$ & $\begin{array}{l}\left\{-p_{k},-q_{k}\right\}_{k=1}^{n} \cup\{0\} \\
p_{k}, q_{k}=-\omega_{k}\left(\zeta_{k} \pm \sqrt{\zeta_{k}^{2}-1}\right)\end{array}$ & $\begin{array}{l}\text { Golla \& Hughes, } 1983 \text { [14] } \\
\text { McTavish \& Hughes, } 1993 \text { [40] }\end{array}$ \\
\hline$\frac{1}{s}\left[1+\sum_{k=1}^{n} \frac{\Delta_{k} s}{s+\beta_{k}}\right], \quad \beta_{k}>0$ & $\left\{-\beta_{k}\right\}_{k=1}^{n} \cup\{0\}$ & Lesieutre \& Mingori, 1990 [41] \\
\hline$c \frac{1-e^{-s t_{0}}}{s t_{0}}$ & $\emptyset$ & Adhikari, 1998 [42] \\
\hline$c e^{s^{2} / 4 \mu}\left[1-\operatorname{erf}\left(\frac{s}{2 \sqrt{\mu}}\right)\right]$ & $\emptyset$ & Adhikari \& Woodhouse, 2001 [43] \\
\hline
\end{tabular}

Table 2.1: Damping functions from the bibliography and their corresponding sets $\mathcal{M} \subset \mathbb{R}$

The functions $X(s), Y(s)$ introduced in Eqs. (2.10) are directly related with the dimensionless damping function $J(s)=G(s) / 2 m \omega_{u}$. In order to define mathematically the region where $X(s)$ and $Y(s)$ are analytical, let us introduce the set

$$
\mathcal{B}=\{x \in \mathbb{R}:|J(x)| \geq 1\}
$$


Assuming that $G(s)$ satisfies $\mathrm{H} 1, \mathrm{H} 2$, the following properties for $X(s), Y(s)$ can be established (see 2.5 for the proof details)

P1. The functions $X(s), Y(s)$ are analytic in the set $\mathcal{A}=\mathbb{C} \backslash(\mathcal{M} \cup \mathcal{B})$

P2. $X^{*}(s)=Y\left(s^{*}\right), \forall s \in \mathcal{A}$

P3. Let $a \in \mathcal{A}$ be a complex number. Then, $a=X(a)$ if and only if $a^{*}=Y\left(a^{*}\right)$

P4. $X(\mathcal{C}) \subset \mathcal{C}_{+}, \quad Y(\mathcal{C}) \subset \mathcal{C}_{-}$

P5. If $J(\mathbb{R}) \subset \mathbb{R}$ then $X(\mathcal{B}) \subset \mathbb{R}, X(\mathbb{R} \backslash \mathcal{B}) \subset \mathcal{C}_{+}$and also $Y(\mathcal{B}) \subset \mathbb{R}$, $Y(\mathbb{R} \backslash \mathcal{B}) \subset \mathcal{C}_{-}$

The five properties constitute the starting point to analyze the numerical solution of Eq. (2.4) from a recursive point of view.

\subsubsection{Complex Eigenvalues}

The properties P1-P5 allow to state that, if $\lambda \in \mathcal{C}_{+}$and $\lambda^{*} \in \mathcal{C}_{-}$are the complex conjugate pair solution of Eq. (2.4), then $\lambda=X(\lambda), \lambda^{*}=Y\left(\lambda^{*}\right)$. In other words, $\lambda, \lambda^{*}$ are fixed points of functions $X$ and $Y$, respectively. Let us consider two any complex numbers $x_{0} \in \mathcal{C}_{+}, y_{0} \in \mathcal{C}_{-}$and let $\left\{x_{n}\right\},\left\{y_{n}\right\}$ be the recursive sequences of iterates defined for $n \geq 1$ as

$$
x_{n}=X\left(x_{n-1}\right), \quad y_{n}=Y\left(y_{n-1}\right)
$$

The question is whether these sequences converge or not to fixed points, and therefore, to solutions of the nonlinear eigenvalue problem. An affirmative answer to this question would allow us to use Eqs. (2.13) as an efficient numerical tool to compute the complex eigenvalues. This subsection is aimed to find answers to the following questions:

- Under what conditions the existence of complex fixed points of $X(s), Y(s)$ can be ensured?

- Can a recursive scheme be constructed so that it converges to these fixed points, regardless of the starting point?

- What is the speed of convergence of the recursive scheme?

The problem of the existence of fixed points will be solved using a known result in Fixed Point Theory due to Goebel, Sekowski and Stachura [65]. For that, it is necessary to define the concept of strict inclusion in sets. 
Definition 1 (Strict inclusion). Let $S, T \subset \mathbb{C}$ two complex sets; $S$ is said to lie strictly inside $T$ if there exists a real positive number $\epsilon>0$ such that $B(w, \epsilon) \subset T$, $\forall w \in S$. Here, $B(w, \epsilon)$ is the closed ball centered at $w$ with radius $\epsilon$

Lemma 1 (Goebel, Sekowski and Stachura [65]). Let $h: \mathbb{D} \rightarrow \mathbb{D}$ be an analytic self-mapping defined in the open unit ball $\mathbb{D}=\{w \in \mathbb{C}:|w|<1\}$. Let $w_{n}=$ $h\left(w_{n-1}\right)$ be the sequence of iterates of function $h(w)$. Then $h$ has a fixed point in $\mathbb{D}$ if and only if there exists a point $w_{0} \in \mathbb{D}$ such that strictly $\left\{w_{n}\right\}_{n \geq 1} \subset \mathbb{D}$

In addition, to answer the second question another result in Fixed Point Theory due to Reich [66] will be applied. This result is focused on the approximation to fixed points of holomorphic functions in the complex unit ball.

Lemma 2 (Reich [66]). Let $h: \mathbb{D} \rightarrow \mathbb{D}$ be an analytic self-mapping with a fixed point, and let $u_{0} \in \mathbb{D}$. Then the sequence $\left\{u_{n}\right\}$ where $0<\theta<1$

$$
u_{n}=\frac{u_{0}}{n^{\theta}}+\left(1-\frac{1}{n^{\theta}}\right) h\left(u_{n-1}\right), \quad n \geq 1
$$

converges strongly to a fixed point of $h(w)$.

Based on these results Theorem 1 states that, under relatively weak conditions, $X(s), Y(s)$ have fixed points in $\mathcal{C}_{+}$and $\mathcal{C}_{-}$, respectively. Furthermore, an approximation sequence to the fixed points can be generated, regardless of the chosen initial point.

Theorem 1. Let $G(s)$ be a damping function that satisfies the hypothesis H1, H2. Let $\left\{x_{n}\right\}$ be the sequence introduced in Eq. (2.13). Then:

i) The viscoelastic system given by Eq. (2.3) has a complex eigenvalue $\lambda \in \mathcal{C}_{+}$ if and only if there exists an initial $x_{0} \in \mathcal{C}_{+}$such that

$$
\lim _{n \rightarrow \infty} x_{n} \notin \mathbb{R}
$$

ii) Moreover, for all $z_{0} \in \mathcal{C}$, there exists a sequence $\left\{z_{n}\right\} \subset \mathcal{C}_{+}$, with $z_{1}=X\left(z_{0}\right)$ such that

$$
\lim _{n \rightarrow \infty} z_{n}=\lambda
$$

Proof. i) As a stratightforward result of the hypothesis, $\left\{x_{n}\right\}$ can not have accumulation points in $\mathbb{R}$. From property $\mathrm{P} 4, X\left(x_{n}\right) \in \mathcal{C}_{+}$holds, $\forall n \geq 0$ and $\forall x_{0} \in \mathcal{C}_{+}$. Consequently, there exists a real positive number $\epsilon>0$ such that $B\left(x_{n}, \epsilon\right) \subset \mathcal{C}_{+}$, for $n \geq 1$. Hence, it follows that the set $\left\{x_{n}\right\}_{n=1}^{\infty}$ lies strictly inside the set $\mathcal{C}_{+}$. 
To apply the Lemma 1 , the domain $\mathcal{C}_{+}$can be transformed into the open unit ball by the Cayley transformation $\psi: \mathcal{C}_{+} \rightarrow \mathbb{D}$, defined through the conformal mapping

$$
\psi(s)=\frac{i \omega_{u}-s}{i \omega_{u}+s}
$$

that transforms the upper half complex plane, $\mathcal{C}_{+}$into the open unit ball $\mathbb{D}$. For instance, the point $i \omega_{u}$ is transformed into the origin, and the boundary $\partial \mathcal{C}_{+}=\mathbb{R}$ into the circumference $\partial \mathbb{D}=\{w \in \mathbb{C}:|w|=1\}$. Now, let us define by composition the following complex function

$$
U=\psi \circ X \circ \psi^{-1}: \mathbb{D} \rightarrow \mathbb{D}
$$

where

$$
\psi^{-1}(w)=i \omega_{u} \frac{1-w}{1+w} \quad \in \mathcal{C}_{+} \forall w \in \mathbb{D}
$$

Immediately, $\psi, \psi^{-1}$ are holomorphic in $\mathcal{C}_{+}$and $\mathbb{D}$, respectively. Since $X$ is also holomorphic in $\mathcal{C}_{+} \subset \mathcal{A}$, it follows that $U$ is holomorphic in $\mathbb{D}$. Moreover, from property P3, it is verified that $X\left(\mathcal{C}_{+}\right) \subset \mathcal{C}_{+}$, and therefore $U(\mathbb{D}) \subset \mathbb{D}$. A sequence $\left\{w_{n}\right\}$ associated to $\left\{x_{n}\right\}$ can be generated, with elements that are images under $\psi$ in $\mathbb{D}$, i.e. $w_{n}=\psi\left(x_{n}\right) \in \mathbb{D}$ for $n \geq 0$. Let us verify that $w_{n}=U\left(w_{n-1}\right)$ holds, using the definition of $U(w)$

$$
\begin{aligned}
w_{n}=\psi\left(x_{n}\right) & =\psi\left(X\left(x_{n-1}\right)\right)=(\psi \circ X)\left(x_{n-1}\right) \\
& =(U \circ \psi)\left(x_{n-1}\right)=U\left(\psi\left(x_{n-1}\right)\right)=U\left(w_{n-1}\right)
\end{aligned}
$$

Since the complete sequence $\left\{x_{n}\right\}_{n=1}^{\infty}$ lies strictly inside $\mathcal{C}_{+}$, it is verified $\left\{w_{n}\right\}_{n=1}^{\infty} \subset$ $\mathbb{D}$ strictly. Therefore, from Lemma 1 there exists a fixed point $\gamma \in \mathbb{D}$ of the function $U$, i.e., $U(\gamma)=\gamma$, so that the anti-image $\lambda=\psi^{-1}(\gamma) \in \mathcal{C}_{+}$is a fixed point of $X$. Indeed, using again the definition of $U$

$$
\begin{aligned}
\lambda=\psi^{-1}(\gamma) & =\psi^{-1}(U(\gamma))=\left(\psi^{-1} \circ U\right)(\gamma) \\
& =\left(\psi^{-1} \circ \psi \circ X \circ \psi^{-1}\right)(\gamma)=X\left(\psi^{-1}(\gamma)\right)=X(\lambda)
\end{aligned}
$$

Finally, since the viscoelastic problem characterized by $G(s)$ has at most one eigenvalue in $\mathcal{C}_{+}$(see subsection 2.2.1), $\lambda$ is the unique fixed point of $X$ in $\mathcal{C}_{+}$. From the property $\mathrm{P} 3, \lambda^{*}=\psi^{-1}\left(\gamma^{*}\right)$ is a fixed point of the function $Y(s)$ and consequently the other complex eigenvalue of the viscoelastic system.

To finish the first part of the proof, let us consider reciprocally that $\lambda \in \mathcal{C}_{+}$is a complex eigenvalue of the viscoelastic system. Therefore, $\gamma=\psi(\lambda) \in \mathbb{D}$ is a fixed point of $U$. Applying Lemma $1,\left\{w_{n}\right\}_{n=1}^{\infty} \subset \mathbb{D}$ strictly and, hence

$$
\lim _{n \rightarrow \infty} x_{n}=\lim _{n \rightarrow \infty} \psi^{-1}\left(w_{n}\right) \notin \mathbb{R}
$$


ii) Assuming now that the system is not overdamped, there exists a unique eigenvalue $\lambda \in \mathcal{C}_{+}$that is fixed point of $X$. As shown in the previous proof, $\gamma=\psi(\lambda)$ is fixed point of the holomorphic self-mapping $U: \mathbb{D} \rightarrow \mathbb{D}$ defined in Eq. (2.15). Let $z_{0} \in \mathcal{C}$ be any complex number (excluding reals), from property $\mathrm{P} 4, z_{1}=X\left(z_{0}\right) \in$ $\mathcal{C}_{+}$and let $u_{1}=\psi\left(z_{1}\right) \in \mathbb{D}$. The following sequence

$$
u_{n}=\frac{u_{1}}{n^{\theta}}+\left(1-\frac{1}{n^{\theta}}\right) U\left(u_{n-1}\right)
$$

for $n \geq 2$ can be constructed in $\mathbb{D}$, where $\theta$ can be any real number in the open interval $0<\theta<1$. From Lemma 2, we can ensure that the sequence $\left\{u_{n}\right\}$ converges to $\gamma$. Consequently the sequence $\left\{z_{n}\right\}$ generated as

$$
z_{n}=\psi^{-1}\left(u_{n}\right)
$$

will converge to $\lambda$.

Theorem 1 allows us to ensure the existence of complex eigenvalues as fixed points of $X(s), Y(s)$. A recursive scheme towards the fixed point can be generated from Eqs. (2.20), (2.21), but it is not established whether the sequences of iterates $\left\{x_{n}\right\}$, $\left\{y_{n}\right\}$ from Eq. (2.13) converge always to eigenvalues. It seems logical to assume that when $\left\{z_{n}\right\}$ converges also does $\left\{x_{n}\right\}$, but it is an unproven conjecture. In fact, if the conditions of Theorem 1 are satisfied, theoretically a fixed point of $X(s)$ could coexist with a non-convergent sequence $\left\{x_{n}\right\}$. In such case, the recursive scheme would lie in an infinite non-convergent loop contained strictly inside $\mathbb{D}$. However, the authors have not found any case with this behavior. Furthermore, for all analyzed cases the sequence $\left\{x_{n}\right\}$ has converged successfully to the complex eigenvalue for any considered initial point $x_{0} \in \mathcal{C}_{+}$. An explicit proof for this convergence is not currently available when the requirements of the function $J(s)$ are just the hypothesis $\mathrm{H} 1, \mathrm{H} 2$.

Despite this formal lack of proof and motivated by a justification for the efficiency of the numerical scheme $\left\{x_{n}\right\}$, new additional conditions on the function $J(s)$ are going to be introduced. First, the following Theorem 2 proves local convergence to fixed points of $X(s), Y(s)$. Second, Theorem 3 establishes the necessary conditions to ensure global convergence of sequences $\left\{x_{n}\right\},\left\{y_{n}\right\}$ to fixed points in a closed ball centered at the undamped eigenvalues $\pm i \omega_{u}$, respectively. Moreover, these theorems will allow us to predict the speed of convergence, aspect not possible just with Theorem 1. The statement and proofs of these theorems are presented for the function $X(s)$. The application for $Y(s)$ can be obtained as a direct consequence of properties $\mathrm{P} 1$ to P5. 
Theorem 2. Let $\lambda \in \mathcal{C}_{+}$be the complex eigenvalue of the viscoelastic system (2.3). If

$$
\left|\frac{\partial J(\lambda)}{\partial s}\right|<\left|\frac{1}{\omega_{u}}+\frac{J(\lambda)}{\lambda}\right|
$$

then there exist two positive real numbers $\delta>0,0<\rho<1$, such that the sequence $\left\{x_{n}\right\}$ converges to $\lambda$ for any initial point $x_{0} \in B(\lambda, \delta)=\{s \in \mathbb{C}:|s-\lambda| \leq \delta\}$. Furthermore,

$$
\left|x_{n}-\lambda\right| \leq \frac{\rho^{n}}{1-\rho}\left|X\left(x_{0}\right)-x_{0}\right|
$$

Proof. The proof is based on verification of the function $X(s)$ satisfying Banach's contraction mapping principle $[67,68]$. As well known, this principle constitutes one of the most important results in mathematical analysis. Applied to the complex domain, it states that every contraction self-mapping on a closed set has a unique fixed point. Moreover, the principle allows the bounding of the computed error after $n$ iterations. The proof of this theorem can be organized into two steps:

i) $X(s)$ is contractive in the closed ball $B(\lambda, \delta)$. The derivative of $X(s)$ from Eqs. (2.10) is

$$
\frac{\partial X}{\partial s}=-\frac{\partial J}{\partial s}\left(1+\frac{i J(s)}{\sqrt{1-J^{2}(s)}}\right) \omega_{u}
$$

Using the inequality given by Eq. (2.22) and $X(\lambda)=\lambda$

$$
\begin{aligned}
\left|\frac{\partial X(\lambda)}{\partial s}\right| & =\left|\frac{\partial J(\lambda)}{\partial s}\right|\left|1+\frac{i J(\lambda)}{\sqrt{1-J^{2}(\lambda)}}\right| \omega_{u}=\left|\frac{\partial J(\lambda)}{\partial s}\right|\left|\frac{-J(\lambda)+i \sqrt{1-J^{2}(\lambda)}}{i \sqrt{1-J^{2}(\lambda)}}\right| \omega_{u} \\
& =\left|\frac{\partial J(\lambda)}{\partial s}\right|\left|\frac{X(\lambda)}{\frac{X(\lambda)}{\omega_{u}}+J(\lambda)}\right|=\left|\frac{\partial J(\lambda)}{\partial s}\right|\left|\frac{\lambda}{\frac{\lambda}{\omega_{u}}+J(\lambda)}\right|=\frac{\left|\frac{\partial J(\lambda)}{\partial s}\right|}{\left|\frac{1}{\omega_{u}}+\frac{J(\lambda)}{\lambda}\right|}<1
\end{aligned}
$$

Since $X(s)$ is analytical at $s=\lambda \in \mathcal{C}_{+}$, the absolute value of its derivative, $|\partial X / \partial s| \equiv\left|X^{\prime}(s)\right|$ is continuous in a neighborhood of $\lambda$. Then, there exist a positive real number $\delta>0$ so that the maximum value in $B(\lambda, \delta)$ is

$$
\rho=\max _{|s-\lambda| \leq \delta}\left|\frac{\partial X(s)}{\partial s}\right|<1
$$

In real analysis, the previous conclusion is sufficient to prove that $X(s)$ is contractive by straight application of the mean value theorem. In complex analysis this theorem can not be applied in the same terms, but an analog for analytical complex functions was demonstrated by McLeod [69]: let $u, v \in B(\lambda, \delta)$ be two any 
complex numbers. Since the ball is obviously a convex set, the complex segment between them is $[u, v]=\{\xi u+(1-\xi) v: 0 \leq \xi \leq 1\} \subset B(\lambda, \delta)$. According to the reference [69], there exists a real number $0 \leq \xi_{0} \leq 1$ and two complex numbers $s_{1}, s_{2} \in[u, v]$ such that

$$
X(u)-X(v)=(u-v)\left[\xi_{0} \frac{\partial X\left(s_{1}\right)}{\partial s}+\left(1-\xi_{0}\right) \frac{\partial X\left(s_{2}\right)}{\partial s}\right]
$$

Consequently, taking the absolute value of Eq. (2.27) and using the bound given by Eq. (2.26), the Lipschitz continuity of $X(s)$ can be proved by

$$
\begin{aligned}
|X(u)-X(v)| & =\left|(u-v)\left[h \frac{\partial X\left(s_{1}\right)}{\partial s}+(1-h) \frac{\partial X\left(s_{2}\right)}{\partial s}\right]\right| \\
& \leq|u-v|\left[h\left|\frac{\partial X\left(s_{1}\right)}{\partial s}\right|+(1-h)\left|\frac{\partial X\left(s_{2}\right)}{\partial s}\right|\right] \\
& \leq[h \rho+(1-h) \rho]|u-v|=\rho|u-v|
\end{aligned}
$$

Since the Lipschitz coefficient verifies $\rho<1$, the function $X(s)$ is contractive.

ii) $X(s)$ is a self-mapping if given any $s \in B(\lambda, \delta)$ i.e. $|s-\lambda| \leq \delta$, its image $X(s)$ also lies inside the ball, i.e. $X(s) \in B(\lambda, \delta)$ or equivalently $|X(s)-\lambda| \leq \delta$. The previous inequality can be proved just using the contraction property in $B(\lambda, \delta)$

$$
|X(s)-\lambda|=|X(s)-X(\lambda)| \leq \rho|s-\lambda| \leq \rho \delta<\delta
$$

Therefore, the hypotheses from Banach's fixed point theorem, see [68], are satisfied and the convergence of the sequence $\left\{x_{n}\right\}$ from Eq. (2.13) to the (unique) fixed point of $X(s)$ in the ball $B(\lambda, \delta)$ is ensured. Furthermore, the error decay rate in each iteration can be bounded by

$$
\left|x_{n}-\lambda\right| \leq \frac{\rho^{n}}{1-\rho}\left|X\left(x_{0}\right)-x_{0}\right|, \quad \forall x_{0} \in B(\lambda, \delta)
$$

In view of the previous results, it can be demonstrated, see [70], that the speed of convergence of a fixed point iteration scheme is asymptotically linear. In fact, it is verified that

$$
\lim _{n \rightarrow \infty} \frac{\left|x_{n+1}-\lambda\right|}{\left|x_{n}-\lambda\right|}=\rho<1
$$

Notice that the hypotheses of Theorem 2 are expressed in terms of the unknown eigenvalue $\lambda$. Therefore, there is no information a priori on the error rate $\left|x_{n}-\lambda\right|$ since $\rho$ depends on the unknown $\left|X^{\prime}(\lambda)\right|$. In order to improve this theorem, the 
subsequent Theorem 3 demonstrates the convergence of $\left\{x_{n}\right\}$ using again Banach's contraction principle, but now in a closed ball centered in the undamped eigenvalue $i \omega_{u}$. In addition, this theorem will allow us to relate the speed of convergence with the damping model characteristics, giving a physical insight into the mathematical results. For that, let us introduce two preliminary definitions: given a positive real number $r>0$, and let $\mathcal{H}_{r}=\left\{s \in \mathbb{C}:\left|s-i \omega_{u}\right| \leq r\right\}$ be the closed ball centered in $i \omega_{u}$ with radius $r$. The following numbers are bounds defined as

$$
\alpha_{r}=\max _{s \in \mathcal{H}_{r}}|J(s)|, \quad \beta_{r}=\omega_{u} \max _{s \in \mathcal{H}_{r}}\left|\frac{\partial J}{\partial s}\right|
$$

The existence of $\alpha_{r}, \beta_{r}$ is guaranteed provided that $\mathcal{H}_{r}$ lies inside the analyticity domain $\mathbb{C} \backslash \mathcal{M}$ of $J(s)$. The presence of $\mathcal{H}_{r}$ allows us to express the necessary conditions of Theorem 3 in terms of $\alpha_{r}, \beta_{r}$.

Theorem 3. Let $\alpha_{r}, \beta_{r}$ be the bounds defined by Eq. (2.32). If

$$
\alpha_{r}<1, \quad \beta_{r}\left(1+\frac{\alpha_{r}}{\sqrt{1-\alpha_{r}^{2}}}\right) \equiv \kappa_{r}<1, \quad \frac{\left|X\left(i \omega_{u}\right)-i \omega_{u}\right|}{r\left(1-\kappa_{r}\right)} \leq 1
$$

then there exists an unique fixed point $\lambda$ of $X(s)$ in the set $\mathcal{H}_{r}$ and the rate of convergence of the sequence $x_{n}=X\left(x_{n-1}\right)$ is

$$
\left|x_{n}-\lambda\right| \leq \frac{\kappa_{r}^{n}}{1-\kappa_{r}}\left|X\left(x_{0}\right)-x_{0}\right|, \quad \forall x_{0} \in \mathcal{H}_{r}
$$

Proof. Since the domain $\mathcal{H}_{r} \subset \mathbb{C}$ is a closed set in the complex domain, it is a complete set. To apply again Banach's contraction principle to the function $X: \mathcal{H}_{r} \rightarrow \mathbb{C}$, the proof is again organized in two steps.

i) The function $X(s)$ is contractive in the set $\mathcal{H}_{r}$. From Eq. (2.32), (2.34) left, $|J(s)| \leq \alpha_{r}<1$ for any $s \in \mathcal{H}_{r}$. Consequently, the ball $\mathcal{H}_{r}$ lies inside the region where $X(s)$ is analytic, that is, $\mathcal{H}_{r} \subset \mathcal{A}=\mathbb{C} \backslash(\mathcal{M} \cup \mathcal{B})$. The contractivity of $X(s)$ can be proved as in Theorem 2, thus, it is only required that the bound of $\left|X^{\prime}(s)\right|$ is strictly less than one in $\mathcal{H}_{r}$. Using the definitions from Eq. (2.32) and Eq. (2.24) we have $\forall s \in \mathcal{H}_{r}$ 


$$
\begin{aligned}
\left|\frac{\partial X}{\partial s}\right| & =\omega_{u}\left|\frac{\partial J}{\partial s}\right|\left|1+\frac{i J(s)}{\sqrt{1-J^{2}(s)}}\right| \leq \beta_{r}\left(1+\left|\frac{i J(s)}{\sqrt{1-J^{2}(s)}}\right|\right) \\
& =\beta_{r}\left(1+|J(s)|\left|\sum_{k=0}^{\infty}\left(\begin{array}{c}
-1 / 2 \\
k
\end{array}\right)(-1)^{k} J^{2 k}(s)\right|\right) \\
& \leq \beta_{r}\left(1+\alpha_{r} \sum_{k=0}^{\infty}\left|\left(\begin{array}{c}
-1 / 2 \\
k
\end{array}\right)\right||J(s)|^{2 k}\right) \\
& \leq \beta_{r}\left(1+\alpha_{r} \sum_{k=0}^{\infty}\left(\begin{array}{c}
-1 / 2 \\
k
\end{array}\right)(-1)^{k} \alpha_{r}^{2 k}\right) \\
& =\beta_{r}\left(1+\frac{\alpha_{r}}{\sqrt{1-\alpha_{r}^{2}}}\right)=\kappa_{r}<1
\end{aligned}
$$

where the last inequality holds from Eq. (2.33) middle. In the previous development, $\alpha_{r}<1$ has been used to express the bound of $1 / \sqrt{1-J^{2}(s)}$ in terms of $\alpha_{r}$ through its series expansion. It can be concluded the complex function $X(s)$ is contractive in the set $\mathcal{H}_{r}$.

ii) The function $X(s)$ is a self-mapping of the set $\mathcal{H}_{r}$, i.e., $X\left(\mathcal{H}_{r}\right) \subset \mathcal{H}_{r}$. We focus now on the verification of $X(s) \in \mathcal{H}_{r}, \forall s \in \mathcal{H}_{r}$. Thus, let us consider a $s \in \mathbb{C}$ such that $\left|s-i \omega_{u}\right| \leq r$, hence, $X(s) \in \mathcal{H}_{r}$ holds if $\left|X(s)-i \omega_{u}\right| \leq r$. Using the hypothesis from Eq. (2.33) right, it follows

$$
\begin{aligned}
\left|X(s)-i \omega_{u}\right| & =\left|X(s)-X\left(i \omega_{u}\right)+X\left(i \omega_{u}\right)-i \omega_{u}\right| \\
& \leq\left|X(s)-X\left(i \omega_{u}\right)\right|+\left|X\left(i \omega_{u}\right)-i \omega_{u}\right| \\
& \leq \kappa_{r}\left|s-i \omega_{u}\right|+\left|X\left(i \omega_{u}\right)-i \omega_{u}\right| \\
& \leq \kappa_{r} r+\left|X\left(i \omega_{u}\right)-i \omega_{u}\right| \\
& \leq \kappa_{r} r+\left(1-\kappa_{r}\right) r=r
\end{aligned}
$$

In summary, the function $X(s)$ and the ball $\mathcal{H}_{r}$ verifiy the following three properties

- $\mathcal{H}_{r}$ is closed and therefore a complete subset in the complex domain

- $X\left(\mathcal{H}_{r}\right) \subset \mathcal{H}_{r}$

- $X(s)$ is contractive in $\mathcal{H}_{r}$ with Lipschitz coefficient $\kappa_{r}<1$ 
Therefore, the Banach's contraction principle can to be applied again for $X(s)$, now in $\mathcal{H}_{r}$ assuring the uniqueness of the fixed point $\lambda \in \mathcal{H}_{r}$. Furthermore, the speed of convergence of the sequence $x_{n} \rightarrow \lambda$ is linear and the error can be bounded by

$$
\left|x_{n}-\lambda\right| \leq \frac{\kappa_{r}^{n}}{1-\kappa_{r}}\left|X\left(x_{0}\right)-x_{0}\right|, \quad \forall x_{0} \in \mathcal{H}_{r}
$$

Notice that no reference to the eigenvalue $\lambda$ in the three hypotheses has been made, then, given a radius $r>0$, they can easily be tested a priori provided that $\alpha_{r}$, $\beta_{r}$ are available. These bounds depend on the mathematical form of the damping function $J(s)$ and they must be searched in the boundary of $\mathcal{H}_{r}$, according to the maximum modulus theorem for analytical complex functions. Assuming now that the necessary conditions of Theorem 3 are satisfied, the mathematical expression of the Lipschitz coefficient

$$
\kappa_{r}=\beta_{r}\left(1+\frac{\alpha_{r}}{\sqrt{1-\alpha_{r}^{2}}}\right)
$$

contains relevant qualitative information. On one hand, as shown in Theorem $3, \kappa_{r}$ controls the convergence speed. On the other, its expression, given by Eq. (2.38), depends directly on the bounds of the (dimensionless) viscoelastic function $J(s)$ and its derivative $J^{\prime}(s)$. The physical meaning of these values is directly related with the characteristics of the damping model: a) $\alpha_{r}$ is the damping function (in absolute value) maximum in $\mathcal{H}_{r}$. Low values of $J(s)$ are associated with lightly damped systems and $\alpha_{r}$ is a measure of the level of damping; b) $\beta_{r} / \omega_{u}$ directly represents the bound of $J^{\prime}(s)$, derivative directly related with the level of viscoelasticity. Mathematically, a system has small viscoelasticity if the damping function $J(s)$ does not present large variations with respect to $s$ so that $\beta_{r}$ measures the viscoelasticity in $\mathcal{H}_{r}$. A more complete study of the viscoelasticity quantification may be found in the work of Adhikari and Woodhouse [71]. Since the real valued function $\kappa(\alpha, \beta)=\beta\left(1+\alpha / \sqrt{1-\alpha^{2}}\right)$ is always increasing in the range $\beta>0$, $0<\alpha<1$, it is expected that lightly damped systems with low viscoelasticity will present faster convergence to the fixed points. Hence, the higher the damping level in the system the slower the convergence to the eigenvalue using the proposed recursive method. This affirmation will be validated throughout the numerical examples. 


\subsubsection{Real Eigenvalues}

Assuming that the damping function $G(s)$ induces a dissipative motion, energy loss may result in either a decreasing amplitude oscillatory motion (complex eigenvalues) or a non-oscillatory motion with exponential decay (real negative eigenvalues). For the first, the system is said to be underdamped and for the second overdamped. In the latter no complex number in the set $\mathcal{C}=\mathbb{C} \backslash \mathbb{R}$ exits as fixed point of the functions $X(s), Y(s)$. Therefore, as a corollary of Theorem 1 both recursive sequences will converge to the same real eigenvalue. Starting from a point $x_{0} \in \mathcal{C}_{+}$the elements of $\left\{x_{n}\right\}$ will be contained in the upper half-plane of the complex domain and will converge to a negative number. At the same time, $\left\{y_{n}\right\}$ will also converge to the same eigenvalue but through the lower half-plane starting now in $x_{0}^{*} \in \mathcal{C}_{-}$. However, in underdamped systems non-viscous real eigenvalues coexist with the complex conjugate eigenvalues pair and the question arises whether the recursive functions, $X(s), Y(s)$ are able to converge to some real eigenvalue.

Property P5 of the recursive functions states that $X(\mathcal{B}) \subset \mathbb{R}$ and $Y(\mathcal{B}) \subset \mathbb{R}$, provided that $J(\mathbb{R}) \subset \mathbb{R}$. Therefore, the functions $X, Y: \mathcal{B} \rightarrow \mathbb{R}$ are well defined and real valued. Obviously, any real eigenvalue must be a fixed point of some of the recursive functions, $X$ or $Y$. Once more, the fixed point theory provides a result due to Schröeder [70, 72] giving the necessary conditions for the convergence (local in this case), to a fixed point. This theorem states that the sequence of iterates $t_{n}=X\left(t_{n-1}\right)$ is locally convergent to a fixed point $\sigma \in \mathbb{R}$ if the derivative fulfills $|\partial X(\sigma) / \partial t|<1$. The same conclusion can be established for the function $Y(t)$. The notation $t$ is used for the real independent variable and should not be confused with time, which only appears in Eq. (2.3). Since $X(t)$ is continuously differentiable in a neighborhood of $\sigma$, there exists an interval centered in this point, $I=[\sigma-\delta, \sigma+\delta]$ such that

$$
\rho=\max _{t \in I}\left|\frac{\partial X(t)}{\partial t}\right|<1
$$

It can be easily proved that any sequence $\left\{t_{n}\right\}_{n=0}^{\infty}$ that starts in the interval $I$ remains in $I$. Thus, the difference between the $n$th term and the fixed point can be bounded by

$$
\left|\sigma-t_{n}\right|=\left|X(\sigma)-X\left(t_{n-1}\right)\right| \leq \rho\left|\sigma-t_{n-1}\right| \leq \cdots \leq \rho^{n}\left|\sigma-t_{0}\right|
$$

which proves the convergence, $\lim _{n \rightarrow \infty} x_{n}=\sigma$. This theorem can be considered as a weak version of the Banach's contraction principle in the real domain and, like Theorem 2, does not provide practical hypotheses due to its dependence on the (unknown) fixed point. However, the numerical examples will show that, under certain conditions, $X(t), Y(t)$ can present low derivative values. Consequently, testing the convergence of the iterative process starting from some point $t_{0} \in \mathbb{R}$, would permit to find non-viscous eigenvalues. 


\subsection{Multiple Degree-of-Freedom Systems}

The main objective of this section is to apply the previously obtained results for single dof systems to multiple ones. Let us name $\mathbf{M} \in \mathbb{R}^{q \times q}, \mathbf{K} \in \mathbb{R}^{q \times q}$, $\mathbf{u}(t) \in \mathbb{R}^{q}$ to the mass and to the elastic stiffness matrices, and to the vector grouping the dof's. Damping in non-viscous (or viscoelastic) dynamic systems is modeled by dissipative forces that depend on the history of the dof velocities via kernel functions. The equilibrium of the free motion results in a system of integro-differential equations that can be expressed in matrix form as follows

$$
\mathbf{M} \ddot{\mathbf{u}}+\int_{-\infty}^{t} \mathcal{G}(t-\tau) \dot{\mathbf{u}} \mathrm{d} \tau+\mathbf{K u}=\mathbf{0}
$$

where the matrix $\mathcal{G}(t) \in \mathbb{R}^{q \times q}$ contains the viscoelastic kernel functions in time domain. Testing solutions of the form $\mathbf{u}(t)=\mathbf{u}_{0} e^{s t}$, the previous system becomes the nonlinear eigenvalue problem

$$
\left[s^{2} \mathbf{M}+s \mathbf{G}(s)+\mathbf{K}\right] \mathbf{u}_{0}=\mathbf{0}
$$

where $\mathbf{G}(s)=\mathcal{L}\{\mathcal{G}(t)\} \in \mathbb{C}^{q \times q}$. Let $\boldsymbol{\Phi}=\left[\phi_{1}, \ldots, \phi_{q}\right] \in \mathbb{R}^{q \times q}$ be the matrix grouping the eigenvectors associated with the undamped system defined by matrices $\mathbf{M}$, K. The classical orthogonal relations can be written as

$$
\boldsymbol{\Phi}^{T} \mathbf{M} \boldsymbol{\Phi}=\tilde{\mathbf{M}}=\operatorname{diag}\left[m_{j}\right], \quad \boldsymbol{\Phi}^{T} \mathbf{K} \boldsymbol{\Phi}=\tilde{\mathbf{K}}=\operatorname{diag}\left[k_{j}\right]
$$

Changing the variable $\mathbf{u}_{0}=\mathbf{\Phi} \mathbf{z}_{0}$ and using the orthogonal relations, Eq. (2.42) may be expressed as

$$
\left[s^{2} \tilde{\mathbf{M}}+s \boldsymbol{\Gamma}(s)+\tilde{\mathbf{K}}\right] \mathbf{z}_{0}=\mathbf{0}
$$

where $\boldsymbol{\Gamma}(s)=\boldsymbol{\Phi}^{T} \mathbf{G}(s) \boldsymbol{\Phi}$ is the damping matrix in the modal space. In general, this matrix is not diagonal, only non-viscous proportional damping presents this property. The necessary and sufficient conditions for proportional damping in non-viscous systems have been studied by Adhikari [29]. For the current paper purposes, the hypothesis of light non-proportional damping is considered, i.e., the matrix $\boldsymbol{\Gamma}\left(\lambda_{j}\right)$ is diagonally dominant, that is equivalent to assume as true that

$$
\sum_{\substack{l=1 \\ l \neq k}}^{q}\left|\Gamma_{k l}\left(\lambda_{j}\right)\right|<\left|\Gamma_{k k}\left(\lambda_{j}\right)\right|, \quad \forall 1 \leq k \leq q
$$

where $\lambda_{j}$ is the $j$ th eigenvalue. This assumption is commonly assumed in many problems related with non-viscous damping [28, 30-32] and it allows the approximation of the determinant as the product of the terms of its main diagonal, that is

$$
\operatorname{det}\left[s^{2} \tilde{\mathbf{M}}+s \boldsymbol{\Gamma}(s)+\tilde{\mathbf{K}}\right] \approx \prod_{j=1}^{q}\left(m_{j} s^{2}+s \Gamma_{j j}(s)+k_{j}\right)
$$


Hence, the set of eigenvalues can be obtained from the following $q$ equations

$$
D_{j}(s)=m_{j} s^{2}+s \Gamma_{j j}(s)+k_{j}=0, \quad 1 \leq j \leq q
$$

In general, the number of eigenvalues of an oscillatory viscoelastic (underdamped) system is $2 q+p$, with $p \geq 0$; there are $2 q$ complex eigenvalues formed by $q$ complex conjugate pairs. In addition, the set of eigenvalues must be completed with $p$ real and non-viscous roots. This set is formed by negative numbers associated with overcritical modes, of non-oscillatory nature. The total number of non-viscous roots can be written as $p=p_{1}+\cdots+p_{q}$, where $p_{j} \geq 0,1 \leq j \leq q$ is the number of non-viscous roots of the $j$ th characteristic equation.

In order to apply the recursive scheme, let us define the dimensionless damping functions

$$
J_{j}(s)=\frac{\Gamma_{j j}(s)}{2 m_{j} \omega_{j}}, \quad 1 \leq j \leq q
$$

Following the same procedure as before, the $j$ th equation can be expressed as

$$
\frac{D_{j}(s)}{m_{j}}=s^{2}+2 s J_{j}(s) \omega_{j}+\omega_{j}^{2}=\left[s-X_{j}(s)\right]\left[s-Y_{j}(s)\right]
$$

where

$$
\begin{aligned}
& X_{j}(s)=\omega_{j}\left(-J_{j}(s)+i \sqrt{1-J_{j}^{2}(s)}\right) \\
& Y_{j}(s)=\omega_{j}\left(-J_{j}(s)-i \sqrt{1-J_{j}^{2}(s)}\right)
\end{aligned}
$$

are the functions used in recursive form to compute the complex eigenvalues. The fixed point theory provides a sequence defined by Eq. (2.20) contained in the complex unit ball that strongly converges to the fixed point. However, the sequences

$$
x_{n}=X_{j}\left(x_{n-1}\right), \quad y_{n}=Y_{j}\left(y_{n-1}\right), \quad n \geq 1
$$

behave very well and in practice can efficiently be used to obtain the fixed point. As described in the previous section, the speed of convergence depends on damping level and on viscoelasticity. As the starting point of the algorithm, the undamped eigenvalue $i \omega_{j}$ is in general a good choice; however any suitable initial point can be used as long as it belongs to $\mathcal{C}=\mathbb{C} \backslash \mathbb{R}$.

Finally, for the eigenvectors' computation, two procedures can be used [28]. From the approximated eigenvalue obtained with the recursive process described above $\lambda_{j}$, the components of the $j$ th associated eigenvector, say $\mathbf{u}_{j}$, can be computed. First, from the ill-conditioned linear system of equations

$$
\left[\lambda_{j}^{2} \mathbf{M}+\lambda_{j} \mathbf{G}\left(\lambda_{j}\right)+\mathbf{K}\right] \mathbf{u}_{j}=\mathbf{0}
$$


This method can be computationally inefficient for large size systems because it involves the calculation of an inverse submatrix of dimension $q-1$; in any case it is specially recommended for non-viscous eigenvectors associated to nonviscous eigenvalues, for which no alternative method is currently available. Second, for the complex eigenvectors, the hypothesis of non-proportional damping allows the use of approximate expressions. The complex eigenvectors are expanded in terms of the off-diagonal terms of matrix $\boldsymbol{\Gamma}\left(\lambda_{j}\right)=\boldsymbol{\Phi}^{T} \mathbf{G}\left(\lambda_{j}\right) \boldsymbol{\Phi}$. Let us name $\mathcal{D}_{j}(s)=D_{j}(s) / \sqrt{m_{j}}$ to the expression given by Eq. (2.49) and $\boldsymbol{\psi}_{j}=\boldsymbol{\phi}_{j} / m_{j}$ to the mass-normalized $j$ th undamped eigenvector, for $1 \leq j \leq q$. The second order approximation of the $j$ th eigenvector is expressed as linear combination of the undamped eigenvectors $\left\{\boldsymbol{\psi}_{1}, \ldots, \boldsymbol{\psi}_{q}\right\}$, which forms a base of the space $\mathbb{R}^{q}$.

$$
\mathbf{u}_{j} \approx \boldsymbol{\psi}_{j}-\lambda_{j} \sum_{\substack{k=1 \\ k \neq j}}^{q} \frac{\Gamma_{k j}\left(\lambda_{j}\right)}{\mathcal{D}_{k}\left(\lambda_{j}\right)} \boldsymbol{\psi}_{k}+\lambda_{j}^{2} \sum_{\substack{k=1 \\ k \neq j}}^{q} \sum_{\substack{l=1 \\ l \neq j \neq k}}^{q} \frac{\Gamma_{k l}\left(\lambda_{j}\right) \Gamma_{l j}\left(\lambda_{j}\right)}{\mathcal{D}_{k}\left(\lambda_{j}\right) \mathcal{D}_{l}\left(\lambda_{j}\right)} \boldsymbol{\psi}_{k}
$$

\section{$2.4 \quad$ Numerical Examples}

\subsubsection{Example 1: Single Degree-of-Freedom Systems}

In order to validate the theoretical developments a single dof system is analyzed. For that, the Biot's viscoelastic model [7] with one exponential kernel as the hereditary damping function is used.

$$
\mathcal{G}(t)=c_{v} \mu e^{-\mu t}
$$

where $\mu>0$ is the relaxation parameter and $c_{v}$ the damping coefficient of the limit viscous model when $\mu \rightarrow \infty$. Let us denote by $\zeta=c_{v} / 2 m \omega_{u}$ the damping ratio of the limit viscous model, then the Laplace transform of the damping function is

$$
G(s)=2 m \omega_{u} \zeta \frac{\mu}{s+\mu}
$$

The characteristic equation is of the form

$$
s^{2}+2 s \omega_{u} \zeta \frac{\mu}{s+\mu}+\omega_{u}^{2}=0
$$

Multiplying the previous equation by $s+\mu$, it is transformed into a third order polynomial. Introducing now the new variables $z=s / \omega_{u}, \nu=\omega_{u} / \mu$ the following non-dimensional equation is obtained

$$
(\nu z+1)\left(z^{2}+1\right)+2 z \zeta=0
$$


Several authors $[55,64,73,74]$ have studied the dynamics of the described viscoelastic oscillator through the solutions of the previous polynomial. The three roots will be either a complex conjugate pair together with a single real number or three negative real numbers. The so-called overdamped region is defined by the set of damping parameters $\nu, \zeta$ that induce an overdamped motion, i.e. Eq. (2.57) gives three negative real roots. Adhikari [74] carried out a rigorous analysis of the $\nu, \zeta$ domain. Among other properties, the reference obtained closed expressions of the region boundary in terms of $\nu, \zeta$. In Fig. 2.1 left the representation of the overdamped (OD) region is drawn as a grey area. The rest (white area) will be the underdamped region (UD).

The availability of an explicitly defined overdamped region has motivated us to adopt Biot's damping model with one kernel. Convergence properties of the proposed recursive method and damping characteristics will be related. By means of straightforward operations, it can be verified that the particular function $J(s)=$ $\zeta \mu /(s+\mu)$ satisfies H1, H2. If the sequence $x_{n}=X\left(x_{n-1}\right)$ does not converge to the set $\mathbb{R}$ for some initial point $x_{0} \in \mathcal{C}_{+}$, then Theorem 1 ensures the existence of a complex fixed point of the function $X(s)$. Furthermore, if the damping model induces an overdamped motion, the sequence converges to a real eigenvalue but the theorem does not give information about the convergence speed. At this regard, Theorems 2 and 3 state that the Banach's contraction principle can be applied under certain conditions. The theoretical results have shown that lightly damped systems with low viscoelasticity present faster convergence to the eigenvalue.

Given a pair $(\zeta, \nu)$ the recursive scheme $x_{n}=X\left(x_{n-1}\right)$ can be carried out to approximate the eigenvalue, starting always from the initial point $x_{0}=i \omega_{u}$. Since $y_{n}=x_{n}^{*}$, the sequence $\left\{y_{n}\right\}$ is not of interest provided that $y_{0}=x_{0}^{*}$. It is considered that the method has reached a solution when the error defined by

$$
\epsilon_{n}=\left|\frac{x_{n}-x_{n-1}}{x_{1}-x_{0}}\right|
$$

is lower than the prefixed value $\epsilon_{\max }=10^{-12}$. If $\lambda(\zeta, \nu)$ is the eigenvalue obtained as the limit of $\left\{x_{n}\right\}$, the density plot of $\left|X^{\prime}(\lambda(\zeta, \nu))\right|$ is drawn in Fig. 2.1 right for $\omega_{u}=10 \mathrm{rad} / \mathrm{s}$. It should be noted that for all pairs (points in the plot) the sequence is convergent since the absolute value of the derivative is always less than the unity. The lowest values of $\left|X^{\prime}(\lambda(\zeta, \nu))\right|$ are located in the UD area with factor $0<\zeta<1$ and in the OD region. Therefore, it is expected that the recursive method is faster in these zones whereas the derivative values close to one (on the OD contour) are related with very slow convergence. To illustrate the convergence of the different zones, nine different cases associated with points $\mathrm{C} 1$ to $\mathrm{C} 9$ represented in Fig. 2.1 left. 


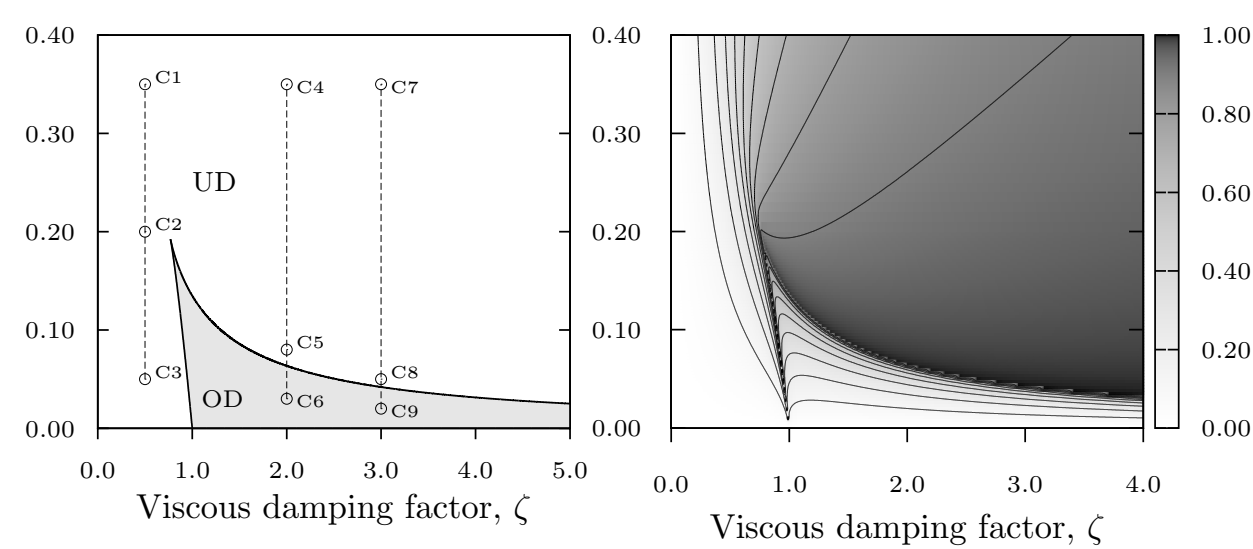

Figure 2.1: Left: underdamped UD, overdamped OD regions in parametric domain $(\zeta, \nu)$ of Example 1 and points $\mathrm{C} 1$ to $\mathrm{C} 9$ defined for numerical cases. Right: density plot of $\left|X^{\prime}(\lambda(\zeta, \nu))\right|$ for complex eigenvalue $\lambda(\zeta, \nu)$.

In Table 2.2 the approximated eigenvalue $x_{N}$ ( $N$ is the number of iterations for $\epsilon_{N}<\epsilon_{\max }$ ) obtained from the recursive sequence are listed for the nine cases in the fourth column. Due to the special relevance of the recursive function derivative $X^{\prime}(s)=\partial X / \partial s$, its absolute value evaluated in $x_{N}$ and in the undamped eigenvalue are listed in the last two columns. As expected, C5, C8 require a great number of iterations since the related values of $\left|X^{\prime}(\lambda)\right|$ are close to one. On the contrary, low values of $\left|X^{\prime}(\lambda)\right|$ as for $\mathrm{C} 1, \mathrm{C} 2, \mathrm{C} 3, \mathrm{C} 6, \mathrm{C} 9$ produce very fast convergence. Points C6, C9 converge to real eigenvalues since they are inside the OD. The number of iterations for $\mathrm{C} 4, \mathrm{C} 7$ is one order of magnitude higher due to the closeness of $\left|X^{\prime}(\lambda)\right|$ to unity.

In Fig. 2.2, (first nine plots) the complex-domain paths of the computed sequences have been represented together (last three plots) with the error-iterations plots for each numerical case. The initial guess $x_{0}$ is signaled by a circle and the converged eigenvalue by a square. It can be observed that Cases C1to C3 need few iterations to approach a neighborhood of the final eigenvalue. In these cases, the Theorem 3 can be applied and a suitable value of radius $r$ could be found. In the rest of cases, the sequences always converge but the speed of convergence could be predicted only in a neighborhood of the eigenvalue by means of Theorem 2 .

To complete this example, a few remarks on the application of the method to non-viscous eigenvalues computation will be made. As known, in a context of oscillatory motion a single dof viscoelastic model can have real eigenvalues ( non- $^{-}$ viscous eigenvalues), as well as a single complex conjugate pair. In subsection 2.2.3 it has been demonstrated that under certain conditions the sequences $\left\{x_{n}\right\},\left\{y_{n}\right\}$ 

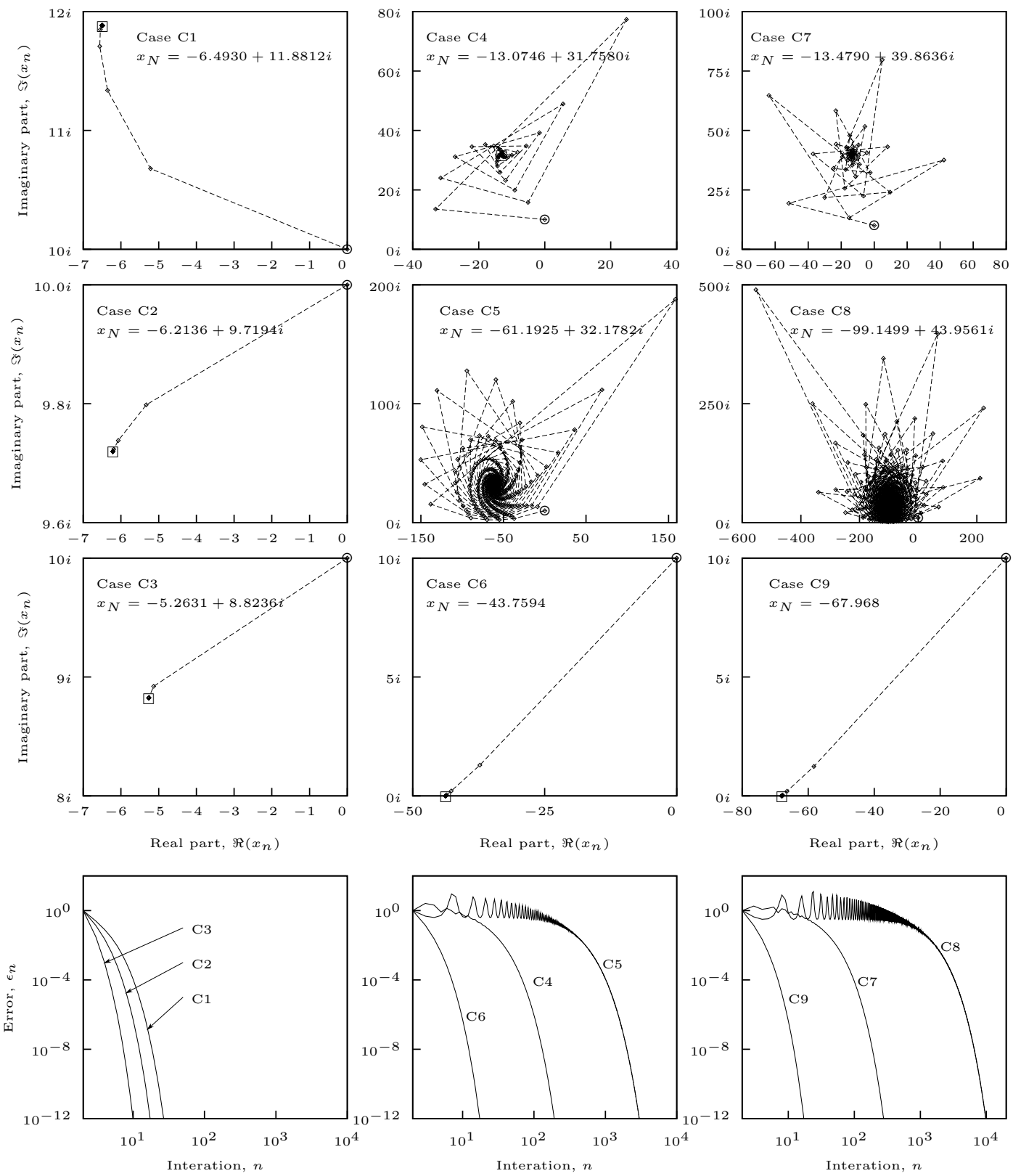

Figure 2.2: Example 1: Complex path of recursive sequence $x_{n}$ from initial guess $x_{0}=i \omega_{u}=10 i$, point $\odot$, up to the limit eigenvalue $\odot$, top nine plots. Associated error-iteration $\epsilon_{n}$ curves, bottom three plots, all for cases in Fig. 2.1 left. 


\begin{tabular}{lrrrrrr}
\hline Case & $\zeta$ & $\nu$ & $x_{N}$ & $N$ & $\left|X^{\prime}\left(x_{N}\right)\right|$ & $\left|X^{\prime}\left(i \omega_{u}\right)\right|$ \\
\hline C1 & 0.50 & 0.35 & $-6.49+11.88 i$ & 27 & 0.3309 & 0.2025 \\
C2 & 0.50 & 0.20 & $-6.21+9.72 i$ & 18 & 0.1677 & 0.1212 \\
C3 & 0.50 & 0.05 & $-5.26+8.82 i$ & 10 & 0.0315 & 0.0296 \\
C4 & 2.00 & 0.35 & $-13.07+31.76 i$ & 193 & 0.8619 & 1.3188 \\
C5 & 2.00 & 0.08 & $-61.19+32.18 i$ & 2983 & 0.9907 & 0.3422 \\
C6 & 2.00 & 0.03 & $-43.76+0.00 i$ & 18 & 0.1678 & 0.1291 \\
C7 & 3.00 & 0.35 & $-13.48+39.86 i$ & 278 & 0.9025 & 1.9178 \\
C8 & 3.00 & 0.05 & $-99.15+43.96 i$ & 9800 & 0.9972 & 0.3083 \\
C9 & 3.00 & 0.02 & $-67.97+0.00 i$ & 18 & 0.1643 & 0.1236 \\
\hline
\end{tabular}

Table 2.2: Example 1: Approximated complex eigenvalues $x_{N}$ from sequence $x_{n}=$ $X\left(x_{n-1}\right)$ with initial point $x_{0}=i \omega_{u}=10 i$. $N$ number of iterations for error $\epsilon_{N}<10^{-12}$.

may converge to some non-viscous eigenvalue. For the current example, to reach this convergence the derivatives need to verify either $\left|X^{\prime}(\sigma)\right|<1$ or $\left|Y^{\prime}(\sigma)\right|<1$, where $\sigma \in \mathbb{R}$ is any non-viscous real eigenvalue. The analytical expression of these derivatives can be calculated, if $X(\sigma)=\sigma$ or $Y(\sigma)=\sigma$ then

$$
X^{\prime}(\sigma)=Y^{\prime}(\sigma)=\frac{\zeta \sigma \mu \omega_{u}}{(\sigma+\mu)\left[\sigma(\sigma+\mu)+\zeta \mu \omega_{u}\right]}
$$

For lightly damped systems, non-viscous eigenvalues $\sigma$ are located close to the pole $-\mu$ of the kernel function, [31]. Then, since the expression $\sigma+\mu \approx 0$ appears in the Eq. (2.59) denominator, it follows that the lighter the damping the higher the absolute value of the derivatives. For light damping, it is expected that the sequences $\left\{x_{n}\right\},\left\{y_{n}\right\}$ will not converge to real numbers. A high level of damping can facilitate the convergence, resulting in low derivative absolute values that may evenbe less than one. For the current example, the application of the method to obtain non-viscous eigenvalues gives results that are shown in Table 2.3; the same initial point has been taken for both sequences, i.e., $x_{0}=y_{0}$. The recursive results are considered successful if they converge to a real number with iterations that stay within the real domain, but it can be observed that for all cases the sequence $x_{n}=X\left(x_{n-1}\right)$ does not satisfactorily converge to non-viscous eigenvalues. For example, for cases $\mathrm{C} 1, \mathrm{C} 2, \mathrm{C} 3, \mathrm{C} 7$, the sequence $\left\{x_{n}\right\}$ does not stay in the real domain and finally converges to complex eigenvalues. Cases C6, C9 are over-damped and their sequences also escapes from the real numbers line before returning to converge to a real number. The sequences of cases $\mathrm{C} 4, \mathrm{C} 5, \mathrm{C} 8$ remain inside the real numbers but enter a non convergent infinite loop. 
The sequence $y_{n}=Y\left(y_{n-1}\right)$ produces quite different results. Convergence occurs for all high damping cases (C4-C9, in bold) towards non-viscous eigenvalues $\sigma$, verifying $y_{n} \in \mathbb{R}, \forall n \geq 0$. Therefore, the sequence reaches the real numbers remaining in the real axis and obviously $\left|Y^{\prime}(\sigma)\right|<1$. The rest of cases $\mathrm{C} 1, \mathrm{C} 2, \mathrm{C} 3$ converge to complex eigenvalues as with $x_{n}=X\left(x_{n-1}\right)$.

\begin{tabular}{|c|c|c|c|c|c|c|c|}
\hline \multirow[b]{2}{*}{ Case } & \multirow[b]{2}{*}{$x_{0}, y_{0}$} & \multicolumn{3}{|c|}{ Iteration sequence of $X(s)$} & \multicolumn{3}{|c|}{ Iteration sequence of $Y(s)$} \\
\hline & & $x_{N}$ & $N$ & $\left|X^{\prime}\left(x_{N}\right)\right|$ & $y_{N}$ & $N$ & $Y^{\prime}\left(y_{N}\right)$ \\
\hline $\mathrm{C} 1$ & -21.43 & $-6.49+11.88 i$ & 29 & 0.3309 & $-6.49-11.88 i$ & 28 & 0.3309 \\
\hline $\mathrm{C} 2$ & -37.50 & $-6.21+9.72 i$ & 26 & 0.1677 & $-6.21-9.72 i$ & 18 & 0.1677 \\
\hline C3 & -150.00 & $-5.26+8.82 i$ & 11 & 0.0315 & $-5.26-8.82 i$ & 11 & 0.0315 \\
\hline $\mathrm{C} 4$ & -14.29 & - & n.c. & 1.4452 & -2.422 & 15 & 0.1042 \\
\hline C5 & -62.50 & - & n.c. & 1.0456 & -2.615 & 10 & 0.0245 \\
\hline C6 & -166.67 & $-43.76+0.00 i$ & 18 & 0.1678 & -2.655 & 8 & 0.0092 \\
\hline $\mathrm{C} 7$ & -14.29 & $-13.48+39.86 i$ & 276 & 0.9025 & -1.613 & 12 & 0.0631 \\
\hline $\mathrm{C} 8$ & -100.00 & - & n.c. & 0.3078 & -1.700 & 8 & 0.0091 \\
\hline C9 & -250.00 & $-67.97+0.00 i$ & 18 & 0.1643 & -1.710 & 7 & 0.0036 \\
\hline
\end{tabular}

Table 2.3: Example 1: Results for non-viscous eigenvalues; cases converging to nonviscous eigenvalues emphasized in bold. $N$ number of iterations, $\left|X^{\prime}\left(x_{N}\right)\right|,\left|Y^{\prime}\left(y_{N}\right)\right|$ recursive function derivative evaluated in sequence limit. Non convergent sequences named "n.c."

In this example, it has been shown that the proposed method not only can be used to compute efficiently complex eigenvalues but also non-viscous ones. Furthermore, the convergence to the latter is achieved when the damping induced in the system is high. This duality is an important advantage, because the published methods that are oriented to the calculation non-viscous eigenvalues [31, 32] often assume light damping.

\subsubsection{Example 2: Multiple Degree-of-Freedom Systems}

To complete the validation of the numerical method, a four-dof discrete system with four masses and two types of viscoelastic links is studied, as shown in Fig. 2.3. Links $A$ relate internal forces with relative displacements and velocities of consecutive masses (each one an active dof) through a five-kernels Biot's model; links B directly constraint each mass through a four-parameter viscoelastic model based on the fractional derivatives [20]. Indexes $j=0,5$ of fixed boundaries are related with zero dof's so that $u_{0}=\dot{u}_{0}=u_{5}=\dot{u}_{5}=0$. Thus, the constitutive equations 


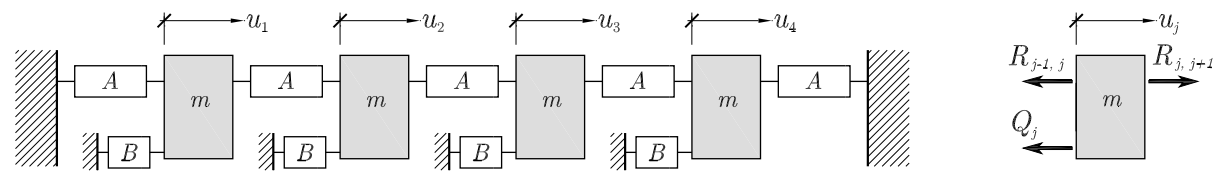

Figure 2.3: Example 2: Lumped-mass dynamical system with viscoelastic links. Links $A$ follows the Biot's model with five kernels, links $B$ based on the fractional derivatives.

relating reactions and displacements are

$\operatorname{Links} A, 1 \leq j \leq 5: \quad R_{j-1, j}=k_{a}\left(u_{j}-u_{j-1}\right)+\int_{-\infty}^{t} \mathcal{G}_{a}(t-\tau)\left(\dot{u}_{j}-\dot{u}_{j-1}\right) \mathrm{d} \tau$

$\operatorname{Links} B, 1 \leq j \leq 4: \quad Q_{j}+T_{r}^{\gamma} \frac{\mathrm{d}^{\gamma} Q_{j}}{\mathrm{~d} t^{\gamma}}=k_{b}\left(u_{j}+c T_{r}^{\gamma} \frac{\mathrm{d}^{\gamma} u_{j}}{\mathrm{~d} t^{\gamma}}\right)$

where $c, \gamma$ and $T_{r}$ are the parameters of the damping model based on the fractional derivatives and for real materials $c>1,0<\gamma<1, T_{r}>0$. The coefficients $k_{a}$, $k_{b}$ are the linear, static rigidities of links $A$ and $B$, respectively and the function

$$
\mathcal{G}_{a}(t)=\frac{c_{v}}{5} \sum_{l=1}^{5} \mu_{l} e^{-\mu_{l} t}
$$

is the kernel that controls the damping behavior of links $A$. The damping coefficient $c_{v}=2 m \omega_{a} \zeta$ is expressed as function of a certain damping ratio $\zeta$ and of the reference frequency, $\omega_{a}=\sqrt{k_{a} / m}$. Its Laplace transform is directly

$$
G_{a}(s)=\frac{c_{v}}{5} \sum_{l=1}^{5} \frac{\mu_{l}}{s+\mu_{l}}
$$

resulting for links $A$ the following frequency-dependent stiffness relation between internal forces and dof's

$$
\hat{R}_{j-1, j}(s)=\left[k_{a}+s G_{a}(s)\right]\left[\hat{u}_{j}(s)-\hat{u}_{j-1}(s)\right]
$$

where $\hat{R}_{j-1, j}(s)=\mathcal{L}\left\{R_{j-1, j}(t)\right\}$ and $\hat{u}_{j}(s)=\mathcal{L}\left\{u_{j}(t)\right\}$ are the Laplace transform of the internal forces and of the dof's, respectively.

For viscoelastic links $B$ based on fractional derivatives, an explicit expression of the kernel function $\mathcal{G}_{b}(t)$ is not analytically available. However, the damping function in the Laplace domain $G_{b}(s)$ can easily be calculated simply applying to the fractional derivatives of Eq. (2.60) the Laplace transform and using its 


\begin{tabular}{ccccccc} 
Mass $(\mathrm{kg})$ & \multicolumn{2}{c}{ Rigidities $(\mathrm{N} / \mathrm{m})$} & \multicolumn{4}{c}{ Natural frequencies $(\mathrm{rad} / \mathrm{s})$} \\
\hline$m$ & $k_{a}$ & $k_{b}$ & $\omega_{1}$ & $\omega_{2}$ & $\omega_{3}$ & $\omega_{4}$ \\
\hline $10^{3}$ & $10^{5}$ & $5 \times 10^{4}$ & 9.391 & 13.718 & 17.658 & 20.293 \\
\hline
\end{tabular}

Table 2.4: Example 2: Mass, static rigidities and modal natural frequencies for the 4-dof undamped system.

properties. If $\hat{Q}_{j}(s)=\mathcal{L}\left\{Q_{j}(t)\right\}$ is the Laplace transform of the reactions at links $B$, then

$$
\hat{Q}_{j}(s)=k_{b} \frac{1+c\left(T_{r} s\right)^{\gamma}}{1+\left(T_{r} s\right)^{\gamma}} \hat{u}_{j}(s) \equiv\left[k_{b}+s G_{b}(s)\right] \hat{u}_{j}(s)
$$

where

$$
G_{b}(s)=\frac{k_{b}}{s} \frac{(c-1)\left(T_{r} s\right)^{\gamma}}{1+\left(T_{r} s\right)^{\gamma}}
$$

The free-motion equations in the Laplace domain can be obtained assembling the mass and the stiffness matrices associated with the structural configuration shown in Fig. 2.3, resulting in an equilibrium similar to that of Eq. (2.2)

$$
\left[s^{2} \mathbf{M}+s \mathbf{G}(s)+\mathbf{K}\right] \hat{\mathbf{u}}(s)=\mathbf{0}
$$

where $\mathbf{M}=m \mathbf{I}_{4}, \mathbf{K}=k_{a} \boldsymbol{\Pi}_{a}+k_{b} \boldsymbol{\Pi}_{b}$ and $\mathbf{G}=G_{a}(s) \boldsymbol{\Pi}_{a}+G_{b}(s) \boldsymbol{\Pi}_{b}$, with auxiliary matrices

$$
\boldsymbol{\Pi}_{a}=\left[\begin{array}{rrrr}
2 & -1 & 0 & 0 \\
-1 & 2 & -1 & 0 \\
0 & -1 & 2 & -1 \\
0 & 0 & -1 & 2
\end{array}\right] \quad, \quad \Pi_{b}=\mathbf{I}_{4}
$$

Table 2.4 showns the values of mass $m$, static rigidities $k_{a}, k_{b}$ of the springs and natural frequencies of the undamped system. Notice that the damping matrix $\mathbf{G}(s)$ can be expressed as linear combination of the stiffness and mass matrices in the following form

$$
\mathbf{G}(s)=\frac{G_{a}(s)}{k_{a}} \mathbf{K}+\frac{k_{a} G_{b}(s)-k_{b} G_{a}(s)}{k_{a} m} \mathbf{M}
$$

Under these conditions, the viscoelastic damping matrix $\mathbf{G}(s)$ is said to be proportional, [29], and it can be assured that $\boldsymbol{\Gamma}(s)=\boldsymbol{\Phi}^{T} \mathbf{G}(s) \boldsymbol{\Phi}$ is diagonal. Thus, according to the theoretical results presented in Sec. 2.3 and after some operations, the non-dimensional function $J_{j}(s)$ is

$$
J_{j}(s)=\frac{\phi_{j}^{T} \mathbf{G}(s) \phi_{j}}{2 m_{j} \omega_{j}}=\frac{G_{a}(s)}{2 k_{a}} \omega_{j}+\frac{k_{a} G_{b}(s)-k_{b} G_{a}(s)}{2 k_{a} m \omega_{j}}
$$


where $\omega_{j}$ is the undamped frequency of the $j$ th mode. Consequently, the functions $X_{j}(s), Y_{j}(s)$ defined in Eqs. (2.47) can be constructed to compute the complex eigenvalues using the iterative process defined by the sequence of iterates

$$
x_{j}^{(n)}=X_{j}\left(x_{j}^{(n-1)}\right), \quad y_{j}^{(n)}=Y_{j}\left(y_{j}^{(n-1)}\right), \quad 1 \leq j \leq 4
$$

As shown in Theorem 3, the convergence speed depends on the damping level induced in the system by the viscoelastic model. For this reason, in the current example three separated cases corresponding to three different damping levels will be studied: lightly damped (LD), moderately damped (MD) and strongly damped (SD) systems. In order to numerically differentiate these levels, the loss factor peak will be used as damping index. As known [11, 12, 21, 22, 75], the loss factor of a frequency-domain constitutive equation is a real-valued and frequency-dependent function defined as the quotient between the imaginary and the real part of the complex stiffness. Here, the complex stiffness associated with the viscoelastic links of the structure shown in Fig. 2.3 are

$$
\begin{array}{llll}
\hat{k}_{a}(i \omega)= & k_{a}+i \omega G_{a}(i \omega)= & M_{a}(\omega)+i L_{a}(\omega)= & M_{a}(\omega)\left[1+i \eta_{a}(\omega)\right] \\
\hat{k}_{b}(i \omega)= & k_{b}+i \omega G_{b}(i \omega)= & M_{b}(\omega)+i L_{b}(\omega)= & M_{b}(\omega)\left[1+i \eta_{b}(\omega)\right]
\end{array}
$$

where $M_{a}(\omega), M_{b}(\omega)$ are the real part of the complex stiffness or dynamic modulus; $L_{a}(\omega), L_{b}(\omega)$ the imaginary part or loss modulus and finally $\eta_{a}(\omega)=L_{a}(\omega) / M_{a}(\omega)$ , $\eta_{b}(\omega)=L_{b}(\omega) / M_{b}(\omega)$ the loss factors. In general, for real solid materials, the loss factor presents one maximum peak [20]. The function takes at the peak a representative value of the induced level of damping $\eta_{m}$ named loss factor peak. For the current viscoelastic links, the loss factor peaks are defined as

$$
\eta_{m A}=\max _{\omega \geq 0} \eta_{a}(\omega), \quad \eta_{m B}=\max _{\omega \geq 0} \eta_{b}(\omega)
$$

Pritz [21] studied the frequency dependence of the loss factor for real solid materials. According to the experimental evidence, the reference gave the following classification by order of $\eta_{m}$ level:

- Stiff structural materials present light damping with low $0.001 \leq \eta_{m} \leq 0.01$

- Moderately damped materials, such as plastics, with medium $0.01 \leq \eta_{m} \leq$ 0.10

- Rubbers and rubber-like materials used for vibrations control, induce high damping level and consequently $0.10 \leq \eta_{m} \leq 1.0$

This classification is used in the current example to differentiate the three numerical cases. The relaxation parameters of the Biot's multiexponential model (links A) are $\mu_{l}=\{5,10,13,18,40\} \mathrm{rad} / \mathrm{s}$. For links $B$, the relaxation time is $T_{r}=10^{-3}$ $\mathrm{s}$ and the rest of parameters, say $\zeta, c, \gamma$ are chosen so that the computed loss 


\begin{tabular}{lcccccc} 
& \multicolumn{3}{c}{ Links type $A$} & & \multicolumn{3}{c}{ Links type $B$} \\
\cline { 2 - 3 } \cline { 6 - 7 } Cases & $\zeta$ & $\eta_{m A}$ & & $c$ & $\gamma$ & $\eta_{m B}$ \\
\hline Lightly Damped & 0.007 & $\mathbf{0 . 0 1 0 0}$ & & 1.135 & 0.20 & $\mathbf{0 . 0 1 0 0}$ \\
Moderately Damped & 0.078 & $\mathbf{0 . 1 0 0 1}$ & & 1.860 & 0.40 & $\mathbf{0 . 1 0 0 3}$ \\
Strongly Damped & 2.000 & $\mathbf{1 . 0 0 1 2}$ & & 94.00 & 0.60 & $\mathbf{1 . 0 0 9 1}$ \\
\hline
\end{tabular}

Table 2.5: Example 2: Numerical cases and computed loss factor peaks of the viscoelastic links for $\left\{\mu_{l}\right\}_{l=1}^{5}=\{5,10,13,18,40\} \mathrm{rad} / \mathrm{s}, T_{r}=10^{-3} \mathrm{~s}$.

factor peaks given by Eq. (2.71) take the values: LD is associated with $\eta_{m} \approx 0.01$, MD with $\eta_{m} \approx 0.10$ and SD with $\eta_{m} \approx 1.00$. Table 2.5 shows the parameters and the calculated loss factor peak values for each link type, which will be used in the following.

The results of the recursive scheme are presented in Table 2.6. For each mode, the initial value is the undamped eigenvalue $x_{j}^{(0)}=i \omega_{j}$. As before, the iteration sequence continues until the non-dimensional iterative error $\epsilon_{j}^{(n)}$ defined as in Eq. (2.58) is lower than the prefixed value $\epsilon_{\max }=10^{-12}$. The number of computed iterations $N$ shown in the last column increases with the level of damping and as predicted, lightly damped systems present faster convergence. In Fig. 2.4 the path traced by the elements of the sequence $\left\{x_{j}^{(n)}\right\}_{n=0}^{N}$ in the complex plane has been drawn for each of the four modes, top figures. In addition, the error versus iteration curves have been plotted, bottom figures. For LD, MD the recursive sequences in the complex plane do not present excessive numerical oscillations around the fixed point (square). However, the path for SD behaves similarly as those of the highly damped cases from Example 1. In this case, the first iterations fluctuate, and the error does not decay linearly, a characteristic of contractive functions. Only when the elements of the sequence are close enough to the fixed point, the rate of decay remains constant.

\subsection{Conclusions}

In this paper, a new numerical method to compute the eigenvalues of linear viscoelastic structures is developed. The method can be implemented for single or multiple degree of freedom systems with a proportional, or lightly nonproportional, damping matrix. Several transformations in the characteristic equation lead us to find two relevant complex functions to be used in a recursive scheme. 


\begin{tabular}{|c|c|c|c|c|}
\hline \multirow[b]{2}{*}{ Cases } & Mode & Initial value & $N$ th-Approx. & \# Iterat. \\
\hline & $j$ & $x_{j}^{(0)}$ & $x_{j}^{(N)}$ & $N$ \\
\hline \multirow[t]{4}{*}{ Lightly Damped } & 1 & $9.391 i$ & $-0.0395+9.5017 i$ & 7 \\
\hline & 2 & $13.718 i$ & $-0.0637+13.8311 i$ & 7 \\
\hline & 3 & $17.658 i$ & $-0.0865+17.7910 i$ & 7 \\
\hline & 4 & $20.292 i$ & $-0.1012+20.4453 i$ & 7 \\
\hline \multirow[t]{4}{*}{ Moderately Damped } & 1 & $9.391 i$ & $-0.3504+9.7831 i$ & 9 \\
\hline & 2 & $13.718 i$ & $-0.6568+14.3957 i$ & 10 \\
\hline & 3 & $17.658 i$ & $-0.9195+18.7015 i$ & 11 \\
\hline & 4 & $20.292 i$ & $-1.0804+21.6179 i$ & 11 \\
\hline \multirow[t]{4}{*}{ Strongly Damped } & 1 & $9.391 i$ & $-12.4924+25.1788 i$ & 41 \\
\hline & 2 & $13.718 i$ & $-13.6958+37.8174 i$ & 56 \\
\hline & 3 & $17.658 i$ & $-14.0601+49.3423 i$ & 68 \\
\hline & 4 & $20.292 i$ & $-14.1392+56.9869 i$ & 74 \\
\hline
\end{tabular}

Table 2.6: Example 2: Approximated eigenvalues $x_{N}$ after $N$ iterations. Sequence is considered to converge if relative error satisfies $\epsilon_{j}^{(N)}<10^{-12}$.

The main theoretical conclusions are presented in three theorems. With the help of fixed point theory, Theorem 1 demonstrates the existence of complex eigenvalues considered as fixed points of the recursive functions. Theorems 2 and 3 analyze the convergence of the recursive sequence by means of the Banach fixed point theorem. It is shown that the convergence speed of the proposed recursive method depends on the level of damping and on the viscoelasticity. It is proved that systems with low-damping together with low viscoelasticity present in general faster convergence. For strongly damped structures the iterative process is slower although the convergence is ensured.

To illustrate and validate the theoretical results, two numerical examples are analyzed. First, a single degree of freedom viscoelastic system with a Biot damping model is studied. Since the overdamped region is available, the level of damping and the speed of the numerical method can be related. In all the numerical cases considered, the recursive sequence has always converged, even when the system is overdamped. Moreover, this example shows that under certain conditions the method may also be used to obtain non-viscous eigenvalues. As predicted by the theory, the cases associated with low damping systems converged faster, and conversely, high damping systems converged slowly. Second, in order to study multiple degree of freedom systems, a four-mass discrete structure with proportional damping is examined. The damping is introduced through viscoelastic links using again Biot's model with five kernels and a damping model based on the fractional derivatives. With the objective to relate the level of damping with the convergence 

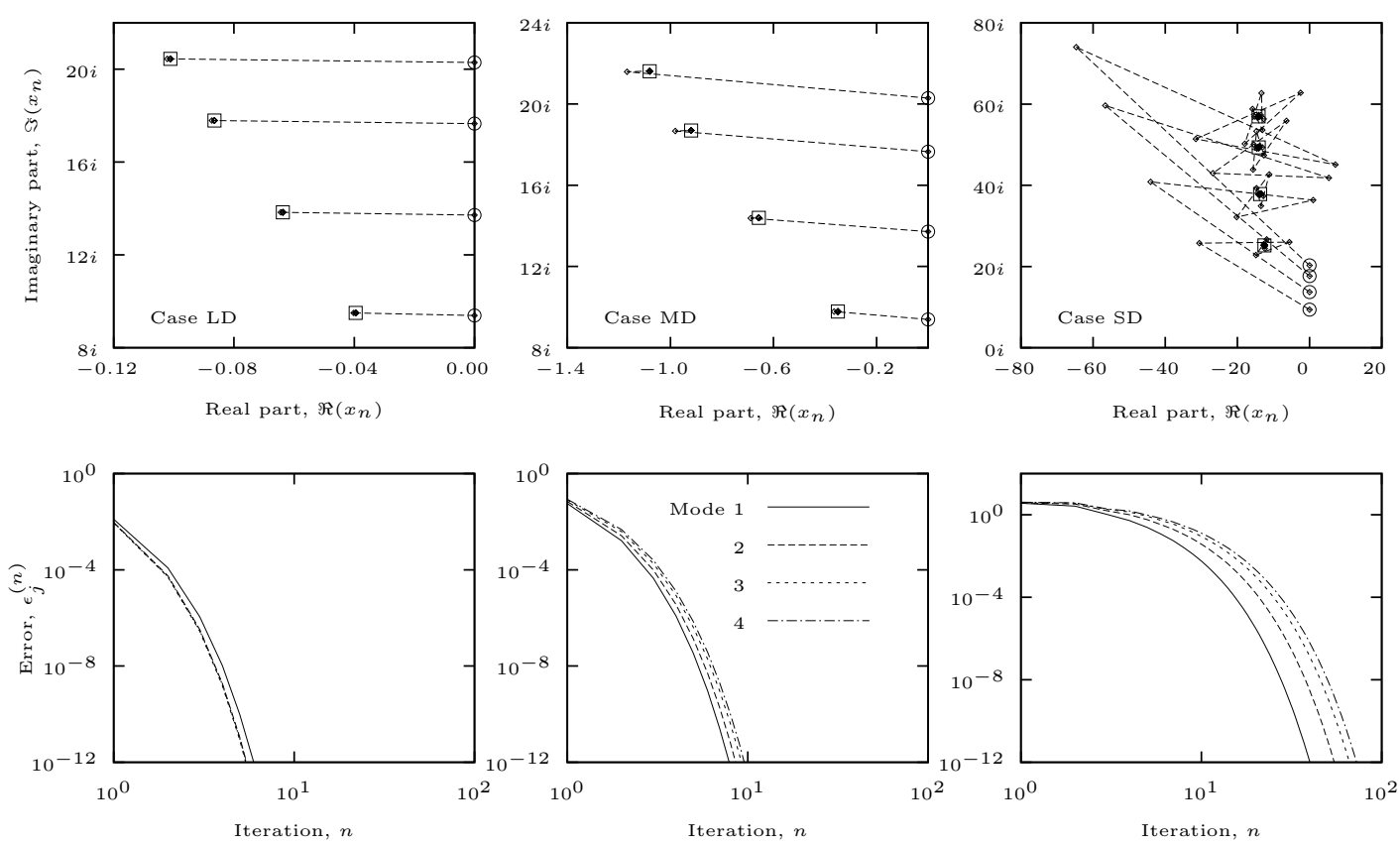

Figure 2.4: Example 2: Four mode paths in complex plane of iterates' sequence from initial point $\odot$ to limit eigenvalue $\odot$ for Lightly, Moderately and Strongly Damped, top figures. Associated error $\epsilon_{j}^{(n)}$, bottom figures.

three cases with different levels are studied. Further research is currently being developed by the authors for the generalization of the method for multiple degrees of freedom systems including a damping matrix whose non-proportionality cannot be neglected.

\section{Appendix 2.A. Proof of properties P1 to P5}

Proof of P1 The complex functions $X(s), Y(s)$ defined in Eqs. (2.10) can be considered a composition of analytical functions. Hence, $X=\omega_{u}(f \circ J), Y=$ $\omega_{u}(g \circ J)$, where

$$
f(z)=-z+i \sqrt{1-z^{2}}, \quad g(z)=-z-i \sqrt{1-z^{2}}
$$

are analytical functions for any $z \in \mathbb{C}$ except in the set $\{z \in \mathbb{R}:|z| \geq 1\}$. Therefore, from the functions' composition properties, $X(s), Y(s)$ are analytical in the set where $J(s)$ is, except in points of the region $\mathcal{B}=\{z \in \mathbb{R}:|J(z)| \geq 1\}$ where 
the square root is not analytical.

Proof of P2 Since $X(s), Y(s)$ are analytical and consequently holomorphic in the set $\mathcal{A}$, then

$$
X^{*}(s)=\omega_{u}\left[-J(s)+i \sqrt{1-J^{2}(s)}\right]^{*}=\omega_{u}\left[-J\left(s^{*}\right)+(-i) \sqrt{1-J^{2}\left(s^{*}\right)}\right]=Y\left(s^{*}\right)
$$

Proof of P3 Let $z \in \mathcal{A}$ be a fixed point of $X(s)$, then $z=X(z)$. Taking the complex conjugate of both terms and using P2, it follows that $z^{*}=X^{*}(z)=Y\left(z^{*}\right)$, from where $z^{*}$ is a fixed point of $Y(s)$. It is clear that using the same principle the reciprocal also holds.

Proof of P4 From the hypothesis H3, $J(s) \in \mathcal{C}, \forall s \in \mathcal{C}$. Due to $X=\omega_{u}(f \circ J)$, it follows that $X(\mathcal{C}) \subset \mathcal{C}_{+}$holds only if $f(\mathcal{C}) \subset \mathcal{C}_{+}$. Taking a complex number $z=x+i y \in \mathcal{C}$, it is necessary to prove that $\Im\{f(z)\}>0$. By the definition of the imaginary part of a complex number

$$
\begin{aligned}
\Im\{f(z)\} & =\frac{f(z)-f^{*}(z)}{2 i}=\frac{1}{2 i}\left(-z+i \sqrt{1-z^{2}}+z^{*}-(-i) \sqrt{1-z^{* 2}}\right) \\
& =-\frac{z-z^{*}}{2 i}+\frac{\sqrt{1-z^{2}}+\sqrt{1-z^{* 2}}}{2 i}=-\Im\{z\}+\Re\left\{\sqrt{1-z^{2}}\right\}(2.7
\end{aligned}
$$

Let now $u+i v \in \mathbb{C}$ be the main square root of $1-z^{2}$, then $(u+i v)^{2}=1-z^{2}=$ $1-(x+i y)^{2}$ where $u=\Re\left\{\sqrt{1-z^{2}}\right\} \geq 0$. Let us calculate $u$ by identification of real and imaginary parts in the previous equality

$$
u^{2}-v^{2}=1-x^{2}+y^{2}, \quad u v=-x y
$$

From the expression, an equation in only the variable $u$ may be deduced. Thus, the real part $u$ may be computed as a root of the four-order polynomial $u^{4}-$ $\rho u^{2}-(x y)^{2}=0$, where $\rho=1-x^{2}+y^{2}$. Therefore, cases $x=0, x \neq 0$ must be considered separately.

If $x=0$ then $\rho=1+y^{2}$ and the polynomial equation leads to $u^{2}\left(u^{2}-1-y^{2}\right)=0$, hence, either $u=0$ or $u^{2}=1+y^{2}$. For the first, it is verified from Eq. (2.75) that $-v^{2}=1+y^{2}>0$, a contradiction; consequently $u^{2}=1+y^{2}$. The negative solution is not of interest since $u$ is defined by a main square root. Therefore

$$
u=\Re\left\{\sqrt{1-z^{2}}\right\}=\sqrt{1+y^{2}}>y=\Im\{z\} \Rightarrow \Im\{f(z)\}>0 .
$$

If $x \neq 0$, the solutions of the four-order polynomial can be expressed in the form $u^{2}=\left(\rho \pm \sqrt{\rho^{2}+4(x y)^{2}}\right) / 2$. Since $u^{2}>0$, the solution $\rho-\sqrt{\rho^{2}+4(x y)^{2}}<0$ 
must be rejected. Now, let us suppose that $u=\Re\left\{\sqrt{1-z^{2}}\right\} \leq y=\Im\{z\}$ in order to arrive to a contradiction

$$
\begin{aligned}
0 \leq u \leq y \Rightarrow 2 u^{2} \leq 2 y^{2} \Rightarrow \rho+\sqrt{\rho^{2}+4(x y)^{2}} \leq 2 y^{2} & \Rightarrow \\
\sqrt{\rho^{2}+4(x y)^{2}} \leq 2 y^{2}-\rho \Rightarrow \rho^{2}+4(x y)^{2} \leq 4 y^{2}-4 y^{2} \rho+\rho^{2} & \Rightarrow \\
x^{2} \leq y^{2}-\rho=y^{2}-1+x^{2}-y^{2} & \Rightarrow 0 \leq(2.77)
\end{aligned}
$$

The last inequality is the contradiction, for this reason necessarily $\Im\{f(z)\}=$ $u-y>0$ also for $x \neq 0$. Therefore, $\Im\{X(s)\}>0, \forall s \in \mathcal{C}$ that is equivalent to $X(\mathcal{C}) \subset \mathcal{C}_{+}$.

Now, if $s \in \mathcal{C}$, then $X\left(s^{*}\right) \in \mathcal{C}_{+}$and $\Im\left\{X\left(s^{*}\right)\right\}>0$. Therefore $\Im\left\{X^{*}\left(s^{*}\right)\right\}=$ $\Im\{Y(s)\}<0$ that proves the property for the function $Y(s)$.

Proof of P5 Let us consider an element $x \in \mathcal{B} \subset \mathbb{R}$. From the definition of the set $\mathcal{B},|J(x)| \geq 1$ is verified and from the main hypothesis of the propery $J(\mathbb{R}) \subset \mathbb{R}$, therefore, $z=J(x) \in \mathbb{R}$. Thus, the number $1-z^{2} \leq 0$ and $\sqrt{1-z^{2}}=i \sqrt{\left|1-z^{2}\right|}$ is pure imaginary. Hence, the value of the function is $f(z)=-z-\sqrt{\left|1-z^{2}\right|} \in \mathbb{R}$ so that $X(\mathcal{B}) \subset \mathbb{R}$.

Now, let us consider a number $x \in \mathbb{R} \backslash \mathcal{B}$ and by definition $|J(x)|<1$ and $1-z^{2}>0$. Consequently, $\Im\{f(z)\}=\sqrt{1-z^{2}}>0$ whence $\Im\{X(x)\}>0$ or, in set inclusion notation, $X(\mathbb{R} \backslash \mathcal{B}) \subset \mathcal{C}_{+}$. For statements $Y(\mathcal{B}) \subset \mathbb{R}$ and $Y(\mathbb{R} \backslash \mathcal{B}) \subset \mathcal{C}_{-}$, the same procedure as that of $X(s)$ can be used. 


\section{Bibliography}

[7] M.A. Biot. "Variational Principles in Irreversible Thermodynamics with Application to Viscoelasticity." In: Physical Review 97.6 (1955), 1463-1469 (cit. on pp. 3, 20, 24, 37, 58, 82, 93, 192).

[11] A.D. Nashif, D.I.G. Jones, and J.P. Henderson. Vibration Damping. John Wiley and Sons, 1985 (cit. on pp. 3, 45, 160, 192, 194, 206).

[12] David I.G. Jones. Handbook of viscoelastic vibration damping. John Wiley \& Sons, 2001 (cit. on pp. 3, 45, 160, 192, 194).

[14] D.F. Golla and P.C. Hughes. "Dynamics of Viscoelastic Structures - A Time-domain, Finite-element Formulation." In: Journal of Applied MechanicsTransactions of the ASME 52.4 (1985), 897-906 (cit. on pp. 3, 20, 22, 24, $58,60,82,84,118,160,162,164,192)$.

[15] R.L. Bagley and P.J. Torvik. "A Theoretical Basis for the Application of Fractional Calculus to Viscoelasticity." In: Journal of Rheology 27.3 (1983), 201-210 (cit. on pp. 4, 20, 82, 192, 196).

[16] R.L. Bagley and P.J. Torvik. "Fractional Calculus - A Different Approach to the Analysis of Viscoelastically Damped Structures." In: AIAA Journal 21.5 (1983), 741-748 (cit. on pp. 4, 20, 24, 82, 192, 193, 196).

[20] T. Pritz. "Analysis of four-parameter fractional derivative model of real solid materials." In: Journal of Sound and Vibration 195.1 (1996), 103-115 (cit. on pp. 4, 42, 45, 150, 192, 196, 206).

[21] T. Pritz. "Frequency dependences of complex moduli and complex Posson's ratio of real solid materials." In: Journal of Sound and Vibration 214.1 (1998), 83-104 (cit. on pp. 4, 45, 150, 206).

[22] T Pritz. "Loss factor peak of viscoelastic materials: Magnitude to width relations." In: Journal of Sound and Vibration 246.2 (2001), 265-280 (cit. on pp. $4,45,150,196,206)$.

[26] Yuriy A. Rossikhin and Marina V. Shitikova. "Application of Fractional Calculus for Dynamic Problems of Solid Mechanics: Novel Trends and Recent Results." In: Applied Mechanics Reviews 63.1 (2010), 010801(1)010801(52) (cit. on pp. 4, 20). 
[28] S. Adhikari. "Dynamics of Non-viscously Damped Linear Systems." In: Journal of Engineering Mechanics 128.3 (2002), 328-339 (cit. on pp. 4-6, $22,35,36,58,84,94,121,125,129,131,160-162,165,168,170,171,203)$.

[29] S. Adhikari. "Classical normal modes in non-viscously damped linear systems." In: AIAA Journal 39.5 (2001), pp. 978-980 (cit. on pp. 5, 35, 44, $63,84,121)$.

[30] J. Woodhouse. "Linear Damping Models For Structural Vibration." In: Journal of Sound and Vibration 215.3 (1998), 547-569 (cit. on pp. 5, 35, $58,121,170,171)$.

[31] Sondipon Adhikari and Blanca Pascual. "Eigenvalues of linear viscoelastic systems." In: Journal of Sound and Vibration 325.4-5 (2009), 1000-1011 (cit. on pp. 5, 21, 35, 41, 42, 69, 70, 76, 83, 119, 120, 141, 151, 152, 193).

[32] Sondipon Adhikari and Blanca Pascual. "Iterative Methods for Eigenvalues of Viscoelastic Systems." In: Journal of Vibration and Acoustics 133.2 (2011), pp. 021002.1-021002.7 (cit. on pp. 5, 21, 35, 42, 59, 83, 108, 119, $171,193)$.

[34] M. Lázaro and J. L. Pérez-Aparicio. "Multiparametric computation of eigenvalues for linear viscoelastic structures." In: Computers \& Structures 117.0 (2013), pp. $67-81$ (cit. on pp. 10, 21, 194, 201).

[35] SS Ray and RK Bera. "Analytical solution of the Bagley Torvik equation by Adomian decomposition method." In: Applied Mathematics and Computation 168.1 (2005), 398-410 (cit. on p. 20).

[36] S. Saha Ray, K. S. Chaudhuri, and R. K. Bera. "Analytical approximate solution of nonlinear dynamic system containing fractional derivative by modified decomposition method." In: Applied Mathematics and Computation 182.1 (2006), 544-552 (cit. on p. 20).

[37] S. Saha Ray. "On Haar wavelet operational matrix of general order and its application for the numerical solution of fractional Bagley Torvik equation." In: Applied Mathematics and Computation 218.9 (2012), 5239-5248 (cit. on p. 20).

[38] K. J. Buhariwala. "Dynamic Response of Viscoelastic Fibre Reinforced Composite Materials." MA thesis. Institute for Aerospace Studies. University of Toronto., 1982 (cit. on pp. 20, 24).

[39] K.J. Buhariwala and J.S. Hansen. "Dynamics of Viscoelastic Structures." In: AIAA Journal 26.2 (1988), 220-227 (cit. on pp. 20, 24).

[40] D.J. McTavish and P.C. Hughes. "Modeling of Linear Viscoelastic Space Structures." In: Journal of Vibration and Acoustics-Transactions of the ASME 115.1 (1993), 103-110 (cit. on pp. 20, 24, 58, 82, 118, 160, 164). 
[41] GA Lesieutre and DL Mingori. "Finite-Element Modeling of Frequencydependent Material Damping Using Augmenting Thermodynamic Fields." In: Journal of Guidance Control and Dynamics 13.6 (1990), 1040-1050 (cit. on pp. $20,24,58,82,119)$.

[42] S. Adhikari. "Energy Dissipation in Vibrating Structures." MA thesis. Cambridge University Engineering Department, 1998 (cit. on pp. 20, 24).

[43] S. Adhikari and J. Woodhouse. "Identification Of Damping: PART 2, NonViscous Damping." In: Journal of Sound and Vibration 243.1 (2001), 63-88 (cit. on pp. 20, 24, 82).

[44] Mohamed Medjden and Nasser eddine Tatar. "Asymptotic behavior for a viscoelastic problem with not necessarily decreasing kernel." In: Applied Mathematics and Computation 167.2 (2005), pp. 1221 -1235 (cit. on p. 21).

[45] Nasser-eddine Tatar. "Uniform decay in viscoelasticity for kernels with small non-decreasingness zones." In: Applied Mathematics and Computation 218.15 (2012), 7939-7946 (cit. on p. 21).

[46] Wei H. Yang. "A method for eigenvalues of sparse $\lambda$-matrices." In: International Journal for Numerical Methods in Engineering 19.6 (1983), pp. 943948 (cit. on pp. 21, 83, 118, 193).

[47] KV Singh and YM Ram. "Transcendental eigenvalue problem and its applications." In: AIAA Journal 40.7 (2002), 1402-1407 (cit. on pp. 21, 83, 118).

[48] FW Williams and D Kennedy. "Reliable Use of Determinants to Solve Non-linear Structural Eigenvalue Problems Efficiently." In: International Journal for Numerical Methods in Engineering 26.8 (1988), 1825-1841 (cit. on pp. $21,83,118)$.

[49] E. M. Daya and M. Potier-Ferry. "A numerical method for nonlinear eigenvalue problems application to vibrations of viscoelastic structures." In: Computers 85 Structures 79.5 (2001), pp. 533 -541 (cit. on pp. 21, 83, 119).

[50] Laetitia Duigou, El Mostafa Daya, and Michel Potier-Ferry. "Iterative algorithms for non-linear eigenvalue problems. Application to vibrations of viscoelastic shells." In: Computer Methods in Applied Mechanics and Engineering 192.11-12 (2003), pp. 1323 -1335 (cit. on pp. 21, 119).

[51] H. Voss. "An Arnoldi Method for Nonlinear Eigenvalue Problems." In: BIT Numerical Mathematics 44 (2 2004), pp. 387-401 (cit. on pp. 21, 83, 118).

[52] H. Voss. "A Jacobi-Davidson method for nonlinear and nonsymmetric eigenproblems." In: Computers \& Structures 85.17-18 (2007), 1284-1292 (cit. on pp. $21,83,118)$. 
[53] MR Abdel-Aziz and SM El-Sayed. "Sensitivity analysis of the largest dependent eigenvalue functions of eigensystems." In: Applied Mathematics and Computation 100.2-3 (1999), 103-110 (cit. on p. 21).

[54] A Muravyov and SG Hutton. "Closed-form solutions and the eigenvalue problem for vibration of discrete viscoelastic systems." In: Journal of Applied Mechanics-Transactions of the ASME 64.3 (1997), 684-691 (cit. on pp. 21, 58, 83, 119, 160, 164, 193).

[55] A. Muravyov. "Forced vibration responses of viscoelastic structure." In: Journal of Sound and Vibration 218.5 (1998), 892-907 (cit. on pp. 21, 38, $83,119,160)$.

[56] S. Menon and J. Tang. "A state-space approach for the dynamic analysis of viscoelastic systems." In: Computers \& Structures 82.15-16 (2004), pp. 1123 -1130 (cit. on pp. 21, 83, 119, 160, 164, 193).

[57] M.I. Friswell, S. Adhikari, and Y. Lei. "Vibration analysis of beams with non-local foundations using the finite element method." In: International Journal for Numerical Methods in Engineering 71 (2007), 1365-1386 (cit. on p. 21).

[58] Sondipon Adhikari and Michael I. Friswell. "Eigenderivative analysis of asymmetric non-conservative systems." In: International Journal for $\mathrm{Nu}$ merical Methods in Engineering 51.6 (2001), pp. 709-733 (cit. on pp. 21, 119).

[59] S. Adhikari. "Derivative of Eigensolutions of Nonviscously Damped Linear Systems." In: AIAA Journal 40.10 (2002), 2061-2069 (cit. on pp. 21, 83, $119,126,132)$.

[60] F. Cortés and Maria Jesús Elejabarrieta. "Computational methods for complex eigenproblems in finite element analysis of structural systems with viscoelastic damping treatments." In: Computer Methods Appl. Mech. Engineering 195.44-47 (2006), 6448-6462 (cit. on pp. 21, 83, 119).

[61] F. Cortés and Maria Jesús Elejabarrieta. "An approximate numerical method for the complex eigenproblem in systems characterised by a structural damping matrix." In: Journal of Sound and Vibration 296.1-2 (2006), 166182 (cit. on pp. 21, 83, 119).

[62] M. Martinez-Agirre and M. J. Elejabarrieta. "Higher order eigensensitivitiesbased numerical method for the harmonic analysis of viscoelastically damped structures." In: International Journal for Numerical Methods in Engineering 88.12 (2011), 1280-1296 (cit. on p. 21).

[63] M. Lázaro and J. L. Pérez-Aparicio. "Parametric solutions of the eigenvalue problem for single degree-of-freedom viscoelastic systems." In: AIAA Journal. 0 (2013), pp. i-xvii (cit. on pp. 21, 59, 69, 70, 72, 75, 76, 193, 201). 
[64] P Muller. "Are the eigensolutions of a l-d.o.f. system with viscoelastic damping oscillatory or not?" In: Journal of Sound and Vibration 285.12 (2005), 501-509 (cit. on pp. 22, 38, 58, 84, 121, 162).

[65] K. Goebel, T. Sekowski, and A. Stachura. "Uniform Convexity of the Hyperbolic Metric and Fixed Point of Holomorphic Mappings in the Hilbert Ball." In: Nonlinear Analysis: Theory, Methods \& Applications 4.5 (1980), pp. 1011-1021 (cit. on pp. 25, 26).

[66] S. Reich. "Approximating fixed points of holomorphic mappings." In: Math. Japon. 37 (1992), pp. 457-459 (cit. on p. 26).

[67] S. Banach. "Sur les opérations dans les ensembles abstraits et leurs applications aux équations intégrales." In: Fundamenta Mathematicae 3 (1922), pp. 133-181 (cit. on p. 29).

[68] William A. Kirk and Brailey Sims. Handbook of Metric Fixed Point Theory. Ed. by William A. Kirk and Brailey Sims. Kluwer Academic Publishers, 2001 (cit. on pp. 29, 30).

[69] Robert M. McLeod. "Mean Value Theorems for Vector Valued Functions." In: Proceedings of the Edinburgh Mathematical Society (Series 2) 14.03 (1965), pp. 197-209 (cit. on pp. 29, 30).

[70] A. M. Ostrowski. Solutions of Equations in Euclidean and Banach Spaces. Ed. by Paul A. Smith and Samuel Ellenberg. Academic Press, 1973 (cit. on pp. 30, 34).

[71] S. Adhikari and J. Woodhouse. "Quantification of non-viscous damping in discrete linear systems." In: Journal of Sound and Vibration 260.3 (2003), pp. 499-518 (cit. on pp. 33, 88, 94, 125, 197, 203).

[72] E. Schroeder. "Über unedlich viele Algorithmen zur Auflösung der Gleichungen." In: Mathematische Annalen 2 (1870), pp. 317-365 (cit. on p. 34).

[73] A Muravyov and SG Hutton. "Free vibration response characteristics of a simple elasto-hereditary system." In: Journal of Vibration and AcousticsTransactions of the ASME 120.2 (1998), 628-632 (cit. on pp. 38, 58, 160, 162).

[74] S Adhikari. "Qualitative dynamic characteristics of a non-viscously damped oscillator." In: Proceedings of the Royal Society A-Mathematical Physical and Engineering Sciences 461.2059 (2005), 2269-2288 (cit. on pp. 38, 58, $84,121)$.

[75] JJ de Espindola, JMD Neto, and EMO Lopes. "A generalised fractional derivative approach to viscoelastic material properties measurement." In: Applied Mathematics and Computation 164.2 (2005), 493-506 (cit. on p. 45). 



\section{Characterization of Real Eigenvalues in Linear Viscoelastic Oscillators and the Non-viscous \\ Set}

\subsection{Introduction}

The dissipative forces of any single degree-of-freedom (dof) viscoelastic oscillator depend on the time history of the velocity via the convolution integral with a kernel function. In Fig. 3.1, $m$ is the dof related mass with response $u(t), k$ the linear stiffness and $F(t)$ the applied external force. The dynamic equilibrium of forces directly produces the motion equation, expressed as

$$
m \ddot{u}+\mathcal{F}_{d}(t)+k u=F(t)
$$

where

$$
\mathcal{F}_{d}(t)=\int_{-\infty}^{t} \mathcal{G}(t-\tau) \dot{u}(\tau) \mathrm{d} \tau
$$

is the internal dissipative forces. The hereditary function $\mathcal{G}(t)$ is named damping function in time domain, whereas its Laplace transform $G(s)=\mathcal{L}\{\mathcal{G}(t)\}$ is 


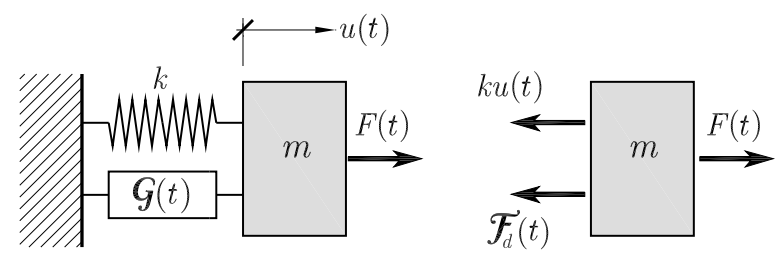

Figure 3.1: Single degree-of-freedom oscillator with a viscoelastic damper and associated free-body diagram.

the damping function in frequency domain. These functions control the damping mechanisms in a more general way than those of the viscous model, the simplest, classical and most used model of damping. In contrast, the mathematical complexity and storage requirements of the problem significantly increase: Eqs. (3.1), (3.2) lead to an integro-differential equation to be solved in $u(t)$. In order to obtain analytical solutions, a key issue is to know the set of eigenvalues of the homogeneous problem, $F(t) \equiv 0$. Testing then solutions with the form $u(t)=u_{0} e^{s t}$, Eq. (3.1) is transformed into the following characteristic equation

$$
m s^{2}+s G(s)+k=0
$$

It is well known that the number of roots of the previous equation is $2+N$ and that they can be noted as $\left\{\lambda, \lambda^{*}, \sigma_{1}, \ldots, \sigma_{N}\right\}$, where $\lambda, \lambda^{*}$ is a complex conjugate pair and $\sigma_{1}, \ldots, \sigma_{N}$ are $N$ negative real numbers named non-viscous eigenvalues The name is chosen precisely because they are characteristic of non-viscous or viscoelastic models. The number of these non-viscous eigenvalues will depend on the nature of the damping function, particularly on the number of hereditary exponential kernels. The complex conjugate pair forces the solution to be oscillatory, whereas the other eigenvalues are associated with overdamped, non-oscillatory modes. The latter modes decay rapidly and in general are not important for the system response. Perhaps this is the reason for which references focused on their theoretical study are rarely found in the bibliography.

The Biot's multiexponential model [7] possibly was the first to provide theoretical fundamentals based with variational principles to the viscoelastic behavior of materials. Other works have later been published based on this principle, mostly for its implementation in the finite element method [14, 40, 41]. Woodhouse [30], obtained approximated solutions of the transfer function for linear viscoelastic structures based on the residue-pole expansion. This work has been complemented by Adhikari [28] extending the modal analysis to general non-viscously damped systems, including the effect of the non-viscous modes. Muravyov [54] obtained analytical solutions in the time domain taking into account the non-viscous modes and using operational calculus. Adhikari [74, 76], Muravyov [73] and Muller [64] analyzed and discussed the problem of a single dof viscoelastic oscillator with a single 
exponential kernel damping. They also established the overdamped region, i.e., the set of parameters that induce an overdamped motion without complex eigenvalues. Adhikari and Pascual [77] developed an approximated method to obtain both complex and non-viscous eigenvalues for proportionally damped structures and based onto expanding the characteristic equation. In [32], the same authors improved the method introducing an iterative process. Lázaro and Pérez-Aparicio [63] obtained closed-form expressions of the non-viscous eigenvalues considering the eigenvalues as functions of the damping parameters. Also, they developed in [33] an efficient method based on the fixed-point iteration; under certain conditions, this scheme can also be applied to extract the non-viscous eigenvalues.

In the present paper, a mathematical characterization of the real eigenvalues related to a linear viscoelastic oscillator is presented. From this characterization, the existence of a set containing all real eigenvalues is derived, see Section 3.2. If the damping is governed by Biot's model with $N$ exponential kernels, this set -the non-viscous set- is formed by the union of $N$ closed intervals. Analytical and general solutions of the limits for these closed intervals are obtained for cases $N=1$ and $N=2$ in Section 3.2.1. In addition and only for light damping, in Section 3.2.2 approximated solutions for the general case of $N$ kernels are developed. Another contribution, Section 3.3, is the use of the non-viscous set to obtain a closed-form expression that estimates each non-viscous eigenvalue. The method is based on a quadratic interpolation of the characteristic equation in an interval of the non-viscous set using the proposed approximated limits. Finally, the two proposed contributions are validated with a numerical example in Section 3.4. The results of the non-viscous eigenvalues approximation are compared with other existing approaches from the bibliography.

\subsection{Location of real eigenvalues; the non-viscous set}

The present section is focused on giving a mathematical characterization for any real eigenvalue belonging to a single dof viscoelastic system. For that, Eq. (3.3) is rewritten in terms of the undamped natural frequency $\omega_{n}=\sqrt{k / m}$ and of the new dimensionless damping function defined as

$$
J(s)=\frac{G(s)}{2 m \omega_{n}}
$$

With the new variables, the characteristic Eq. (3.3) becomes

$$
s^{2}+2 s J(s) \omega_{n}+\omega_{n}^{2}=0
$$


Let us name $\sigma \in \mathbb{R}$ to any real eigenvalue of Eq. (3.5). We assume that the left hand side evaluated in $s=\sigma$ can be expressed in the following form

$$
\begin{aligned}
\sigma^{2}+2 \sigma J(\sigma) \omega_{n}+\omega_{n}^{2} & =[\sigma+A(\sigma)]^{2}+B(\sigma) \\
& =\sigma^{2}+2 \sigma A(\sigma)+A^{2}(\sigma)+B(\sigma)
\end{aligned}
$$

where $A(\sigma), B(\sigma)$ are two unknowns functions of $\sigma$ to determine. Obviously, infinite solutions exist for these unknowns, but those obtained by identifying the three coefficients of Eq. (3.6) to 1, $\sigma$ and $\sigma^{2}$ present important properties for the current purposes. It can easily verified that in such case

$$
A(\sigma)=J(\sigma) \omega_{n}, \quad B(\sigma)=\left[1-J^{2}(\sigma)\right] \omega_{n}^{2}
$$

and the characteristic equation is

$$
\left[\sigma+J(\sigma) \omega_{n}\right]^{2}+\left[1-J^{2}(\sigma)\right] \omega_{n}^{2}=0
$$

Assuming that the damping function is real-valued in the real domain, i.e. $J(\mathbb{R}) \subset$ $\mathbb{R}$, then $\sigma+J(\sigma) \omega_{n} \in \mathbb{R}$. Thus, rearranging the above equation it can be deduced that

$$
1-J^{2}(\sigma)=-\left[\frac{\sigma}{\omega_{n}}+J(\sigma)\right]^{2} \leq 0
$$

equivalent to $|J(\sigma)| \geq 1$. Note that this inequality is verified for any real eigenvalue and constitutes a mathematical characterization that can be resumed in the proposition: If $\sigma$ is a real eigenvalue of Eq. (3.5), then $|J(\sigma)| \geq 1$. As a direct consequence, defining the following set

$$
\mathcal{B}=\left\{s \in \mathbb{R}^{-}:|G(s)| \geq 2 m \omega_{n}\right\}
$$

assures that every real eigenvalue lies inside $\mathcal{B}$. This conclusion is valid even if the system is overdamped, with $2+N$ real eigenvalues all of them contained in $\mathcal{B}$. However, these systems are not of interest for the present paper and it will be considered that every real eigenvalue is non-viscous. Consequently, it is reasonable to assign the name non-viscous set for $\mathcal{B}$. Notice that $\mathcal{B}$ is defined as a subset of the negative real numbers because positive eigenvalues and dissipative behavior can not coexist.

The theoretical results presented have been obtained for any form of the damping function: No specification has been imposed on $G(s)$ except the necessary conditions given by Golla and Hughes [14] to define a strictly dissipative motion. Hereinafter, it will be assumed that the viscoelastic damping obeys a Biot's model with $N$ exponential kernels, restriction commonly assumed in engineering applications. The expression of the normalized damping functions in time and in frequency domain are respectively

$$
\mathcal{G}(t)=\frac{c_{v}}{N} \sum_{j=1}^{N} \mu_{j} e^{-\mu_{j} t}, \quad G(s)=\frac{c_{v}}{N} \sum_{j=1}^{N} \frac{\mu_{j}}{s+\mu_{j}}
$$


where $\mu_{j}>0$ with $1 \leq j \leq N$ are the relaxation or non-viscous parameters, and $c_{v}=\lim _{\mu_{j} \rightarrow \infty} G(s)$ the damping coefficient of the limit viscous model when the relaxation parameters become large. The damping function is normalized so that the integral over the whole time history is

$$
c_{v}=\int_{0}^{\infty} \mathcal{G}(t) d t
$$

It is common practice to use a damping ratio $\zeta=c_{v} / 2 m \omega_{n}$ instead of $c_{v}$, then the function $J(s)$ adopts the form

$$
J(s)=\frac{\zeta}{N} \sum_{j=1}^{N} \frac{\mu_{j}}{s+\mu_{j}}
$$

so that the set $\mathcal{B}$ is function only of the damping parameters. The following questions arise now

Q1. Under what conditions are analytical expressions for $\mathcal{B}$ available?

Q2. May approximated expressions for $\mathcal{B}$ always be obtained?

Q3. May the information provided by $\mathcal{B}$ be used for the numerical computation of non-viscous eigenvalues?

The rest of the paper aims to answer these questions. Previously, some remarks for the general structure of $\mathcal{B}$ are introduced with the help of an example. Choosing the relaxation parameters $\mu_{j}=\{3,5,8,12\} \mathrm{rad} / \mathrm{s}$, the function $J(s)$ is represented in Figs. 3.2 for different values of the damping ratio: Left figure $\zeta=0.2$ and right $\zeta=0.5$, sufficiently separated for the example purposes. From the definition from Eq. (3.10), the negative values $s$ that verify $J(s) \geq+1$ or $J(s)<-1$ lie inside $\mathcal{B}$. The different zones are defined by the intersection points between $J(s)$ and the horizontal lines with ordinates +1 and -1 . As shown in the figure, these zones correspond to four closed intervals around the four poles, and the definition of the intervals can be generalized for any number $N$. Indeed, for any pole $-\mu_{j}$ it is 

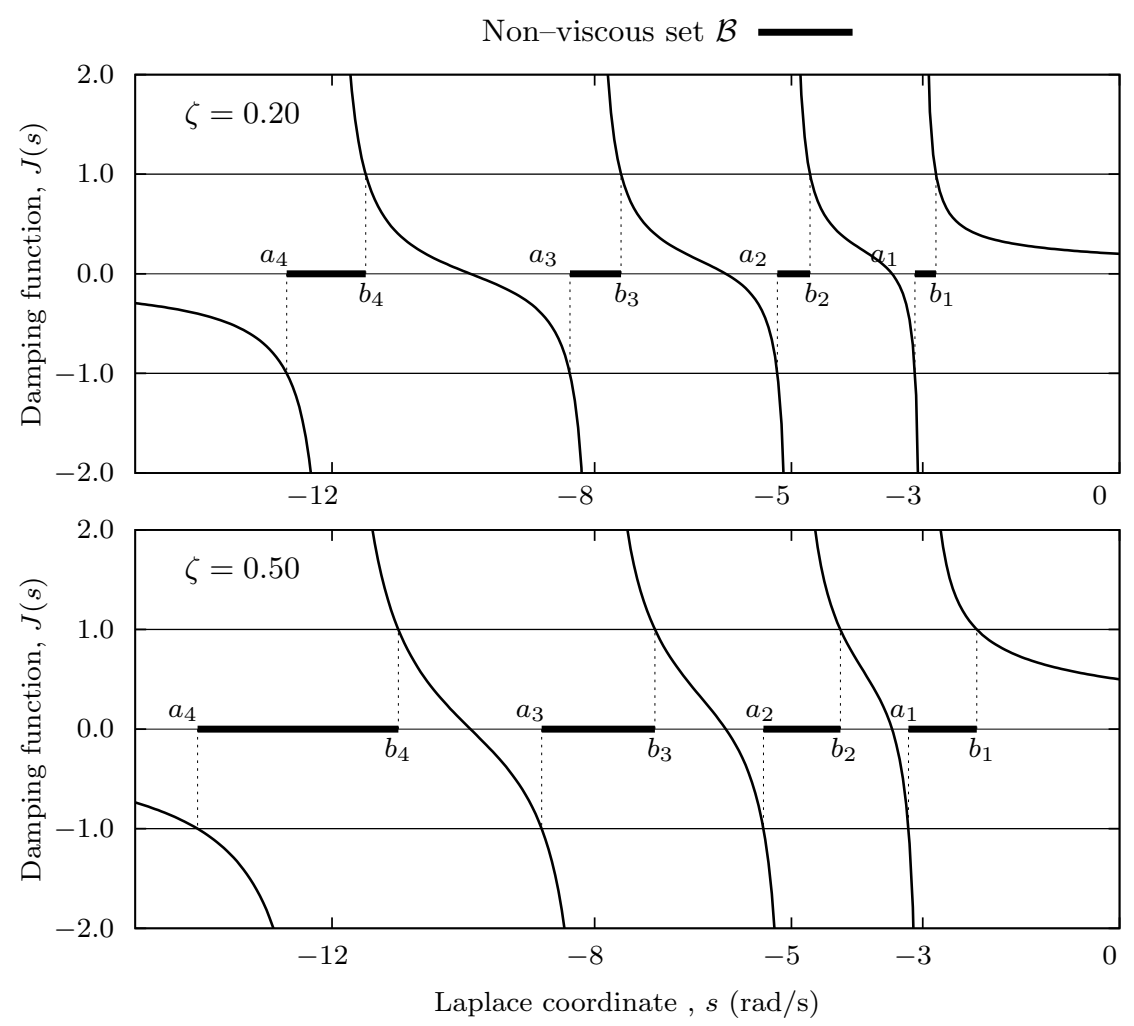

Figure 3.2: Dimensionless damping function $J(s)$ and intersection with ordinates 1 and -1 for $\mu_{j}=\{3,5,8,12\} \mathrm{rad} / \mathrm{s}$.

verified that

$$
\begin{aligned}
\lim _{\epsilon \rightarrow 0^{+}} J\left(-\mu_{j}+\epsilon\right) & =\lim _{\epsilon \rightarrow 0^{+}} \frac{\zeta}{N} \sum_{k=1}^{N} \frac{\mu_{k}}{-\mu_{j}+\mu_{k}+\epsilon} \\
& =\lim _{\epsilon \rightarrow 0^{+}} \frac{\zeta \mu_{j}}{N \epsilon}+\frac{\zeta}{N} \sum_{\substack{k=1 \\
k \neq j}}^{N} \frac{\mu_{k}}{\mu_{k}-\mu_{j}}=+\infty \\
\lim _{\epsilon \rightarrow 0^{+}} J\left(-\mu_{j}-\epsilon\right) & =\lim _{\epsilon \rightarrow 0^{+}} \frac{\zeta}{N} \sum_{k=1}^{N} \frac{\mu_{k}}{-\mu_{j}+\mu_{k}-\epsilon} \\
& =-\lim _{\epsilon \rightarrow 0^{+}} \frac{\zeta \mu_{j}}{N \epsilon}+\frac{\zeta}{N} \sum_{\substack{k=1 \\
k \neq j}}^{N} \frac{\mu_{k}}{\mu_{k}-\mu_{j}}=-\infty
\end{aligned}
$$


and therefore, the points close to $s=-\mu_{j}$ are always contained in $\mathcal{B}$. Naming $\mathcal{B}_{j}=\left[a_{j}, b_{j}\right]$ to the interval associated with $-\mu_{j}$, it is verified that

$$
\mathcal{B}=\bigcup_{j=1}^{N} \mathcal{B}_{j}=\bigcup_{j=1}^{N}\left[a_{j}, b_{j}\right]
$$

Since $J(s)$ is continuous, continuously differentiable and monotonically decreasing (except at $s=-\mu_{j}$ for $\left.1 \leq j \leq N\right)$, the right limits $b_{j}>-\mu_{j}$ are obtained as solution of $J(s)=+1$; the left limits $a_{j}<-\mu_{j}$ as solution of $J(s)=-1$. Furthermore, the intervals do not intersect each other, $\mathcal{B}_{j} \cap \mathcal{B}_{k}=\emptyset$ for $j \neq k$. The intervals' limits are directly related with the damping ratio so that $b_{j}-a_{j}$ decreases with $\zeta$, becoming zero, i.e. $a_{j}, b_{j} \rightarrow-\mu_{j}$, in the limit $\zeta \rightarrow 0$. This trend can be observed in Figs. 3.2 and will later be demonstrated.

The above presented developments can be generalized for multiple-dof structures with proportional damping matrix. The response is a $p$-dimensional vector $\mathbf{u}(t) \in$ $\mathbb{R}^{p}$ verifying the system of integro-differential equations

$$
\mathbf{M} \ddot{\mathbf{u}}+\int_{-\infty}^{t} \mathcal{G}(t-\tau) \dot{\mathbf{u}} \mathrm{d} \tau+\mathbf{K u}=\mathbf{F}(t)
$$

where $\mathbf{M}, \mathbf{K} \in \mathbb{R}^{p \times p}$ are the assembled mass and stiffness matrices. The time function $\mathcal{G}(t) \in \mathbb{R}^{p \times p}$ is the viscoelastic damping matrix and contains the hereditary functions controlling the dissipative forces. In proportional damping it is assumed that $\mathbf{M}, \mathcal{G}(t), \mathbf{K}$ become diagonal in the orthogonal base of the undamped problem, say $\left\{\phi_{1}, \ldots, \phi_{p}\right\}$; a detailed study on the conditions for proportional damping in non-viscous systems can be found in [29]. The response can be then decomposed in terms of the modal coordinates as $\mathbf{u}(t)=\sum_{i=1}^{p} \phi_{i} q_{i}(t)$. The well known orthogonal eigenrelations are $\boldsymbol{\phi}_{i}^{T} \mathbf{M} \boldsymbol{\phi}_{k}=m_{i} \delta_{i k}, \boldsymbol{\phi}_{i}^{T} \mathbf{K} \boldsymbol{\phi}_{k}=k_{i} \delta_{i k}$ and $\boldsymbol{\phi}_{i}^{T} \mathcal{G} \boldsymbol{\phi}_{k}=\mathcal{G}_{i}(t) \delta_{i k}$, where $\delta_{i k}$ is the Kronecker delta, $m_{i}, k_{i}$ and $\mathcal{G}_{i}(t)$ are the mass, stiffness and damping function associated to the mode $i$, respectively. These relations allow us to reduce the homogeneous Eq. (3.16) for $\mathbf{F}(t)=\mathbf{0}$ to $p$ single-dof free oscillators in $q_{i}(t)$ for $1 \leq i \leq p$

$$
m_{i} \ddot{q}_{i}+\int_{-\infty}^{t} \mathcal{G}_{i}(t-\tau) \dot{q}_{i}(\tau) \mathrm{d} \tau+k_{i} q_{i}=0
$$

Following the same procedure as before for single dof's, Eq. (3.17) is transformed into the nonlinear equation

$$
s^{2}+2 s J_{i}(s) \omega_{i}+\omega_{i}^{2}=0
$$

where $\omega_{i}=\sqrt{k_{i} / m_{i}}$ is the $i$ th natural frequency and $J_{i}(s)=G_{i}(s) / 2 m_{i} \omega_{i}$ is the $i$ th modal dimensionless damping function. It is clear that any real eignevalue 
$\sigma \in \mathbb{R}^{-}$of Eq. (3.18) verifies $\left|J_{i}(\sigma)\right| \geq 1$. Using a parallel notation, the number of non-viscous eigenvalues is $N_{i}$ and the non-viscous set associated to the mode $i$ is defined as $\mathcal{B}^{(i)}=\left\{s \in \mathbb{R}^{-}:\left|G_{i}(s)\right| \geq 2 m_{i} \omega_{i}\right\}=\cup_{j=1}^{N_{i}} \mathcal{B}_{j}^{(i)}$. Consequently the complete non-viscous set of a structure with a damping function defined by exponential kernels

$$
\mathcal{B}=\bigcup_{i=1}^{p} \mathcal{B}^{(i)}=\bigcup_{i=1}^{p} \bigcup_{j=1}^{N_{i}} \mathcal{B}_{j}^{(i)}=\bigcup_{i=1}^{p} \bigcup_{j=1}^{N_{i}}\left[a_{j}^{(i)}, b_{j}^{(i)}\right]
$$

Since the analysis of proportional damping systems can be reduced to a single dof oscillator, hereinafter the proposed method for the calculation of $a_{j}$ and $b_{j}$ and that for the computation of the non-viscous eigenvalues will be presented using the notation of the single dof system.

\subsubsection{Exact Limits of the Non-viscous Set}

It has been shown that the $j$ th interval limits $a_{j}, b_{j}$ around the pole $-\mu_{j}$ are functions of the damping parameters and can be calculated as the roots of the equations (negative for $a_{j}$ and positive for $b_{j}$ )

$$
\frac{\zeta}{N} \sum_{j=1}^{N} \frac{\mu_{j}}{s+\mu_{j}}=-1, \quad \frac{\zeta}{N} \sum_{j=1}^{N} \frac{\mu_{j}}{s+\mu_{j}}=+1
$$

Each of the equations can be reduced to an $N$ th-order polynomial simply multiplying both sides by $\prod_{j=1}^{N}\left(s+\mu_{j}\right)$. Exact solutions are available only for values $1 \leq N \leq 4$. In particular, polynomials $N=1,2$ allow the direct extraction and discussion of $a_{j}, b_{j}$ due to the simplicity of the resulting expressions; therefore, these polynomials will be analyzed separately. Although for $N=3,4$ analytical solutions can also be found, their roots (third and fourth order polynomials) are not manegable and do not provided useful additional information.

Case $N=1$ : One exponential kernel. The damping function includes a single kernel and consequently only one relaxation parameter $\mu$. The set $\mathcal{B}=[a, b]$ is a closed interval so that limits $a<-\mu<b$ for $\zeta>0$ are solutions of Eqs. (3.20) particularized with $N=1$

$$
\begin{aligned}
& \zeta \frac{\mu}{s+\mu}=-1 \quad \rightarrow \quad s=-\mu(1+\zeta) \equiv a \\
& \zeta \frac{\mu}{s+\mu}=+1 \quad \rightarrow \quad s=-\mu(1-\zeta) \equiv b
\end{aligned}
$$

While the left limit is always a negative number, the right one lies inside the positive real numbers set if $\zeta \geq 1$. By definition $\mathcal{B} \subset \mathbb{R}^{-}$, therefore the right limit 
can suitably be redefined as $b=\min \{0,-\mu(1-\zeta)\}$. Finally, the set $\mathcal{B}$ is defined as

$$
\mathcal{B}= \begin{cases}{[-(1+\zeta) \mu,-(1-\zeta) \mu]} & \text { if } 0<\zeta<1 \\ {[-(1+\zeta) \mu, 0]} & \text { if } \zeta \geq 1\end{cases}
$$

Notice that the interval tends to point $-\mu$ when $\zeta \rightarrow 0$; on the contrary the size of $\mathcal{B}$ increases with $\zeta$ so that $\lim _{\zeta \rightarrow \infty} \mathcal{B}=\mathbb{R}^{-}$

Case $N=2$ : Two exponential kernels. Without loss of generality, let us assume that the relaxation parameters are $0<\mu_{1}<\mu_{2}$. The non-viscous set is then formed by the union of two closed intervals $\mathcal{B}_{1}=\left[a_{1}, b_{1}\right]$ and $\mathcal{B}_{2}=\left[a_{2}, b_{2}\right]$ verifying $a_{j}<-\mu_{j}<b_{j}$ for $j=1,2$.

The left limits $a_{1}, a_{2}$ are the roots of

$$
\frac{\zeta}{2}\left(\frac{\mu_{1}}{s+\mu_{1}}+\frac{\mu_{2}}{s+\mu_{2}}\right)=-1
$$

Multiplying the previous equation by $\left(s+\mu_{1}\right)\left(s+\mu_{2}\right)$ after straight operations the second order polynomial is

$$
s^{2}+s(2+\zeta) \mu_{m}+(1+\zeta) \mu_{g}^{2}=0
$$

where $\mu_{m}=\left(\mu_{1}+\mu_{2}\right) / 2$ and $\mu_{g}=\sqrt{\mu_{1} \mu_{2}}$ are the arithmetic and geometric means of the relaxation parameters. The roots of Eq. (3.23) can be expressed as

$$
s_{1}, s_{2}=-\frac{\mu_{m}}{2}(2+\zeta)(1 \pm \sqrt{1-\eta})
$$

where

$$
\eta \equiv \frac{4(1+\zeta)}{(2+\zeta)^{2}}\left(\frac{\mu_{g}}{\mu_{m}}\right)^{2}
$$

is a bounded auxiliary value $0<\eta<1$. Indeed since the inequality $\mu_{g}<\mu_{m}$ always holds, $\eta$ verifies

$$
\eta=\frac{4+4 \zeta}{4+4 \zeta+\zeta^{2}}\left(\frac{\mu_{g}}{\mu_{m}}\right)^{2}<1 \cdot 1=1
$$

The roots from Eq. (3.24) are always negative for any value of the damping parameters. Since $-\mu_{1}>-\mu_{2}$, the left limit must verify $0>a_{1}>a_{2}$, and their expressions are

$$
a_{1}, a_{2}=-\frac{\mu_{m}}{2}(2+\zeta)(1 \mp \sqrt{1-\eta})
$$


The right limits $b_{2}<b_{1}<0$ are the roots of

$$
\frac{\zeta}{2}\left(\frac{\mu_{1}}{s+\mu_{1}}+\frac{\mu_{2}}{s+\mu_{2}}\right)=+1
$$

By the same procedure as before, the following second order polynomial is deduced

$$
s^{2}+s(2-\zeta) \mu_{m}+(1-\zeta) \mu_{g}^{2}=0
$$

with roots

$$
s_{1}, s_{2}=-\frac{1}{2}\left[(2-\zeta) \mu_{m} \pm \sqrt{(2-\zeta)^{2} \mu_{m}^{2}-4(1-\zeta) \mu_{g}^{2}}\right]
$$

In order to assign $s_{1}$ and $s_{2}$ to the limits $b_{1}, b_{2}$ the inequality $b_{2}<b_{1}<0$ and the different values of $\zeta$ must be into account. A more detailed analysis requires three different cases i) to iii) with the help of the function

$$
\rho=\frac{4|1-\zeta|}{(2-\zeta)^{2}}\left(\frac{\mu_{g}}{\mu_{m}}\right)^{2}
$$

i) Range $0<\zeta \leq 1$ for which $|1-\zeta|=1-\zeta$ and using the same reasoning as before, $0<\rho \leq 1$ can be easily proved. Indeed

$$
\rho=\frac{4-4 \zeta}{4-4 \zeta+\zeta^{2}}\left(\frac{\mu_{g}}{\mu_{m}}\right)^{2}<1 \cdot 1=1
$$

Therefore, since $0>b_{1}>b_{2}$ the right limits take the following values as function of $\rho$

$$
b_{1}, b_{2}=-\frac{\mu_{m}}{2}(2-\zeta)(1 \mp \sqrt{1-\rho})
$$

ii) Range $1<\zeta \leq 2$. When $\zeta=1$, Eq. (3.33) gives $b_{1}=0, b_{2}=-\mu_{m}$. When $\zeta>1$ one of the roots from Eq. (3.30) remains negative and the other positive. In the range of study, $|1-\zeta|=-(1-\zeta)$ and the roots are

$$
s_{1}, s_{2}=-\frac{\mu_{m}}{2}(2-\zeta)(1 \pm \sqrt{1+\rho})
$$

Since $\mathcal{B}$ must be always negative and again $b_{1}>b_{2}$, the positive root can not be assigned to any limit. If the negative root is assigned to $b_{2}$ then

$$
b_{1}=0, \quad b_{2}=-\frac{\mu_{m}}{2}(2-\zeta)(1+\sqrt{1+\rho})
$$


iii) Range $2<\zeta<+\infty$. Again $|1-\zeta|=-(1-\zeta)$ but $2-\zeta$ is now negative. The roots can be rewritten as

$$
s_{1}, s_{2}=-\frac{\mu_{m}}{2}(\zeta-2)(-1 \pm \sqrt{1+\rho})
$$

Rejecting the positive root again and assigning the negative one to $b_{2}$, the limits are

$$
b_{1}=0, \quad b_{2}=-\frac{\mu_{m}}{2}(\zeta-2)(\sqrt{1+\rho}-1)
$$

The results are arranged in the Table 3.1. Note in the first row that $\lim _{\zeta \rightarrow 0} \mathcal{B}=$

\begin{tabular}{lcc} 
& \multicolumn{2}{c}{$\mathcal{B}_{1}$} \\
\cline { 2 - 3 } & $a_{1}$ & $b_{1}$ \\
\hline$\zeta \rightarrow 0$ & $-\mu_{1}$ & $-\mu_{1}$ \\
$0<\zeta \leq 1$ & $-\frac{\mu_{m}}{2}(2+\zeta)(1-\sqrt{1-\eta})$ & $-\frac{\mu_{m}}{2}(2-\zeta)(1-\sqrt{1-\rho})$ \\
$1<\zeta \leq 2$ & $-\frac{\mu_{m}}{2}(2+\zeta)(1-\sqrt{1-\eta})$ & 0 \\
$2<\zeta<\infty$ & $-\frac{\mu_{m}}{2}(2+\zeta)(1-\sqrt{1-\eta})$ & 0 \\
$\zeta \rightarrow \infty$ & $-\frac{\mu_{g}^{2}}{\mu_{m}}$ & 0 \\
\hline & \multicolumn{2}{c}{$\mathcal{B}_{2}$} \\
& $a_{2}$ & $b_{2}$ \\
\hline$\zeta \rightarrow 0$ & $-\mu_{2}$ & $-\mu_{2}$ \\
$0<\zeta \leq 1$ & $-\frac{\mu_{m}}{2}(2+\zeta)(1+\sqrt{1-\eta})$ & $-\frac{\mu_{m}}{2}(2-\zeta)(1+\sqrt{1-\rho})$ \\
$1<\zeta \leq 2$ & $-\frac{\mu_{m}}{2}(2+\zeta)(1+\sqrt{1-\eta})$ & $-\frac{\mu_{m}}{2}(2-\zeta)(1+\sqrt{1+\rho})$ \\
$2<\zeta<\infty$ & $-\frac{\mu_{m}}{2}(2+\zeta)(1+\sqrt{1-\eta})$ & $-\frac{\mu_{m}}{2}(\zeta-2)(\sqrt{1+\rho}-1)$ \\
$\zeta \rightarrow \infty$ & $-\infty$ & $-\frac{\mu_{g}^{2}}{\mu_{m}}$ \\
\hline
\end{tabular}

Table 3.1: Exact solutions for limits of non-viscous set $\mathcal{B}=\mathcal{B}_{1} \cup \mathcal{B}_{2}$ with a model of two exponential kernels. Expressions of $\eta, \rho$ from Eqs. (3.25) and (3.31)

$\left\{-\mu_{1},-\mu_{2}\right\}$ and in this case the non-viscous set is reduced to two points. In the last row $\lim _{\zeta \rightarrow \infty} \mathcal{B}=\mathbb{R}^{-}$, result that can be extended to any number $N$ of exponential kernels.

\subsubsection{Approximated Limits of the Non-visocus Set}

Analytical solutions for the general case of $N>4$ exponential kernels are not available; in the present paper and exploiting the physical meaning of the damping ratio, approximated solutions are proposed. In general, $\zeta>0$, however in practice for the majority of real physical systems $0<\zeta \leq 1$. Therefore, if the limits of the $j$ th non-viscous subset $\mathcal{B}_{j}=\left[a_{j}, b_{j}\right]$ are considered as functions of the parameter 
$\zeta$, then $a_{j}(\zeta), b_{j}(\zeta)$ can be expanded around $\zeta=0$ as

$$
\begin{aligned}
& a_{j}(\zeta)=a_{j}(0)+\frac{\partial a_{j}(0)}{\partial \zeta} \zeta+\cdots+\frac{\partial^{n} a_{j}(0)}{\partial \zeta^{n}} \frac{\zeta^{n}}{n !}+\mathcal{O}\left(\zeta^{n+1}\right) \\
& b_{j}(\zeta)=b_{j}(0)+\frac{\partial b_{j}(0)}{\partial \zeta} \zeta+\cdots+\frac{\partial^{n} b_{j}(0)}{\partial \zeta^{n}} \frac{\zeta^{n}}{n !}+\mathcal{O}\left(\zeta^{n+1}\right)
\end{aligned}
$$

It is expected that the Taylor approximation will provide good results in the mentioned damping ratio range, but becoming poorer when $\zeta$ approximates the unity. The objective is now to obtain the expansion coefficients of Eqs. (3.38). For that, evaluating Eqs. (3.20) at $s=a_{j}(\zeta), b_{j}(\zeta)$ results in

$$
\frac{\zeta}{N} \sum_{k=1}^{N} \frac{\mu_{k}}{a_{j}(\zeta)+\mu_{k}}=-1, \quad \frac{\zeta}{N} \sum_{k=1}^{N} \frac{\mu_{k}}{b_{j}(\zeta)+\mu_{k}}=+1
$$

Multiplying these equations by $a_{j}(\zeta)+\mu_{j}$ and $b_{j}(\zeta)+\mu_{j}$, respectively, the following implicit equations in $a_{j}(\zeta)$ and $b_{j}(\zeta)$ can be obtained.

$$
\begin{aligned}
f(\zeta) & \equiv \mu_{j} \frac{\zeta}{N}+\left[\frac{\zeta}{N} H_{j}\left(a_{j}(\zeta)\right)+1\right]\left[a_{j}(\zeta)+\mu_{j}\right]=0 \\
g(\zeta) & \equiv \mu_{j} \frac{\zeta}{N}+\left[\frac{\zeta}{N} H_{j}\left(b_{j}(\zeta)\right)-1\right]\left[b_{j}(\zeta)+\mu_{j}\right]=0
\end{aligned}
$$

where the function $H_{j}(s)$ is defined as

$$
H_{j}(s)=\sum_{\substack{k=1 \\ k \neq j}}^{N} \frac{\mu_{k}}{s+\mu_{k}}
$$

Since $f(\zeta), g(\zeta)$ are identically null for any value of $\zeta$, their derivatives up to any order also vanish

$$
f^{\prime}(\zeta)=0, \quad f^{\prime \prime}(\zeta)=0, \ldots \quad g^{\prime}(\zeta)=0, \quad g^{\prime \prime}(\zeta)=0, \ldots
$$

From $f(0)=g(0)=0$, the first terms of the expansions $a_{j}(0)=b_{j}(0)=-\mu_{j}$ can directly be obtained. The successive derivatives of $a_{j}(\zeta), b_{j}(\zeta)$ evaluated at $\zeta=0$ can recursively be computed from $f^{\prime}(0)=f^{\prime \prime}(0)=f^{\prime \prime \prime}(0)=0, \ldots$. After some operations the first four derivatives are

$$
\begin{aligned}
\frac{\partial a_{j}(0)}{\partial \zeta}=-\frac{\partial b_{j}(0)}{\partial \zeta} & =-\frac{\mu_{j}}{N} \\
\frac{\partial^{2} a_{j}(0)}{\partial \zeta^{2}}=\frac{\partial^{2} b_{j}(0)}{\partial \zeta^{2}} & =\frac{2 \mu_{j}}{N^{2}} H_{j}\left(-\mu_{j}\right) \\
\frac{\partial^{3} a_{j}(0)}{\partial \zeta^{3}}=-\frac{\partial^{3} b_{j}(0)}{\partial \zeta^{3}} & =-\frac{6 \mu_{j}}{N^{3}}\left[H_{j}^{2}\left(-\mu_{j}\right)+\mu_{j} \frac{\partial H_{j}\left(-\mu_{j}\right)}{\partial s}\right] \\
\frac{\partial^{4} a_{j}(0)}{\partial \zeta^{4}}=\frac{\partial^{4} b_{j}(0)}{\partial \zeta^{4}} & =\frac{12 \mu_{j}}{N^{4}}\left[2 H_{j}^{3}\left(-\mu_{j}\right)\right. \\
& \left.+6 \mu_{j} H_{j}\left(-\mu_{j}\right) \frac{\partial H_{j}\left(-\mu_{j}\right)}{\partial s}+\mu_{j}^{2} \frac{\partial^{2} H_{j}\left(-\mu_{j}\right)}{\partial s^{2}}\right]
\end{aligned}
$$


By recursion, the $n$th derivatives $\partial^{n} a_{j}(0) / \partial \zeta^{n}$ and $\partial^{n} b_{j}(0) / \partial \zeta^{n}$ can be calculated from $f^{(n)}(0)=g^{(n)}(0)=0$ using the information obtained in the previous step. As shown in Eq. (3.43) the results will depend on the powers and derivatives of $H_{j}(s)$ evaluated at $s=-\mu_{j}$

$$
\frac{\partial^{n} H_{j}\left(-\mu_{j}\right)}{\partial s^{n}}=\sum_{\substack{k=1 \\ k \neq j}}^{N} \frac{(-1)^{n} n ! \mu_{k}}{\left(\mu_{k}-\mu_{j}\right)^{n+1}}
$$

\subsection{Numerical Computation of Non-viscous Eigenvalues}

Since the damping function with $N$ exponential kernels is given by Eq. (3.13), the non-viscous eigenvalues are the $N$ real roots of Eq. (3.5). The terms $s+\mu_{j}$ for $1 \leq j \leq N$ are at the damping function denominators; possible singularities are avoided multiplying Eq. (3.5) by $\prod_{j=1}^{N}\left(s+\mu_{j}\right)$, obtaining the $N+2$ th order polynomial

$$
P(s)=\left(s^{2}+\omega_{n}^{2}\right) \prod_{j=1}^{N}\left(s+\mu_{j}\right)+2 s \omega_{n} \frac{\zeta}{N} \sum_{j=1}^{N} \mu_{j} \prod_{\substack{k=1 \\ k \neq j}}^{N}\left(s+\mu_{k}\right)
$$

There are many iterative methods available to obtain the $N$ real roots of the previous polynomial. However, in the present paper we are interested in the onestep methods to estimate the solution using an explicit expression.

\subsubsection{Existing One-Step Methods}

To the best of our knowledge, two one-step estimations exist: one due to Adhikari and Pascual [31] and another recently proposed by Lázaro and Pérez-Aparicio [63]. The former applies the first iteration of Newton's method with $s=-\mu_{j}$ as the initial point. Assuming that $-\mu_{j}+\Delta_{j}$ is close to the solution, $\Delta_{j}$ can be explicitly calculated by expanding $P(s)$ up to the first oder around the initial point

$$
0 \approx P\left(-\mu_{j}+\Delta_{j}\right) \approx P\left(-\mu_{j}\right)+\frac{\partial P\left(-\mu_{j}\right)}{\partial s} \Delta_{j}
$$

After some simplifications, the expressions of [31] can be rewritten in terms of the current notation as

$$
\sigma_{j} \approx-\mu_{j}-\frac{P\left(-\mu_{j}\right)}{\frac{\partial P\left(-\mu_{j}\right)}{\partial s}}=-\mu_{j}+\frac{\mu_{j}^{2} p_{j}}{\mu_{j} p_{j}-\mu_{j}\left(r_{j}+\mu_{j} q_{j}\right)+\frac{N p_{j}}{2 \omega_{n} \zeta}\left(\mu_{j}^{2}+\omega_{n}^{2}\right)}
$$


where

$$
p_{j}=\prod_{\substack{k=1 \\ k \neq j}}^{N}\left(\mu_{k}-\mu_{j}\right), \quad q_{j}=\sum_{\substack{k=1 \\ k \neq j}}^{N} \prod_{\substack{r=1 \\ r \neq j, k}}^{N}\left(\mu_{r}-\mu_{j}\right), \quad r_{j}=\sum_{\substack{k=1 \\ k \neq j}}^{N} \mu_{j} \prod_{\substack{r=1 \\ r \neq j, k}}^{N}\left(\mu_{r}-\mu_{j}\right)
$$

Assuming light damping $\zeta \ll 1$ the root always lies close to $-\mu_{j}$, therefore it is expected that the solution from Eq. (3.48) represents a close approximation.

The reference [63] considers the eigenvalues as functions of the damping parameters. In particular, assuming that the $j$ th non-viscous eigenvalue $\sigma_{j}=\sigma_{j}(\zeta)$ can be written in terms of the damping ratio, a new approximation based on a Taylor expansion up to the second order is proposed

$$
\begin{aligned}
\sigma_{j} & \approx \sigma_{j}(0)+\frac{\partial \sigma_{j}(0)}{\partial \zeta} \zeta+\frac{\partial^{2} \sigma_{j}(0)}{\partial \zeta^{2}} \frac{\zeta^{2}}{2} \\
& =-\mu_{j}+\frac{2 \mu_{j}^{2} \omega_{n} \zeta}{N\left(\mu_{j}^{2}+\omega_{n}^{2}\right)}+\frac{4 \mu_{j}^{3} \omega_{n}^{2} \zeta^{2}}{N^{2}\left(\mu_{j}^{2}+\omega_{n}^{2}\right)^{2}}\left[\frac{\mu_{j}^{2}-\omega_{n}^{2}}{\mu_{j}^{2}+\omega_{n}^{2}}+H_{j}\left(-\mu_{j}\right)\right]
\end{aligned}
$$

where $H_{j}(s)$ is the function defined by Eq. (3.41). As in [31], this expression accurately estimates the exact eigenvalue around $\zeta=0$, i.e. for lightly damped systems. Moreover, the error between the approximation Eq. (3.49) and that of the exact one is bounded by a term $\mathcal{O}\left(\zeta^{3}\right)$.

\subsubsection{Proposed Method}

In this subsection, the question Q3 pointed in Sec. 3.2 is addressed. As proved in Section 3.2 , each non-viscous eigenvalue is bounded by an interval $\left[a_{j}, b_{j}\right]$, where the limits $a_{j}, b_{j}$ can be approximated by Eqs. (3.38), (3.43) for the general case of $N$ exponential kernels. The main objective is to build a new one-step numerical approximation of $\sigma_{j}$ using the information provided by its non-viscous set $\mathcal{B}_{j}=$ $\left[a_{j}, b_{j}\right]$. In order to avoid singularities, we define a new characteristic equation associated with the $j$ th non-viscous multiplying Eq. (3.5) by $s+\mu_{j}$, resulting

$$
D_{j}(s)=\left(s^{2}+\omega_{n}^{2}\right)\left(s+\mu_{j}\right)+2 s \omega_{n} \frac{\zeta}{N}\left[\mu_{j}+\left(s+\mu_{j}\right) H_{j}(s)\right]=0
$$

The function $D_{j}(s)$ vanishes at $s=\sigma_{j}$ and is continuous with continuous first derivative at $s=-\mu_{j}$ but not at $s=-\mu_{k} \in \mathcal{B}_{k}, \forall 1 \leq k \leq N$ and for $k \neq j$. Furthermore, since $\mathcal{B}_{j} \cap \mathcal{B}_{k}=\emptyset, D_{j}(s)$ is also continuously differentiable inside $\mathcal{B}_{j}$. The key idea is to quadratically interpolate $D_{j}(s)$ with three data points $s=a_{j},-\mu_{j}, b_{j}$. Since exact expressions for $a_{j}, b_{j}$ are not available for $N$ kernels, it is necessary to use the approximated expressions obtained above. Taking the linear 
expression $a_{j}, b_{j} \approx-\mu(1 \pm \zeta / N)$ the approximated and sought $j$ th characteristic equation can be written in terms of Lagrange's polynomials as

$$
D_{j}(s) \approx D_{j a} L_{a}(s)+D_{j \mu} L_{\mu}(s)+D_{j b} L_{b}(s) \equiv \tilde{D}_{j}(s)
$$

where the polynomials are

$$
\begin{aligned}
L_{a}(s), L_{b}(s) & =\frac{1}{2 \mu_{j}^{2} \nu^{2}}\left(s+\mu_{j}\right)\left[s+\mu_{j}(1 \mp \nu)\right] \\
L_{\mu}(s) & =\frac{1}{\mu_{j}^{2} \nu^{2}}\left[s+\mu_{j}(1-\nu)\right]\left[s+\mu_{j}(1+\nu)\right]
\end{aligned}
$$

The new variable $\nu=\zeta / N$ has been introduced for notation convenience. The values $D_{j a}, D_{j b}, D_{j \mu}$ are obtained evaluating $D_{j}(s)$ at the data points $s=-\mu_{j}(1 \pm$ $\nu),-\mu_{j}$

$$
\begin{aligned}
D_{j a} & =D_{j}\left[-\mu_{j}(1+\nu)\right]=-\mu_{j} \nu\left\{\left[\omega_{n}+\mu_{j}(1+\nu)\right]^{2}-2 \mu_{j} \omega_{n} \nu(1+\nu) H_{j a}\right\} \\
D_{j b} & =D_{j}\left[-\mu_{j}(1-\nu)\right]=\mu_{j} \nu\left\{\left[\omega_{n}-\mu_{j}(1-\nu)\right]^{2}-2 \mu_{j} \omega_{n} \nu(1-\nu) H_{j b}\right\} \\
D_{j \mu} & =D_{j}\left(-\mu_{j}\right)=-2 \mu_{j}^{2} \nu \omega_{n}
\end{aligned}
$$

and

$$
\begin{aligned}
& H_{j a}=H_{j}\left(-\mu_{j}(1+\nu)\right)=\sum_{\substack{k=1 \\
k \neq j}}^{N} \frac{\mu_{k}}{\mu_{k}-(1+\nu) \mu_{j}} \\
& H_{j b}=H_{j}\left(-\mu_{j}(1-\nu)\right)=\sum_{\substack{k=1 \\
k \neq j}}^{N} \frac{\mu_{k}}{\mu_{k}-(1-\nu) \mu_{j}}
\end{aligned}
$$

After some operations, the coefficients of the second order polynomial $\tilde{D}_{j}(s)$ can be expressed in the form of

$$
\tilde{D}_{j}(s)=C_{j}\left(s^{2}+2 \mu_{j} \alpha_{j} s+\mu_{j}^{2} \beta_{j}\right)
$$

where

$$
\begin{aligned}
C_{j} & =\frac{D_{j a}+D_{j b}-2 D_{j \mu}}{2 \mu_{j}^{2} \nu^{2}} \\
\alpha_{j} & =1+\frac{\nu}{2} \frac{D_{j b}-D_{j a}}{D_{j b}+D_{j a}-2 D_{j \mu}} \\
\beta_{j} & =1+\nu \frac{D_{j b}-D_{j a}+2 \nu D_{j \mu}}{D_{j b}+D_{j a}-2 D_{j \mu}}
\end{aligned}
$$


Assuming $D_{j a}+D_{j b}-2 D_{j \mu} \neq 0$, the non-viscous eigenvalue can be approximated by one of both roots of $\tilde{D}_{j}(s)=0$, thus

$$
\begin{aligned}
\sigma_{j} & \approx-\mu_{j}\left(\alpha_{j} \pm \sqrt{\alpha_{j}^{2}-\beta_{j}}\right) \\
& =-\mu_{j}\left(1+\frac{\nu}{2} \frac{D_{j b}-D_{j a} \pm \sqrt{\left(D_{j b}-D_{j a}-4 D_{j \mu}\right)^{2}-8 D_{j a} D_{j \mu}}}{D_{j b}+D_{j a}-2 D_{j \mu}}\right)
\end{aligned}
$$

The previous expressions give two real numbers provided that $\left(D_{j b}-D_{j a}-4 D_{j \mu}\right)^{2} \geq$ $8 D_{j a} D_{j \mu}$ but a priori it is not possible to know which one is the proposed estimation. Theoretically, the one closest to the exact non-viscous eigenvalue should be chosen, but this comparison is obviously not possible. In practice, the closest to the quadratic approximation from Eq. (3.49) is generally a good choice. Other selection criteria may be proposed, for example to adopt the root with lowest value $D_{j}(s)$ or the one lying inside the set $\mathcal{B}$, i.e. $\left|J\left(\sigma_{j}\right)\right| \geq 1$.

If $D_{j a}+D_{j b}-2 D_{j \mu}=0$, the interpolation curve degenerates into a straight line whose intersection with the horizontal axis is the approximation, and is given by

$$
\sigma_{j} \approx-\mu_{j}+\frac{4 \mu_{j}^{3} \nu^{2} \omega_{n}}{D_{j b}-D_{j a}}
$$

\subsection{Numerical Example}

In order to validate the theoretical developments, a single dof viscoelastic oscillator (see Fig. 3.1) with a four-exponential model is analyzed. The mass and linear stiffness of the system are $m=1 \mathrm{~kg}, k=100 \mathrm{~N} / \mathrm{m}$ and the non-viscous parameters $\mu_{j}=\{4,9,16,22\} \mathrm{rad} / \mathrm{s}$. Since the quality of the proposed methods is directly related with the damping level, the results are shown as function of this damping. The two contributions of this paper are presented separately: First, in Figs. 3.3 the limits of the non-viscous set are obtained from the proposed method and compared with the exact ones computed from polynomial root finding. Second, in Figs. 3.4, the approximated non-viscous eigenvalues are compared with the exact ones and with those from the one-step methods by [77] and [63].

As mentioned the model includes four exponential kernels, therefore for each $\zeta$ the non-viscous set is formed by the union of four closed intervals, $\mathcal{B}(\zeta)=$ $\cup_{j=1}^{4} \mathcal{B}_{j}(\zeta)=\cup_{j=1}^{4}\left[a_{j}(\zeta), b_{j}(\zeta)\right]$. The exact and approximated limits $a_{j}(\zeta), b_{j}(\zeta)$ from Eqs. (3.38), (3.43) are represented in Figs. 3.3 for $0 \leq \zeta \leq 1$, shading the 
region comprised by both limits to highlight the non-viscous subsets. Since for the great majority of practical materials the damping ratio lies inside the mentioned interval, the abscissa has been log-scaled for a clear representation. Let us denote by $l_{j}(\zeta)$ the length of $\mathcal{B}_{j}$, that is, the vertical distance between curves $a_{j}(\zeta), b_{j}(\zeta)$ in Figs. 3.3. Notice that $l_{j}(\zeta)$ increases with the damping level, see Figs. 3.2 left and right, therefore $\mathcal{B}_{j}$ becomes larger with damping. This fact can be mathematically verified if $l_{j}(\zeta)$ is computed from the quadratic approximation of the limits, see Eq. (3.43), obtaining

$$
\begin{aligned}
l_{j}(\zeta) & =b_{j}(\zeta)-a_{j}(\zeta) \\
& \approx\left[-\mu_{j}+\frac{\zeta}{N} \mu_{j}+\frac{\zeta^{2}}{N^{2}} H_{j}\left(-\mu_{j}\right)\right]-\left[-\mu_{j}-\frac{\zeta}{N} \mu_{j}+\frac{\zeta^{2}}{N^{2}} H_{j}\left(-\mu_{j}\right)\right] \\
& =\frac{2 \zeta}{N} \mu_{j}
\end{aligned}
$$

It can be observed that for this approximation order, $l_{j}(\zeta)$ is directly proportional to $\zeta$. Obviously an improved expression for the limits may be obtained adding higher order terms, although no relevant additional information would be obtained from a qualitative point of view. Since $l_{j}(\zeta)$ is also proportional to $\mu_{j}$, with $\zeta$ fixed the higher $\mu_{j}$ the wider the associated interval $\mathcal{B}_{j}$. This fact can also be appreciated in Figs. 3.2, where the intervals clearly become wider when moving away from the origin. On the contrary, when $\zeta \rightarrow 0$ the $\mathcal{B}_{j}$ intervals reduce to the four points $-\mu_{j}=\{-4,-9,-16,-22\}$ as predicted by the theory.

In Figs. 3.3, it can be appreciated that the linear approximation generates nonviscous intervals symmetric respect to $-\mu_{j}$ and satisfactorily predicts the exact limits through $0 \leq \zeta \leq 0.1$. If a similar accuracy is needed for a wider range of the damping level, higher orders terms must be added. Hence, the fourth order Taylor polynomial approximation has been plotted, observing that the validity range approximately extends to $0 \leq \zeta \leq 0.6$. After that, the approximation diverges and is not valid.

It is interesting to comment the influence of the non-viscous parameters in the approximation of the $j$ th non-viscous subset. The linear approximation is accurate close to $\zeta=0$, i.e. $a_{j}(\zeta), b_{j}(\zeta) \approx-\mu_{j}(1 \pm \zeta / N)$. These limits only depend on $\mu_{j}$ and not on the rest of $\mu_{k}, 1 \leq k \leq N$ and $j \neq k$, that appear in the higher order terms via $H_{j}(s)$ and its derivatives, Eq. (3.43). This dependency implies that the interaction between non-viscous parameters is relevant for high damping. Furthermore, several numerical cases examined have shown that if the non-viscous parameters are relatively close to each other, the quality of the proposed solutions is lower. This is due to the possibility of some of the $H_{j}(s)$ summands in the denominator becoming large, see Eqs. (3.41), (3.44). Therefore, high-order terms of $a_{j}(\zeta), b_{j}(\zeta)$ take a greater relevance and they can not be neglected. Otherwise, 

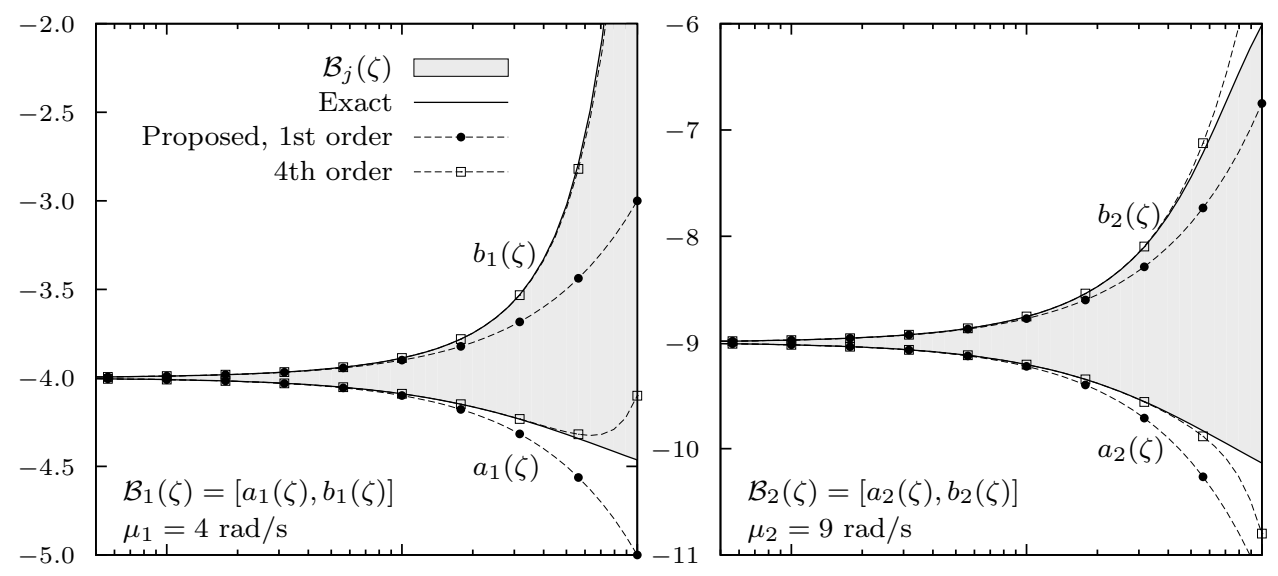

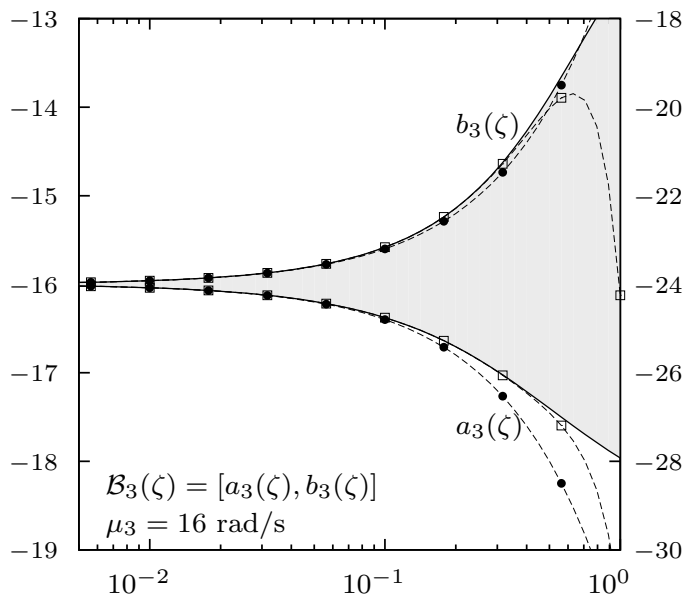

Damping ratio,$\zeta$

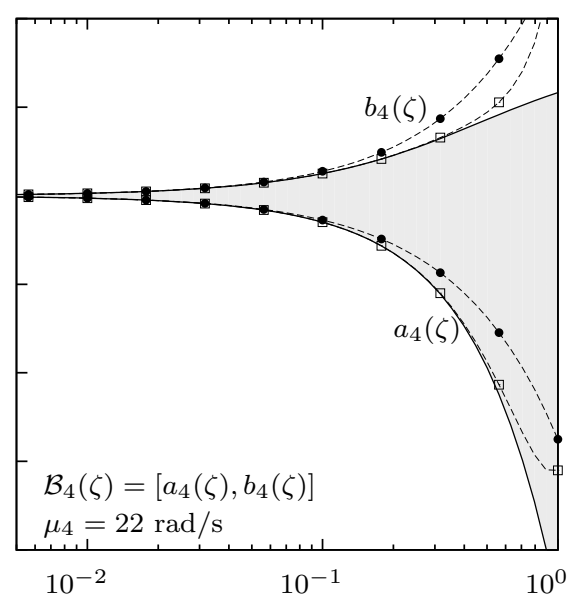

Damping ratio,$\zeta$

Figure 3.3: Non-viscous subsets $\mathcal{B}_{j}(\zeta)$ for $\mu_{j}=\{4,9,16,22\} \mathrm{rad} / \mathrm{s}$ as function of damping ratio $\zeta$. Exact and proposed limits $a_{j}(\zeta), b_{j}(\zeta)$ (1st and 4 th order Taylor approximations). 
when the set $\left\{\mu_{j}\right\}$ is uniformly distributed, the weight of terms associated with $H_{j}(s)$ is less important and the solution can be estimated with low-order terms.

As described before, the introduction of the new non-viscous set $\mathcal{B}$ has motivated us to search a novel one-step numerical procedure, to develop an explicit expression to estimate the non-viscous eigenvalues of the system. The method is based on the quadratic approximation of the characteristic equation inside the subset $\mathcal{B}_{j}$. In plots Fig. 3.4(a) to 3.4(d), the approximated non-viscous eigenvalues from the two published and the present methods have been represented together with the exact values. The latter methods are calculated by iteration of the characteristic Eq. (3.45). In Figs. 3.4(e) to 3.4(h), the relative percentage errors of the four non-viscous eigenvalues are shown using logarithmic scale. Since the accuracy of the three approaches is conditioned by the level of damping, the error increases with the damping ratio as observed in the figures. The relative minima are due to the coincidence of the approximated and exact curves at certain points. As predicted by the theory, the three methods present good fit with the exact values for low damping, $0 \leq \zeta \leq 0.1$ : The differences are imperceptible in this range, but the relative error of the proposed method is several orders of magnitude lower than the other two. Furthermore, the current method approximates well along all the range $0 \leq \zeta \leq 1$ with a generally lower relative error. Only for the fourth non-viscous eigenvalue and high damping, Figs. 3.4(d) and 3.4(h), better results are found with the solution of [63].

As shown for $a_{j}, b_{j}$, the eigenvalue numerical solutions depend on the set of nonviscous parameters, $\mu_{j}$ with $1 \leq j \leq N$. If their distribution is not uniform and the distances between them are relatively small, the function $H_{j}(s)$ values are relevant and contradict the hypothesis of regularity needed to substitute the characteristic Eq. (3.50) by a quadratic curve. Therefore, it is expected that for relatively small distances the results of any of the three approximated methods are questionable, specially for high damping ratios. This fact has been validated with several numerical examples not shown here.

\subsection{Conclusions}

One of the main differences between the analyses of viscous and non-viscous single degree-of-freedom dynamic oscillators is that the latter one can present negative real eigenvalues (named non-viscous) besides the two complex-conjugated roots. In this paper a new mathematical characterization of the non-viscous eigenvalues is proposed, introducing the novel concept of "non-viscous set". It can be assured that his set includes all non-viscous eigenvalues and it is formed by the union of 

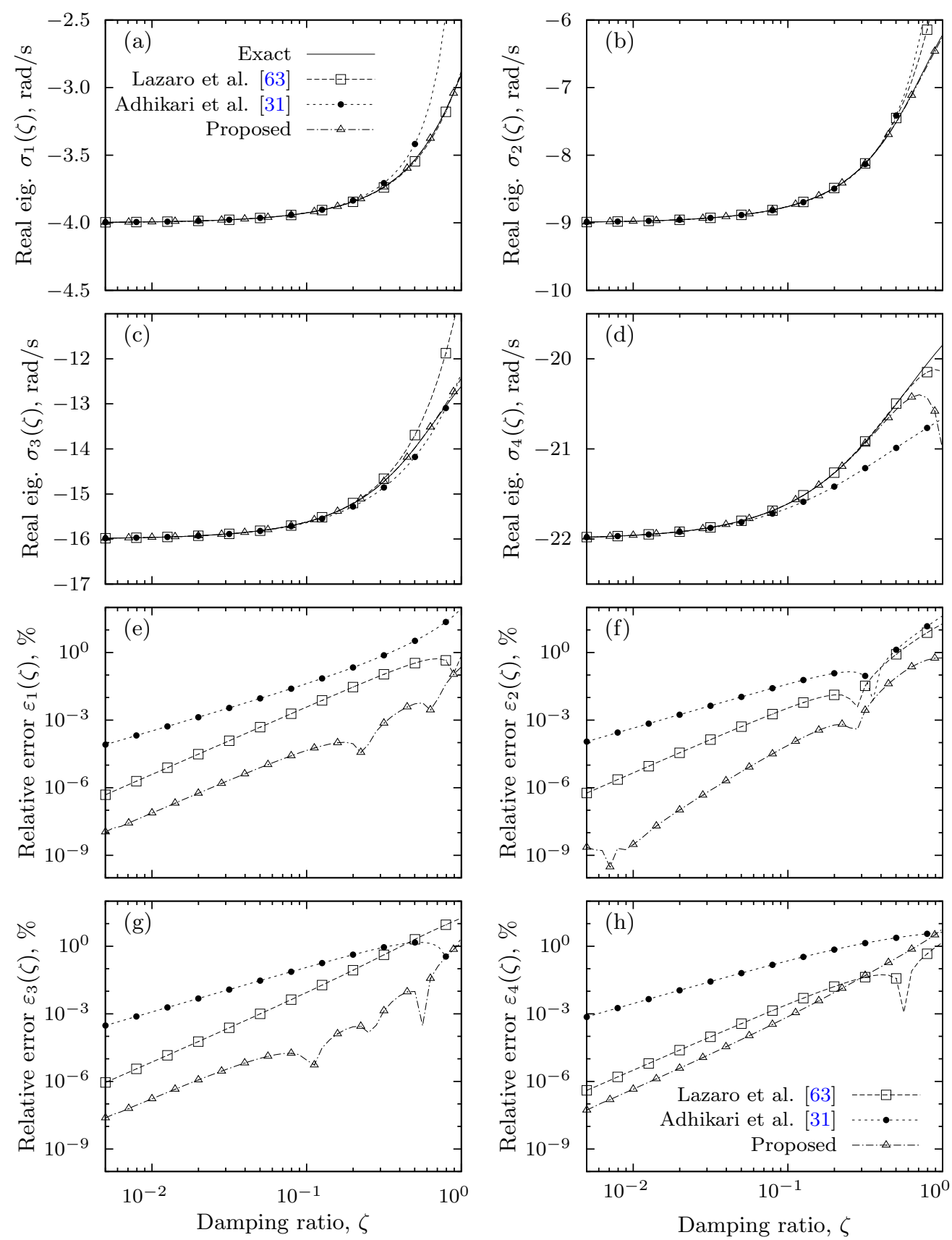

Figure 3.4: Approximated and exact non-viscous eigenvalues as function of damping ratio, figures (a)-(d). Relative error in percentage of approximated methods, figures (e)-(h). Reference [31] from Adhikari \& Pascual and [63] from Lázaro \& Pérez-Aparicio. 
closed intervals that are negative real numbers.

The developed theoretical results have been applied to the widespread Biot's model of damping, based on the assumption that the kernel hereditary function is a sum of $N$ exponentials. For this type of damping function, analytical expressions of the non-viscous set have been obtained for $N=1$ and 2 exponential kernels. For the general case $N>2$, approximated solutions based on the Taylor series expansion of the interval limits have been proposed, and in particular analytical solutions up to the fourth order are given.

The non-viscous set provides new information on the location of the non-viscous eigenvalues, allowing us to develop analytical expression for their estimation. Also, the new methodology for the computation of the $j$ th non-viscous eigenvalue is based on the approximation of the associated characteristic equation by a quadratic curve using Lagrange polynomials. Thus, the numerical approximation can be easily calculated as a second order polynomial root.

In order to validate the theoretical results, a numerical example under a four exponential kernels' model is analyzed. Firstly, the limits of the non-viscous subsets are calculated as function of damping ratio. It is also verified that the proposed fourth order solution estimates with great accuracy the exact limits in a wide range of the damping ratio. Secondly, exact and proposed non-viscous eigenvalues are obtained and compared with two published one-step numerical methods. It is shown that the proposed method not only is very close to the exact one in almost the complete range of damping, but also presents the lowest relative error for most of the studied cases with variable damping parameters. As predicted by the theory, the error increases with the oscillator damping level. New research oriented to find different properties of the real eigenvalues for multiple degree-of-freedom viscoelastic structures is currently being carried out, particularly for overdamped systems. 



\section{Bibliography}

[7] M.A. Biot. "Variational Principles in Irreversible Thermodynamics with Application to Viscoelasticity." In: Physical Review 97.6 (1955), 1463-1469 (cit. on pp. 3, 20, 24, 37, 58, 82, 93, 192).

[14] D.F. Golla and P.C. Hughes. "Dynamics of Viscoelastic Structures - A Time-domain, Finite-element Formulation." In: Journal of Applied MechanicsTransactions of the ASME 52.4 (1985), 897-906 (cit. on pp. 3, 20, 22, 24, $58,60,82,84,118,160,162,164,192)$.

[28] S. Adhikari. "Dynamics of Non-viscously Damped Linear Systems." In: Journal of Engineering Mechanics 128.3 (2002), 328-339 (cit. on pp. 4-6, $22,35,36,58,84,94,121,125,129,131,160-162,165,168,170,171,203)$.

[29] S. Adhikari. "Classical normal modes in non-viscously damped linear systems." In: AIAA Journal 39.5 (2001), pp. 978-980 (cit. on pp. 5, 35, 44, $63,84,121)$.

[30] J. Woodhouse. "Linear Damping Models For Structural Vibration." In: Journal of Sound and Vibration 215.3 (1998), 547-569 (cit. on pp. 5, 35, $58,121,170,171)$.

[31] Sondipon Adhikari and Blanca Pascual. "Eigenvalues of linear viscoelastic systems." In: Journal of Sound and Vibration 325.4-5 (2009), 1000-1011 (cit. on pp. 5, 21, 35, 41, 42, 69, 70, 76, 83, 119, 120, 141, 151, 152, 193).

[32] Sondipon Adhikari and Blanca Pascual. "Iterative Methods for Eigenvalues of Viscoelastic Systems." In: Journal of Vibration and Acoustics 133.2 (2011), pp. 021002.1-021002.7 (cit. on pp. 5, 21, 35, 42, 59, 83, 108, 119, $171,193)$.

[33] M. Lázaro, J. L. Pérez-Aparicio, and M. Epstein. "Computation of eigenvalues in proportionally damped viscoelastic structures based on the fixedpoint iteration." In: Applied Mathematics and Computation 219.8 (2012), 3511-3529 (cit. on pp. 7, 59, 119, 193).

[40] D.J. McTavish and P.C. Hughes. "Modeling of Linear Viscoelastic Space Structures." In: Journal of Vibration and Acoustics-Transactions of the ASME 115.1 (1993), 103-110 (cit. on pp. 20, 24, 58, 82, 118, 160, 164). 
[41] GA Lesieutre and DL Mingori. "Finite-Element Modeling of Frequencydependent Material Damping Using Augmenting Thermodynamic Fields." In: Journal of Guidance Control and Dynamics 13.6 (1990), 1040-1050 (cit. on pp. $20,24,58,82,119)$.

[54] A Muravyov and SG Hutton. "Closed-form solutions and the eigenvalue problem for vibration of discrete viscoelastic systems." In: Journal of Applied Mechanics-Transactions of the ASME 64.3 (1997), 684-691 (cit. on pp. 21, 58, 83, 119, 160, 164, 193).

[63] M. Lázaro and J. L. Pérez-Aparicio. "Parametric solutions of the eigenvalue problem for single degree-of-freedom viscoelastic systems." In: AIAA Journal. 0 (2013), pp. i-xvii (cit. on pp. 21, 59, 69, 70, 72, 75, 76, 193, 201).

[64] P Muller. "Are the eigensolutions of a l-d.o.f. system with viscoelastic damping oscillatory or not?" In: Journal of Sound and Vibration 285.12 (2005), 501-509 (cit. on pp. 22, 38, 58, 84, 121, 162).

[73] A Muravyov and SG Hutton. "Free vibration response characteristics of a simple elasto-hereditary system." In: Journal of Vibration and AcousticsTransactions of the ASME 120.2 (1998), 628-632 (cit. on pp. 38, 58, 160, 162).

[74] S Adhikari. "Qualitative dynamic characteristics of a non-viscously damped oscillator." In: Proceedings of the Royal Society A-Mathematical Physical and Engineering Sciences 461.2059 (2005), 2269-2288 (cit. on pp. 38, 58, 84, 121).

[76] S. Adhikari. "Dynamic response characteristics of a nonviscously damped oscillator." In: Journal of Applied Mechanics-Transactions of the ASME 75.1 (2008), pp. 011003.01-011003.12 (cit. on p. 58).

[77] S. Adhikari. "Experimental Identification Of Generalized Proportional Viscous Damping Matrix." In: Journal of Vibration and Acoustics 131.1 (2009), 11008.01-11008.12 (cit. on pp. 59, 72). 


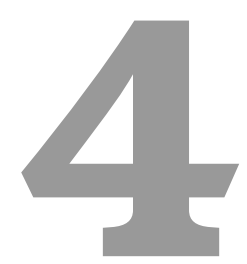

\section{Parametric solutions of the eigenvalue problem for single-degree-of-freedom viscoelastic systems}

\subsection{Introduction}

The importance of mathematical modeling for structural dynamic systems in fields such as Aerospace or Civil Engineering is well known. One of the most studied issues in the last 50 years has been the search of robust and efficient models for energy dissipation mechanisms. In this sense, the linear viscoelastic models have been profusely used, covering a wide range of structural systems. A large number of analysis methods for these models depend on the solution of the associated eigenvalue problem. This dependency has motivated us to investigate efficient numerical methods to solve the eigenvalue problem of linear viscoelastic systems.

The most general case of a viscoelastic system with one degree of freedom, say $u(t)$, can be modeled with a single mass, say $m$, attached to a fixed point by a viscoelastic constraint. Fig. 4.1 shows the schematic configuration mass-spring- 
viscoelastic damper and the corresponding free body diagram with the applied forces of the mass. Hence, the reaction force produced in the mass, say $R(t)$, is related with the displacement by

$$
R(t)=\int_{-\infty}^{t} \mathcal{G}(t-\tau) \dot{u}(\tau) \mathrm{d} \tau+k u(t)
$$

where $\mathcal{G}(t)$ is the dissipative kernel or damping function, characteristic of the considered viscoelastic model, and $k$ is the constant of the linear-elastic spring. Notice that the dissipative force depends on the past history of velocities, via convolution integral over the kernel.

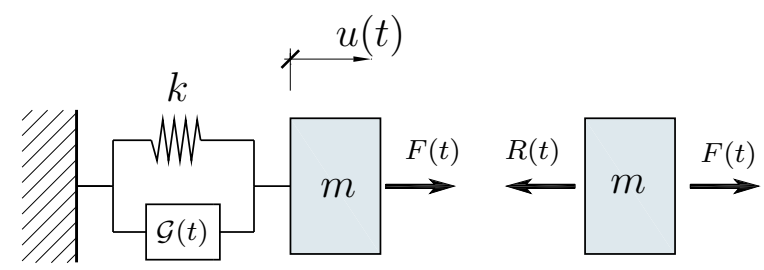

Figure 4.1: The Single-Degree-of-Freedom model with viscoelastic behavior

The motion equation can be deduced from the dynamic equilibrium

$$
\begin{aligned}
& m \ddot{u}+\int_{-\infty}^{t} \mathcal{G}(t-\tau) \dot{u}(\tau) \mathrm{d} \tau+k u(t)=F(t) \\
& u(0)=u_{0}, \dot{u}(0)=v_{0}
\end{aligned}
$$

where $u_{0}$ and $v_{0}$ are the initial position and velocity of the mass and $F(t)$ is the external, time dependent, applied force.

With reference to the mathematical form of $\mathcal{G}(t)$, several works have been published, proposing different expressions basically in the Laplace domain. The most popular is due to Biot [7] and called multi-exponential model, which assumes that the viscoelastic function is a linear combination of exponential functions. Also, the models based on the fractional derivative proposed by Bagley and Torvik [15, 16] have been widely used. Other viscoelastic models of special interest, again based on state-space methods, are the GHM approach of Golla, Hughes and McTavish [14, 40] or the Anelastic Displacement Field of Lesieutre and Mingori [41]. The reference [14] gave the required conditions of $\mathcal{G}(t)$ to describe a real dissipative motion. Adhikari and Woodhouse [43, 78] proposed viscoelastic functions in the context of damping identification in dynamic systems.

The main objective of the present work is to propose a numerical method to solve the eigenvalue problem associated with the Single-Degree-of-Freedom (SDOF) 
system described by Eq. (4.2). As known, the approximation not only embraces physical systems with only one degree of freedom, but also Multi-Degree-ofFreedom (MDOF) systems with proportional nature, i.e., those in which the dynamic matrices become diagonal in the modal space. The Laplace transform of the free-motion equation leads to a nonlinear eigenvalue problem in the Laplace variable $s$. The form of this problem will depend on the nature of the viscoelastic function transform, named $G(s)=\mathcal{L}\{\mathcal{G}(t)\}$. Several methods to solve the general nonlinear eigenvalue problem exist in the bibliography. Yang [46] and Singh [47] proposed some based on Taylor series expansion of the transcendental matrices combined with Newton's eigenvalue iteration method. Williams and Kennedy [48] developed a numerical procedure based on the parabolic interpolation of the determinant that governs the eigenvalue problem. Daya and Potier-Ferry [49] used asymptotic numerical techniques to determine the natural frequencies and the loss factors of viscoelastic damped sandwich structures. Voss [51, 52] proposed two methods based on the shift-and-invert Arnoldi's technique and on the JacobiDavidson method, respectively. In both references, an iterative process is used to compute a few eigenvalues that lay close to a given point. An important subset of viscoelastic models, in which $G(s)$ has a rational expression, have been published. In them, the nonlinear eigenvalue problem can be transformed into a linear one, introducing new internal variables and using state-space approaches, see for instance the works of Muravyov and Menon [54-56]. However, these methods become computationally expensive for large systems. In addition, the physical insight of the problem can also be lost due to the introduction of the new variables. Recently, Adhikari and Pascual [31, 32] have published efficient iterative methods to solve the eigenvalue problem for SDOF, and proportional damping systems based on the Taylor series expansion of $G(s)$.

Several authors have studied the eigensolutions as functions of the parameters involved in the dynamic matrices. The main results are related to numerical methods for the computation of eigenvalues and eigenvector derivatives respect to certain design parameter. Fox and Kapoor [79] published an important work in which analytical solutions of the derivatives were given. However, in their results the complete set of eigenvectors of the modal space are involved, and again the computations become expensive for large-order systems. Nelson [80] gave an alternative and efficient method to obtain the derivatives requiring only the eigenvector and eigenvalue of interest. Wang [81] and later Zhang and Zerva [82] introduced improved methods in truncated systems. Murthy and Haftka [83] published a survey of methods for the calculation of the derivatives applied to general nonHermitian matrix problems. The same authors in [84] studied the computational efficiency of different approximations based on eigenvalue derivatives, generalized Rayleigh quotient and the trace theorem. The generalization of the eigensolution derivatives for non-viscous systems was studied by Adhikari [59, 85]. Cortés and Elejabarrieta [60, 61] used Adhikari's solutions in an iterative numerical method 
to compute eigensolutions, applicable even for highly damped systems.

The current paper is aimed to numerically compute the eigenvalues of SDOF viscoelastic systems. As known, a dynamic model is governed by $m, k$, and several damping parameters that are included in the viscoelastic function. The newly developed eigenvalues will be functions of a single damping parameter, called valid parameter, leaving the others fixed. The key idea is to transform the characteristic equation into a differential one with separated variables. The methodology is applied to widely used viscoelastic systems and also illustrated with numerical examples to validate the theoretical results and to show the limits of application.

\subsection{Dynamics of Single Degree-of-Freedom Viscoelastic Systems}

The characteristic equation of the eigenvalue problem can be obtained trying solutions with the form $u(t)=u_{0} e^{s t}$ in the free-motion equation obtained from Eq. (4.2).

$$
m s^{2}+s G(s)+k=0
$$

Here, as mentioned, $G(s)$ is the Laplace transform of the kernel function $\mathcal{G}(t)$. Note that the eigenvalue problem in MDOF systems with proportional damping can be reduced to a set of SDOF problems, therefore, the method described in this paper can also be applied to these MDOF dynamic systems. Adhikari [29] gave the necessary and sufficient conditions under which non-viscous systems have proportional damping. The previous equation can be mass-normalized to

$$
D(s)=s^{2}+s \Gamma(s)+\omega_{n}^{2}=0
$$

The damping function $\Gamma(s)=G(s) / m$, and the natural frequency of the undamped system $\omega_{n}=\sqrt{k / m}$ have been introduced. Golla and Hughes [14] gave the necessary conditions that $G(s)$ has to fulfil in order to define a real dissipative motion. Assuming that $G(s)$ verifies these conditions, it can be assured [64, 74] that the characteristic Eq. (4.4) has $2+q$ eigenvalues, with $q \geq 0$. These can be arranged in a set with the form

$$
\left\{s_{0}, s_{0}^{*}, s_{1}, \cdots, s_{q}\right\}
$$

where $s_{0}, s_{0}^{*}$ are a pair of complex conjugate roots that induce oscillatory motion. The rest $\left\{s_{j}\right\}_{j=1}^{q}$ are named non-viscous eigenvalues [28], and are negative real numbers associated with over-critical damped responses.

According to Adhikari [28], if the set of eigenvalues is available, the complete solution of the integro-differential Eq. (4.2) can be expressed in terms of these 
eigenvalues in the form

$$
u(t)=\gamma_{0} a_{0}(t)+\gamma_{0}^{*} a_{0}^{*}(t)+\sum_{j=1}^{q} \gamma_{j} a_{j}(t)
$$

where the function and coefficient are

$$
\begin{aligned}
a_{j}(t) & =\int_{0}^{t} e^{s_{j}(t-\tau)}\left[F(\tau)-\mathcal{G}(\tau) u_{0}\right] \mathrm{d} \tau+m e^{s_{j} t}\left(v_{0}+s_{j} u_{0}\right) \\
\gamma_{j} & =\left[\frac{\partial D\left(s_{j}\right)}{\partial s}\right]^{-1}, \quad 0 \leq j \leq q
\end{aligned}
$$

Due to the relevance of the eigenvalues in the final solution, the availability of efficient tools for its numerical extraction is important. We propose a new method to compute the roots of Eq. (4.3), based on a parametric treatment of the characteristic equation. In the next section the theoretical foundations of the method are introduced.

\subsection{Parametric computation of the eigenvalues}

\subsubsection{Fundamentals of the method}

As explained in Sec. 4.2, the eigenvalues are the roots of the characteristic equation given by Eq. (4.4). The key idea of the method is to consider that the function $\Gamma=\Gamma\left(s, p_{1}, \ldots, p_{r}\right)$ depends not only on the variable $s$, but also on a set of damping parameters $\left\{p_{1}, \ldots, p_{r}\right\}$ that controls the viscoelastic behavior of the system.

In the general case, any eigenvalue $\lambda=\lambda\left(p_{1}, \ldots, p_{r}\right)$ can be considered as function of the damping parameters. Let us consider one of these parameters as variable, while the rest remain fixed. Without loss of generality, it can be assumed that this is $p_{1}$. Denoting as $\theta=p_{1}$, let us assume now that there exists a particular value (or initial point) of $\theta$, say $\theta_{0}$, so that the characteristic equation can be solved analytically in terms of $p_{2}, p_{3}, \ldots, p_{r}$, obtaining the related initial eigenvalue $\lambda_{0}$

$$
\lambda_{0}^{2}+\lambda_{0} \Gamma\left(\lambda_{0}, \theta_{0}, p_{2}, \ldots, p_{r}\right)+\omega_{n}^{2}=0 \rightarrow \lambda_{0}=\lambda_{0}\left(\theta_{0}, p_{2}, \ldots, p_{r}\right)
$$

Under these conditions the parameter $\theta=p_{1}$ is named valid parameter for the purposes of the current paper. Since the rest of parameters are fixed, the eigenvalue $\lambda=\lambda(\theta)$ can be considered as single-variable function of $\theta$. The conditions of existence and uniqueness of such function are given by the implicit function 
theorem. As known, the equation

$$
D(\lambda, \theta)=\lambda^{2}+\lambda \Gamma(\lambda, \theta)+\omega_{n}^{2}=0
$$

defines an implicit function $\lambda=\lambda(\theta)$ around the point $\theta=\theta_{0}$ if

$$
\frac{\partial D\left(\lambda_{0}, \theta_{0}\right)}{\partial \lambda}=\Gamma\left(\lambda_{0}, \theta_{0}\right)+\lambda_{0}\left[2+\frac{\partial \Gamma\left(\lambda_{0}, \theta_{0}\right)}{\partial \lambda}\right] \neq 0
$$

Under this hypothesis, it is also guaranteed that there exist: (i) an open interval $I=] \theta_{0}-h / 2, \theta_{0}+h / 2[$ with $h>0$, and (ii) an open neighborhood around the point $\lambda_{0}$ in the complex domain $B \subset \mathbb{C}$, such that the function $\lambda(\theta)$ will be unique and continuously differentiable in $I$ and its graph verifies

$$
\{(\theta, \lambda(\theta)): \theta \in I\}=\{(s, \theta) \in B \times I: D(s, \theta)=0\}
$$

Notice that the damping function has been represented directly as $\Gamma(s, \theta)$ in the implicit function theorem, due to the fact that the parameters $p_{2}, \ldots, p_{r}$ are assumed fixed. The main challenge of this paper is to construct approximated solutions of the function $\lambda(\theta)$. In the next subsection the methodology to obtain its $k$ th-order Taylor series will be developed. Moreover, this approximation will be used in a further step to improve the solution.

\subsubsection{Taylor expansion of the eigenvalues}

Assuming that the necessary conditions for the existence of the function $\lambda=\lambda(\theta)$ are satisfied, the Taylor series expansion up to the $k$ th order can be obtained around the initial point $\theta_{0}$, provided that the derivatives evaluated at this point are available.

$$
\lambda_{k}(\theta)=\lambda_{0}+\sum_{r=1}^{k} \frac{\lambda^{(r)}\left(\theta_{0}\right)}{r !}\left(\theta-\theta_{0}\right)^{r}
$$

with

$$
\lambda^{(r)}\left(\theta_{0}\right)=\left.\frac{\mathrm{d}^{r} \lambda}{\mathrm{d} \theta^{r}}\right|_{\theta=\theta_{0}}
$$

The computation of $\lambda^{(r)}\left(\theta_{0}\right)$ can be carried out from the characteristic Eq. (4.9), in which consecutive derivatives can be taken respect to $\theta$. The first order derivative is calculated as

$$
\frac{\mathrm{d} D}{\mathrm{~d} \theta}=2 \lambda \lambda^{\prime}+\lambda^{\prime} \Gamma+\lambda\left(\Gamma_{\lambda}^{\prime} \lambda^{\prime}+\Gamma_{\theta}^{\prime}\right)=0
$$

whence solving $\lambda^{\prime}$ results in

$$
\lambda^{\prime}=\frac{-\lambda \Gamma_{\theta}^{\prime}}{\Gamma+\lambda\left[2+\Gamma_{\lambda}^{\prime}\right]}
$$


and

$$
\Gamma_{\lambda}^{\prime}=\frac{\partial \Gamma}{\partial \lambda}, \quad \Gamma_{\theta}^{\prime}=\frac{\partial \Gamma}{\partial \theta}
$$

Using the same procedure to compute the second derivative, the term $\lambda^{\prime \prime}$ is extracted. After some simplifications, this second derivative results in

$$
\lambda^{\prime \prime}=-\frac{R\left(\theta, \lambda, \lambda^{\prime}\right)}{\left(\Gamma+\lambda\left[2+\Gamma_{\lambda}^{\prime}\right]\right)^{2}}
$$

where

$$
R\left(\theta, \lambda, \lambda^{\prime}\right)=\left(\lambda^{\prime}\right)^{2}\left(2+2 \Gamma_{\lambda}^{\prime}+\lambda \Gamma_{\lambda \lambda}^{\prime \prime}\right)+2 \lambda^{\prime}\left(\Gamma_{\theta}^{\prime}+\lambda \Gamma_{\lambda \theta}^{\prime \prime}\right)+\lambda \Gamma_{\theta \theta}^{\prime \prime}
$$

and now

$$
\Gamma_{\lambda \lambda}^{\prime \prime}=\frac{\partial^{2} \Gamma}{\partial \lambda^{2}}, \quad \Gamma_{\lambda \theta}^{\prime \prime}=\frac{\partial^{2} \Gamma}{\partial \lambda \partial \theta}, \quad \Gamma_{\theta \theta}^{\prime \prime}=\frac{\partial^{2} \Gamma}{\partial \theta^{2}}
$$

To find more terms of the series would be possible if higher order derivatives were calculated. However, for the proposes of the present work, only the explicit expressions of the first two derivatives are necessary. Note that both are well defined since the denominators of Eqs. (4.15), (4.17) are never nil due to Eq. (4.10).

As will later be described, in some cases Eq. (4.12) suffices when the objective is to calculate the eigenvalue at the proximities of point $\theta_{0}$. However, the availability of the solution in a wider interval of the parameter $\theta$ is sometimes necessary. For these situations, an improved solution is presented in the next subsection.

\subsubsection{Improving the solution. Eigenvalue differential equation}

The challenge now is to use the previously obtained results to improve the estimation accuracy of $\lambda(\theta)$ in a wider range of $\theta$. To describe the procedure, the expression of the first order derivative $\lambda^{\prime}(\theta)$ given in Eq. (4.15) is rewritten in the form

$$
\lambda^{\prime}=-\lambda \Psi(\lambda, \theta)
$$

where the new function $\Psi(\lambda, \theta)$ is defined by

$$
\Psi(\lambda, \theta)=\frac{\Gamma_{\theta}^{\prime}(\lambda, \theta)}{\Gamma(\lambda, \theta)+\lambda\left[2+\Gamma_{\lambda}^{\prime}(\lambda, \theta)\right]}
$$

From a mathematical point of view, Eq. (4.20) can be considered an ordinary differential equation to be complemented with the initial condition $\lambda_{0}=\lambda\left(\theta_{0}\right)$. 
Obviously, its solution is the same as that of the characteristic Eq. (4.8). The fundamental objective of the current method is to transform this ordinary differential equation into another with separated variables. For that, the $k$ th order solution of the Taylor series obtained in Eq. (4.12), namely $\lambda_{k}(\theta)$, will be used to approximate the value of $\lambda$ inside the function $\Psi(\lambda, \theta)$. So

$$
\Psi(\lambda, \theta) \approx \Psi\left(\lambda_{k}(\theta), \theta\right) \equiv \psi_{k}(\theta)
$$

Hence, the new function $\psi_{k}(\theta)$ depends only on a single variable. The accuracy of the approximation is related to the quality of the assumption made in Eq. (4.22), that in turn is conditioned by the level of viscoelasticity of the system. In other words, depends on the variability of the viscoelastic function $\Gamma(s, \theta)$ with respect to $s$; a viscoelastic system with low viscoelasticity does not present large variations in the $s$-domain. More details about the study of the viscoelasticity in non-viscous dynamic systems can be found in the work of Adhikari and Woodhouse [71]. Here it will be shown that, under certain conditions, the approximation $\Psi(\lambda, \theta) \approx \Psi\left(\lambda_{k}(\theta), \theta\right)$ will produce results close to the exact ones. With regard to the order of the approximation, in the next subsection it will be demonstrated that the proposed method improves the error order respect to that of the Taylor approximation. However, as will be shown with the numerical examples, the order does not ensure a better mean-approximation in the wider range of the parameter.

Under the aforementioned assumptions, the differential equation can be expressed as

$$
\frac{\lambda^{\prime}}{\lambda}=-\psi_{k}(\theta), \quad \lambda\left(\theta_{0}\right)=\lambda_{0}
$$

whose solution will be denoted by $\hat{\lambda}_{k}(\theta)$. The subindex indicates the $k$-order Taylor series approximation. An explicit expression can be represented by

$$
\hat{\lambda}_{k}(\theta)=\lambda_{0} e^{-Z(\theta)}
$$

where the exponential function is defined by

$$
Z(\theta)=\int_{\theta_{0}}^{\theta} \psi_{k}(\vartheta) \mathrm{d} \vartheta
$$

When the function $\hat{\lambda}_{k}(\theta)$ is obtained from the first order approximation of the Taylor series expansion $k=1$, the solution will be named Improved Linear Solution (ILS). If the second order approximation is used $k=2$, the name will be Improved Quadratic Solution (IQS). The functions $\Psi(s, \theta)$ and $\psi_{k}(\theta)$ will be called exact and approximated integrand function, respectively. The next subsection is aimed at the analysis of the truncation error. 


\subsection{Analysis of the error}

Sec. 4.3 was focused on the methodology exposition to compute the function $\hat{\lambda}_{k}(\theta)$ as approximation of the exact eigenvalues $\lambda(\theta)$. It has already been mentioned that intuitively, the proposed function will be a good estimation provided that the error between the functions $\Psi(\lambda(\theta), \theta)$ and $\psi_{k}(\theta)$ is not important. In this subsection more quantitative information is provided, finding a bound of the error $\left|\hat{\lambda}_{k}(\theta)-\lambda(\theta)\right|$. The necessary conditions to calculate this bound are directly related with the properties of the viscoelastic function $G(s, \theta)=m \Gamma(s, \theta)$ and will be given by three hypotheses:

H1 If $\left(\lambda_{0}, \theta_{0}\right)$ is the initial point verifying Eq. (4.8), then $\Gamma\left(\lambda_{0}, \theta_{0}\right)+\lambda_{0}[2+$ $\left.\Gamma_{\lambda}^{\prime}\left(\lambda_{0}, \theta_{0}\right)\right] \neq 0$

H2 Let $I, B$ be the neighborhoods of $\theta_{0}, \lambda_{0}$, respectively; the existence of these sets is assured by the implicit function theorem. Then $G(s, \theta)$ is a continuous and differentiable function respect to the variables $s, \theta$ up to order $k+1$ in the set $B \times I$. In addition, $G(s, \theta)$ and its partial derivatives respect to $s, \theta$ up to order $k+1$ are bounded in the set $B$

H3 The function $\Psi(s, \theta)$ defined by Eq. (4.21) is Lipschitz continuous respect to the first variable $s$ in the set $B$, with constant $q>0$

$$
\left|\Psi\left(s_{1}, \theta\right)-\Psi\left(s_{2}, \theta\right)\right| \leq q\left|s_{1}-s_{2}\right| \quad \forall s_{1}, s_{2} \in B
$$

The hypothesis $\mathrm{H} 1$ allows to assure the existence of the function $\lambda(\theta): I \rightarrow B$ in the interval $I=] \theta_{0}-h / 2, \theta_{0}+h / 2[$. One of the thesis of the implicit function theorem is the existence of the set $B \subset \mathbb{C}$ in which the image of $\lambda(\theta)$ is defined. Under this considerations, hypotheses $\mathrm{H} 2, \mathrm{H} 3$ are well stablished because they are based on the existence of such sets. The main conclusions of the current subsection will be presented in Theorem 1. Previously, two Lemmas are formulated since they will be necessary later in the demonstration of the main theorem.

Lemma 1. If $\Psi(s, \theta)$ is a function from $E q$. (4.21), then a real and positive number exists, $p>0$, such that $|\Psi(s, \theta)| \leq p \quad \forall(s, \theta) \in B \times I$

Lemma 2. Let $\lambda(\theta)$ be the eigenvalue as function of the parameter $\theta \in I$. Then for any integer $k \geq 0$ there exists a real and positive number, $\rho_{k}>0$, such that $\left|\lambda^{(k)}(\theta)\right| \leq \rho_{k} \quad \forall \theta \in I$

Notice that with the previous nomenclature $\lambda^{(0)}(\theta)=\lambda(\theta)$. The proof of Lemmas 1 and 2 are found in Appendices 4.6 and 4.6 
Theorem 1. Let $\lambda(\theta)$ be the eigenvalue and $\hat{\lambda}_{k}(\theta)$ the approximation calculated from Eqs. (4.22) to (4.25) with $k \geq 1$. Assuming that hypotheses H1, H2 and H3 are satisfied, there exist two positive real numbers, $\rho_{k}>0, p>0$ such that

$$
\left|\hat{\lambda}_{k}(\theta)-\lambda(\theta)\right| \leq q\left|\lambda_{0}\right| \frac{\rho_{k}}{(k+1) !} h^{k+2} e^{p h} \quad \forall \theta \in I
$$

Proof. From Eq. (4.20), the exact eigenvalue $\lambda(\theta)$ verifies the relation

$$
\lambda(\theta)=\lambda_{0} e^{-Y(\theta)}
$$

where

$$
Y(\theta)=\int_{\theta_{0}}^{\theta} \Psi(\lambda(\vartheta), \vartheta) \mathrm{d} \vartheta
$$

For demonstration purposes, it is necessary to bound the difference norm $\mid Z^{n}(\theta)-$ $Y^{n}(\theta) \mid$ for any natural number $n \geq 1$.

First, using H3 and the subtraction $\hat{\lambda}_{k}(\theta)-\lambda(\theta)$ in Taylor series, the previous norm for $n=1$ is

$$
\begin{aligned}
|Z(\theta)-Y(\theta)| & \leq \int_{\theta_{0}}^{\theta}\left|\Psi\left(\lambda_{k}(\theta), \theta\right)-\Psi(\lambda(\theta), \theta)\right| \mathrm{d} \vartheta \\
& \leq \int_{\theta_{0}}^{\theta} q\left|\lambda_{k}(\theta)-\lambda(\theta)\right| \mathrm{d} \vartheta \leq q \int_{\theta_{0}}^{\theta} \frac{\left|\lambda^{(k+1)}(\xi)\right|}{(k+1) !}\left|\xi-\theta_{0}\right|^{k+1} \mathrm{~d} \vartheta \\
& \leq q \frac{\left|\lambda^{(k+1)}(\xi)\right|}{(k+1) !} h^{k+2} \leq \frac{q \rho_{k}}{(k+1) !} h^{k+2}
\end{aligned}
$$

where $\xi \in I$ defines the truncation residual of the Taylor series up to the order $k$. For the last inequality Lemma 2 has been used. In general, a bound of the functions $Z(\theta), Y(\theta)$ can be calculated using Lemma 1. From the definitions, one directly obtains

$$
\begin{aligned}
|Z(\theta)| & \leq \int_{\theta_{0}}^{\theta}\left|\Psi\left(\lambda_{k}(\theta), \theta\right)\right| \mathrm{d} \vartheta \leq p h \\
|Y(\theta)| & \leq \int_{\theta_{0}}^{\theta}|\Psi(\lambda(\theta), \theta)| \mathrm{d} \vartheta \leq p h
\end{aligned}
$$


Second, using Eqs. (4.29), (4.30) for $n \geq 1$

$$
\begin{aligned}
\left|Z^{n}(\theta)-Y^{n}(\theta)\right| & =\left|[Z(\theta)-Y(\theta)] \sum_{j=0}^{n} Z^{n-j}(\theta) Y^{j-1}(\theta)\right| \\
& \leq|Z(\theta)-Y(\theta)| \sum_{j=1}^{n}|Z(\theta)|^{n-j}|Y(\theta)|^{j-1} \\
& \leq|Z(\theta)-Y(\theta)| \sum_{j=1}^{n}(p h)^{n-j}(p h)^{j-1} \\
& =|Z(\theta)-Y(\theta)| n(p h)^{n-1}
\end{aligned}
$$

Now the error can be calculated; using the definitions of both functions

$$
\begin{aligned}
\left|\hat{\lambda}_{k}(\theta)-\lambda(\theta)\right| & =\left|\lambda_{0}\right|\left|\sum_{n=1}^{\infty} \frac{(-1)^{n}}{n !}\left[Z^{n}(\theta)-Y^{n}(\theta)\right]\right| \\
& \leq\left|\lambda_{0}\right| \sum_{n=1}^{\infty} \frac{1}{n !}\left|Z^{n}(\theta)-Y^{n}(\theta)\right| \\
& \leq\left|\lambda_{0}\right||Z(\theta)-Y(\theta)| \sum_{n=1}^{\infty} \frac{n(p h)^{n-1}}{n !} \\
& \leq\left|\lambda_{0}\right||Z(\theta)-Y(\theta)| \sum_{n=1}^{\infty} \frac{(p h)^{n-1}}{(n-1) !} \\
& \leq\left|\lambda_{0}\right||Z(\theta)-Y(\theta)| e^{p h} \\
& \leq q\left|\lambda_{0}\right| \frac{\rho_{k}}{(k+1) !} h^{k+2} e^{p h}
\end{aligned}
$$

The theorem states that the approximation order of the improved solution is $\mathcal{O}\left(h^{k+2}\right)$, while approximating with Taylor series up to the $k$ th term gives $\mathcal{O}\left(h^{k+1}\right)$. This result allows us to assure that an improvement of the solution in a neighborhood of the initial point exists.

To illustrate the efficiency of the proposed method, the next Section describes its implementation for systems with a Biot's model-based viscoelastic function. Cases with one exponential kernel and with more than one will be also developed. In addition, the viscously damping systems are analyzed, since the current methodology allows to find analytical solutions. 


\subsection{Analysis of particular cases}

\subsubsection{The viscous model}

The viscous model is a special case of viscoelastic behavior whose kernel is proportional to the Dirac delta, $\mathcal{G}(t)=c_{v} \delta(t)$, where $c_{v}$ is the viscous damping coefficient. The Laplace transform of the kernel function becomes the $s$-independent function $G(s) \equiv c_{v}$. In general, for SDOF systems instead of $c_{v}$ the damping ratio $\zeta$ is commonly used. Both are related by the well known expression $c_{v}=2 m \omega_{n} \zeta$, and consequently the mathematical model depends only on the single parameter $\theta \equiv \zeta$. The mass-normalized characteristic Eq. (4.3) can be rewritten as

$$
s^{2}+2 s \zeta \omega_{n}+\omega_{n}^{2}=0
$$

where now $\Gamma(s, \zeta)=2 \zeta \omega_{n}$. The classical exact solution of Eq. (4.33) is the pair of complex numbers

$$
\lambda(\zeta)=-\omega_{n} \zeta \pm i \omega_{n} \sqrt{1-\zeta^{2}}
$$

Although the exact solution is here available, the application of the proposed method is of interest, due to the availability of analytical solutions for Eq. (4.23) considering Taylor series up to the linear order $\lambda_{1}(\zeta)$ or up to the quadratic one $\lambda_{2}(\zeta)$.

Taking $\zeta_{0}=0$ as the initial point, the solution of the characteristic equation corresponds to that of the undamped system, $\lambda(0)=\lambda_{0}= \pm i \omega_{n}$. Using now the eigenvalue with positive imaginary part, the derivatives of $\lambda(\zeta)$ can be deduced from Eqs. (4.15), (4.17)

$$
\begin{array}{rll}
\lambda^{\prime}(\zeta)=-\frac{\omega_{n} \lambda(\zeta)}{\omega_{n} \zeta+\lambda(\zeta)} & \rightarrow & \lambda^{\prime}(0)=-\omega_{n} \\
\lambda^{\prime \prime}(\zeta)=-\lambda^{\prime}(\zeta) \frac{2 \omega_{n}+\lambda^{\prime}(\zeta)}{\omega_{n} \zeta+\lambda(\zeta)} & \rightarrow & \lambda^{\prime \prime}(0)=-i \omega_{n}
\end{array}
$$

The Taylor series expansion of the function $\lambda(\zeta)$ up to the second order can be obtained as

$$
\lambda_{2}(\zeta)=i \omega_{n}-\omega_{n} \zeta-\frac{i \omega_{n} \zeta^{2}}{2} \equiv \lambda_{1}(\zeta)-\frac{i \omega_{n} \zeta^{2}}{2}
$$

It can be verified that the three first terms of the Taylor expansion (Eq. (4.34)) coincide with Eq. (4.36). In view of the theoretical results of the previous section, the improved solutions ILS $(k=1)$ and IQS $(k=2)$ can be computed solving the following differential equation

$$
\lambda^{\prime}=-\lambda \frac{\omega_{n}}{\omega_{n} \zeta+\lambda_{k}(\zeta)}, \quad \lambda(0)=i \omega_{n}
$$


Substituting in Eq. (4.37) the first order approximation of the root $\lambda_{1}(\zeta)$ from Eq. (4.36), the ordinary differential equation leads to

$$
\lambda^{\prime}=i \lambda, \quad \lambda(0)=i \omega_{n}
$$

and a closed expression for ILS is obtained

$$
\hat{\lambda}_{1}(\zeta)=i \omega_{n} e^{i \zeta}
$$

It is also interesting to deduce the improved solution corresponding to the quadratic approximation $\lambda_{2}(\zeta)$ from Eq. (4.36); the differential equation results in

$$
\lambda^{\prime}=\lambda \frac{i}{1-\zeta^{2} / 2}, \quad \lambda(0)=i \omega_{n}
$$

and the explicit expression of IQS can also be easily integrated, resulting in

$$
\hat{\lambda}_{2}(\zeta)=i \omega_{n}\left(\frac{\sqrt{2}+\zeta}{\sqrt{2}-\zeta}\right)^{\frac{i}{\sqrt{2}}}
$$

Both improved solutions together with the exact one are plotted vs. the damping ratio $\zeta$ in Fig. 4.2. This ratio is represented in logarithmic scale since the great majority of dynamic systems are lightly damped, with range $0.001 \leq \zeta \leq 0.2$. For this numerical example an undamped natural frequency $\omega_{n}=1 \mathrm{rad} / \mathrm{s}$ has been chosen. The improved solutions are close or very close to the exact one through almost the complete range of $\zeta$, even for high damped systems. The noticeable differences are for $\zeta>0.6$, far from the initial value $\zeta_{0}=0$ as mentioned at the end of Sec. 4.4.

Moreover, it can be noted that the improved solutions and the exact one lay along the same complex domain circumference when the parameter $\zeta$ varies. This fact can easily be verified calculating the absolute value of Eqs. (4.39), (4.41)

$$
\left|\hat{\lambda}_{1}(\zeta)\right|=\left|\hat{\lambda}_{2}(\zeta)\right|=|\lambda(\zeta)|=\omega_{n}, \quad \forall \zeta \geq 0
$$

that coincides with the absolute value of the exact root for any damping ratio.

\subsubsection{The Biot's exponential model}

This model was introduced by Biot [7] and has been extensively used for the analysis of viscoelastic systems. It is based on the assumption that dissipative forces depend on the history of the velocities of the degrees of freedom via an exponential kernel with form

$$
\mathcal{G}(t)=c_{v} \mu e^{-\mu t}, \quad \mu, t \geq 0
$$




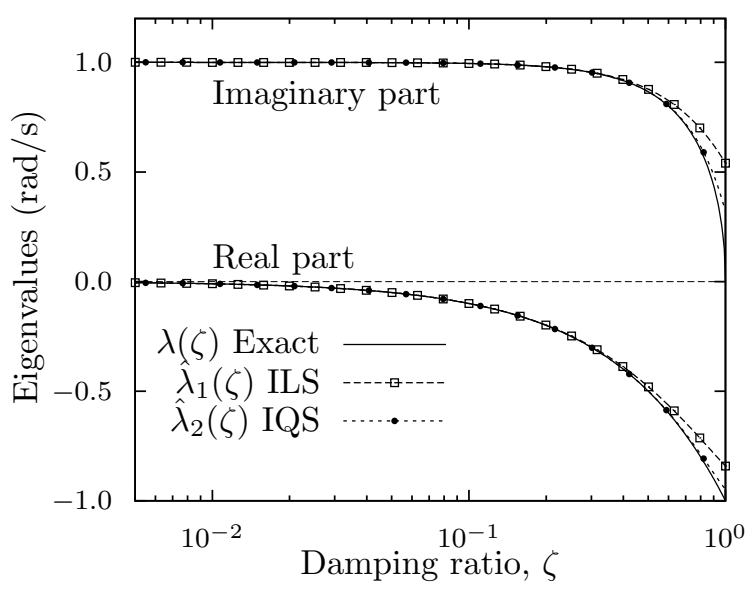

Figure 4.2: Eigenvalues of viscous model vs. $\zeta$. Continuous line for exact values and dotted for Improved Solutions; square tick for Linear (ILS), bullet for Quadratic (IQS).

where $\mu$ is the relaxation parameter, also called non-viscous parameter. Notice that for high values of $\mu$ the viscoelastic model tends to the viscous model, mathematically expressed by $\lim _{\mu \rightarrow \infty} \mathcal{G}(t)=c_{v} \delta(t)$. Therefore, the non-viscous behavior will be controlled by $\mu$, so that when $\mu>>\omega_{n}$ the damping model is viscous with coefficient $c_{v}[28,71]$. Otherwise, when $\mu<<\omega_{n}$ the behavior is highly nonviscous. The characteristic equation in the Laplace domain can be written as

$$
m s^{2}+s G(s)+k=0
$$

where $G(s)=c_{v} \mu /(s+\mu)$. The coefficient of the limit viscous model can again be expressed in terms of the damping ratio $c_{v}=2 m \omega_{n} \zeta$. Under these conditions, the viscoelastic model can be written as function of the two parameters, $\zeta, \mu$ as follows

$$
\lambda^{2}+2 \omega_{n} \zeta \frac{\mu}{\lambda+\mu} \lambda+\omega_{n}^{2}=0
$$

Introducing a new parameter $\tau=1 / \mu$ with $\mu>0$, the previous characteristic equation can be rewritten as

$$
\lambda^{2}+2 \omega_{n} \zeta \frac{1}{\tau \lambda+1} \lambda+\omega_{n}^{2}=0
$$

Eqs. (4.45), (4.46) allow to define the eigenvalues $\lambda=\lambda(\theta)$ as single-variable functions of either $\theta=\mu, \theta=\tau$ or of $\theta=\zeta$. Table 4.1 shows information related 
with the three cases and the eigenvalues evaluated at the initial values $\left(\lambda_{0}, \theta_{0}\right)$. In the present subsection the first two cases will be developed. The third one will be considered for a model with $N$ exponential kernels in subsection 4.5.3.

\begin{tabular}{lrrr}
\hline Parameter, $\theta$ & $\theta=\mu$ & $\theta=\tau$ & $\theta=\zeta$ \\
\hline Function $\lambda(\theta)$ & $\lambda(\mu)$ & $\lambda(\tau)$ & $\lambda(\zeta)$ \\
Initial par., $\theta_{0}$ & $\mu_{0}=0$ & $\tau_{0}=0$ & $\zeta_{0}=0$ \\
Initial eig., $\lambda_{0}=\lambda\left(\theta_{0}\right)$ & $\pm i \omega_{n}$ & $-\omega_{n} \zeta \pm i \omega_{n} \sqrt{1-\zeta^{2}}$ & $\pm i \omega_{n}$ \\
Subseccion & $4.5 .2($ Case 1$)$ & 4.5 .2 (Case 2) & 4.5 .3 \\
$D(\lambda, \theta)$ & Eq. 4.47 & Eq. 4.52 & Eq. 4.59 \\
Results & Figs. 4.3-4.5 & Figs. 4.6-4.8 & Figs. 4.9-4.11 \\
\hline
\end{tabular}

Table 4.1: Biot's exponential model. Damping parameters, considered eigenvalue functions and initial values of the proposed method.

Case 1, $\theta=\mu$. The function $\lambda(\mu)$ is implicitly defined by

$$
D(\lambda, \mu)=\lambda^{2}+2 \omega_{n} \zeta \frac{\mu}{\lambda+\mu} \lambda+\omega_{n}^{2}=0
$$

As an initial point, any root of the equation $D(\lambda, 0)=\lambda^{2}+\omega_{n}^{2}=0$ can be taken. Without loss of generality we will use the positive imaginary part $\lambda_{0}=i \omega$. From Eqs. (4.15), (4.17) the values of $\lambda^{\prime}(0), \lambda^{\prime \prime}(0)$ can be computed, obtaining after some operations the expressions

$$
\lambda^{\prime}(0)=i \zeta, \quad \lambda^{\prime \prime}(0)=-\frac{\zeta(2+\zeta)}{\omega_{n}}
$$

Hence, the second order Taylor polynomial is

$$
\lambda_{2}(\mu)=i \omega_{n}+i \zeta \mu-\frac{\zeta(2+\zeta)}{2 \omega_{n}} \mu^{2}, \quad \mu \geq 0
$$

The improved solutions ILS and IQS constructed from linear and quadratic Taylor approximations respectively, can be calculated with Eq. (4.24). For that, the expression of the approximated integrand function must be considered

$$
\psi_{k}(\mu)=\frac{\lambda_{k}(\mu) \zeta \omega_{n}}{\lambda_{k}(\mu)\left[\lambda_{k}(\mu)+\mu\right]^{2}+\zeta \mu^{2} \omega_{n}}
$$

where $\lambda_{k}(\mu)$ is defined by Eq. (4.12). As described before, the quality of the approximated eigenvalue function $\hat{\lambda}_{k}(\mu)$, depends on the accuracy of the assumption over the integrand function $\Psi(\lambda(\mu), \mu) \approx \psi_{k}(\mu)$ given by Eq. (4.22). The method can be implemented calculating the integral given in Eq. (4.25) through 
numerical quadrature for each parameter value. The results have been represented in Figs. 4.3-4.5, where the functions $\hat{\lambda}_{1}(\mu), \hat{\lambda}_{2}(\mu)$ have been plotted vs. $\mu$ for a SDOF system with natural frequency $\omega_{n}=1 \mathrm{rad} / \mathrm{s}$. Three damping ratios $\zeta=\{0.01,0.05,0.10\}$ have been considered for comparison. In addition, the relative error of the exact integrand function $\Psi(\lambda, \mu)$

$$
\epsilon_{k}(\mu)=\left|\frac{\Psi(\lambda(\mu), \mu)-\psi_{k}(\mu)}{\Psi(\lambda(\mu), \mu)}\right|
$$

is shown. Notice that the exact eigenvalue $\lambda(\mu)$ from Eq. (4.45) is required for the error computation.

Figs. 4.3-4.5 shows that as expected, close to the initial point $\mu=0$ the proposed method eigenvalues are very accurate for all damping levels. Moreover, it can be seen that $\epsilon_{2}(\mu)<\epsilon_{1}(\mu)$ in the range $0<\mu<1$, inequality that as predicted from the theory of Sec. 4.4, implies that IQS is more precise than ILS. On the contrary, when $\mu>1$ the errors are $\epsilon_{1}(\mu)<\epsilon_{2}(\mu)$ and in addition, while $\epsilon_{2}(\mu)$ increases monotonically, $\epsilon_{1}(\mu)$ remains almost constant. Therefore in this range ILS is more accurate and stable than IQS so that the eigenvalues calculated with ILS are in average closer to the exact solution over the considered range of $\mu$. For a fixed $\mu$, the higher the damping the poorer the approximation, specially for IQS that completely fails for high values of $\mu$ and $\zeta$. The divergence of IQS in this situations can be avoided with a different choice of the parameter as will be explained in the next case.

This example suggests that the improved solution with quadratic approximation is not always the best option when the results are required in a wide range.

Case 2, $\theta=\tau=1 / \mu$. The solution of this case is somewhat equivalent to the expansion of the eigenvalues in their Taylor series around $\mu \rightarrow \infty$, or $\tau=0$. As will be seen, the use of this parameter results in a significant improvement of the results from those of the previous case. The function $\lambda=\lambda(\tau)$ is now implicitly defined by the equation

$$
D(\lambda, \tau)=\lambda^{2}+2 \omega_{n} \zeta \frac{1}{\tau \lambda+1} \lambda+\omega_{n}^{2}=0
$$

The complex roots of this equation for $\tau_{0}=0$ have already been presented in Table 4.1. As before, Eqs. (4.15), (4.17) are used to analytically calculate the derivatives evaluated at the initial point

$$
\begin{aligned}
\lambda^{\prime}(0) & =\frac{\zeta \lambda_{0}^{2} \omega_{n}}{\lambda_{0}+\zeta \omega_{n}} \\
\lambda^{\prime \prime}(0) & =-\zeta \lambda_{0}^{3} \omega_{n} \frac{2 \lambda_{0}^{2}+\zeta \lambda_{0} \omega_{n}-2 \zeta^{2} \omega_{n}^{2}}{\left(\lambda_{0}+\zeta \omega_{n}\right)^{3}}
\end{aligned}
$$




$$
\zeta=0.01
$$
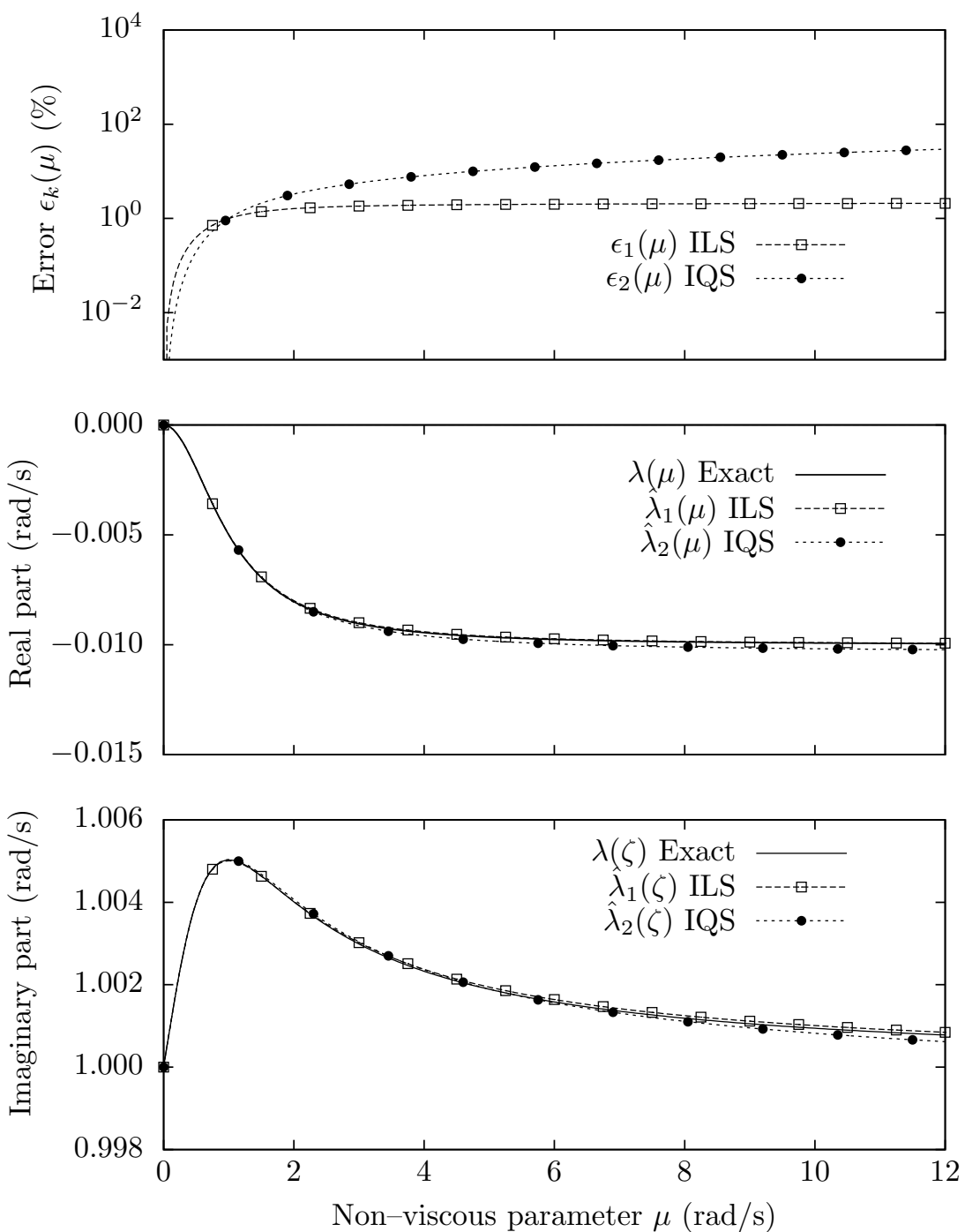

Figure 4.3: Case $\theta=\mu$. Relative error $\epsilon_{k}(\mu)=\left|\Psi-\psi_{k}\right| /|\Psi|$ of the integrand function (top). Eigenvalues vs. parameter $\mu$ (middle, bottom) for $\zeta=0.01$; continuous line for exact values and dotted for Improved Solutions; square tick for Linear (ILS), bullet for Quadratic (IQS). 


$$
\zeta=0.05
$$
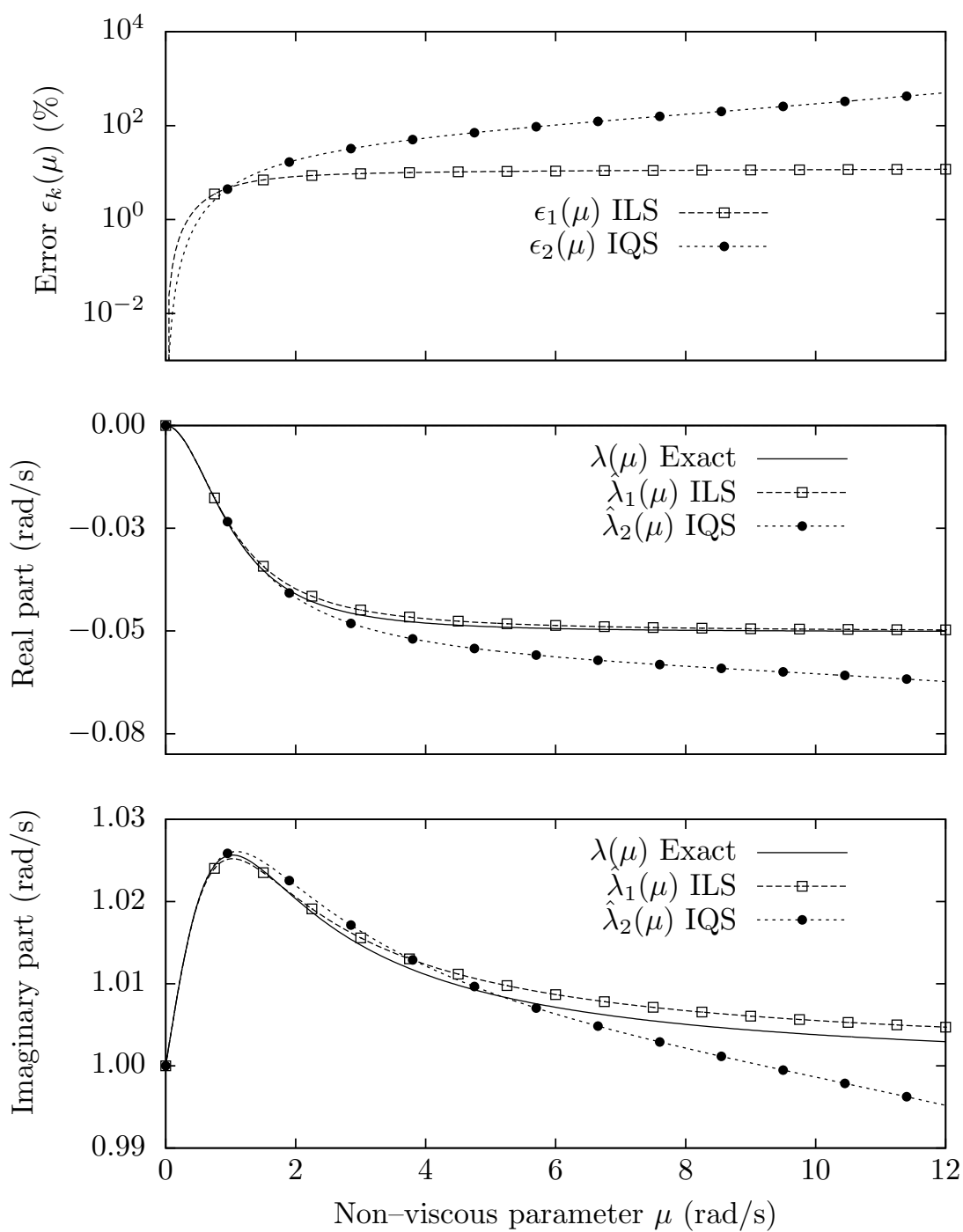

Figure 4.4: Case $\theta=\mu$. Relative error $\epsilon_{k}(\mu)=\left|\Psi-\psi_{k}\right| /|\Psi|$ of the integrand function (top). Eigenvalues vs. parameter $\mu$ (middle, bottom) for $\zeta=0,05$; continuous line for exact values and dotted for Improved Solutions; square tick for Linear (ILS), bullet for Quadratic (IQS). 


$$
\zeta=0.10
$$
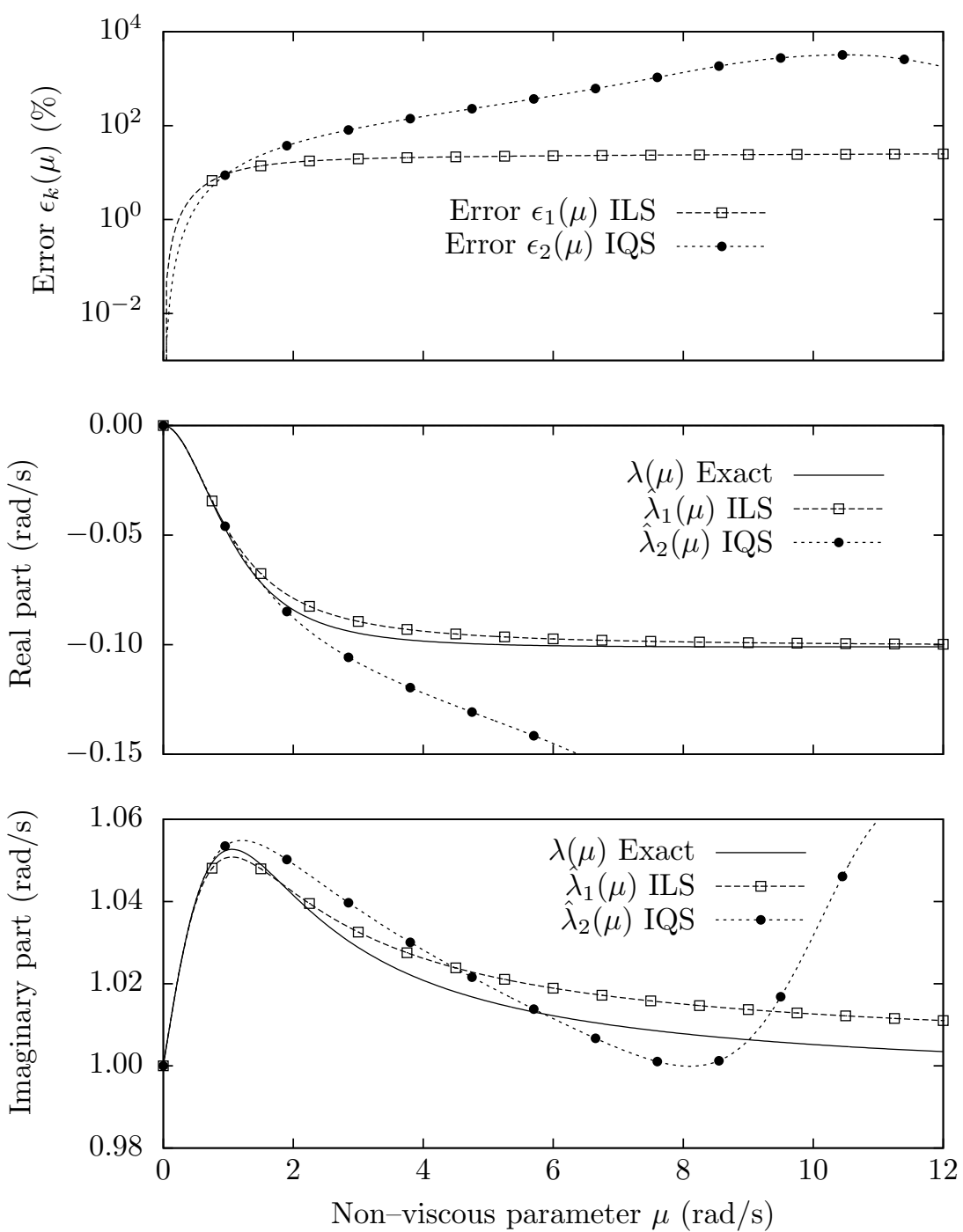

Figure 4.5: Case $\theta=\mu$. Relative error $\epsilon_{k}(\mu)=\left|\Psi-\psi_{k}\right| /|\Psi|$ of the integrand function (top). Eigenvalues vs. parameter $\mu$ (middle, bottom) for $\zeta=0.10$; continuous line for exact values and dotted for Improved Solutions; square tick for Linear (ILS), bullet for Quadratic (IQS). 
With these expressions, the second order Taylor series expansion of $\lambda(\tau)$ is

$$
\lambda_{2}(\tau)=\lambda_{0}+\frac{\zeta \lambda_{0}^{2} \omega_{n}}{\lambda_{0}+\zeta \omega_{n}} \tau-\frac{2 \lambda_{0}^{2}+\zeta \lambda_{0} \omega_{n}-2 \zeta^{2} \omega_{n}^{2}}{2\left(\lambda_{0}+\zeta \omega_{n}\right)^{3}} \zeta \lambda_{0}^{3} \omega_{n} \tau^{2}
$$

defined in the range $\zeta, \tau \geq 0$. Now, the improved solutions ILS, IQS can be calculated with the methodology described in subsection 4.3.3. First, in this particular case

$$
\psi_{k}(\tau)=-\frac{\lambda_{k}(\tau) \zeta \omega_{n}}{\lambda_{k}(\tau)\left[1+\tau \lambda_{k}(\tau)\right]^{2}+\zeta \omega_{n}}
$$

Second, for each value of the parameter $\tau$, the eigenvalue $\hat{\lambda}_{k}(\tau)=\lambda_{0} e^{-Z(\tau)}$ can be estimated by numerical quadrature of the integral

$$
Z(\tau)=\int_{0}^{\tau} \psi_{k}(t) \mathrm{d} t
$$

Figs. 4.6-4.8 show the error and the complex eigenvalues vs. $\tau$ from the exact and the two improved solutions. To compare the results with those of the previous example, the same $\mu$-axis has been used, taking the inverse of $\tau$. It is clear that the choice of $\tau$ produces better results for all $\zeta$ and for the complete range of $\mu$. The distributions of $\epsilon_{1}(\mu), \epsilon_{2}(\mu)$ reverse their relative position but now both tend to zero for high values of $\mu$. Except close to the origin $\mu=0(\tau \rightarrow \infty)$, the estimated eigenvalues are very close to the exact ones. The ILS is again the best solution, although the IQS presents now an almost perfect behavior, even for high damping.

\subsubsection{The Biot's multi-exponential model}

This model arises as a natural generalization of the Biot's single-exponential model. Using the same nomenclature as in the previous subsection (Eq. (4.43)), the viscoelastic kernel function can be written as

$$
\mathcal{G}(t)=\frac{c_{v}}{N} \sum_{j=1}^{N} \mu_{j} e^{-\mu_{j} t}, \quad \mu_{j}, t \geq 0, \quad 1 \leq j \leq N
$$

where $\mu_{j}$ are the relaxation parameters and $c_{v}=2 m \omega_{n} \zeta$ is, as before, the damping coefficient of the viscous model when $\mu_{j} \rightarrow \infty$. The Laplace transform of Eq. (4.57) can be expressed as

$$
G\left(s, \zeta, \mu_{1}, \ldots, \mu_{N}\right)=\frac{2 m \omega_{n} \zeta}{N} \sum_{j=1}^{N} \frac{\mu_{j}}{s+\mu_{j}}
$$




$$
\zeta=0.01
$$
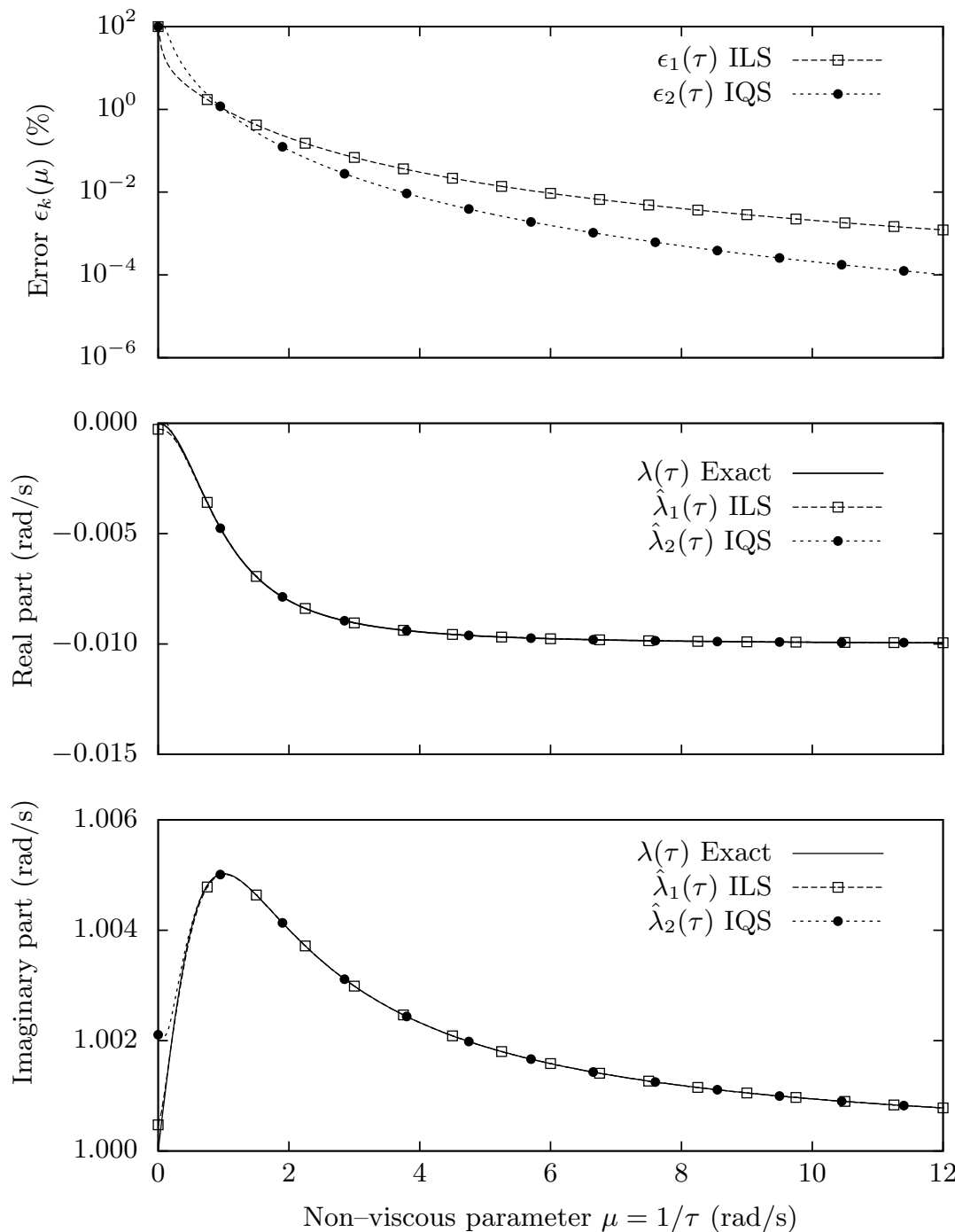

Figure 4.6: Case $\theta=\tau=1 / \mu$. Relative error $\epsilon_{k}(\tau)=\left|\Psi-\psi_{k}\right| /|\Psi|$ of the integrand function (top). Eigenvalues vs. parameter $\mu$ (middle, bottom) for $\zeta=0.01$. Continuous line for exact values and dotted for Improved Solutions; square tick for Linear (ILS), bullet for Quadratic (IQS). 


$$
\zeta=0.05
$$
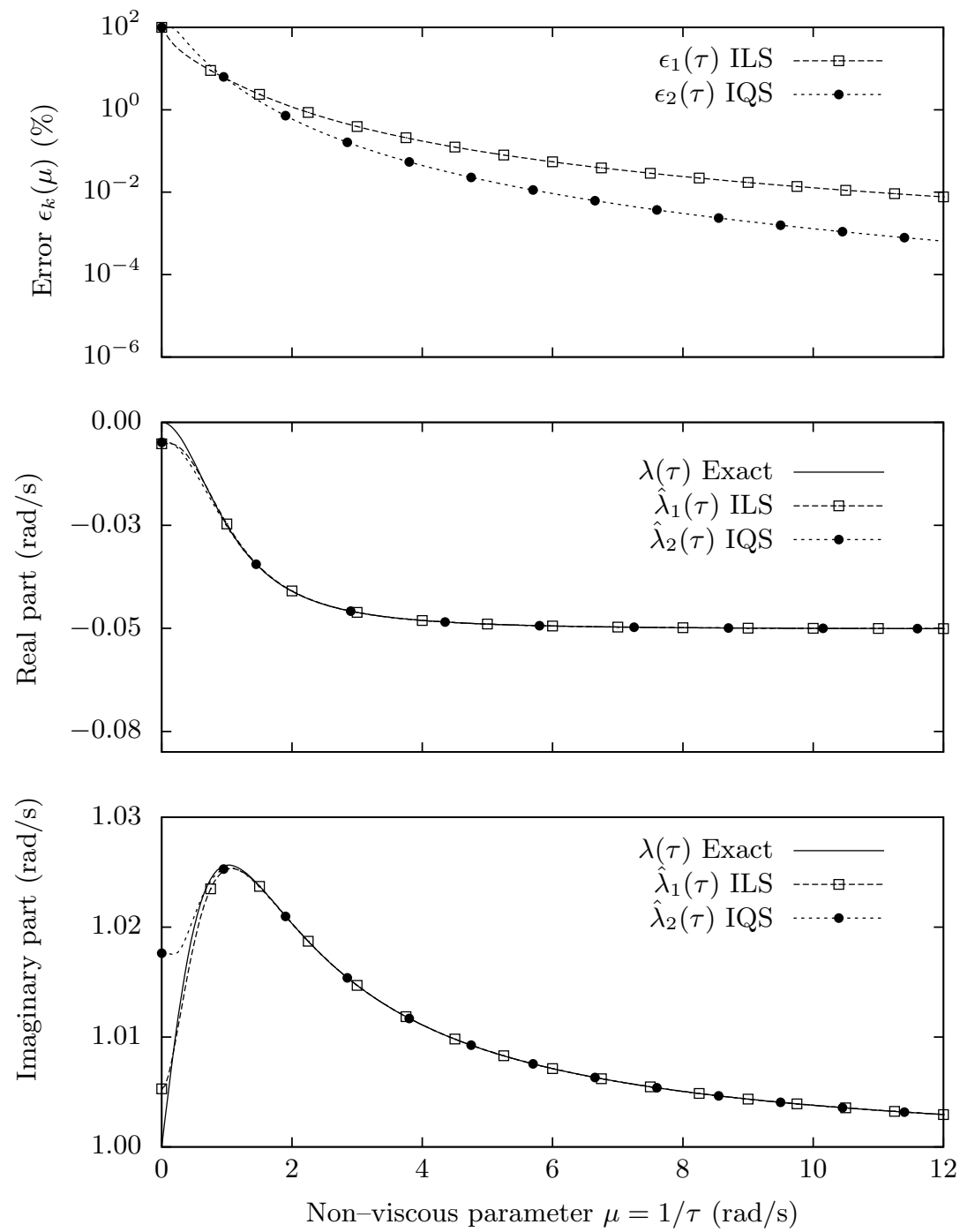

Figure 4.7: Case $\theta=\tau=1 / \mu$. Relative error $\epsilon_{k}(\tau)=\left|\Psi-\psi_{k}\right| /|\Psi|$ of the integrand function (top). Eigenvalues vs. parameter $\mu$ (middle, bottom) for $\zeta=0.05$. Continuous line for exact values and dotted for Improved Solutions; square tick for Linear (ILS), bullet for Quadratic (IQS). 


$$
\zeta=0.10
$$
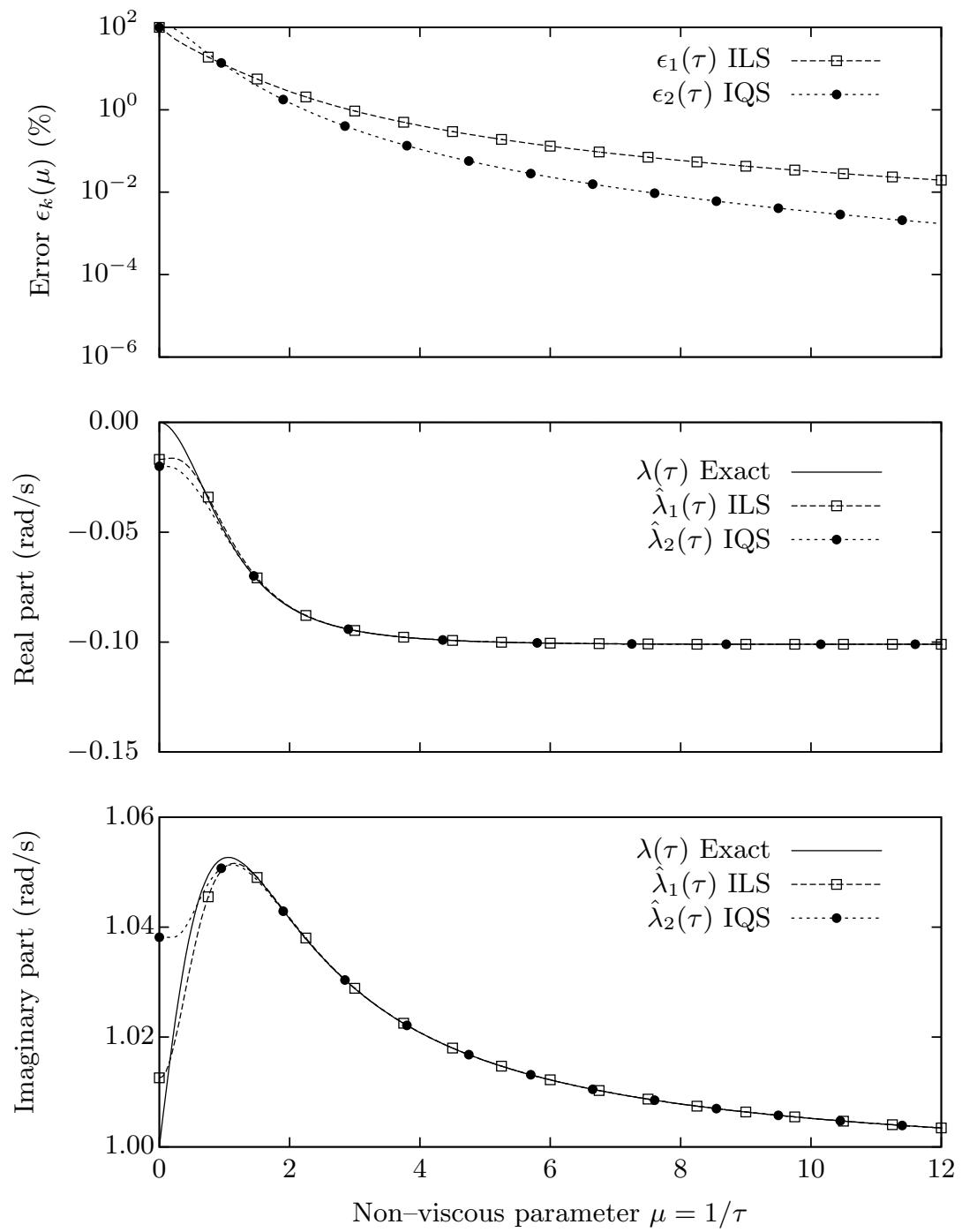

Figure 4.8: Case $\theta=\tau=1 / \mu$. Relative error $\epsilon_{k}(\tau)=\left|\Psi-\psi_{k}\right| /|\Psi|$ of the integrand function (top). Eigenvalues vs. parameter $\mu$ (middle, bottom) for $\zeta=0.10$. Continuous line for exact values and dotted for Improved Solutions; square tick for Linear (ILS), bullet for Quadratic (IQS). 
Directly, the mass-normalized characteristic equation takes the form

$$
\lambda^{2}+2 \lambda \frac{\omega_{n} \zeta}{N} \sum_{j=1}^{N} \frac{\mu_{j}}{\lambda+\mu_{j}}+\omega_{n}^{2}=0
$$

Note that the previous equation can be transformed into a polynomial of order $2+N$. The set formed by the $2+N$ eigenvalues can be separated, on one side, in a subset with the two complex conjugate eigenvalues and, on the other, in another with the $N$ non-viscous eigenvalues that in general are real and negative. The parameters $\mu_{j}$ cannot be used with the proposed method because analytical solutions at the initial point for $N$ kernels are not available. However, if the damping ratio $\zeta$ is used as parameter, the choice of initial value $\zeta_{0}=0$ permits to obtain closed solutions for both the complex eigenvalues and the non-viscous ones.

Complex eigenvalues. The value $\zeta_{0}=0$ produces the eigenvalues of the undamped system in Eq. (4.59), i.e. $\lambda_{0}= \pm i \omega_{n}$. Expanding the solution in its Taylor series around this point, the proposed method allows to obtain $\lambda=\lambda(\zeta)$. The first and second derivatives, $\lambda^{\prime}(0), \lambda^{\prime \prime}(0)$ can be calculated from Eqs. (4.15), (4.17). After some operations

$$
\begin{aligned}
\lambda^{\prime}(0) & =-\frac{\omega_{n}}{N} \sum_{j=1}^{N} \frac{\mu_{j}}{\lambda_{0}+\mu_{j}} \\
\lambda^{\prime \prime}(0) & =\lambda^{\prime}(0)\left[\frac{\lambda^{\prime}(0)}{\lambda_{0}}+\frac{2}{N} \sum_{j=1}^{N} \frac{\omega_{n} \mu_{j}}{\left(\lambda_{0}+\mu_{j}\right)^{2}}\right]
\end{aligned}
$$

The second order Taylor series become function of $\zeta$ : $\lambda_{2}(\zeta)=\lambda_{0}+\lambda^{\prime}(0) \zeta+$ $\lambda^{\prime \prime}(0) \zeta^{2} / 2 \equiv \lambda_{1}(\zeta)+\lambda^{\prime \prime}(0) \zeta^{2} / 2$. As in the previous cases, this approximation can be improved solving the differential Eq. (4.23), for which

$$
\psi_{k}(\zeta)=\left[\zeta+\lambda_{k}(\zeta) \frac{1-\frac{\zeta \omega_{n}}{N} \sum_{j=1}^{N} \frac{\mu_{j}}{\left(\lambda_{k}(\zeta)+\mu_{j}\right)^{2}}}{\frac{\omega_{n}}{N} \sum_{j=1}^{N} \frac{\mu_{j}}{\left(\lambda_{k}(\zeta)+\mu_{j}\right)}}\right]^{-1}
$$

The method is implemented for a numerical example with $N=5$ kernels. The relaxation parameters are taken as $\mu_{j}=\{2,4,6,10,15\} \mathrm{rad} / \mathrm{s}$. Three different natural frequencies $\omega_{n}=\{0.2,2,20\} \mathrm{rad} / \mathrm{s}$ have been considered, to validate the results when the system is near-viscous $\mu_{j}>>\omega_{n}$ or, otherwise non-viscous $\mu_{j} \approx \omega_{n}$. 


$$
\omega_{n}=0.20 \mathrm{rad} / \mathrm{s}
$$
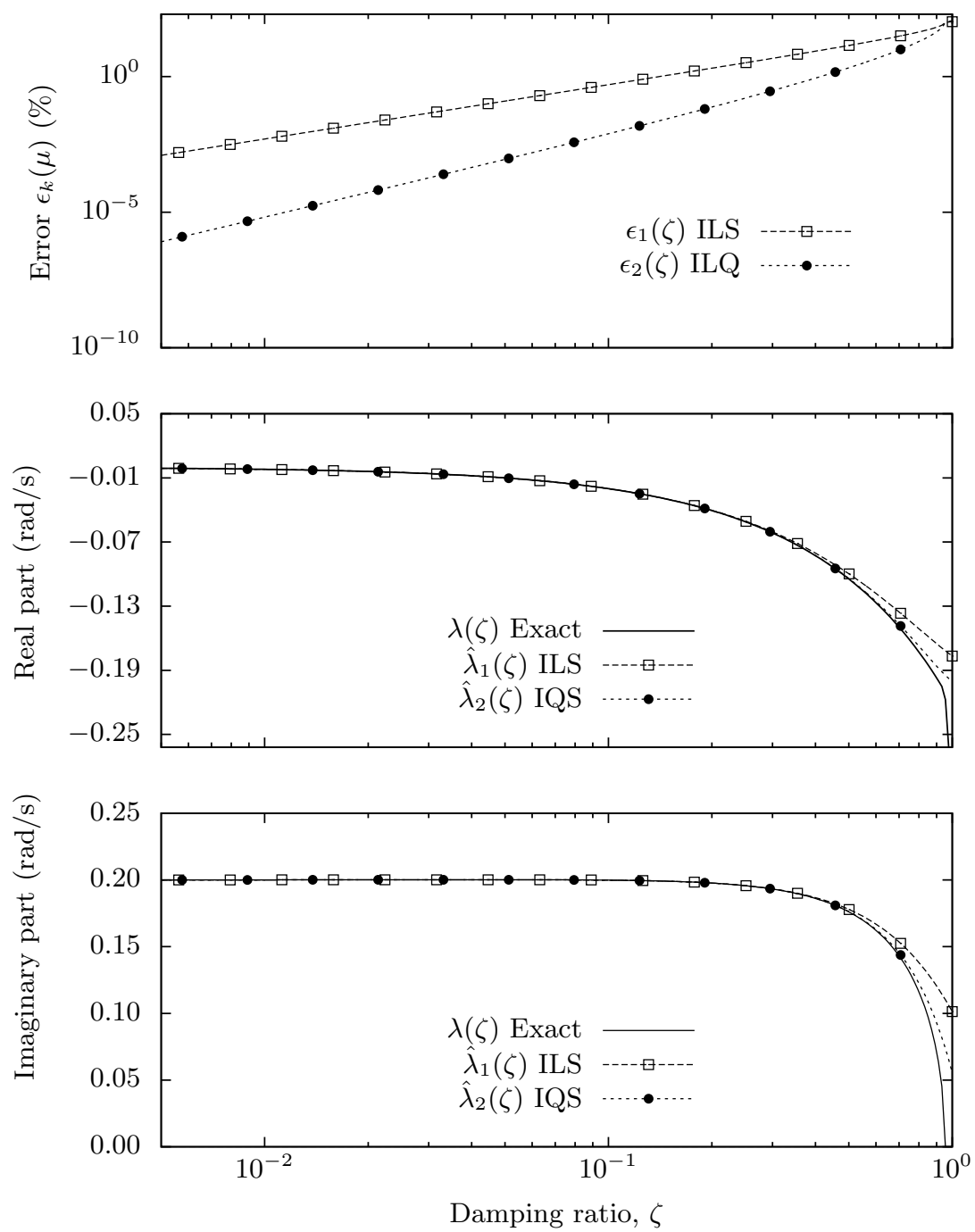

Figure 4.9: Case $\theta=\zeta$ for Biot's multiexponential model $\mu_{j}=\{2,4,6,10,15\} \mathrm{rad} / \mathrm{s}$. Relative error $\epsilon_{k}(\zeta)=\left|\Psi-\psi_{k}\right| /|\Psi|$ of the integrand function (top). Eigenvalues vs. parameter $\zeta$ (middle, bottom) for $\omega_{n}=0.20 \mathrm{rad} / \mathrm{s}$. Continuous line for exact values and dotted for Improved Solutions; square tick for Linear (ILS), bullet for Quadratic (IQS). 


$$
\omega_{n}=2.00 \mathrm{rad} / \mathrm{s}
$$
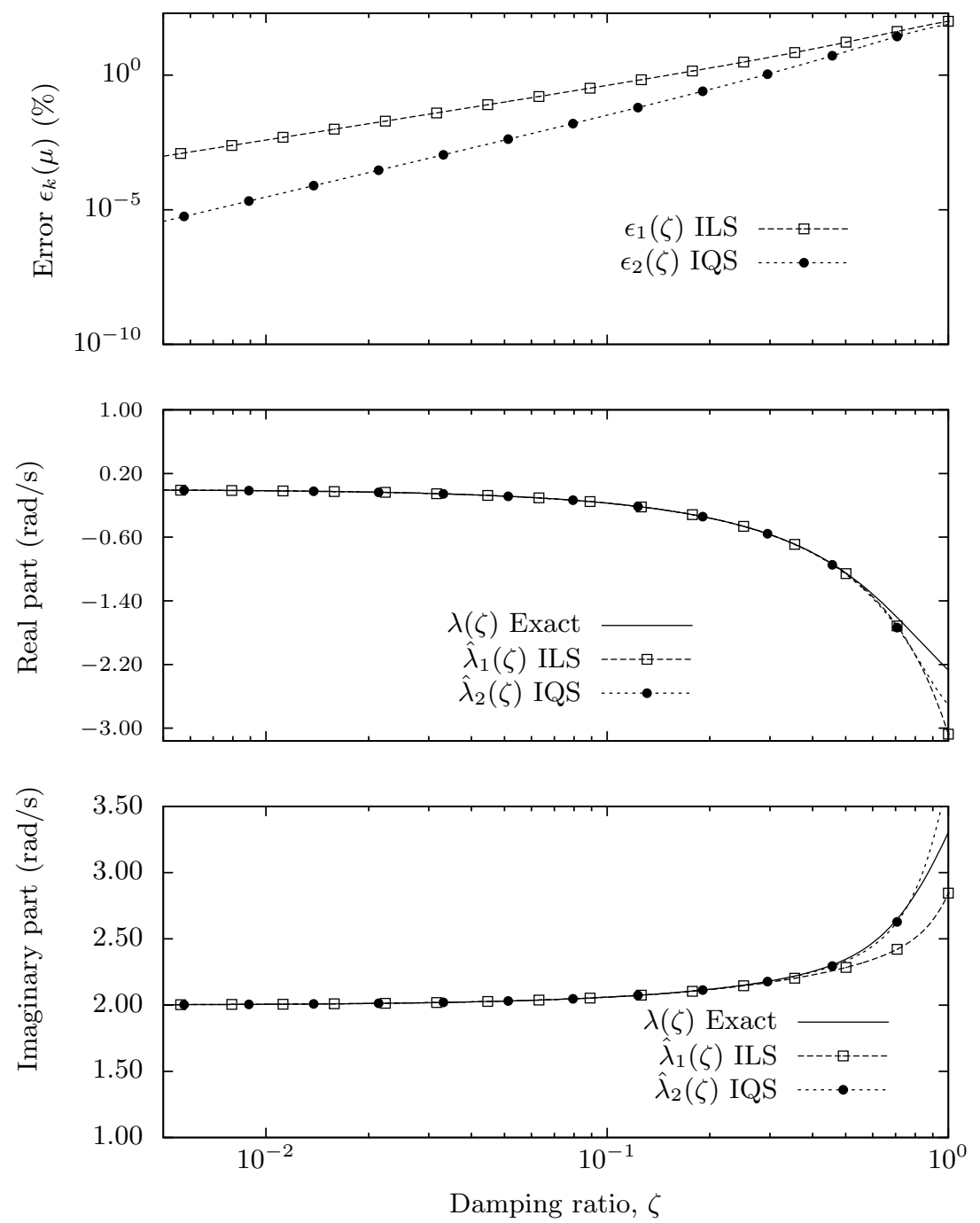

Figure 4.10: Case $\theta=\zeta$ for Biot's multiexponential model $\mu_{j}=\{2,4,6,10,15\} \mathrm{rad} / \mathrm{s}$. Relative error $\epsilon_{k}(\zeta)=\left|\Psi-\psi_{k}\right| /|\Psi|$ of the integrand function (top). Eigenvalues vs. parameter $\zeta$ (middle, bottom) for $\omega_{n}=2.00 \mathrm{rad} / \mathrm{s}$. Continuous line for exact values and dotted for Improved Solutions; square tick for Linear (ILS), bullet for Quadratic (IQS). 


$$
\omega_{n}=20.0 \mathrm{rad} / \mathrm{s}
$$
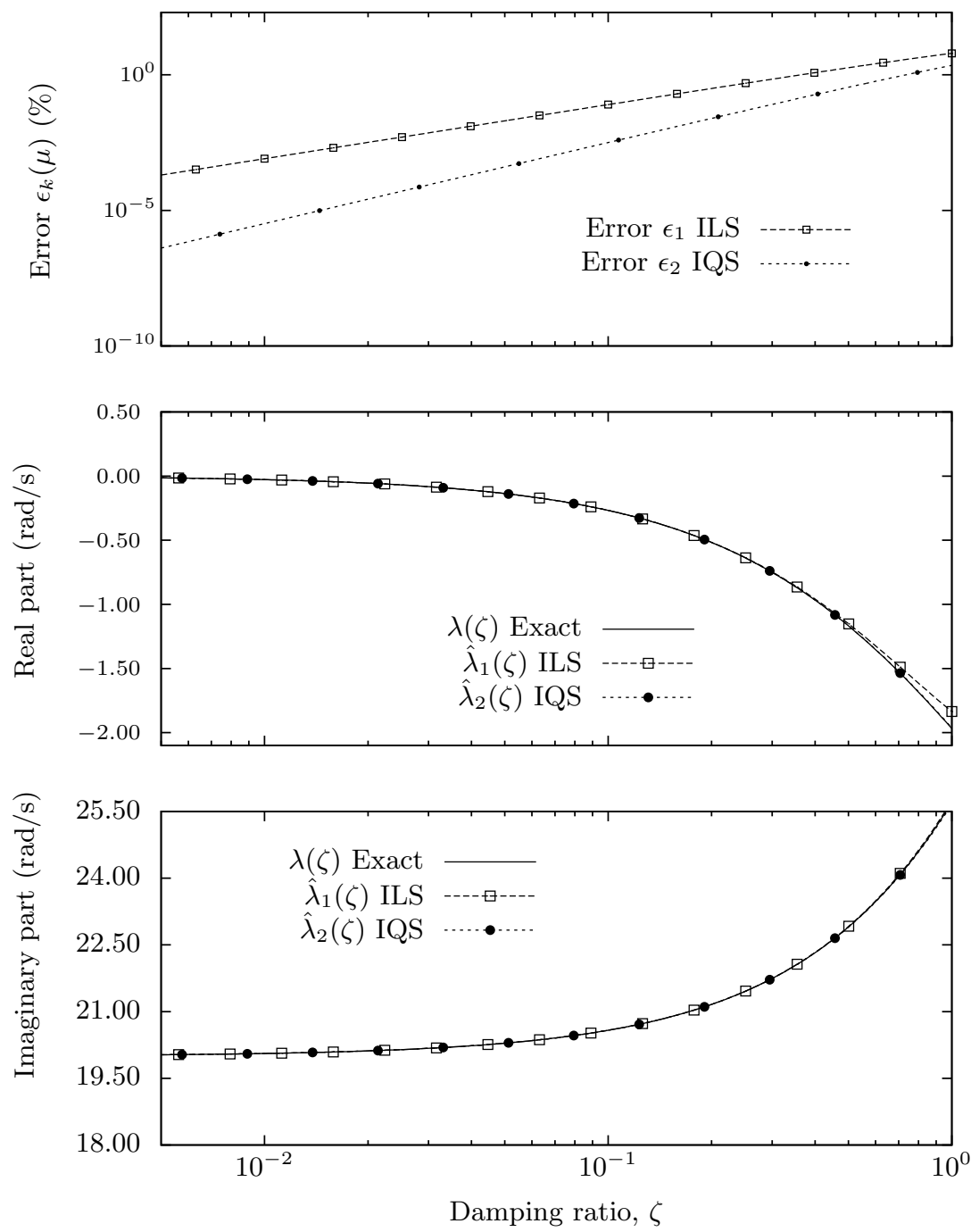

Figure 4.11: Case $\theta=\zeta$ for Biot's multiexponential model $\mu_{j}=\{2,4,6,10,15\} \mathrm{rad} / \mathrm{s}$. Relative error $\epsilon_{k}(\zeta)=\left|\Psi-\psi_{k}\right| /|\Psi|$ of the integrand function (top). Eigenvalues vs. parameter $\zeta$ (middle, bottom) for $\omega_{n}=20.0 \mathrm{rad} / \mathrm{s}$. Continuous line for exact values and dotted for Improved Solutions; square tick for Linear (ILS), bullet for Quadratic (IQS). 
Figs. 4.9-4.11 show the errors of the integrand function and the approximated eigenvalues $\hat{\lambda}_{1}(\zeta), \hat{\lambda}_{2}(\zeta)$ as before. The damping ratio $\zeta$ has again been represented log-scaled. As in the previous case $\epsilon_{2}(\zeta)<\epsilon_{1}(\zeta)$ (several orders of magnitude) $\forall \zeta \in[0,1]$, obtaining better approximations for IQS than for ILS. For both viscous and non-viscous system the agreement is very good, except for $\zeta \approx 1$ and $\omega_{n}=0.20$ $\mathrm{rad} / \mathrm{s}$ (viscous behavior), where the slope of the imaginary part becomes almost vertical.

Real eigenvalues It is expected that the real eigenvalues of Eq. (4.58) will be relatively close to the parameters $\mu_{j}$ in lightly damped systems, as described by Adhikari and Pascual [32]. Naming $\lambda=\lambda(\zeta)$ to the non-viscous eigenvalue associated with a particular parameter $\mu_{r}$, then $\lambda_{0}=\lim _{\zeta \rightarrow 0} \lambda(\zeta)=-\mu_{r}$. Indeed, multiplying Eq. (4.59) by $\lambda+\mu_{r}$ results in

$$
\left[\lambda(\zeta)+\mu_{r}\right]\left[\lambda^{2}(\zeta)+\omega_{n}^{2}\right]+2 \lambda(\zeta) \frac{\omega_{n} \zeta}{N} \mu_{r}++2 \lambda(\zeta) \frac{\omega_{n} \zeta}{N} \sum_{\substack{j=1 \\ j \neq r}}^{N} \frac{\lambda(\zeta)+\mu_{r}}{\lambda(\zeta)+\mu_{j}} \mu_{j}=0
$$

Taking limits $\zeta \rightarrow 0$ in the previous expression

$$
\left(\lambda_{0}+\mu_{r}\right)\left(\lambda_{0}^{2}+\omega_{n}^{2}\right)=0
$$

Since $\lambda_{0} \in \mathbb{R}$ for the non-viscous eigenvalues, $\lambda_{0}^{2}+\omega_{n}^{2}>0$ and consequently $\lambda_{0}+$ $\mu_{r}=0$. Therefore, the non-viscous eigenvalue associated with $\mu_{j}$ can be expanded around the point $\lambda_{0}=\lambda(0)=-\mu_{j}$. Without loss of generality, it can always be assumed that $\lambda(\zeta)$ is associated with the first parameter $\mu_{1}$. Thus, for any value $\zeta>0$, the Taylor series expansion is now $\lambda_{2}(\zeta)=-\mu_{1}+\lambda^{\prime}(0) \zeta+\lambda^{\prime \prime}(0) \zeta^{2} / 2$. Since the viscoelastic function and its derivatives are not defined in $s=-\mu_{j}$ (see Eq. (4.58)), the derivatives from Eqs. (4.15), (4.17) can not be calculated. To avoid this problem, Eq. (4.62) with $r=1$ will be used. Thus, $\lambda^{\prime}(0), \lambda^{\prime \prime}(0)$ can be calculated taking derivatives respect to $\zeta$ in the expression

$$
\left[\lambda(\zeta)+\mu_{1}\right]\left[\lambda^{2}(\zeta)+\omega_{n}^{2}\right]++2 \lambda(\zeta) \frac{\omega_{n} \zeta}{N}\left(\mu_{1}+\sum_{j=2}^{N} \frac{\lambda(\zeta)+\mu_{1}}{\lambda(\zeta)+\mu_{j}} \mu_{j}\right)=0
$$

Evaluating Eq. (4.64) at $\zeta=0$ and after some operations, the following results are obtained

$$
\begin{aligned}
\lambda^{\prime}(0) & =\frac{2 \omega_{n} \mu_{1}^{2}}{\omega_{n}^{2}+\mu_{1}^{2}} \\
\lambda^{\prime \prime}(0) & =\frac{8 \omega_{n}^{2} \mu_{1}^{3}}{\left(\omega_{n}^{2}+\mu_{1}^{2}\right)^{2}}\left[\frac{\mu_{1}^{2}-\omega_{n}^{2}}{\omega_{n}^{2}+\mu_{1}^{2}}+\sum_{j=2}^{N} \frac{\mu_{j}}{\mu_{j}-\mu_{1}}\right]
\end{aligned}
$$

The computation of the improved solutions from the differential Eq. (4.20) requires that $G(s)$ is well defined at the initial point. Since $\lambda_{0}=-\mu_{j}$ is a pole of $G(s)$ 
and of $\partial G(s) / \partial s$, the Taylor series up to the first and second order will be used to estimate the eigenvalues.

Fig. 4.12 shows results for a numerical example with $\omega_{n}=3 \mathrm{rad} / \mathrm{s}$ and Biot's model with $N=3$ kernels and $\mu_{j}=\{1,5,10\} \mathrm{rad} / \mathrm{s}$. As expected, the eigenvalues show very good accuracy for damping range $0 \leq \zeta \leq 0.20$. Better results could be achieved through expansion of the Taylor series up to higher orders. For that, successive derivatives $\lambda^{\prime \prime \prime}(0), \lambda^{I V}(0), \ldots$ from Eq. (4.64) can be extracted and new terms added.

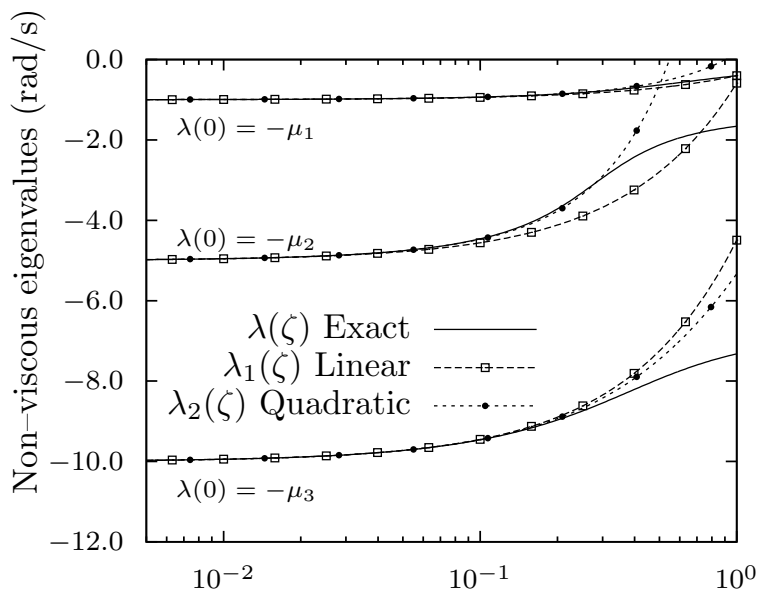

Damping ratio, $\zeta$

Figure 4.12: Exact solution and Taylor series approximation (quadratic, linear) of the non-viscous eigenvalues vs. damping ratio $\zeta$. Biot's model with $N=3$ kernels and non-viscous parameters $\mu_{j}=\{1,5,10\} \mathrm{rad} / \mathrm{s}$.

\subsection{Conclusions}

A novel numerical method to compute the eigenvalues of single degree-of-freedom linear viscoelastic systems is developed. The damping is introduced via a convolution integral with kernel functions that ensure that the dissipative forces depend on the history of the degree-of-freedom velocities. Each damping model is controlled by a kernel involving a set of parameters; the eigenvalues can be considered single-variable functions of some of these damping parameters. The application of the method requires to find only a particular value of the damping parameter and its corresponding eigenvalue. Under certain conditions, the eigenvalue can 
be expanded in its Taylor series and explicit expressions of the first and second derivatives are calculated.

The main contribution of this paper is the development of a numerical approach that improves the solution given by the Taylor series expansion. For that, it is shown that the characteristic equation always can be transformed into an ordinary differential equation with separated variables, so that the eigenvalue can be estimated by direct numerical integration. In addition, the order error of the proposed method is analyzed; while the $k$ th order Taylor series expansion presents $\mathcal{O}\left(h^{k+1}\right)$, it is demonstrated that the current method presents $\mathcal{O}\left(h^{k+2}\right)$.

To illustrate the results, the method is applied to two commonly used damping models: viscous damping and non-viscous damping with exponential kernels. For the former, the method obtains approximated eigenvalues as analytical solutions of the proposed differential equation. For the latter, several forms of the damping parameters are presented, finding again analytical solutions for the Taylor expansions for all forms. The numerical examples show that the lower the damping level, the better the accuracy of the proposed solutions is. The best results are always obtained when the parameter value is close to the considered initial point. Further research could be oriented in two directions: (a) the implementation of the proposed method with other damping functions, for instance those based on the fractional derivative; (b) the extension for multiple degrees-of-freedom along with a multivariable parameter. Both directions are currently being developed.

\section{Appendix 4.A Proof of the Lemma 1}

The function $\Psi(s, \theta)$ is well defined, provided that the denominator

$$
\left|\Gamma(s, \theta)+s\left(2+\frac{\partial \Gamma}{\partial s}\right)\right|>0 \quad \forall s, \theta \in B \times I
$$

Hence, let $L_{0}$ be a positive real number, such that

$$
L_{0}^{-1}=\min \left\{\left|\Gamma(s, \theta)+s\left(2+\frac{\partial \Gamma}{\partial s}\right)\right|: s, \theta \in B \times I\right\}
$$

In addition, according to the hypothesis $\mathrm{H} 2$, the function $G(s, \theta)=m \Gamma(s, \theta)$ and its derivatives respect to $\theta$ are bounded in the set $B \times I$. Thus, a positive real number $r_{0}$ exists, such that

$$
\left|\frac{\partial \Gamma}{\partial \theta}\right| \leq r_{0} \quad, \forall(s, \theta) \in B \times I
$$


Therefore, taking into account Eq. (4.67), (4.68) the function $\Psi(s, \theta)$ can be bounded by the positive real number $p=r_{0} L_{0}$

$$
\begin{aligned}
|\Psi(s, \theta)| & =\left|\frac{\Gamma_{\theta}^{\prime}(s, \theta)}{\Gamma(s, \theta)+\lambda\left[2+\Gamma_{s}^{\prime}(s, \theta)\right]}\right| \\
& \leq r_{0} L_{0}=p \quad \forall s, \theta \in B \times I
\end{aligned}
$$

\section{Appendix 4.B Proof of the Lemma 2}

The function $\lambda(\theta)$ is solution of the characteristic equation, therefore the following expression is verified

$$
\sigma^{2}(\theta)+\sigma(\theta) \frac{G(\lambda(\theta), \theta)}{m \omega_{n}}+1=0
$$

where the function $\sigma(\theta)=\lambda(\theta) / \omega_{n}$. By hypothesis, the function $G(s, \theta)$ is bounded in the set $B \times I$. Hence, there exists a real positive number $\alpha>0$ such that $|G(s, \theta)| \leq 2 \alpha m \omega_{n}, \forall s, \theta \in B \times I$. Reordering Eq. (4.70) and taking absolute values

$$
\begin{aligned}
|\sigma(\theta)|^{2} & =\left|\sigma(\theta) \frac{G(\lambda(\theta), \theta)}{m \omega_{n}}+1\right| \\
& \leq|\sigma(\theta)|\left|\frac{G(\lambda(\theta), \theta)}{m \omega_{n}}\right|+1 \\
& \leq 2 \alpha|\sigma(\theta)|+1
\end{aligned}
$$

As a consequence, the function $\sigma(\theta)$ verifies the inequality

$$
|\sigma(\theta)|^{2}-2 \alpha|\sigma(\theta)|-1 \leq 0
$$

The roots of the second order polynomial $x^{2}-2 \alpha x-1=0$ are the real numbers $\alpha \pm \sqrt{1+\alpha^{2}}$. Therefore, the function $|\sigma(\theta)|$ is bounded by the interval

$$
0 \leq|\sigma(\theta)| \leq \alpha+\sqrt{1+\alpha^{2}}
$$

because $\alpha-\sqrt{1+\alpha^{2}}<0$. Finally, the expression of the eigenvalue bound leads to

$$
|\lambda(\theta)| \leq \omega_{n}\left(\alpha+\sqrt{1+\alpha^{2}}\right)=\rho_{0} \quad \forall \theta \in I
$$

In order to bound the derivatives $\left|\lambda^{k}(\theta)\right|, k \geq 1$, Eq. (4.20) relates the first with functions $\lambda(\theta), \Psi(s, \theta)$, whose bounds have already been calculated by Eqs. (4.69), (4.74). Thus,

$$
\left|\lambda^{\prime}(\theta)\right| \leq|\lambda(\theta)||\Psi(\lambda(\theta), \theta)| \leq \rho_{0} p=\rho_{1} \quad \forall \theta \in I
$$


Higher derivatives of $\Psi(\lambda(\theta), \theta)$ respect the parameter $\theta$ will be expressions function of the successive derivatives of the viscoelastic function $\Gamma(\lambda(\theta), \theta)$ that, from the hypothesis $\mathrm{H} 2$, are bounded in the interval $I$. Consequently, once calculated $\rho_{0}$ and $\rho_{1}$, the numbers $\rho_{k}$ for $k=2,3, \ldots$ can be obtained by induction. 


\section{Bibliography}

[7] M.A. Biot. "Variational Principles in Irreversible Thermodynamics with Application to Viscoelasticity." In: Physical Review 97.6 (1955), 1463-1469 (cit. on pp. 3, 20, 24, 37, 58, 82, 93, 192).

[14] D.F. Golla and P.C. Hughes. "Dynamics of Viscoelastic Structures - A Time-domain, Finite-element Formulation." In: Journal of Applied MechanicsTransactions of the ASME 52.4 (1985), 897-906 (cit. on pp. 3, 20, 22, 24, $58,60,82,84,118,160,162,164,192)$.

[15] R.L. Bagley and P.J. Torvik. "A Theoretical Basis for the Application of Fractional Calculus to Viscoelasticity." In: Journal of Rheology 27.3 (1983), 201-210 (cit. on pp. 4, 20, 82, 192, 196).

[16] R.L. Bagley and P.J. Torvik. "Fractional Calculus - A Different Approach to the Analysis of Viscoelastically Damped Structures." In: AIAA Journal 21.5 (1983), 741-748 (cit. on pp. 4, 20, 24, 82, 192, 193, 196).

[28] S. Adhikari. "Dynamics of Non-viscously Damped Linear Systems." In: Journal of Engineering Mechanics 128.3 (2002), 328-339 (cit. on pp. 4-6, $22,35,36,58,84,94,121,125,129,131,160-162,165,168,170,171,203)$.

[29] S. Adhikari. "Classical normal modes in non-viscously damped linear systems." In: AIAA Journal 39.5 (2001), pp. 978-980 (cit. on pp. 5, 35, 44, $63,84,121)$.

[31] Sondipon Adhikari and Blanca Pascual. "Eigenvalues of linear viscoelastic systems." In: Journal of Sound and Vibration 325.4-5 (2009), 1000-1011 (cit. on pp. 5, 21, 35, 41, 42, 69, 70, 76, 83, 119, 120, 141, 151, 152, 193).

[32] Sondipon Adhikari and Blanca Pascual. "Iterative Methods for Eigenvalues of Viscoelastic Systems." In: Journal of Vibration and Acoustics 133.2 (2011), pp. 021002.1-021002.7 (cit. on pp. 5, 21, 35, 42, 59, 83, 108, 119, 171, 193).

[40] D.J. McTavish and P.C. Hughes. "Modeling of Linear Viscoelastic Space Structures." In: Journal of Vibration and Acoustics-Transactions of the ASME 115.1 (1993), 103-110 (cit. on pp. 20, 24, 58, 82, 118, 160, 164). 
[41] GA Lesieutre and DL Mingori. "Finite-Element Modeling of Frequencydependent Material Damping Using Augmenting Thermodynamic Fields." In: Journal of Guidance Control and Dynamics 13.6 (1990), 1040-1050 (cit. on pp. $20,24,58,82,119)$.

[43] S. Adhikari and J. Woodhouse. "Identification Of Damping: PART 2, NonViscous Damping." In: Journal of Sound and Vibration 243.1 (2001), 63-88 (cit. on pp. 20, 24, 82).

[46] Wei H. Yang. "A method for eigenvalues of sparse $\lambda$-matrices." In: International Journal for Numerical Methods in Engineering 19.6 (1983), pp. 943948 (cit. on pp. 21, 83, 118, 193).

[47] KV Singh and YM Ram. "Transcendental eigenvalue problem and its applications." In: AIAA Journal 40.7 (2002), 1402-1407 (cit. on pp. 21, 83, 118).

[48] FW Williams and D Kennedy. "Reliable Use of Determinants to Solve Non-linear Structural Eigenvalue Problems Efficiently." In: International Journal for Numerical Methods in Engineering 26.8 (1988), 1825-1841 (cit. on pp. 21, 83, 118).

[49] E. M. Daya and M. Potier-Ferry. "A numerical method for nonlinear eigenvalue problems application to vibrations of viscoelastic structures." In: Computers 85 Structures 79.5 (2001), pp. 533 -541 (cit. on pp. 21, 83, 119).

[51] H. Voss. "An Arnoldi Method for Nonlinear Eigenvalue Problems." In: BIT Numerical Mathematics 44 (2 2004), pp. 387-401 (cit. on pp. 21, 83, 118).

[52] H. Voss. "A Jacobi-Davidson method for nonlinear and nonsymmetric eigenproblems." In: Computers \& Structures 85.17-18 (2007), 1284-1292 (cit. on pp. 21, 83, 118).

[54] A Muravyov and SG Hutton. "Closed-form solutions and the eigenvalue problem for vibration of discrete viscoelastic systems." In: Journal of Applied Mechanics-Transactions of the ASME 64.3 (1997), 684-691 (cit. on pp. 21, 58, 83, 119, 160, 164, 193).

[55] A. Muravyov. "Forced vibration responses of viscoelastic structure." In: Journal of Sound and Vibration 218.5 (1998), 892-907 (cit. on pp. 21, 38, $83,119,160)$.

[56] S. Menon and J. Tang. "A state-space approach for the dynamic analysis of viscoelastic systems." In: Computers \& Structures 82.15-16 (2004), pp. 1123 -1130 (cit. on pp. 21, 83, 119, 160, 164, 193).

[59] S. Adhikari. "Derivative of Eigensolutions of Nonviscously Damped Linear Systems." In: AIAA Journal 40.10 (2002), 2061-2069 (cit. on pp. 21, 83, $119,126,132)$. 
[60] F. Cortés and Maria Jesús Elejabarrieta. "Computational methods for complex eigenproblems in finite element analysis of structural systems with viscoelastic damping treatments." In: Computer Methods Appl. Mech. Engineering 195.44-47 (2006), 6448-6462 (cit. on pp. 21, 83, 119).

[61] F. Cortés and Maria Jesús Elejabarrieta. "An approximate numerical method for the complex eigenproblem in systems characterised by a structural damping matrix." In: Journal of Sound and Vibration 296.1-2 (2006), 166182 (cit. on pp. 21, 83, 119).

[64] P Muller. "Are the eigensolutions of a l-d.o.f. system with viscoelastic damping oscillatory or not?" In: Journal of Sound and Vibration 285.12 (2005), 501-509 (cit. on pp. 22, 38, 58, 84, 121, 162).

[71] S. Adhikari and J. Woodhouse. "Quantification of non-viscous damping in discrete linear systems." In: Journal of Sound and Vibration 260.3 (2003), pp. 499-518 (cit. on pp. 33, 88, 94, 125, 197, 203).

[74] S Adhikari. "Qualitative dynamic characteristics of a non-viscously damped oscillator." In: Proceedings of the Royal Society A-Mathematical Physical and Engineering Sciences 461.2059 (2005), 2269-2288 (cit. on pp. 38, 58, $84,121)$.

[78] S. Adhikari and J. Woodhouse. "Identification Of Damping: PART 1, Viscous Damping." In: Journal of Sound and Vibration 243.1 (2001), 43-61 (cit. on p. 82).

[79] RL Fox and MP Kapoor. "Rates of Change Eigenvalues and Eigenvectors." In: AIAA Journal 6.12 (1968), 2426-\& (cit. on pp. 83, 119).

[80] RB Nelson. "Simplified Calculation of Eigenvector Derivatives." In: AIAA Journal 14.9 (1976), 1201-1205 (cit. on pp. 83, 119).

[81] BP Wang. "Improved Approximate Methods for Computing Eigenvector Derivatives in Structural Dynamics." In: AIAA Journal 29.6 (1991), 1018 1020 (cit. on pp. 83, 119).

[82] OQ Zhang and A Zerva. "Iterative method for calculating derivatives of eigenvectors." In: AIAA Journal 34.5 (1996), 1088-1090 (cit. on pp. 83, 119).

[83] DV Murthy and RT Haftka. "Derivatives of Eigenvalues and Eigenvectors of a General Complex Matrix." In: International Journal for Numerical Methods in Engineering 26.2 (1988), 293-311 (cit. on pp. 83, 119).

[84] DV Murthy and RT Haftka. "Approximations to Eigenvalues of Modified General Matrices." In: Computers $\&$ Structures 29.5 (1988), 903-917 (cit. on pp. 83,119$)$. 
[85] S. Adhikari. "Rates of change of eigenvalues and eigenvectors in damped dynamic system." In: AIAA Journal 37.11 (1999), 1452-1458 (cit. on pp. 83, 119). 


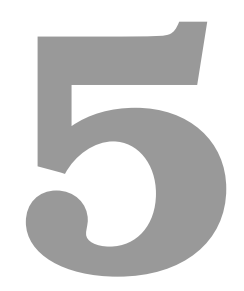

\section{Multiparametric Computation of Eigenvalues for Linear Viscoelastic Structures}

Computers \& Structures, 117 (2013), 67-81

\subsection{Introduction}

The mechanisms of energy dissipation in mechanical systems are complex in nature, due to the difficulty to translate the interactions between particles from the microscopic to the macroscopic level. The most used models are based on linear relationships between the dissipative forces and the degree-of-freedom (dof) velocities. Among these models, the viscous is still the simplest and traditionally the most popular for practical applications of aeronautical, industrial and civil engineering. When traditional materials are involved, the stress-strain constitutive relationships are frequency-dependent; it is then necessary to use the so called nonviscous or viscoelastic models and the related dissipative forces are proportional to the history of the dof velocities via convolution integrals involving kernel functions. 
The response of any multiple dof system satisfies the dynamic equilibrium given by the following system of integro-differential equations

$$
\mathbf{M} \ddot{\mathbf{u}}+\int_{-\infty}^{t} \mathcal{G}(t-\tau) \dot{\mathbf{u}} \mathrm{d} \tau+\mathbf{K u}=\mathbf{F}(t)
$$

where $\mathbf{M}, \mathbf{K} \in \mathbb{R}^{N \times N}$ are the assembled mass and stiffness matrices. It is commonly assumed that $\mathbf{M}$ is positive definite and $\mathbf{K}$ is positive semidefinite. The function $\mathcal{G}(t) \in \mathbb{R}^{N \times N}$ is the viscoelastic damping matrix that must satisfy the necessary conditions given by Golla and Hughes [14] to induce a dissipative motion in the system. The denominated viscous damping is a particular case for which $\mathcal{G}(t) \equiv \mathbf{C} \delta(t)$, where $\mathbf{C}$ is the viscous damping matrix and $\delta(t)$ the Dirac's function. The solution of the nonlinear eigenvalue problem associated to Eq. (5.1) has a special importance for the analysis of viscoelastic structures. This importance has motivated the development of new numerical techniques to extract complex eigenvalues. The essential of the proposed method is to consider those eigenvalues as functions of certain multi-dimensional vector composed of damping parameters. In the development of the methodology, the knowledge of the eigenvalue derivatives is a key point.

In general, universal techniques for the resolution of nonlinear eigenvalue problems are not available. Current methods are usually oriented to find solutions close to a given initial value. Yang [46], Singh and Ram [47] proposed methods based on linearization via Taylor series expansion of a transcendental matrix around a point. The convergence is achieved with a Newton's iterative scheme and requires a good initial approximation. Voss $[51,52]$ proposed two numerical approaches based on Arnoldi's shift-and-invert and on Jacobi-Davidson methods, respectively. In both of them, an iterative process is used to compute a few eigenvalues close to a given value. Williams and Kennedy [48], obtained solutions proposing polynomial interpolation of the determinant, with the advantage of not requiring derivatives.

The particular form of the nonlinear eigenvalue problem of a viscoelastic structure allows for the use of specific methods as described in this paragraph. The free motion equation can be deduced imposing $\mathbf{F}(t) \equiv \mathbf{0}$ in Eq. (5.1). Trying solutions of the form $\mathbf{u}(t)=\mathbf{u}_{0} e^{s t}$, this equation reduces to the following nonlinear eigenvalue problem

$$
\left[s^{2} \mathbf{M}+s \mathbf{G}(s)+\mathbf{K}\right] \mathbf{u}_{0}=\mathbf{0}
$$

where $\boldsymbol{G}(s)$ is the Laplace Transform of $\mathcal{G}(t)$. Although direct integration methods in time domain are available in the literature [86, 87], state-space approach based methods are widespread when $\boldsymbol{G}(s)$ adopts a rational form. In such cases, the state-space approach transforms the nonlinear problem into a linear one through the introduction of new internal variables. Relevant state-space approaches are the GHM model proposed by Golla, Hughes and McTavish [14, 40] and the Anelastic 
Displacement Field of Lesieutre and Mingori [41]. Other state-space methods are those of Muravyov [54, 55] and Menon [56]. The augmentation of the linear problem depends on the viscoelastic nature of the function $\mathbf{G}(s)$; when the original size is relatively large the problem can become computationally inefficient. Moreover, the introduction of new variables reduces the physical intuition of the problem. Duigou, Daya and Potier-Ferry. [49, 50] developed two methods to compute the eigensolutions of vibrating viscoelastic structures, using perturbation techniques and high order iterative algorithms. In the context of proportional damping systems, Adhikari and Pascual [31, 32] developed iterative methods based on the Taylor series expansion of the viscoelastic function.

Possibly, Rayleigh [88] was the first author to study the natural frequency sensitivity of a dynamic system, sensitivity that arises due to small variations of the model design parameters. Since then, many authors have studied the eigensolutions (eigenvalues and eigenvectors) as functions of the dynamic matrices parameters. Fox and Kapoor [79] published a relevant work involving analytic expressions of the derivatives, but with the necessity of the full eigensolution set. Nelson [80] improved [79] computing the derivatives with the help of only the eigensolutions of interest. Wang [81] and later Zhang and Zerva [82] proposed methods to calculate the derivatives of truncated systems. Murthy and Haftka [83] published a method survey for derivative calculations applied to general problems with non-Hermitian matrices. Murthy [84] studied the computational efficiency of different approximations based on eigenvalue derivatives, generalized Rayleigh quotient and the trace theorem. The generalization of the eigensolution derivatives to non-viscous damped systems was performed by Adhikari [58, 59, 85]. Cortés and Elejabarrieta $[60,61]$ proposed an iterative numerical method to compute the eigensolutions again based on the derivatives, including highly damped systems. Lázaro et al. [33] have recently developed a recursive scheme for proportionally systems, which does not need derivatives; furthemore, this approach presents global convergence for any chosen initial point.

The present paper aims to develop a new numerical approach to solve the nonlinear eigenvalue problem associated with linear viscoelastic structures. It is assumed that the damping model depends on a certain array of parameters, so that the eigenvalues may be considered multivariable functions of them. The assessment of the eigenvalue derivatives with respect to the parameter array allows to construct the Taylor linear approximation. The proposed method transforms the characteristic equation into an ordinary differential equation and provides an approximated solution. It is rigorously demonstrated that this solution improves the Taylor linear solution. The numerical scheme is developed for proportional as well as non-proportional damping systems. For the latter, two alternatives based on the procedure to compute the eigenvalue derivative are proposed: 1 ) the first 
alternative uses the determinant of the dynamic stiffness matrix (DETD) and 2) the second alternative, computationally more efficient, uses an approximation of the associated eigenvector (EIGD) .

To illustrate the theoretical results three numerical examples are studied: Examples 1 and 2 analyze discrete systems with proportional and non-proportional damping, respectively. For both examples, it is concluded that the use of eigenvalues associated with values relatively close to a chosen initial point shows a better approximation, as demonstrated by theoretical results. Example 3 validates the proposed method for a continuous beam with free damping layers with a discrete viscoelastic damper. The obtained results for each example are compared with those of the numerical approach of Adhikari and Pascual [31], valid for proportional damping. In general, the stronger the induced damping the less accurate is the approximated solution.

\subsection{The Eigenvalue Problem in viscoelastic structures}

\subsubsection{The Set of Eigenvalues}

Consider the undamped eigenvalue problem associated with the general nonlinear problem given by Eq. (5.2)

$$
\left[s^{2} \mathbf{M}+\mathbf{K}\right] \mathbf{u}_{0}=\mathbf{0}
$$

As well known, the eigenvalues of the previous problem are the complex numbers $\left\{i \omega_{j},-i \omega_{j}\right\}_{j=1}^{N}$, where $\omega_{j}$ are the natural frequencies. The mass-normalized undamped eigenvectors $\left\{\boldsymbol{\phi}_{j}\right\}_{j=1}^{N} \in \mathbb{R}^{N}$ verify the classical orthogonal relations

$$
\phi_{j}^{T} \mathbf{M} \phi_{k}=\delta_{j k} ; \quad \phi_{j}^{T} \mathbf{K} \phi_{k}=\delta_{j k} \omega_{j}^{2}
$$

where $\delta_{j k}$ represents the Kronecker delta and the superscript $(\cdot)^{T}$ transpose. Let denote by $\boldsymbol{\Phi}=\left[\phi_{1}, \ldots, \phi_{N}\right]$ the matrix whose columns are the undamped eigenvectors. Introducing the change of variable $\mathbf{u}_{0}=\boldsymbol{\Phi} \mathbf{q}_{0}$ in Eq. (5.2) and pre-multiplying the resulting equation by $\boldsymbol{\Phi}^{T}$, the nonlinear eigenvalue problem takes the form

$$
\left[s^{2} \mathbf{I}_{N}+s \boldsymbol{\Gamma}(s)+\boldsymbol{\Lambda}\right] \mathbf{q}_{0} \equiv \mathbf{D}(s) \mathbf{q}_{0}=\mathbf{0}
$$

where $\mathbf{D}(s) \in \mathbb{C}^{N \times N}$ is the Dynamic Stiffness Matrix in the modal space, $\boldsymbol{\Lambda}=$ $\operatorname{diag}\left[\omega_{j}^{2}\right]$ contains the squares of the undamped frequencies and the damping matrix in the modal space is

$$
\boldsymbol{\Phi}^{T} \mathbf{G}(s) \boldsymbol{\Phi}=\boldsymbol{\Gamma}(s)
$$


The eigenvalues are the roots of the characteristic equation given by the determinant

$$
\mathcal{D}(s)=\operatorname{det}[\mathbf{D}(s)]=0
$$

If Eq. (5.2) has normal modes, the three dynamic matrices involved in the system become diagonal in the same space. In such case, the elements of the damping matrix verify $\Gamma_{j k}(s)=0, j \neq k$ and the determinant can be expressed in the form

$$
\mathcal{D}(s)=\prod_{j=1}^{N}\left[s^{2}+s \Gamma_{j j}(s)+\omega_{j}^{2}\right]=\prod_{j=1}^{N} \mathcal{D}_{j}(s)=0
$$

For this case, the damping matrix $\mathcal{G}(t)$ is said to be proportional. Necessary and sufficient conditions for the existence of normal modes in non-viscous systems have been studied by Adhikari [29]. Any other system is characterized by complex modes and the previous decoupling is not applicable. However, if the matrix $\boldsymbol{\Gamma}(s)$ is diagonally dominant the system can be considered lightly non-proportional, and the $j$ th eigenvalue may be approximated by the the root of $\mathcal{D}_{j}(s)=0$. The method proposed in this paper differentiates between systems with proportional and non-proportional damping, see section 5.3.

Assuming that the general damping function $\mathcal{G}(t)$ induces a dissipative motion, it can be demonstrated $[64,74]$ that the viscoelastic system has $2 N+q$ eigenvalues, $q \geq 0$, ordered as

$$
\left\{\lambda_{j}, \lambda_{j}^{*}\right\}_{j=1}^{N} \cup\left\{\sigma_{j}\right\}_{j=1}^{q}
$$

Symbols $\lambda_{j}, \lambda_{j}^{*}, 1 \leq j \leq N$ represent $N$ pairs of complex-conjugate numbers. The modes associated with these eigenvalues are oscillatory with exponentially decreasing amplitude. The rest $\left\{\sigma_{j}\right\}_{j=1}^{q}$ are the so-called non-viscous eigenvalues [28], negative real numbers and therefore, associated with over-damped modes. In general, the effect of the non-viscous modes in the response of the system is not relevant and they may be neglected in the analysis [30]. The current method is exclusively focused on the computation of the complex eigenvalues.

\subsubsection{Parametric formulation of the Eigenvalues}

In viscoelastic models, energy dissipation mechanisms are governed by a set of parameters included in the damping matrix. Thus, this matrix can be considered as a multivariable function $\mathbf{G}=\mathbf{G}\left(s, \theta_{1}, \ldots, \theta_{p}\right)$, where $\theta_{1}, \ldots, \theta_{p}$ are damping parameters. For the purposes of the present paper, these $p$ parameters does not necessarily fill the complete set of damping parameters. Under this assumption, the nonlinear eigenvalue problem can be expressed as

$$
\left[s^{2} \mathbf{M}+s \mathbf{G}\left(s, \theta_{1}, \ldots, \theta_{p}\right)+\mathbf{K}\right] \mathbf{u}_{0}=\mathbf{0}
$$


whence the $j$ th complex eigenvalue $\lambda_{j}$ is also a function of the parametric array $\theta_{1}, \ldots, \theta_{p}$. The analytical computation of $\lambda_{j}=\lambda_{j}\left(\theta_{1}, \ldots, \theta_{p}\right)$ is in general not possible; however, a suitable particular choice of the damping parameters $\left(\theta_{10}, \ldots, \theta_{p 0}\right) \in \mathbb{R}^{p}$ always can lead to a null (or $s$-independent) damping matrix, so that the eigenvalue problem

$$
\left[s^{2} \mathbf{M}+s \mathbf{G}\left(s, \theta_{10}, \ldots, \theta_{p 0}\right)+\mathbf{K}\right] \mathbf{u}_{0}=\mathbf{0}
$$

becomes linear (undamped problem) or quadratic (viscous problem). For these problems the eigenvalues, named $\lambda_{j}^{0}=\lambda_{j}\left(\theta_{10}, \ldots, \theta_{p 0}\right)$ in accordance with the new functional notation, can be calculated using very efficient computational tools. Hereinafter, the damping parameters are ordered in a $p$-dimensional point $\boldsymbol{\theta}=\left\{\theta_{1}, \ldots, \theta_{p}\right\} \in \mathbb{R}^{p}$ and the damping and dynamic stiffness matrices will be noted as $\mathbf{G}(s, \boldsymbol{\theta})$ and $\mathbf{D}(s, \boldsymbol{\theta})$, respectively.

\subsection{Eigenvalues' Differential Equations}

Under the assumptions presented in the previous section, the complex eigenvalues $\lambda_{j}=\lambda_{j}(\boldsymbol{\theta})$ of the problem presented in Eq. (5.5) are multivariable functions of the parametric array $\boldsymbol{\theta} \in \mathbb{R}^{p}$. Moreover, for the $j$ th eigenvalue, the eigenvector $\mathbf{q}_{j}=\mathbf{q}_{j}(\boldsymbol{\theta})$ associated to point $\boldsymbol{\theta}$ can be computed. Thus, evaluating dynamic stiffness matrix at $s=\lambda_{j}(\boldsymbol{\theta})$ the the following equality holds for $1 \leq j \leq N$ and for any value of $\boldsymbol{\theta}$

$$
\mathbf{D}\left(\lambda_{j}(\boldsymbol{\theta}), \boldsymbol{\theta}\right) \mathbf{q}_{j}(\boldsymbol{\theta})=\mathbf{0}
$$

In this section, the principles of the proposed numerical method are developed. The method can be applied to systems with proportional as well as with nonproportional damping; both types are discussed in the different subsections.

Some remarks must be pointed in reference to the notation: the pair $(s, \boldsymbol{\theta})$ is used to represent any point in the space $\mathbb{C} \times \mathbb{R}^{p}$, that is, $s$ and $\boldsymbol{\theta}$ are assumed to be independent of each other. The notation $\lambda_{j}(\boldsymbol{\theta})$ is used for the complex eigenvalues of the damped problem that is governed by $\mathbf{G}(s, \boldsymbol{\theta})$. In some expressions, and if they are not confusing, $\left(\lambda_{j}, \boldsymbol{\theta}\right)$ will be used instead of $\left(\lambda_{j}(\boldsymbol{\theta}), \boldsymbol{\theta}\right)$. The function $\lambda_{j}(\boldsymbol{\theta})$ evaluated at $\boldsymbol{\theta}=\boldsymbol{\theta}_{0}$ is denoted by $\lambda_{j}^{0}$. Finally, $\omega_{j}$ represents the $j$ th natural eigenfrequency of the undamped problem given by Eq. (5.3). 


\subsubsection{Proportional Damping}

As described, for a multi-dof system with proportional damping the characteristic equation can be decoupled into $N$ modal equations. The damping matrix $\boldsymbol{\Gamma}(s, \boldsymbol{\theta})$ in the modal space of the undamped problem is then diagonal, and the $j$ th equation can be rewritten as

$$
\mathcal{D}_{j}\left(\lambda_{j}(\boldsymbol{\theta}), \boldsymbol{\theta}\right)=\lambda_{j}^{2}(\boldsymbol{\theta})+\lambda_{j}(\boldsymbol{\theta}) \Gamma_{j j}\left(\lambda_{j}(\boldsymbol{\theta}), \boldsymbol{\theta}\right)+\omega_{j}^{2}=0
$$

where the complex eigenvalue $\lambda_{j}(\boldsymbol{\theta}): \mathbb{R}^{p} \rightarrow \mathbb{C}$ is implicitly defined. As known, the previous statement is true if the necessary conditions of the implicit function theorem are satisfied.

Let $\boldsymbol{\theta}_{0}=\left(\theta_{10}, \ldots, \theta_{p 0}\right)$ be a certain point in the parametric domain and let $\lambda_{j}^{0}=$ $\lambda_{j}\left(\boldsymbol{\theta}_{0}\right)$ be its associated eigenvalue. As described in Section 5.2.2, the choice of $\boldsymbol{\theta}_{0}$ allows the computation of $\lambda_{j}^{0}$ from a easily-to-solve eigenvalue problem, say linear or quadratic. The function $\mathcal{D}_{j}(s, \boldsymbol{\theta}): \mathbb{C} \times \mathbb{R}^{p} \rightarrow \mathbb{C}$ vanishes at the point $\left(\lambda_{j}^{0}, \boldsymbol{\theta}_{0}\right)$. Assuming that this function is continuously differentiable in an open neighborhood of $\left(\lambda_{j}^{0}, \boldsymbol{\theta}_{0}\right)$ and that

$$
\frac{\partial \mathcal{D}_{j}\left(\lambda_{j}^{0}, \boldsymbol{\theta}_{0}\right)}{\partial s}=\Gamma_{j j}\left(\lambda_{j}^{0}, \boldsymbol{\theta}_{0}\right)+\lambda_{j}^{0}\left[2+\frac{\partial \Gamma_{j j}\left(\lambda_{j}^{0}, \boldsymbol{\theta}_{0}\right)}{\partial s}\right] \neq 0
$$

the implicit function theorem assures that there exist two positive real numbers $r>0, h>0$ and a unique continuously differentiable function $\lambda_{j}(\boldsymbol{\theta}): \mathbb{R}^{p} \rightarrow \mathbb{C}$, such that

$$
\left\{\left(\lambda_{j}(\boldsymbol{\theta}), \boldsymbol{\theta}\right): \boldsymbol{\theta} \in U\right\}=\left\{(s, \boldsymbol{\theta}) \in V_{j} \times U: \mathcal{D}_{j}(s, \boldsymbol{\theta})=0\right\}
$$

where

$$
\begin{aligned}
U=\mathcal{B}\left(\boldsymbol{\theta}_{0}, h\right) & =\left\{\boldsymbol{\theta} \in \mathbb{R}^{p}:\left\|\boldsymbol{\theta}-\boldsymbol{\theta}_{0}\right\|<h\right\} \\
V_{j}=\mathcal{B}\left(\lambda_{j}^{0}, r\right) & =\left\{z \in \mathbb{C}:\left|s-\lambda_{j}^{0}\right|<r\right\}
\end{aligned}
$$

are the open balls centered in points $\boldsymbol{\theta}_{0}$ and $\lambda_{j}^{0}$, respectively. An approximated expression of the function $\lambda_{j}(\boldsymbol{\theta})$ can obtained through expansion in its Taylor series around the point $\boldsymbol{\theta}=\boldsymbol{\theta}_{0}$. Thus, if $\boldsymbol{\theta} \in U$ then

$$
\lambda_{j}(\boldsymbol{\theta})=\lambda_{j}^{0}+\left(\boldsymbol{\theta}-\boldsymbol{\theta}_{0}\right)^{T} \boldsymbol{\nabla} \lambda_{j}^{0}+\mathcal{O}\left(h^{2}\right) \equiv \tilde{\lambda}_{j}(\boldsymbol{\theta})+\mathcal{O}\left(h^{2}\right)
$$

where $\lambda_{j}^{0} \equiv \lambda_{j}\left(\boldsymbol{\theta}_{0}\right)$ and

$$
\nabla \lambda_{j}(\boldsymbol{\theta})=\left\{\frac{\partial \lambda_{j}}{\partial \theta_{1}}, \ldots, \frac{\partial \lambda_{j}}{\partial \theta_{p}}\right\}^{T}
$$


is the gradient of $\lambda_{j}(\boldsymbol{\theta})$. An explicit expression of $\boldsymbol{\nabla} \lambda_{j}(\boldsymbol{\theta})$ can be calculated just applying the $\boldsymbol{\nabla}$-operator to the characteristic Eq. (5.13), obtaining

$$
2 \lambda_{j} \nabla \lambda_{j}+\nabla \lambda_{j} \Gamma_{j j}+\lambda_{j}\left(\frac{\partial \Gamma_{j j}\left(\lambda_{j}, \boldsymbol{\theta}\right)}{\partial s} \nabla \lambda_{j}+\nabla \Gamma_{j j}\right)=0
$$

whence, solving for the gradient of the $j$ th eigenvalue

$$
\nabla \lambda_{j}=\frac{-\lambda_{j} \nabla \Gamma_{j j}\left(\lambda_{j}, \boldsymbol{\theta}\right)}{\Gamma_{j j}\left(\lambda_{j}, \boldsymbol{\theta}\right)+\lambda_{j}\left[2+\frac{\partial \Gamma_{j j}\left(\lambda_{j}, \boldsymbol{\theta}\right)}{\partial s}\right]} \equiv-\lambda_{j} \Psi_{j}\left(\lambda_{j}, \boldsymbol{\theta}\right)
$$

Due to Eq. (5.14), the denominator does not vanish for any $\boldsymbol{\theta} \in U$. The evaluation of the gradient at point $\left(\lambda_{0}, \boldsymbol{\theta}_{0}\right)$ is required to compute the first-order approximation $\tilde{\lambda}_{j}(\boldsymbol{\theta})$ from Eq. (5.17). As well known, the approximation of the function $\lambda_{j}(\boldsymbol{\theta})$ with a Taylor linear expansion in the neighborhood of $U$ produces an error of order $\mathcal{O}\left(h^{2}\right)$. The main objective of this paper is to propose in this section a new function $\hat{\lambda}_{j}(\boldsymbol{\theta})$ that will improve the error order to $\mathcal{O}\left(h^{3}\right)$.

Let $\alpha(\xi):[0,1] \rightarrow[0,1]$ be a continuously differentiable and injective curve such that $\alpha(0)=0, \alpha(1)=1$. The set of points in the parametric domain that lies in the linear segment between $\boldsymbol{\theta}_{0}$ and a generic $\boldsymbol{\theta}$ can be expressed for $0 \leq \xi \leq 1$ as

$$
\boldsymbol{\vartheta}(\xi)=\boldsymbol{\theta}_{0}+\alpha(\xi)\left(\boldsymbol{\theta}-\boldsymbol{\theta}_{0}\right)
$$

The eigenvalue for any value of the parameter $\boldsymbol{\vartheta}(\xi) \in \mathbb{R}^{p}$, can be written as $\lambda_{j}(\xi)=\lambda_{j}(\boldsymbol{\vartheta}(\xi))$ and considered as a function of the single real variable $\xi$. The derivative of the eigenvalue with respect to $\xi$ can then be carried out using the chain rule

$$
\frac{\mathrm{d} \lambda_{j}}{\mathrm{~d} \xi}=\nabla^{T} \lambda_{j} \cdot \frac{\mathrm{d} \vartheta}{\mathrm{d} \xi}
$$

Using now the expression of the gradient calculated in Eq. (5.20) results in

$$
\frac{\mathrm{d} \lambda_{j}}{\mathrm{~d} \xi}=-\lambda_{j} \boldsymbol{\Psi}_{j}^{T}\left(\lambda_{j}(\xi), \boldsymbol{\vartheta}(\xi)\right) \cdot\left(\boldsymbol{\theta}-\boldsymbol{\theta}_{0}\right) \alpha^{\prime}(\xi)
$$

In what follows, the scalar product notation between vectors will be omitted for clarity. With the value $\boldsymbol{\theta}=\boldsymbol{\vartheta}(1)$ fixed, the previous equation is a nonlinear ordinary differential equation to be solved for function $\lambda_{j}(\xi)$. The initial-value problem must be completed with the initial value given by $\lambda_{j}(0)=\lambda_{j}^{0}$. Obviously, the exact solution is given by Eq. (5.13) in implicit form, but the challenge is to transform Eq. (5.23) into another of separated variables, whose solution can be considered a good approximation of the exact one. To this end, the following approach is proposed

$$
\boldsymbol{\Psi}_{j}\left(\lambda_{j}(\xi), \boldsymbol{\vartheta}(\xi)\right) \approx \boldsymbol{\Psi}_{j}\left(\tilde{\lambda}_{j}(\xi), \boldsymbol{\vartheta}(\xi)\right)
$$


where $\tilde{\lambda}_{j}(\xi)$ is the first-order approximation of the eigenvalue in the neighborhood of point $\boldsymbol{\theta}_{0}$. The approximation is reasonable under the hypothesis of low viscoelasticity: the function $\boldsymbol{\Psi}_{j}(s, \boldsymbol{\theta})$ defined in Eq. (5.20) depends on the viscoelastic function $\Gamma_{j j}$ and its derivative $\partial \Gamma_{j j} / \partial s$. Mathematically, the quantification of viscoelasticity in the system is directly related to the variation of the damping function $\mathbf{G}(s, \boldsymbol{\theta})$ with respect to $s[28,71]$. Hence, using the small viscoelasticity assumption, it is expected that $\Gamma_{j j}(s, \boldsymbol{\theta})$ does not present strong variations, so that the use of the linear approximation $\tilde{\lambda}_{j}(\xi)$, instead of the exact value in $\boldsymbol{\Psi}_{j}(s, \boldsymbol{\theta})$ does not induce a significant error, specially around the initial point $\boldsymbol{\theta}_{0}(\xi=0)$. This approach allows to transform the general differential Eq. (5.23) into

$$
\frac{\mathrm{d} \lambda_{j}}{\mathrm{~d} \xi}=-\lambda_{j}\left[\left(\boldsymbol{\theta}-\boldsymbol{\theta}_{0}\right)^{T} \mathbf{f}_{j}(\xi)\right]
$$

where now the one-variable function

$$
\mathbf{f}_{j}(\xi)=\boldsymbol{\Psi}_{j}\left(\tilde{\lambda}_{j}(\xi), \boldsymbol{\vartheta}(\xi)\right) \alpha^{\prime}(\xi)
$$

can be computed analytically and the initial-value differential Eq. (5.25) can be directly integrated. The solution of this equation will be denoted by $\hat{\lambda}_{j}(\xi)$ and represents the main contribution of this paper. Its closed expression for proportional damping has the form

$$
\hat{\lambda}_{j}(\xi)=\lambda_{j}^{0} \exp \left\{-\left(\boldsymbol{\theta}-\boldsymbol{\theta}_{0}\right)^{T} \mathbf{x}_{j}(\xi)\right\}
$$

in which

$$
\mathbf{x}_{j}(\xi)=\int_{0}^{\xi} \mathbf{f}_{j}(\eta) \mathrm{d} \eta
$$

may be calculated for each value of $\xi \in[0,1]$ by simple numerical quadrature. In particular, for $\xi=1$ the previous developments give the approximated expression to compute the eigenvalue at the point $\boldsymbol{\theta}$ of the parametric domain

$$
\hat{\lambda}_{j}(\boldsymbol{\theta})=\lambda_{j}^{0} \exp \left\{-\left(\boldsymbol{\theta}-\boldsymbol{\theta}_{0}\right)^{T} \mathbf{x}_{j}(1)\right\}
$$

\subsubsection{Non-proportional Damping}

The nature of non-proportional damping systems forces variations in the method from the previous section, although essentially the fundamentals are the same as those of proportional damping systems. When the hypothesis of proportionality in the damping matrix is no longer valid, the eigenproblem must be considered in its general form. The eigenrelations from Eq. (5.13) must be expressed as

$$
\mathbf{D}\left(\lambda_{j}, \boldsymbol{\theta}\right) \mathbf{q}_{j}=\left[\lambda_{j}^{2} \mathbf{I}_{N}+\lambda_{j} \boldsymbol{\Gamma}\left(\lambda_{j}, \boldsymbol{\theta}\right)+\boldsymbol{\Lambda}\right] \mathbf{q}_{j}=\mathbf{0}
$$

where $\left\{\lambda_{j}=\lambda_{j}(\boldsymbol{\theta})\right\}_{j=1}^{N}$ and $\left\{\mathbf{q}_{j}=\mathbf{q}_{j}(\boldsymbol{\theta})\right\}_{j=1}^{N}$ are the complex eigenvalues and eigenvectors, respectively, expressed as functions of the parameters $\boldsymbol{\theta} \in \mathbb{R}^{p}$. The 
determinant of the dynamic stiffenss matrix vanishes when it is evaluated in the eigenvalues for $1 \leq j \leq N$

$$
\mathcal{D}\left(\lambda_{j}(\boldsymbol{\theta}), \boldsymbol{\theta}\right)=\operatorname{det}\left[\mathbf{D}\left(\lambda_{j}(\boldsymbol{\theta}), \boldsymbol{\theta}\right)\right]=0
$$

As in the previous section, Eq. (5.31) defines the function $\lambda_{j}(\boldsymbol{\theta}): \mathbb{R}^{p} \rightarrow \mathbb{C}$ in implicit form provided that the necessary conditions of the implicit function theorem are satisfied. Let $\boldsymbol{\theta}=\boldsymbol{\theta}_{0}$ be a suitable point in the parametric domain such that the eigenvalues $\lambda_{j}^{0}=\lambda_{j}\left(\boldsymbol{\theta}_{0}\right)$ for $1 \leq j \leq N$ can be calculated from a linear or a quadratic eigenproblem (see section 5.2.2). The function $\mathcal{D}(s, \boldsymbol{\theta}): \mathbb{C} \times \mathbb{R}^{p} \rightarrow$ $\mathbb{C}$ vanishes at the point $\left(\lambda_{j}^{0}, \boldsymbol{\theta}_{0}\right)$. Assuming that this function is continuously differentiable in the open neighborhoods of $\lambda_{j}^{0}$ and $\boldsymbol{\theta}_{0}$ and that for $1 \leq j \leq N$

$$
\frac{\partial \mathcal{D}\left(\lambda_{j}^{0}, \boldsymbol{\theta}_{0}\right)}{\partial s} \neq 0
$$

then the implicit function theorem can be applied assuring that there exist two positive real numbers $r>0, h>0$ and $N$ unique continuously differentiable functions $\lambda_{j}(\boldsymbol{\theta}): \mathbb{R}^{p} \rightarrow \mathbb{C}$ such that

$$
\left\{\left(\lambda_{j}(\boldsymbol{\theta}), \boldsymbol{\theta}\right): \boldsymbol{\theta} \in U\right\}=\left\{(s, \boldsymbol{\theta}) \in V_{j} \times U: \mathcal{D}(s, \boldsymbol{\theta})=0\right\}
$$

where

$$
\begin{aligned}
U=\mathcal{B}\left(\boldsymbol{\theta}_{0}, h\right) & =\left\{\boldsymbol{\theta} \in \mathbb{R}^{p}:\left\|\boldsymbol{\theta}-\boldsymbol{\theta}_{0}\right\|<h\right\} \\
V_{j}=\mathcal{B}\left(\lambda_{j}^{0}, r\right) & =\left\{z \in \mathbb{C}:\left|s-\lambda_{j}^{0}\right|<r\right\}
\end{aligned}
$$

are the open balls centered in points $\boldsymbol{\theta}_{0}$ and $\lambda_{j}^{0}$ for $1 \leq j \leq N$. As before, the first-order approximation of the Taylor series may be performed provided that the gradient of the eigenvalue is available at the point $\boldsymbol{\theta}=\boldsymbol{\theta}_{0}$. Thus, if $\boldsymbol{\theta} \in U$

$$
\lambda_{j}(\boldsymbol{\theta})=\lambda_{j}^{0}+\left(\boldsymbol{\theta}-\boldsymbol{\theta}_{0}\right)^{T} \boldsymbol{\nabla} \lambda_{j}^{0}+\mathcal{O}\left(h^{2}\right) \equiv \tilde{\lambda}_{j}(\boldsymbol{\theta})+\mathcal{O}\left(h^{2}\right)
$$

Now, the expression of $\boldsymbol{\nabla} \lambda_{j}(\boldsymbol{\theta})$ may be obtained taking derivatives with respect to the parameters of the characteristic Eq. (5.31) and applying the chain rule

$$
\frac{\partial \mathcal{D}\left(\lambda_{j}, \boldsymbol{\theta}\right)}{\partial s} \nabla \lambda_{j}+\nabla \mathcal{D}\left(\lambda_{j}, \boldsymbol{\theta}\right)=0
$$

and hence, the gradient of the eigenvalue can be expressed as function of the derivatives of the determinant as

$$
\nabla \lambda_{j}=-\frac{\nabla \mathcal{D}\left(\lambda_{j}, \boldsymbol{\theta}\right)}{\frac{\partial \mathcal{D}\left(\lambda_{j}, \boldsymbol{\theta}\right)}{\partial s}}
$$

There is another way to obtain these derivatives; Adhikari [59] developed a method for non-viscous systems to find the derivative of the $j$ th eigenvalue with respect 
to certain design parameter. Let $\lambda_{j}=\lambda_{j}(\boldsymbol{\theta}), \mathbf{q}_{j}=\mathbf{q}_{j}(\boldsymbol{\theta})$ be the $j$ th eigenvalue and its associated eigenvector, for a certain value of the parameters $\boldsymbol{\theta}$. According to the reference, the derivatives can alternatively be expressed as

$$
\nabla \lambda_{j}=-\frac{\mathbf{q}_{j}^{T} \nabla \mathbf{D}\left(\lambda_{j}, \boldsymbol{\theta}\right) \mathbf{q}_{j}}{\mathbf{q}_{j}^{T} \frac{\partial \mathbf{D}\left(\lambda_{j}, \boldsymbol{\theta}\right)}{\partial s} \mathbf{q}_{j}}
$$

where

$$
\mathbf{q}_{j}^{T} \boldsymbol{\nabla} \mathbf{D} \mathbf{q}_{j}=\left\{\mathbf{q}_{j}^{T} \frac{\partial \mathbf{D}}{\partial \theta_{1}} \mathbf{q}_{j}, \ldots, \mathbf{q}_{j}^{T} \frac{\partial \mathbf{D}}{\partial \theta_{p}} \mathbf{q}_{j}\right\}^{T}
$$

Just as in the case of proportional damping, the expression of the gradient of the $j$ th eigenvalue is the starting point of the proposed method. Here, two different expressions of the derivatives are available from Eqs. (5.37) and (5.38). Consequently two variants are possible, related to the use of the expression of the eigenvalue derivatives derived in terms of the stiffness matrix determinant -DETD method-, or with the use of the dynamic stiffness matrix and the eigenvectors $\mathbf{q}_{j}$ -EIGD method-.

\section{Method based on the determinant of the dynamic stiffness matrix}

The function $\mathcal{D}(s, \boldsymbol{\theta})$ is the determinant of the dynamic stiffness matrix. Its general expression in the case of non-proportional damping systems, for any value $s \in \mathbb{C}$ and for any array of parameters $\boldsymbol{\theta} \in \mathbb{R}^{p}$, is

$$
\mathcal{D}(s, \boldsymbol{\theta})=\operatorname{det}\left[s^{2} \mathbf{I}_{N}+s \mathbf{G}(s, \boldsymbol{\theta})+\boldsymbol{\Lambda}\right]
$$

For the undamped problem, the expression of the determinant of the dynamic stiffness matrix $\mathcal{D}_{u}(s)$ is

$$
\mathcal{D}_{u}(s)=\operatorname{det}\left[s^{2} \mathbf{I}_{N}+\boldsymbol{\Lambda}\right]=\prod_{j=1}^{N}\left(s^{2}+\omega_{j}^{2}\right)
$$

Obviously this function does not depend on the damping parameters. Evaluating both determinants at $s=0$

$$
\mathcal{D}(0, \boldsymbol{\theta})=\prod_{j=1}^{N} \omega_{j}^{2}=D_{u}(0)
$$

Consequently, it can be assumed that there exists a function $\mathcal{J}(s, \boldsymbol{\theta})$ such that

$$
\mathcal{D}(s, \boldsymbol{\theta})=\mathcal{D}_{u}(s)+s \mathcal{J}(s, \boldsymbol{\theta})
$$


Since the function $\mathcal{D}_{u}(s)$ does not depend on the array of damping parameters, the gradient can be directly written as

$$
\boldsymbol{\nabla D}(s, \boldsymbol{\theta})=s \boldsymbol{\nabla} \mathcal{J}(s, \boldsymbol{\theta})
$$

Introducing now this expression in Eq. (5.37), the gradient of the $j$ th eigenvalue can be expressed as

$$
\nabla \lambda_{j}=-\lambda_{j} \frac{\nabla \mathcal{J}\left(\lambda_{j}, \boldsymbol{\theta}\right)}{\frac{\partial \mathcal{D}\left(\lambda_{j}, \boldsymbol{\theta}\right)}{\partial s}} \equiv-\lambda_{j} \boldsymbol{\Psi}_{j}\left(\lambda_{j}, \boldsymbol{\theta}\right)
$$

Due to the similarities, the same symbol $\boldsymbol{\Psi}_{j}(s, \boldsymbol{\theta})$ as in proportional damping systems has been used, although the expression is different. Therefore, the gradient in Eq. (5.45) has exactly the same structure as that of Eq. (5.20). These correspondences allow the applying of the same mathematical development as before to obtain the function $\hat{\lambda}_{j}(\boldsymbol{\theta})$, through the integration of the ordinary differential Eq. (5.25). The evaluation of the new $\boldsymbol{\Psi}_{j}(s, \boldsymbol{\theta})$ requires the computation of the partial derivative of a determinant, computationally expensive for relatively large systems. For these cases, the partial derivatives may be numerically calculated by means of finite differences.

\section{Method based on the eigenvectors approximation}

The method developed in this subsection uses the expression of the eigenvalue derivatives given by Eq. (5.38). This method requires the estimation of the associated eigenvector, therefore it is named EIGD. The partial derivatives with respect to the damping parameters and also with respect to $s$ can be calculated. Rewriting the dynamic stiffness matrix in its general form as function of the array $\boldsymbol{\theta}$

$$
\mathbf{D}(s, \boldsymbol{\theta})=s^{2} \mathbf{I}_{N}+s \boldsymbol{\Gamma}(s, \boldsymbol{\theta})+\boldsymbol{\Lambda}
$$

with which the partial derivatives will be

$$
\begin{aligned}
\nabla \mathbf{D}(s, \boldsymbol{\theta}) & =s \boldsymbol{\nabla} \boldsymbol{\Gamma}(s, \boldsymbol{\theta}) \\
\frac{\partial \mathbf{D}}{\partial s} & =\boldsymbol{\Gamma}(s, \boldsymbol{\theta})+s\left(2 \mathbf{I}_{N}+\frac{\partial \boldsymbol{\Gamma}}{\partial s}\right)
\end{aligned}
$$

Substituting the previous expressions in Eq. (5.38) an equation with structure

$$
\nabla \lambda_{j}=-\lambda_{j} \Psi_{j}\left(\lambda_{j}, \boldsymbol{\theta}\right)
$$

is again obtained, for which now

$$
\boldsymbol{\Psi}_{j}\left(\lambda_{j}, \boldsymbol{\theta}\right)=\frac{\mathbf{q}_{j}^{T} \nabla \boldsymbol{\Gamma}\left(\lambda_{j}, \boldsymbol{\theta}\right) \mathbf{q}_{j}}{\mathbf{q}_{j}^{T}\left[\boldsymbol{\Gamma}\left(\lambda_{j}, \boldsymbol{\theta}\right)+\lambda_{j}\left(2 \mathbf{I}_{N}+\frac{\partial \boldsymbol{\Gamma}\left(\lambda_{j}, \boldsymbol{\theta}\right)}{\partial s}\right)\right] \mathbf{q}_{j}}
$$


In order to implement EIGD, it will be necessary to approximately estimate the eigenvector $\mathbf{q}_{j}$. For that, several steps have to be carried out. Consider the linear eigenvalue problem of the dynamic stiffness matrix

$$
\left[\mathbf{D}(s, \boldsymbol{\theta})-\nu(s) \mathbf{I}_{N}\right] \mathbf{w}(s)=\mathbf{0}
$$

where $\nu, \mathbf{w}$ represent the eigensolution associated with value $s \in \mathbb{C}$. Adhikari [28] used this eigenvalue problem to describe the theoretical basis of the non-viscously damped systems. The reference demonstrated that evaluating $s=\lambda_{j}(\boldsymbol{\theta})$ on the linear eigenvalue problem Eq. (5.50), there exists an eigensolution given by $\nu_{j}, \mathbf{w}_{j}$ -without loss of generality it can be denoted as the $j$ th eigensolution-, such that

$$
\nu_{j}\left(\lambda_{j}(\boldsymbol{\theta})\right)=0, \quad \mathbf{w}_{j}\left(\lambda_{j}(\boldsymbol{\theta})\right)=\mathbf{q}_{j}
$$

As shown in Eq. (5.17), the difference between the exact and the linear approximation of the complex eigenvalues around $\boldsymbol{\theta}=\boldsymbol{\theta}_{0}$ can be bounded by

$$
\left|\tilde{\lambda}_{j}(\boldsymbol{\theta})-\lambda_{j}(\boldsymbol{\theta})\right| \leq r_{j} h^{2}
$$

for some positive real number $r_{j}>0$. $h$ is the radius of the open ball $U=\mathcal{B}\left(\boldsymbol{\theta}_{0}, h\right)$ defined in Eq. (5.34). Since $\tilde{\lambda}_{j}(\boldsymbol{\theta})$ is an approximation of the exact eigenvalue, then $\nu_{j}\left(\tilde{\lambda}_{j}(\boldsymbol{\theta})\right) \neq 0$. But it is expected that $\left|\nu_{j}\left(\tilde{\lambda}_{j}(\boldsymbol{\theta})\right)\right|<<1$ in the neighborhood $U$ of the initial point $\boldsymbol{\theta}_{0}$; In fact $\nu_{j}\left(\lambda_{j}^{0}\right)=0$ is verified. Taking into account the bound given by Eq. (5.53), $\left|\nu_{j}\left(\tilde{\lambda}_{j}(\boldsymbol{\theta})\right)\right|$ may also be bounded in terms of $h$. Indeed, assuming that $\nu(s)$ is Lipschitz continuous with constant $c_{j}$, then $\forall \boldsymbol{\theta} \in U$

$$
\begin{aligned}
\left|\nu_{j}\left(\tilde{\lambda}_{j}(\boldsymbol{\theta})\right)\right| & =\left|\nu_{j}\left(\tilde{\lambda}_{j}(\boldsymbol{\theta})\right)-\nu_{j}\left(\lambda_{j}(\boldsymbol{\theta})\right)\right| \\
& \leq c_{j}\left|\tilde{\lambda}_{j}(\boldsymbol{\theta})-\lambda_{j}(\boldsymbol{\theta})\right| \leq c_{j} r_{j} h^{2}
\end{aligned}
$$

These arguments lead to propose an approach to obtain the vector $\mathbf{q}_{j}$ for Eq. (5.49). Let $\tilde{\lambda}_{j}(\boldsymbol{\theta})$ be the linear approximation of the $j$ th complex eigenvalue for certain value of the parametric domain. It can be denoted by $a_{j}(\boldsymbol{\theta})=\nu_{j}\left(\tilde{\lambda}_{j}(\boldsymbol{\theta})\right)$ to the minimum eigenvalue (in the sense of absolute value) of matrix $\mathbf{D}\left(\tilde{\lambda}_{j}(\boldsymbol{\theta})\right)$, i.e.

$$
\left|a_{j}(\boldsymbol{\theta})\right|=\min \left\{|z|: \operatorname{det}\left[\mathbf{D}\left(\tilde{\lambda}_{j}(\boldsymbol{\theta})\right)-z \mathbf{I}_{N}\right]=0\right\}
$$

Under this conditions the $j$ th eigenvector $\mathbf{q}_{j}(\boldsymbol{\theta})$ of the eigenvalue $\lambda_{j}(\boldsymbol{\theta})$ will be approximated by the eigenvector $\tilde{\mathbf{q}}_{j}(\boldsymbol{\theta})$ associated to $a_{j}(\boldsymbol{\theta})$, that is

$$
\left[\mathbf{D}\left(\tilde{\lambda}_{j}(\boldsymbol{\theta})\right)-a_{j}(\boldsymbol{\theta}) \mathbf{I}_{N}\right] \tilde{\mathbf{q}}_{j}(\boldsymbol{\theta})=\mathbf{0}
$$


Therefore, the function $\boldsymbol{\Psi}_{j}$ will be redefined as

$$
\boldsymbol{\Psi}_{j}\left(\tilde{\lambda}_{j}(\boldsymbol{\theta}), \boldsymbol{\theta}\right)=\frac{\tilde{\mathbf{q}}_{j}^{T} \boldsymbol{\nabla} \boldsymbol{\Gamma}\left(\tilde{\lambda}_{j}, \boldsymbol{\theta}\right) \tilde{\mathbf{q}}_{j}}{\tilde{\mathbf{q}}_{j}^{T}\left[\boldsymbol{\Gamma}\left(\tilde{\lambda}_{j}, \boldsymbol{\theta}\right)+\tilde{\lambda}_{j}\left(2 \mathbf{I}_{N}+\frac{\partial \boldsymbol{\Gamma}\left(\tilde{\lambda}_{j}, \boldsymbol{\theta}\right)}{\partial s}\right)\right] \tilde{\mathbf{q}}_{j}}
$$

\subsection{Approximation of eigenvectors}

As known, the modal space of proportional damped systems equals that of the undamped, i.e. both share eigenvectors that form an orthogonal base of $\mathbb{R}^{N}$. For a non-proportional damping matrix, the eigenvectors are $N$-dimensional complex arrays. Assuming that $\hat{\lambda}_{j}(\boldsymbol{\theta})$ is the $j$ th complex eigenvalue estimated with the proposed method from Eq. (5.29), the associated complex eigenvector can be evaluated from different numerical techniques. In this section, three will be described: (i) Direct solution of the ill-conditioned linear system, (ii) Adhikari's approach for lightly non-proportional damping and (iii) First order approximation valid for $\left\|\boldsymbol{\theta}-\boldsymbol{\theta}_{0}\right\| \ll 1$, . Each depend on the obtained eigenvalue and therefore, it is expected that a better approximation of it will give better accuracy for the eigenvector computation. In this sense, and as will be shown in the numerical examples, EIGD is more suitable than DETD because in general presents lower error.

\subsubsection{Solution of the ill-conditioned linear system}

Using the same nomenclature as in Eq. (5.10), $\mathbf{u}_{j}$ denotes the $j$ th eigenvector, that can be written as a linear combination of $\phi_{1}, \ldots, \phi_{N}$ as

$$
\mathbf{u}_{j}=\sum_{k=1}^{N} a_{k}^{(j)} \phi_{k}
$$

Since any vector proportional to $\mathbf{u}_{j}$ is also an eigenvector, the value $a_{j}^{(j)}=1$ is fixed without loss of generality. The aim is to calculate the $N-1$ coefficients $a_{k}^{(j)}$ for $1 \leq k \leq N$ and $k \neq j$. Obviously, from $\mathbf{u}_{j}=\mathbf{\Phi} \mathbf{q}_{j}$, these coefficients are the components of $\mathbf{q}_{j}$, that is

$$
\begin{aligned}
\mathbf{q}_{j}^{T} & =\left\{a_{1}^{(j)}, \ldots, a_{j-1}^{(j)}, 1, a_{j+1}^{(j)}, \ldots, a_{N}^{(j)}\right\} \\
& \equiv\left\{\mathbf{a}_{1}^{(j)^{T}}, \mathbf{a}_{2}^{(j)^{T}}(=1), \mathbf{a}_{3}^{(j)^{T}}\right\}
\end{aligned}
$$

and they can be obtained solving the linear system Eq. (5.30). The eigenvalue $\hat{\lambda}_{j}(\boldsymbol{\theta})$ calculated with the proposed method is assumed to be close to the exact 
one, so that the linear system $\mathbf{D}\left(\hat{\lambda}_{j}(\boldsymbol{\theta}), \boldsymbol{\theta}\right) \mathbf{q}_{j}(\boldsymbol{\theta}) \approx \mathbf{0}$ becomes ill-conditioned. This system can be written in terms of the sub-matrices of $\mathbf{D}\left(\hat{\lambda}_{j}(\boldsymbol{\theta}), \boldsymbol{\theta}\right)$ generated from the partition of $\mathbf{q}_{j}$, resulting

$$
\left[\begin{array}{lll}
\mathbf{D}_{j 11} & \mathbf{D}_{j 12} & \mathbf{D}_{j 13} \\
\mathbf{D}_{j 21} & \mathbf{D}_{j 22} & \mathbf{D}_{j 23} \\
\mathbf{D}_{j 31} & \mathbf{D}_{j 32} & \mathbf{D}_{j 33}
\end{array}\right]\left\{\begin{array}{l}
\mathbf{a}_{1}^{(j)} \\
\mathbf{a}_{2}^{(j)} \\
\mathbf{a}_{3}^{(j)}
\end{array}\right\}=\left\{\begin{array}{l}
\mathbf{0} \\
\mathbf{0} \\
\mathbf{0}
\end{array}\right\}
$$

Hence, the coefficients $a_{j k}$ can be solved as

$$
\left\{\begin{array}{c}
\mathbf{a}_{1}^{(j)} \\
\mathbf{a}_{3}^{(j)}
\end{array}\right\}=-\left[\begin{array}{ll}
\mathbf{D}_{j 11} & \mathbf{D}_{j 13} \\
\mathbf{D}_{j 31} & \mathbf{D}_{j 33}
\end{array}\right]^{-1}\left\{\begin{array}{l}
\mathbf{D}_{j 12} \\
\mathbf{D}_{j 32}
\end{array}\right\}
$$

This method is valid for any non-proportional system and gives the exact solution for the eigenvector provided that the associated eigenvalue is exact. However, it requires the inversion of an $N-1$ order matrix. In the following two subsections more efficient approaches are described, but only valid under the assumption of light non-proportionality and under light damping.

\subsubsection{Approximation for lightly non-proportional damping}

For light non-proportional damping, approximate eigenvectors have been proposed by Adhikari [28] based on the Neumann expansion, resulting in closed-form expressions functions of the off-diagonal terms from $\boldsymbol{\Gamma}\left(\hat{\lambda}_{j}, \boldsymbol{\theta}\right)=\boldsymbol{\Phi}^{T} \mathbf{G}\left(\hat{\lambda}_{j}, \boldsymbol{\theta}\right) \boldsymbol{\Phi}$. The second order approximation of the $j$ th eigenvector can be expressed as a linear combination of the undamped eigenvectors

$$
\begin{aligned}
\mathbf{u}_{j}(\boldsymbol{\theta}) \approx \boldsymbol{\phi}_{j}-\hat{\lambda}_{j} \sum_{\substack{k=1 \\
k \neq j}}^{N} \frac{\Gamma_{k j}\left(\hat{\lambda}_{j}, \boldsymbol{\theta}\right)}{\mathcal{D}_{k}\left(\hat{\lambda}_{j}, \boldsymbol{\theta}\right)} \boldsymbol{\phi}_{k} \\
+\hat{\lambda}_{j}^{2} \sum_{\substack{k=1 \\
k \neq j}}^{N} \sum_{\substack{l=1 \\
l \neq j \neq k}}^{N} \frac{\Gamma_{k l}\left(\hat{\lambda}_{j}, \boldsymbol{\theta}\right) \Gamma_{l j}\left(\hat{\lambda}_{j}, \boldsymbol{\theta}\right)}{\mathcal{D}_{k}\left(\hat{\lambda}_{j}, \boldsymbol{\theta}\right) \mathcal{D}_{l}\left(\hat{\lambda}_{j}, \boldsymbol{\theta}\right)} \boldsymbol{\phi}_{k}
\end{aligned}
$$

where $\mathcal{D}_{j}(s, \boldsymbol{\theta})=s^{2}+s \Gamma_{j j}(s, \boldsymbol{\theta})+\omega_{j}^{2}$. Note that the above expression provides closed-forms of the coefficients $a_{k}^{(j)}$ introduced in Eq. (5.57), so that no matrixinversion is required. 


\subsubsection{Approximation for light damping}

As described in Section 5.2.2, the damping parameters can always be chosen so that from the initial value $\boldsymbol{\theta}_{0}$ the undamped problem $\mathbf{G}\left(s, \boldsymbol{\theta}_{0}\right)=\mathbf{0}$ is obtained; see for instance the numerical examples 2, 3 for non-proportional damping. For this initial value, the hypothesis of light damping is equivalent to assume that the distance between the real damping parameters and the initial values verifies $\left\|\boldsymbol{\theta}-\boldsymbol{\theta}_{0}\right\| \ll 1$. Therefore, it seems logical to approximate the complex eigenvectors with the first order Taylor expansion

$$
\mathbf{u}_{j}(\boldsymbol{\theta})=\mathbf{u}_{j}\left(\boldsymbol{\theta}_{0}\right)+\sum_{r=1}^{p} \frac{\partial \mathbf{u}_{j}\left(\boldsymbol{\theta}_{0}\right)}{\partial \theta_{r}}\left(\theta_{r}-\theta_{r 0}\right)
$$

Since $\mathbf{G}\left(s, \boldsymbol{\theta}_{0}\right)=\mathbf{0}, \forall s \in \mathbb{C}$, then $\mathbf{u}_{j}\left(\boldsymbol{\theta}_{0}\right)=\boldsymbol{\phi}_{j}$ and $\lambda_{j}\left(\boldsymbol{\theta}_{0}\right)=i \omega_{j}$. The analytical expression of the eigenvector derivative has been developed by Adhikari [59].

$$
\frac{\partial \mathbf{u}_{j}\left(\boldsymbol{\theta}_{0}\right)}{\partial \theta_{r}}=-\frac{\alpha_{j r}}{2 \gamma_{j}} \boldsymbol{\phi}_{j}-\sum_{\substack{k=1 \\ k \neq j}}^{N} \frac{\beta_{k j r} \boldsymbol{\phi}_{k}}{\gamma_{k}\left(i \omega_{j}-i \omega_{k}\right)}
$$

where the coefficients $\gamma_{j}, \alpha_{j r}, \beta_{k j r}$ for $1 \leq j, k \leq N$ and $1 \leq r \leq p$ are given by

$$
\begin{aligned}
\gamma_{j} & =\boldsymbol{\phi}_{j}^{T} \frac{\partial \mathbf{D}\left(i \omega_{j}, \boldsymbol{\theta}_{0}\right)}{\partial s} \boldsymbol{\phi}_{j}=i \omega_{j}\left(2+\frac{\partial \Gamma_{j j}\left(i \omega_{j}, \boldsymbol{\theta}_{0}\right)}{\partial s}\right) \\
\alpha_{j r} & =\boldsymbol{\phi}_{j}^{T} \frac{\partial^{2} \mathbf{D}\left(i \omega_{j}, \boldsymbol{\theta}_{0}\right)}{\partial s \partial \theta_{r}} \boldsymbol{\phi}_{j}=\frac{\partial \Gamma_{j j}\left(i \omega_{j}, \boldsymbol{\theta}_{0}\right)}{\partial s}+i \omega_{j} \frac{\partial^{2} \Gamma_{j j}\left(i \omega_{j}, \boldsymbol{\theta}_{0}\right)}{\partial s \partial \theta_{r}} \\
\beta_{k j r} & =\boldsymbol{\phi}_{k}^{T} \frac{\partial \mathbf{D}\left(i \omega_{j}, \boldsymbol{\theta}_{0}\right)}{\partial \theta_{r}} \boldsymbol{\phi}_{j}=i \omega_{j} \frac{\partial \Gamma_{k j}\left(i \omega_{j}, \boldsymbol{\theta}_{0}\right)}{\partial \theta_{r}}
\end{aligned}
$$

Notice that the obtained expressions depend explicitly on the undamped eigensolution and on the elements of the damping matrix $\boldsymbol{\Gamma}\left(i \omega_{j}, \boldsymbol{\theta}_{0}\right)$ described in modal coordinates. Therefore, the needed current algorithm effort for eigenvectors' computation is similar to that for lightly non-proportional systems.

\subsection{Analysis of the error and computational complexity}

The two methods used for non-proportional damping systems require greater computational effort than the one for proportional: EIGD needs to calculate the eigenvalue problem from Eq. (5.50) and DETD needs to evaluate the determinant to obtain its derivatives. A comparison of the computational complexity of both methods along with the error analysis of the proposed improved functions is presented in this section. 


\subsubsection{Analysis of the error}

The previous subsection focused on the methodology to build the functions that estimate the complex eigenvalues, $\hat{\lambda}_{j}(\boldsymbol{\theta})$. For both proportional and non-proportional damping, closed expressions have been developed with the general form

$$
\hat{\lambda}_{j}(\boldsymbol{\theta})=\lambda_{j}^{0} \exp \left\{-\left(\boldsymbol{\theta}-\boldsymbol{\theta}_{0}\right)^{T} \mathbf{x}_{j}\right\}
$$

where

$$
\begin{aligned}
\mathbf{x}_{j} & =\int_{0}^{1} \boldsymbol{\Psi}_{j}\left(\tilde{\lambda}_{j}(\boldsymbol{\vartheta}(\xi)), \boldsymbol{\vartheta}(\xi)\right) \mathrm{d} \xi \\
\boldsymbol{\vartheta}(\xi) & =\boldsymbol{\theta}_{0}+\left(\boldsymbol{\theta}-\boldsymbol{\theta}_{0}\right) \xi
\end{aligned}
$$

The curve $\alpha(\xi)=\xi$ has been considered to represent the set of values in the parameter domain between the points $\boldsymbol{\theta}_{0}, \boldsymbol{\theta}$. The function $\boldsymbol{\Psi}_{j}(s, \boldsymbol{\theta})$ may take any expresion from Eqs. (5.20), (5.45) or (5.49). This section is focused on obtaining the error order between improved and exact solutions, requiring to adopt some hypothesis about $\boldsymbol{\Psi}_{j}(s, \boldsymbol{\theta})$. Let $\left(\lambda_{j}^{0}, \boldsymbol{\theta}_{0}\right)$ be the initial point of the proposed method and let $U, V_{j}$ be the neighborhoods of $\left(\boldsymbol{\theta}_{0}, \lambda_{j}^{0}\right)$, defined in Eqs. (5.16), (5.34). Also assume that the function $\boldsymbol{\Psi}_{j}(s, \boldsymbol{\theta}): V_{j} \times U \rightarrow \mathbb{C}^{p}$ verifies the following properties

H1 is bounded in the set $V_{j} \times U$; thus there exists a real positive number $\kappa_{j}>0$ such that $\forall s \in V_{j}, \boldsymbol{\theta} \in U$

$$
\left\|\Psi_{j}(s, \boldsymbol{\theta})\right\| \leq \kappa_{j}
$$

H2 is Lipschitz continuous with constant $\rho_{j}>0$, with respect to the first variable $s$ in the set $B$, i.e., $\forall u, v \in V_{j}, \boldsymbol{\theta} \in U$

$$
\left\|\boldsymbol{\Psi}_{j}(z, \boldsymbol{\theta})-\boldsymbol{\Psi}_{j}(w, \boldsymbol{\theta})\right\| \leq \rho_{j}|z-w|
$$

Given a point $\boldsymbol{\theta} \in U$, the exact eigenvalue $\lambda_{j}(\boldsymbol{\theta})$ verifies

$$
\lambda_{j}(\boldsymbol{\theta})=\lambda_{j}^{0} \exp \left\{-\left(\boldsymbol{\theta}-\boldsymbol{\theta}_{0}\right)^{T} \mathbf{y}_{j}\right\}
$$

where now

$$
\mathbf{y}_{j}=\int_{0}^{1} \boldsymbol{\Psi}_{j}\left(\lambda_{j}(\boldsymbol{\vartheta}(\xi)), \boldsymbol{\vartheta}(\xi)\right) \mathrm{d} \xi
$$

The aim is to bound the expression $\left|\hat{\lambda}_{j}(\boldsymbol{\theta})-\lambda_{j}(\boldsymbol{\theta})\right|$ in terms of the radius $h>0$ of the ball $U$ centered in $\boldsymbol{\theta}_{0}$. First, the following values depending on $\boldsymbol{\theta}$

$$
A_{j}(\boldsymbol{\theta})=\left(\boldsymbol{\theta}-\boldsymbol{\theta}_{0}\right)^{T} \mathbf{x}_{j}, \quad B_{j}(\boldsymbol{\theta})=\left(\boldsymbol{\theta}-\boldsymbol{\theta}_{0}\right)^{T} \mathbf{y}_{j}
$$


can be defined. Since these functions are directly related with $\boldsymbol{\Psi}_{j}(s, \boldsymbol{\theta})$ (see Eqs. (5.66),(5.68)), then $\left|A_{j}(\boldsymbol{\theta})\right| \leq h \kappa_{j},\left|B_{j}(\boldsymbol{\theta})\right| \leq h \kappa_{j}$ hold for any $\boldsymbol{\theta} \in U$. Therefore, a bound of $\left|A_{j}^{n}(\boldsymbol{\theta})-B_{j}^{n}(\boldsymbol{\theta})\right|$ may be calculated using the expansion of the difference of $n$-order powers. In fact, for $n \geq 1$

$$
\begin{aligned}
& \left|A_{j}^{n}(\boldsymbol{\theta})-B_{j}^{n}(\boldsymbol{\theta})\right| \\
& \quad=\left|\left[A_{j}(\boldsymbol{\theta})-B_{j}(\boldsymbol{\theta})\right] \sum_{k=0}^{n} A_{j}^{n-k}(\boldsymbol{\theta}) B_{j}^{k-1}(\boldsymbol{\theta})\right| \\
& \quad \leq\left|A_{j}(\boldsymbol{\theta})-B_{j}(\boldsymbol{\theta})\right| \sum_{k=1}^{n}\left|A_{j}(\boldsymbol{\theta})\right|^{n-k}\left|B_{j}(\boldsymbol{\theta})\right|^{k-1} \\
& \quad \leq\left|A_{j}(\boldsymbol{\theta})-B_{j}(\boldsymbol{\theta})\right| \sum_{k=1}^{n}\left(\kappa_{j} h\right)^{n-k}\left(\kappa_{j} h\right)^{k-1} \\
& \quad \leq\left|A_{j}(\boldsymbol{\theta})-B_{j}(\boldsymbol{\theta})\right| n\left(\kappa_{j} h\right)^{n-1} \\
& \quad \leq\left\|\boldsymbol{\theta}-\boldsymbol{\theta}_{0}\right\|\left\|\mathbf{x}_{j}-\mathbf{y}_{j}\right\| n\left(\kappa_{j} h\right)^{n-1} \\
& \quad \leq\left\|\mathbf{x}_{j}-\mathbf{y}_{j}\right\| n h\left(\kappa_{j} h\right)^{n-1}
\end{aligned}
$$

Since the error order between the exact eigenvalue and the first-order approximation is $\mathcal{O}\left(h^{2}\right)$, there exists a real positive number $r_{j}>0$ such that $\left|\lambda_{j}(\boldsymbol{\theta})-\tilde{\lambda}_{j}(\boldsymbol{\theta})\right| \leq$ $r_{j} h^{2}$. Then, $\left\|\mathbf{x}_{j}-\mathbf{y}_{j}\right\|$ may be bounded using the Lipschitz continuity of $\boldsymbol{\Psi}_{j}(s, \boldsymbol{\theta})$

$$
\begin{aligned}
& \left\|\mathbf{x}_{j}-\mathbf{y}_{j}\right\| \\
& =\left\|\int_{0}^{1}\left[\boldsymbol{\Psi}_{j}\left(\tilde{\lambda}_{j}(\boldsymbol{\vartheta}(\xi)), \boldsymbol{\vartheta}(\xi)\right)-\boldsymbol{\Psi}_{j}\left(\lambda_{j}(\boldsymbol{\vartheta}(\xi)), \boldsymbol{\vartheta}(\xi)\right)\right] \mathrm{d} \xi\right\| \\
& \leq \int_{0}^{1}\left\|\boldsymbol{\Psi}_{j}\left(\tilde{\lambda}_{j}(\boldsymbol{\vartheta}(\xi)), \boldsymbol{\vartheta}(\xi)\right)-\boldsymbol{\Psi}_{j}\left(\lambda_{j}(\boldsymbol{\vartheta}(\xi)), \boldsymbol{\vartheta}(\xi)\right)\right\| \mathrm{d} \xi \\
& \leq \int_{0}^{1} \rho_{j}\left|\lambda_{j}(\boldsymbol{\vartheta}(\xi))-\tilde{\lambda}_{j}(\boldsymbol{\vartheta}(\xi))\right| \mathrm{d} \xi \leq \rho_{j} r_{j} h^{2}
\end{aligned}
$$


These results allow the calculation of the error between $\hat{\lambda}_{j}(\boldsymbol{\theta}), \lambda_{j}(\boldsymbol{\theta})$ using their definitions from Eqs. (5.65), (5.67)

$$
\begin{aligned}
& \mid \hat{\lambda}_{j}(\boldsymbol{\theta})-\lambda_{j}(\boldsymbol{\theta}) \mid \\
&=\left|\lambda_{j}^{0} e^{-A_{j}(\boldsymbol{\theta})}-\lambda_{j}^{0} e^{-B_{j}(\boldsymbol{\theta})}\right| \\
&=\left|\lambda_{j}^{0}\right|\left|\sum_{n=1}^{\infty} \frac{(-1)^{n}}{n !}\left[A_{j}^{n}(\boldsymbol{\theta})-B_{j}^{n}(\boldsymbol{\theta})\right]\right| \\
& \leq\left|\lambda_{j}^{0}\right| \sum_{n=1}^{\infty} \frac{1}{n !}\left|A_{j}^{n}(\boldsymbol{\theta})-B_{j}^{n}(\boldsymbol{\theta})\right| \\
& \leq\left|\lambda_{j}^{0}\right|\left\|\mathbf{x}_{j}-\mathbf{y}_{j}\right\| h \sum_{n=1}^{\infty} \frac{n\left(\kappa_{j} h\right)^{n-1}}{n !} \\
& \leq\left|\lambda_{j}^{0}\right| \rho_{j} r_{j} h^{3} \sum_{n=1}^{\infty} \frac{\left(\kappa_{j} h\right)^{n-1}}{(n-1) !} \\
& \leq\left|\lambda_{j}^{0}\right| \rho_{j} r_{j} h^{3} e^{\kappa_{j} h}
\end{aligned}
$$

The previous bounding states that the approximation order of the improved solution is $\mathcal{O}\left(h^{3}\right)$, meanwhile using the Taylor series linear approximation the order would be $\mathcal{O}\left(h^{2}\right)$. This ensures that, indeed, there is an improvement in the calculation of the eigenvalue with the proposed method.

\subsubsection{Computational Complexity}

This subsection aims at comparing the computational complexities of DETD and EIGD. When a point $\boldsymbol{\theta} \in \mathbb{R}^{p}$ is fixed, the evaluation of the function $\hat{\lambda}_{j}(\boldsymbol{\theta})$ requires the numerical integration of the vector-valued function $\boldsymbol{\Psi}_{j}\left(\tilde{\lambda}_{j}(\boldsymbol{\theta}), \boldsymbol{\theta}\right)$. Therefore, since both methods essentially differ in the form of this function, the computational differences can be established by calculating the complexity of a single evaluation.

It can be assumed that the calculation of the partial derivatives of the determinant $\mathcal{D}(s, \boldsymbol{\theta})$ are evaluated numerically with central finite differences. Hence, one partial derivative requires to compute the determinant two times; since the numerator has $p$ components, one evaluation of $\boldsymbol{\Psi}_{j}\left(\tilde{\lambda}_{j}(\boldsymbol{\theta}), \boldsymbol{\theta}\right)$ for DETD requires $2 p+2$ determinant evaluations. The computational complexity to evaluate a $N$-order determinant using the LU decomposition is $\mathcal{O}\left(N^{3}\right)$ [89], therefore the complexity of computing one evaluation of $\boldsymbol{\Psi}_{j}$ is $\mathcal{O}\left(2(p+1) N^{3}\right)$. 
On the contrary, EIGD requires to solve an $N$-order linear eigenvalue problem for each point $\boldsymbol{\theta}$. This type of problem also has a complexity of $\mathcal{O}\left(N^{3}\right)$, [89]. When the eigenvector $\tilde{\mathbf{q}}_{j}$ is found, the rest of operations are matrix multiplications. Thus, in the numerator there are $p$ matrix products while in the denominator there is only one. Each numerator product from Eq. (5.56) has the form $\mathbf{v}^{T} \mathbf{A v}$ where $\mathbf{v}$, A have sizes $N \times 1, N \times N$, respectively. Hence, to evaluate $\boldsymbol{\Psi}_{j}$ has a complexity of $\mathcal{O}\left(N^{3}+2(p+1) N^{2}\right)$.

For systems of relatively large size, the computation times are of the form $2 a(p+$ 1) $N^{3}$ and $b N^{3}$ for DETD and EIGD methods respectively, for certain $a, b$ positives. It is expected the EIGD method becomes faster than DETD method provided that $a \approx b$. Although the latter equality has not been demonstrated in this paper, a comparison of computation times has been included in the numerical examples in order to verify the better efficiency of EIGD than DETD.

To illustrate the efficiency of the developed method, three numerical examples will be analyzed in the following section for discrete (proportional and non-proportional) and continuous (non-proportional) structures.

\subsection{Numerical Examples}

\subsubsection{Example 1. Discrete structures with proportional damping}

In order to validate the proposed method in structures with proportional damping, a mass-lumped dynamical system with $N=6$ dof is analyzed. Fig. 5.1 shows the structural configuration. Each mass $m=10^{3} \mathrm{~kg}$ is attached to the next with a linear spring $k=10^{5} \mathrm{~N} / \mathrm{m}$ together with a non-viscous damper characterized by the kernel function $\mathcal{G}(t)$. A Biot's model with two exponentials is used to model the damper non-viscous behavior. Thus,

$$
\mathcal{G}(t)=\frac{c_{v}}{2}\left(\mu_{1} e^{-\mu_{1} t}+\mu_{2} e^{-\mu_{2} t}\right)
$$

where $c_{v}$ is the damping coefficient of the limit viscous model obtained when $\mu_{1}, \mu_{2} \rightarrow \infty$. The damping ratio $\zeta=c_{v} / 2 m \omega_{0}$ is commonly used instead of $c_{v}$, where $\omega_{0}=\sqrt{k / m}$ is a reference frequency. The Laplace transform of $\mathcal{G}(t)$ results in the viscoelastic damping function

$$
G(s)=\frac{c_{v}}{2}\left(\frac{\mu_{1}}{s+\mu_{1}}+\frac{\mu_{2}}{s+\mu_{2}}\right)
$$




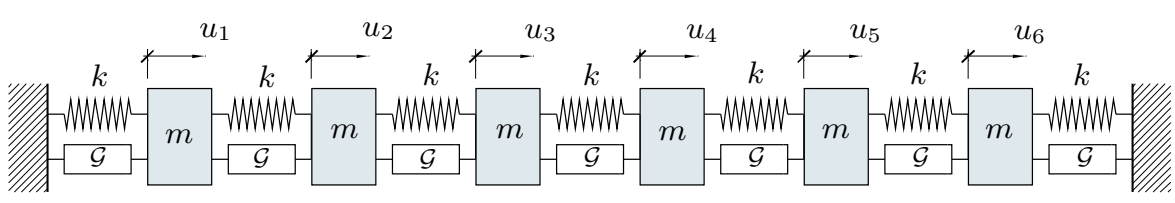

Figure 5.1: Example 1. Proportional Damping. Structural configuration with 6 discrete masses $m=10^{3} \mathrm{~kg}$ and stiffness $k=10^{5} \mathrm{~N} / \mathrm{m}$. Biot's damping model with two exponential kernels, $\mathcal{G}(t)=c_{v}\left(\mu_{1} e^{-\mu_{1} t}+\mu_{2} e^{-\mu_{2} t}\right) / 2$.

The mass matrix of the system is directly $\mathbf{M}=m \mathbf{I}_{6}$. The banded stiffness matrix is

$$
\mathbf{K}=k\left[\begin{array}{cccccc}
2 & -1 & 0 & 0 & 0 & 0 \\
-1 & 2 & -1 & 0 & 0 & 0 \\
0 & -1 & 2 & -1 & 0 & 0 \\
0 & 0 & -1 & 2 & -1 & 0 \\
0 & 0 & 0 & -1 & 2 & -1 \\
0 & 0 & 0 & 0 & -1 & 2
\end{array}\right]
$$

and, consequently, the damping matrix is $\mathbf{G}(s)=G(s) \mathbf{K} / k$. Since $\mathbf{G}(s)$ is proportional to the stiffness matrix, it becomes diagonal in the modal space. The elements of the matrix $\boldsymbol{\Gamma}(s)=\boldsymbol{\Phi}^{T} \mathbf{G}(s) \boldsymbol{\Phi}$ are

$$
\begin{aligned}
\Gamma_{j k}(s) & =\phi_{j}^{T} \mathbf{K} \phi_{k} \frac{G(s)}{k}=0, \quad j \neq k \\
\Gamma_{j j}(s) & =\phi_{j}^{T} \mathbf{K} \phi_{j} \frac{G(s)}{k}=\frac{\omega_{j}^{2} G(s)}{k}
\end{aligned}
$$

Using Eq. (5.8), the characteristic equation for the $j$ th mode is

$$
\mathcal{D}_{j}(s)=s^{2}+s \Gamma_{j j}(s)+\omega_{j}^{2}=0
$$

The damping is controlled by the three parameters $\left\{\zeta, \mu_{1}, \mu_{2}\right\}$. However, $\mu_{1}, \mu_{2}$ can be replaced by two non-dimensional parameters $\beta_{1}=\omega_{0} / \mu_{1}, \beta_{2}=\omega_{0} / \mu_{2}$. Then, the damping function adopts the form

$$
G(s)=\frac{c_{v}}{2}\left(\frac{\omega_{0}}{\beta_{1} s+\omega_{0}}+\frac{\omega_{0}}{\beta_{2} s+\omega_{0}}\right)
$$

When $\mu_{1}, \mu_{2} \rightarrow \infty$, the new parameters $\beta_{1}, \beta_{2} \rightarrow 0$ and the damping function degenerates in the limit to the viscous coefficient, $G(s) \rightarrow c_{v}=2 m \omega_{0} \zeta$; the characteristic equation of the $j$ th mode leads to the second order polynomial

$$
s^{2}+2 s \alpha_{j} \omega_{j} \zeta+\omega_{j}^{2}=0
$$



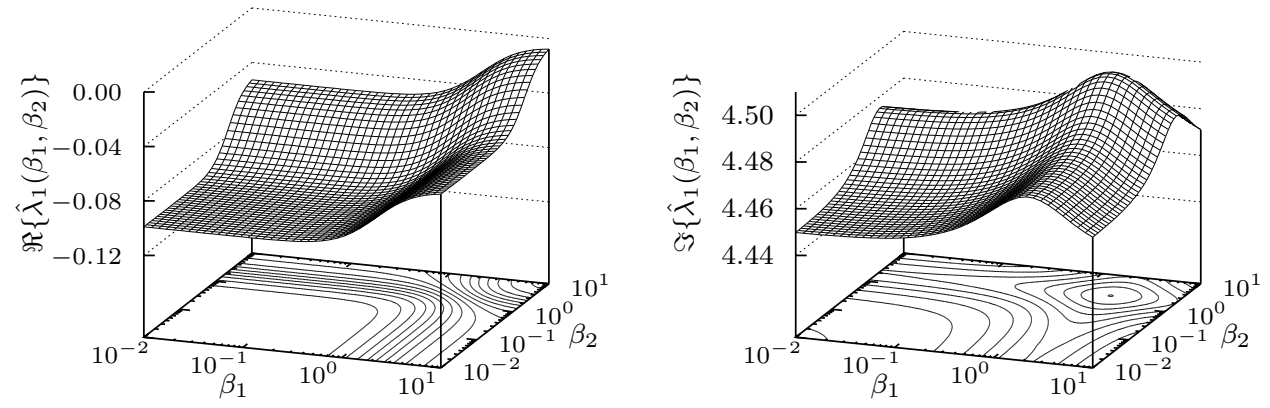

Figure 5.2: Example 1. Approximation of the 1st eigenvalue $\hat{\lambda}_{1}\left(\beta_{1}, \beta_{2}\right)$, as function of damping parameters $\beta_{1}=\omega_{0} / \mu_{1}, \beta_{2}=\omega_{0} / \mu_{2}, \zeta=0.05$. Real part (left) and imaginary part (right).

which solution is the pair of complex conjugate numbers

$$
\lambda_{j}^{0}=-\omega_{j} \alpha_{j} \zeta \pm i \omega_{j} \sqrt{1-\left(\alpha_{j} \zeta\right)^{2}}
$$

where $1 \leq j \leq N$ and $\alpha_{j}=\omega_{j} / \omega_{0}$. As a result, at the point $\left(\beta_{1}, \beta_{2}\right)=(0,0)$ a close solution of the $j$ th eigenvalue can be found as explicit function of $\zeta$. This allows to use the two-dimensional array $\boldsymbol{\theta}=\left(\beta_{1}, \beta_{2}\right)$ as a multi-variable parameter so that the eigenvalues may be the functions $\lambda_{j}=\lambda_{j}\left(\beta_{1}, \beta_{2}\right)$. The initial point $\boldsymbol{\theta}_{0}=(0,0)$ and its associated initial solution $\lambda_{j}^{0}$ have been calculated in Eq. (5.80). In order to apply the proposed method, it is necessary to compute the derivatives of the function $\Gamma_{j j}(s, \boldsymbol{\theta})$ evaluated in $(s, \boldsymbol{\theta})=\left(\lambda_{j}^{0}, \boldsymbol{\theta}_{0}\right)$

$$
\begin{aligned}
\Gamma_{j j}\left(\lambda_{j}^{0}, \boldsymbol{\theta}_{0}\right) & =\alpha_{j} \omega_{j} \zeta \\
\frac{\partial \Gamma_{j j}\left(\lambda_{j}^{0}, \boldsymbol{\theta}_{0}\right)}{\partial s} & =0 \\
\frac{\partial \Gamma_{j j}\left(\lambda_{j}^{0}, \boldsymbol{\theta}_{0}\right)}{\partial \beta_{1}} & =\frac{\partial \Gamma_{j j}\left(\lambda_{j}^{0}, \boldsymbol{\theta}_{0}\right)}{\partial \beta_{2}}=-\alpha_{j}^{2} \lambda_{j}^{0} \zeta
\end{aligned}
$$

Therefore, using Eq. (5.20) it can be verified that the components of $\nabla \lambda_{j}\left(\lambda_{j}^{0}, \boldsymbol{\theta}_{0}\right)$ are equal

$$
\frac{\partial \lambda_{j}\left(\lambda_{j}^{0}, \boldsymbol{\theta}_{0}\right)}{\partial \beta_{1}}=\frac{\partial \lambda_{j}\left(\lambda_{j}^{0}, \boldsymbol{\theta}_{0}\right)}{\partial \beta_{2}}=\frac{\alpha_{j}^{2} \zeta\left(\lambda_{j}^{0}\right)^{2}}{2 \lambda_{j}^{0}+\alpha_{j} \omega_{j} \zeta}
$$


Hence, the Taylor series expansion of $\lambda_{j}\left(\beta_{1}, \beta_{2}\right)$ around the point $\beta_{1}=\beta_{2}=0$ may be written explicitly as

$$
\tilde{\lambda}_{j}\left(\beta_{1}, \beta_{2}\right)=\lambda_{j}^{0}\left(1+\frac{\alpha_{j}^{2} \lambda_{j}^{0}\left(\beta_{1}+\beta_{2}\right)}{2 \lambda_{j}^{0}+\alpha_{j} \omega_{j} \zeta} \zeta\right)
$$

Essentially, the current approach is based on the estimation of the function $\boldsymbol{\Psi}_{j}(s, \boldsymbol{\theta})$ introduced in Eq. (5.20) through the particularized expression.

$$
\mathbf{\Psi}_{j}\left(\lambda_{j}, \beta_{1}, \beta_{2}\right) \approx \boldsymbol{\Psi}_{j}\left(\tilde{\lambda}_{j}\left(\beta_{1}, \beta_{2}\right), \beta_{1}, \beta_{2}\right)
$$

Using Eq. (5.29), the value of $\hat{\lambda}_{j}\left(\beta_{1}, \beta_{2}\right)$ can be computed, that represents an improved approximation with respect to that of the Taylor series expansion (5.83).

Fig 5.2 shows the real and imaginary part of the first eigenvalue as functions of $\left(\beta_{1}, \beta_{2}\right)$ in the range $0 \leq \beta_{1}, \beta_{2} \leq 10$. The form of the surface is essentially the same for the other modes due to the damping matrix proportionality. The real part monotonically increases with the parameters whereas the imaginary part always presents a maximum whose location varies with the mode. Notice that the real part is always negative, to ensure the decay of the response. Also, that for this eigenvalue, from Eq. (5.80) it can be appreciated that the components of $\lambda_{1}^{0}$ will be the intersection of the surfaces with the vertical axis.

To compare the eigenvalues from the proposed method and from the exact, the surfaces' contours are plotted for the modes $j=1,2,3$ in Fig. 5.3, continuous line for exact and discontinuous for our approach. The former are obtained solving Eq. (5.77) that degenerates into a fourth order polynomial, and the latter from the procedure in Section 5.3, Eq. (5.29). Real (left column) and imaginary (middle) values are superimposed, meanwhile the right column shows the error in percentage, as function of the parameters as

$$
\epsilon_{j}\left(\beta_{1}, \beta_{2}\right)=\frac{\left|\lambda_{j}\left(\beta_{1}, \beta_{2}\right)-\hat{\lambda}_{j}\left(\beta_{1}, \beta_{2}\right)\right|}{\left|\lambda_{j}\left(\beta_{1}, \beta_{2}\right)\right|}
$$

The left and middle contour plots show that the calculated surfaces obtained with the proposed method are very close to the exact ones, specially around $\beta_{1}=\beta_{2}=0$, where Eq. (5.72) predicted that the error order is $\mathcal{O}\left(h^{3}\right)$. This can clearly be observed in the error density plot: the smaller the parameters the lower the error; even far from the origin very good agreement between the curves is observed. 

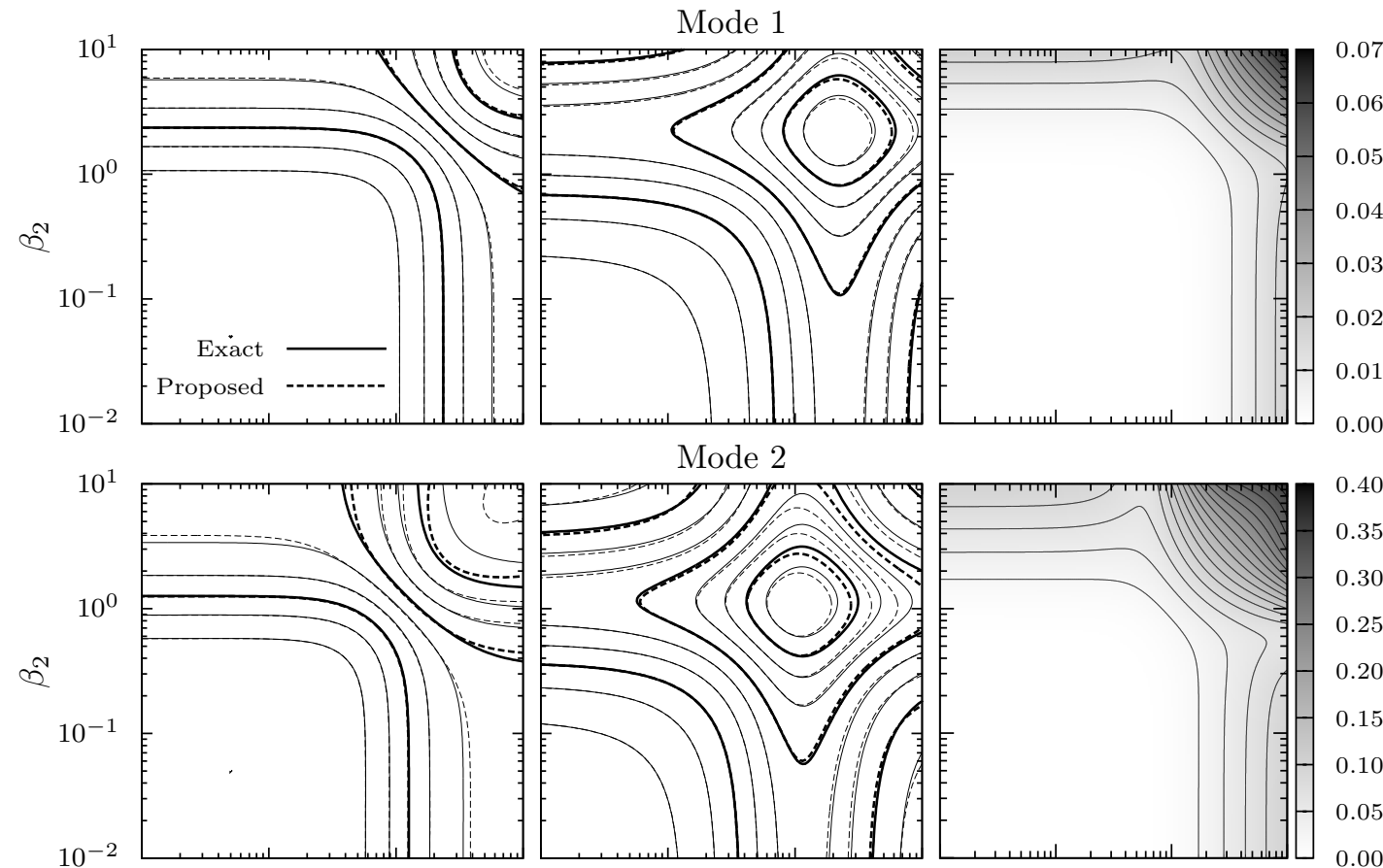

Mode 2
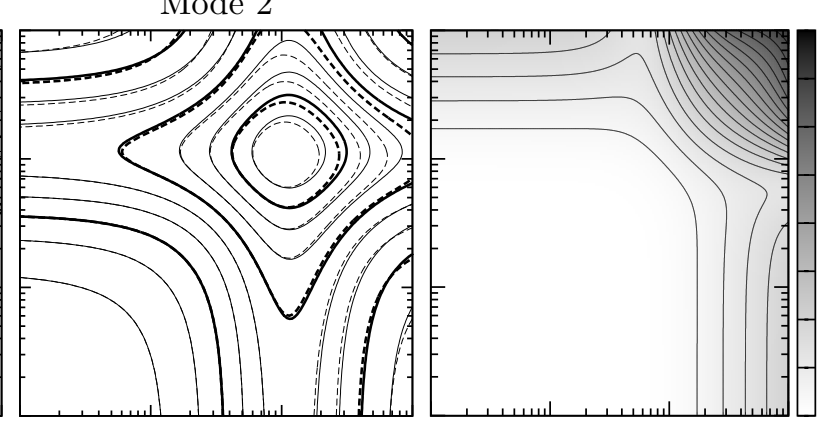

0.40 0.35 0.30 0.25 0.20 0.15 0.10 0.05 Mode 3
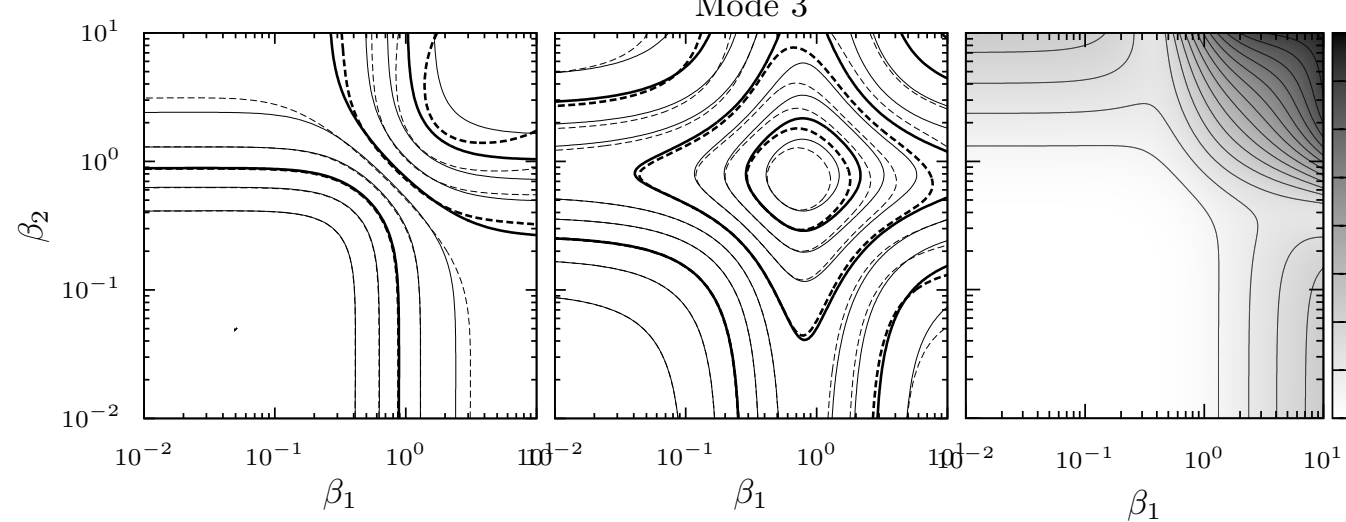

0.00

0.80 0.70 0.60 0.50 0.40 0.30 0.20 0.10 0.00

Figure 5.3: Example 1. Contours of eigenvalues, 1st, 2nd, 3rd modes for damping ratio $\zeta=0.05$. First column real part; second imaginary part; third relative error $(\%)$. 
It is also interesting to obtain a norm of the error for the considered range $\beta_{\text {min }} \leq$ $\beta_{1}, \beta_{2} \leq \beta_{\max }$ to compare the approximations for different modes. For that, the maximum error associated with the $j$ th mode can be introduced as

$$
\epsilon_{\max , j}=\max \left\{\epsilon_{j}\left(\beta_{1}, \beta_{2}\right): \beta_{\min } \leq \beta_{1}, \beta_{2} \leq \beta_{\max }\right\}
$$

Thus, the maximum error in the range of study is small for the first three modes: $\epsilon_{\max , 1}=0.07 \%, \epsilon_{\max , 2}=0.40 \%$ and $\epsilon_{\max , 3}=0.80 \%$, respectively.

The maximum error defined in Eq. (5.86) is a good indicator to compare the proposed and the Adhikari and Pascual's methods [31] (ADPA), with similar characteristics than the current: a) the computation of the eigenvalues is carried out by a non-iterative procedure and b) it is valid for proportionally damped structures. ADPA assumes that the $j$ th eigenvalue can be expressed as $s_{j}=s_{0 j}+\delta$, where $s_{0 j}$ is certain point adequately chosen and $\delta$ is a value to be obtained. The unknown $\delta$ is calculated expanding the characteristic equation $\mathcal{D}_{j}(s)$ from Eq. (5.9) around $s_{0 j}$ up to the second order. Then, solving the resulting second order polynomial

$$
\begin{aligned}
0 & =\mathcal{D}_{j}\left(s_{0 j}+\delta\right) \\
& \approx \mathcal{D}_{j}\left(s_{0 j}\right)+\frac{\partial \mathcal{D}_{j}\left(s_{0 j}\right)}{\partial s} \delta+\frac{\partial^{2} \mathcal{D}_{j}\left(s_{0 j}\right)}{\partial s^{2}} \frac{\delta^{2}}{2}
\end{aligned}
$$

The lowest of the two roots (in absolute value) is chosen. ADPA proposes as initial guess $s_{0 j}=-\xi_{j} \omega_{j}+i \omega_{j} \sqrt{1-\xi_{j}^{2}}$, where $\xi_{j}=\lim _{s \rightarrow 0} \Gamma_{j j}(s) / 2 \omega_{j}$, provided that this limit exists. Note that this method results in a closed-form expression funtion of the damping parameters although the correct root of Eq. (5.87) cannot be known a priori.

In Fig. 5.4(a), $\epsilon_{\max , j}$ has been represented for each mode using four values of the damping ratio, from $\zeta=0.01$ (lightly damped) to $\zeta=0.5$ (strongly damped). The results show that the accuracy of the proposed method depends directly on the level of damping: higher damping ratios worsen the approximation. Furthermore, for a fixed damping ratio the higher the mode the larger the error. The explanation of this fact can be found in the expression of the $j$ th mode damping function, $\Gamma_{j j}(s)=\omega_{j}^{2} G(s) / k$ from Eq. (5.76). Taking into account that the modes are ordered as $\omega_{j}<\omega_{j+1}$, it is clear that $\left|\Gamma_{j j}(s)\right|<\left|\Gamma_{j+1, j+1}(s)\right|$. This inequality can be interpreted as mode $j+1$ being more damped than mode $j$, that is, the quality of the results of the proposed method is expected to become poorer as shown in Fig. 5.4. Although the error for $\zeta=0.5$ is large, this level of damping is not usual in real structures that typically range in $\zeta<0.2$.

In Fig. 5.4(b), the maximum error for ADPA is shown. Note that the increasing trend of the error is again repeated due to the proportionality of the system. This 
method has proved to be more accurate than the proposed one for each mode because the difference between the exact and the estimated solution is of order $\mathcal{O}\left(\delta^{3}\right)$; this fact implies that the associated iterative scheme has cubic convergence. However, ADPA loses accuracy for non-proportional damping as will be shown in the following example.
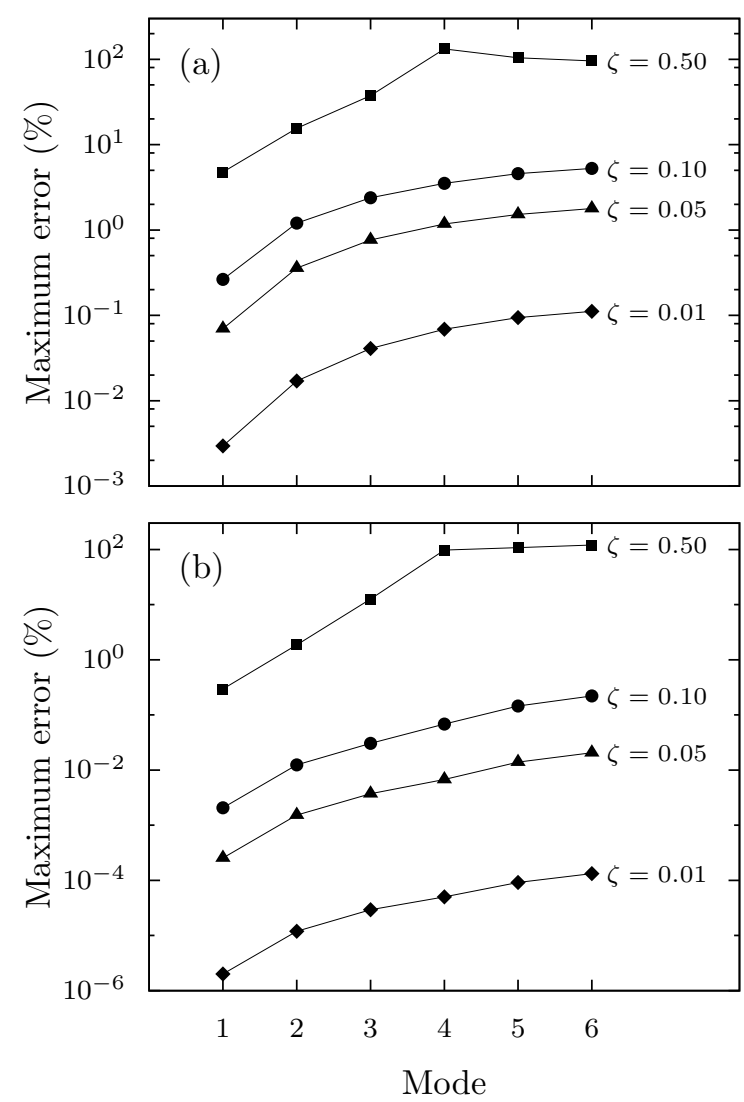

Figure 5.4: Example 1. Maximum error $\epsilon_{\max , j}(\zeta)$ for proportional damping systems through $0.01 \leq \beta_{1}, \beta_{2} \leq 10$. Proposed method (a), Adhikari-Pascual method (b). 


\subsubsection{Example 2. Discrete structures with non-proportional damping}

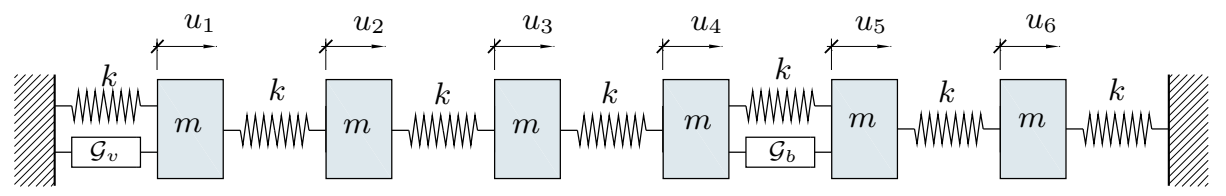

Figure 5.5: Example 2. Non-proportional Damping. Structural configuration with 6 discrete masses $m=10^{3} \mathrm{~kg}$, and stiffness $k=10^{5} \mathrm{~N} / \mathrm{m}$. Damping functions $\mathcal{G}_{v}(t)=$ $c_{1} \delta(t)$ (viscous) and $\mathcal{G}_{b}(t)=c_{2}\left(\nu e^{-\nu t}+\gamma e^{-\gamma t}\right) / 2$ (non-viscous). Parameters $\nu=5$, $\gamma=15 \mathrm{rad} / \mathrm{s}$.

In this example, the DETD and EIGD methods are compared and validated. For that end, the 6-dof lumped-mass discrete system represented in Fig. 5.5 is analyzed. The mass and linear spring values are $m=10^{3} \mathrm{~kg}, k=10^{5} \mathrm{~N} / \mathrm{m}$, as in Example 1. Two different dampers are located between the clamped end and the first mass and between masses 4, 5. The first is pure viscous, while the second is viscoelastic associated to a Biot's model with two exponential kernels. The damping functions for both dampers are

$$
\begin{aligned}
\mathcal{G}_{v}(t) & =c_{1} \delta(t) \\
\mathcal{G}_{b}(t) & =\frac{c_{2}}{2}\left(\nu e^{-\nu t}+\gamma e^{-\gamma t}\right)
\end{aligned}
$$

where $c_{1}, c_{2}$ are the damping coefficients; the second is obtained for $\nu, \gamma \rightarrow \infty$. The damping ratios are $\zeta_{1}=c_{1} / 2 m \omega_{0}, \zeta_{2}=c_{2} / 2 m \omega_{0}$, where $\omega_{0}=\sqrt{k / m}$ is a reference frequency. The Laplace transforms of the damping functions are

$$
\begin{aligned}
G_{v}(s) & =c_{1}=2 m \omega_{0} \zeta_{1} \\
G_{b}(s) & =m \omega_{0} \zeta_{2}\left(\frac{\nu}{s+\nu}+\frac{\gamma}{s+\gamma}\right)
\end{aligned}
$$

The mass and stiffness matrices are the same as those of Example 1, but the damping matrix $\mathbf{G}(s)$ depends on the location of the dampers. Thus,

$$
\mathbf{G}(s)=G_{v}(s) \mathbf{Q}_{1}+G_{b}(s) \mathbf{Q}_{2}
$$

where

$$
\mathbf{Q}_{1}=\left[\begin{array}{rrrrrr}
1 & 0 & 0 & 0 & 0 & 0 \\
0 & 0 & 0 & 0 & 0 & 0 \\
0 & 0 & 0 & 0 & 0 & 0 \\
0 & 0 & 0 & 0 & 0 & 0 \\
0 & 0 & 0 & 0 & 0 & 0 \\
0 & 0 & 0 & 0 & 0 & 0
\end{array}\right], \mathbf{Q}_{2}=\left[\begin{array}{rrrrrr}
0 & 0 & 0 & 0 & 0 & 0 \\
0 & 0 & 0 & 0 & 0 & 0 \\
0 & 0 & 0 & 0 & 0 & 0 \\
0 & 0 & 0 & 1 & -1 & 0 \\
0 & 0 & 0 & -1 & 1 & 0 \\
0 & 0 & 0 & 0 & 0 & 0
\end{array}\right]
$$


The damping model is governed by the parameters $\left\{\zeta_{1}, \zeta_{2}, \nu, \gamma\right\}$. When $\zeta_{1}=$ $\zeta_{2}=0$, the eigensolution can be directly computed because the system becomes undamped. Hence, it is reasonable to chose the parameters $\boldsymbol{\theta}=\left(\zeta_{1}, \zeta_{2}\right)$ so that the eigenvalues will be functions with form $\lambda_{j}\left(\zeta_{1}, \zeta_{2}\right)$. The application of the proposed method requires the evaluation of the function $\lambda_{j}\left(\zeta_{1}, \zeta_{2}\right)$ at the initial point $\boldsymbol{\theta}_{0}=(0,0)$ and the calculation of derivatives with respect to $\zeta_{1}$ and to $\zeta_{2}$. Therefore, the $j$ th eigenvector is $\mathbf{u}_{j}\left(\boldsymbol{\theta}_{0}\right)=\boldsymbol{\phi}_{j}$. From Eq. (5.38) and after some operations

$$
\begin{aligned}
\lambda_{j}(0,0) & =\lambda_{j}^{0}= \pm i \omega_{j} \\
\frac{\partial \lambda_{j}(0,0)}{\partial \zeta_{1}} & =-\rho_{j 1} \omega_{0} \\
\frac{\partial \lambda_{j}(0,0)}{\partial \zeta_{2}} & =-\rho_{j 2} \omega_{0} J\left(\lambda_{j}^{0}\right) \\
\mathbf{q}_{j}(0,0) & =(0, \ldots, 0, \stackrel{j}{1}, 0, \ldots, 0)^{T}
\end{aligned}
$$

where

$$
\begin{aligned}
\rho_{j 1} & =m^{2} \boldsymbol{\phi}_{j}^{T} \mathbf{Q}_{1} \boldsymbol{\phi}_{j} \\
\rho_{j 2} & =m^{2} \boldsymbol{\phi}_{j}^{T} \mathbf{Q}_{2} \boldsymbol{\phi}_{j} \\
J(s) & =\frac{1}{2}\left(\frac{\nu}{s+\nu}+\frac{\gamma}{s+\gamma}\right)
\end{aligned}
$$

The Taylor series expansion of $\lambda_{j}\left(\zeta_{1}, \zeta_{2}\right)$ around the point $\boldsymbol{\theta}_{0}=(0,0)$ is

$$
\tilde{\lambda}_{j}\left(\zeta_{1}, \zeta_{2}\right)=\lambda_{j}^{0}-\omega_{0}\left(\rho_{j 1} \zeta_{1}+J\left(\lambda_{j}^{0}\right) \rho_{j 2} \zeta_{2}\right)
$$

valid in a neighborhood of the initial values of the parameters $\zeta_{1}=\zeta_{2}=0$.

Figs. 5.6, 5.7 show the real (left) and imaginary (middle) part of the exact and of the EIGD approximated eigenvalues, in superimposed form. Both have been calculated for the range $0.01 \leq \zeta_{1}, \zeta_{2} \leq 1$. The first three modes (see Fig. 5.6) present a better fit in the contours, specially in the low range of the damping ratios (lightly damped systems). Furthermore, the accuracy is much more sensitive with respect to the parameter $\zeta_{1}$. In fact, the error may be considered almost negligible (less than $1 \%$ ) when $0 \leq \zeta_{1} \leq 0.6$ regardless of the $\zeta_{2}$ value. This characteristic can not be generalized to the rest of modes; indeed, for modes 4 th, 5 th, 6 th represented in Fig. 5.7, the error has a significant dependency on $\zeta_{2}$, specially for the 5th, 6th. For example, for the 6 th, it can be noticed that the results are almost independent of the first damping ratio $\zeta_{1}$. In general, the results plotted in Figs. 5.6, 5.7 validate EIGD for non-proportional systems and for lightly damped systems. In 


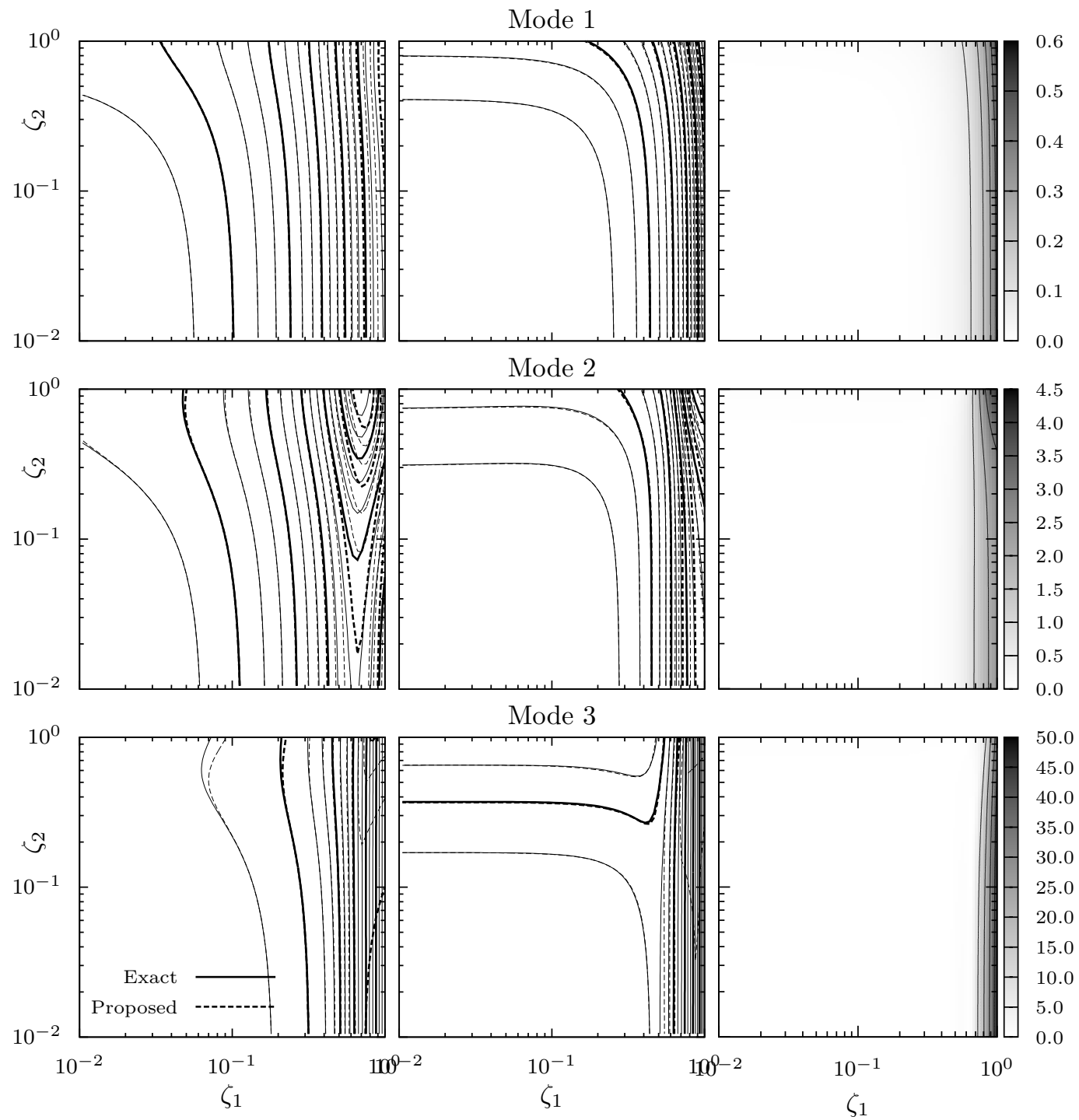

Figure 5.6: Example 2. Contours of eigenvalues, 1st, 2nd, 3rd modes; approximated solution by EIGD and relaxation parameters $\nu=5, \gamma=15 \mathrm{rad} / \mathrm{s}$. First column real part; second imaginary part; third relative error (\%). 


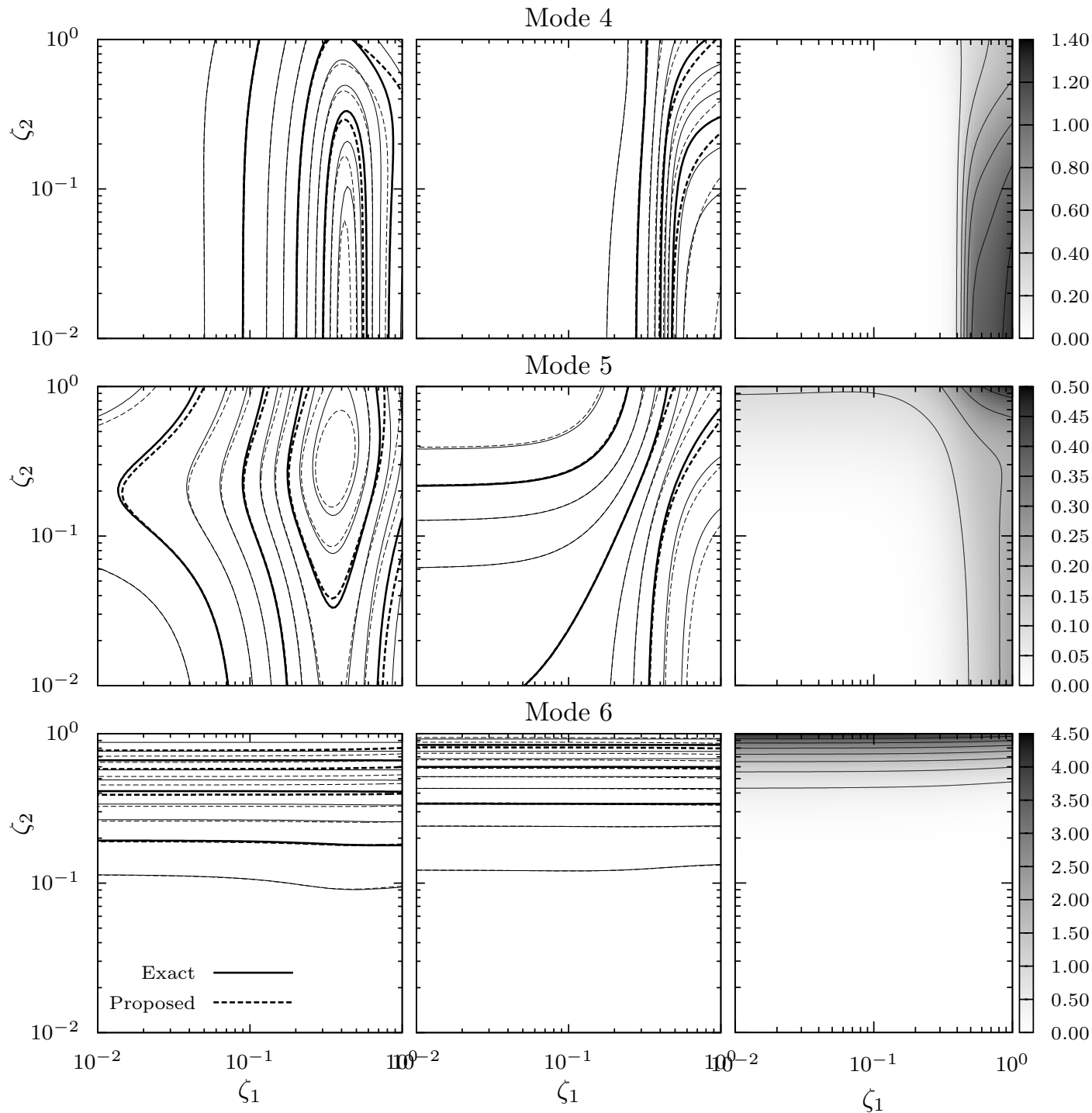

Figure 5.7: Example 2. Contours of eigenvalues, 1st, 2nd, 3rd modes; approximated solution by EIGD and elaxation parameters $\nu=5, \gamma=15 \mathrm{rad} / \mathrm{s}$. First column real part; second imaginary part; third relative error (\%). 
order to visualize the relationship between the error of the two proposed methods and the modes, the maximum error for each mode can be computed using the same procedure as before

$$
\epsilon_{\max , j}=\max \left\{\epsilon_{j}\left(\zeta_{1}, \zeta_{2}\right): \zeta_{\min } \leq \zeta_{1}, \zeta_{2} \leq \zeta_{\max }\right\}
$$

In Fig. 5.8, the values of $\epsilon_{\max , j}$ corresponding to EIGD and DETD are represented together with those obtained with ADPA. Since EIGD takes into account the nonproportionality, its estimation of eigenvalues is more accurate than both ADPA and DETD for all modes. In view of these results, it cannot be decided if DETD or ADPA is best since the former presents lower error for the 1st, 4th and 5th modes and the latter for the rest.

From a computational point of view, EIGD seems a priori to be more efficient than DETD as shown in Section 5.5.2. In order to validate this affirmation, Table 5.1 lists the computation times for both methods. In the last column it can be observed that EIGD is 1.5 to 2 times faster than DETD for this example. It follows that the application of DETD should be kept to small systems for which the analytical expression of the determinant $\mathcal{D}(s, \boldsymbol{\theta})$ is known. Only in this situation both methods may be competitive.

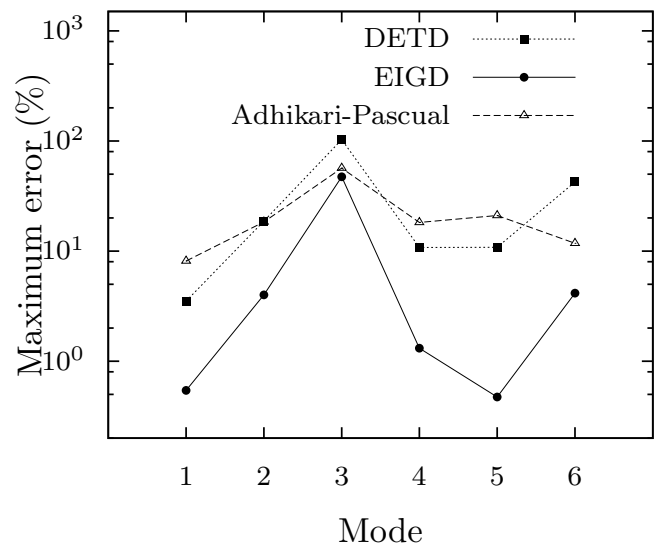

Figure 5.8: Example 2. Maximum error $\epsilon_{\max , j}$ for non-proportional damping systems calculated in the range $0.01 \leq \zeta_{1}, \zeta_{2} \leq 1$. 


\begin{tabular}{rrrr} 
Mode \# & EIGD & DETD & Ratio \\
\hline 1 & 1.705 & 2.773 & 0.61 \\
2 & 2.018 & 3.007 & 0.67 \\
3 & 2.819 & 5.715 & 0.49 \\
4 & 2.274 & 3.452 & 0.66 \\
5 & 2.012 & 3.250 & 0.62 \\
6 & 2.290 & 4.029 & 0.57 \\
\hline
\end{tabular}

Table 5.1: Comparison between computation times (in seconds) for the EIGD and DETD methods; ratio between them.

\subsubsection{Example 3. Continuous structures with non-proportional damping}

In this example, the proposed method is applied to a continuous steel beam with Young modulus $E=200 \mathrm{GPa}$ and density $\rho_{s}=7.85 \mathrm{t} / \mathrm{m}^{3}$. The cross section is rectangular with dimensions $20 \times 2 \mathrm{~mm}^{2}$ (width $\times$ height) and the length $600 \mathrm{~mm}$. Fig. 5.9 shows the setup and boundary conditions. The damping is introduced through two mechanisms: (i) two distributed unconstrained damping layers and (ii) a discrete viscoelastic damper located at the right end.

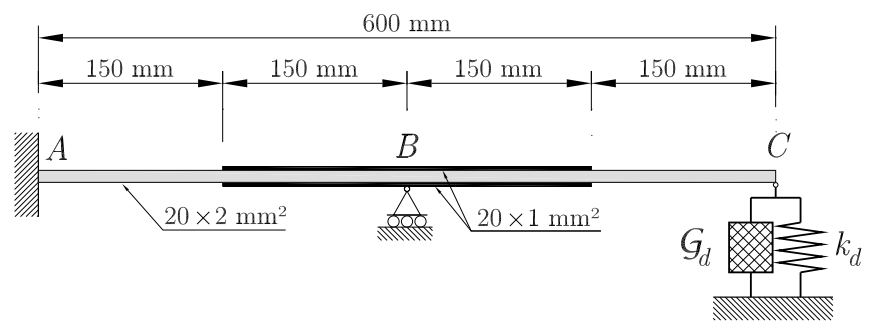

Figure 5.9: Example 3. Continuos beam with two free viscoelastic layers and a viscoelastic damper. Dimensions in $\mathrm{mm}$.

The two damping layers are $1 \mathrm{~mm}$ thick and perfectly bonded on the top and bottom surfaces at the central region as shown in Fig. 5.9. Since damping is induced from tension-compression in the layers, the constitutive equations relates longitudinal strain with stresses as

$$
\sigma_{x}(t)=E_{0} \epsilon_{x}(t)+\int_{-\infty}^{t} \mathcal{G}_{v}(t-\tau) \dot{\epsilon}_{x}(\tau) \mathrm{d} \tau
$$


Taking the Laplace transform of the previous expression the integro-differential relations are transformed into the algebraic expressions

$$
\hat{\sigma}_{x}(s)=\left[E_{0}+s G_{v}(s)\right] \hat{\epsilon}_{x}(s) \equiv \hat{E}_{v}(s) \hat{\epsilon}_{x}(s)
$$

where $s=i \omega$ is the Laplace eigenparameter and $\hat{E}_{v}(s)$ is the complex Young modulus. $\mathcal{G}_{v}(t)$ and $G_{v}(s)$ are the damping functions in time- and frequencydomain, respectively. For this example the damping material named 3M-ISD-112 (described in [90]) is chosen. This material has static Young modulus $E_{0}=204$ $\mathrm{kPa}$ and density $\rho_{v}=0.97 \mathrm{t} / \mathrm{m}^{3}$. The assumed damping function is a Biot's multiexponential model formed by $N_{e}=8$ hereditary kernels, resulting

$$
\mathcal{G}_{v}(t)=\frac{E_{0}}{1-g} \sum_{n=1}^{N_{e}} g_{n} e^{-t / \tau_{n}}
$$

where $g=\sum_{n=1}^{N} g_{n}$. Hence, the complex modulus can be written as $\hat{E}_{v}(s)=$ $E_{0}\left[1+s \varphi_{v}(s)\right]$, where the function $\varphi_{v}(s)=G_{v}(s) / E_{0}$ is given by

$$
\varphi_{v}(s)=\frac{1}{1-g} \sum_{n=1}^{N_{e}} \frac{g_{n} \tau_{n}}{1+\tau_{n} s}
$$

The parameters $E_{0}$ and $g_{n}, \tau_{n}$, for $1 \leq n \leq 8$ have been obtain by fitting the model and experimental data, and are shown in Table 5.2. More details on this damping material and on the fitting methodology can be found in [90, 91].

The vertical force in the viscoelastic damper $R_{C}$ and the associated displacement $v_{C}$ are related by the following fractional differential equation

$$
R_{C}+T_{r}^{\alpha} \frac{\mathrm{d}^{\alpha} R_{C}}{\mathrm{~d} t^{\alpha}}=k_{d} v_{C}+k_{\infty} T_{r}^{\alpha} \frac{\mathrm{d}^{\alpha} v_{C}}{\mathrm{~d} t^{\alpha}}
$$

The parameter $\alpha$ with $0<\alpha<1$ is the fractional exponent, $T_{r}>0$ is the relaxation time and $k_{\infty}>k_{d}$ is the storage rigidity. This rigidity is commonly replaced by the dimensionless storage coefficient $c=k_{\infty} / k_{d}>1$. The complex rigidity $\hat{K}_{d}(s)=\hat{R}_{C}(s) / \hat{v}_{C}(s)$ relates the above magnitudes in the Laplace domain. Taking the Laplace transform of Eq. (5.99), straight operations lead to

$$
\hat{K}_{d}(s)=k_{d} \frac{1+c\left(T_{r} s\right)^{\alpha}}{1+\left(T_{r} s\right)^{\alpha}} \equiv k_{d}\left[1+s \varphi_{d}(s)\right]
$$

where

$$
\varphi_{d}(s)=\frac{c-1}{s} \frac{\left(T_{r} s\right)^{\alpha}}{1+\left(T_{r} s\right)^{\alpha}}
$$

For the current example, the values $k_{d}=1.5 \mathrm{kN} / \mathrm{m}, \alpha=0.70, c=10^{3}$, and $T_{r}=5 \times 10^{-7} \mathrm{~s}$. have been chosen. For this combination, these values produce 


\begin{tabular}{rrr}
$n$ & $g_{n}$ & $\tau_{n}(\mathrm{~s})$ \\
\hline 1 & $3.1030 \mathrm{E}-03$ & $6.0730 \mathrm{E}-01$ \\
2 & $6.9000 \mathrm{E}-03$ & $1.5110 \mathrm{E}-01$ \\
3 & $1.2310 \mathrm{E}-02$ & $3.0380 \mathrm{E}-02$ \\
4 & $3.5340 \mathrm{E}-02$ & $8.1720 \mathrm{E}-03$ \\
5 & $6.5620 \mathrm{E}-02$ & $1.8210 \mathrm{E}-03$ \\
6 & $1.5410 \mathrm{E}-01$ & $4.8100 \mathrm{E}-04$ \\
7 & $2.6120 \mathrm{E}-01$ & $1.2010 \mathrm{E}-04$ \\
8 & $4.5400 \mathrm{E}-01$ & $3.0140 \mathrm{E}-05$ \\
\hline
\end{tabular}

Table 5.2: Damping parameters of the Biot's model for viscoelastic layer defined in Eq. (5.97). Relaxation time $\tau_{n}$ in seconds, $g_{n}$ dimensionless coefficient.

a relatively high damping, derived from the calculation of the loss factor peak, $\eta_{m}=1.72$. More details on this damping model and on the relation between the loss factor peak and the parameters $\alpha, c, T_{r}$ are available in the works of Pritz [20-22].

The beam is discretized in 1D, two-node finite elements of length $30 \mathrm{~mm}$. The mass and stiffness matrices are obtained applying the usual assumptions for beams with unconstrained viscoelastic layers, studied by Oberst [92]. Hence, the motion equation in frequency domain can be written in the standard form as

$$
\left[s^{2} \mathbf{M}+s \mathbf{G}(s)+\mathbf{K}\right] \hat{\mathbf{u}}(s)=\hat{\mathbf{F}}(s)
$$

where $\mathbf{M}=\mathbf{M}_{s}+\mathbf{M}_{v}$ is the mass matrix, $\mathbf{K}=\mathbf{K}_{s}+\mathbf{K}_{v}+\mathbf{K}_{d}$ the elastic stiffness matrix and $\mathbf{G}(s)=\varphi_{v}(s) \mathbf{K}_{v}+\varphi_{d}(s) \mathbf{K}_{d}$ the damping matrix. In these expressions the subscript $s$ is referred to the steel part, $v$ to the viscoelastic layers and $d$ to the discrete damper at section $C$.

In order to compute the eigenvalues with the proposed EIGD method, a multiparametric array $\boldsymbol{\theta}$ is required. For the current example, the vector $\boldsymbol{\theta}=\left(\tau_{1}, \ldots, \tau_{8}, c\right)$ with the initial value $\boldsymbol{\theta}_{0}=(0, \ldots, 0,1)$ is chosen. Note that the viscoelastic non linear eigenproblem evaluated at $\boldsymbol{\theta}_{0}$ is transformed into the linear undamped problem. This transformation allows the calculation of the initial solution, i.e. natural frequencies $\omega_{j}$ and real eigenvectors $\phi_{j}$, with relatively low computational effort. The eigenvalues are also obtained from the ADPA. However, as can easily be verified $\lim _{s \rightarrow 0} \Gamma_{j j}(s) / 2 \omega_{j}=\infty$, therefore since the proposed method starts at the undamped problem, it seems logical to choose $s_{0 j}=i \omega_{j}$ as the initial point to evaluate Eq. (5.86).

As before, the exact complex eigenvalues must be calculated to check the accuracy of the proposed and the ADPA methods. For that, the iterative approach of 


\begin{tabular}{rrrrrr} 
& & \multicolumn{2}{r}{ Proposed EIGD } & \multicolumn{2}{c}{ Adhikari-Pascual [31] } \\
\cline { 3 - 6 } Mode & Exact & Eigenvalue & Error (\%) & Eigenvalue & Error (\%) \\
\cline { 3 - 6 } & $-54.99+303.10 i$ & $-48.10+322.38 i$ & 6.644 & $-134.08+221.27 i$ & 36.946 \\
2 & $-45.27+595.16 i$ & $-43.95+604.53 i$ & 1.585 & $-101.27+550.03 i$ & 12.051 \\
3 & $-210.12+764.26 i$ & $-203.98+726.63 i$ & 4.810 & $-116.59+767.28 i$ & 11.806 \\
4 & $-68.78+1,619.07 i$ & $-69.73+1,618.22 i$ & 0.079 & $-63.43+1,616.56 i$ & 0.364 \\
5 & $-82.47+1,960.61 i$ & $-82.28+1,959.05 i$ & 0.080 & $-63.47+1,979.69 i$ & 1.372 \\
6 & $-51.51+3,299.74 i$ & $-51.70+3,299.59 i$ & 0.007 & $-48.48+3,300.88 i$ & 0.098 \\
7 & $-55.32+3,822.46 i$ & $-55.39+3,822.25 i$ & 0.006 & $-46.87+3,830.06 i$ & 0.297 \\
8 & $-43.09+5,647.99 i$ & $-43.17+5,648.01 i$ & 0.002 & $-41.49+5,648.74 i$ & 0.031 \\
9 & $-45.91+6,319.85 i$ & $-45.98+6,319.87 i$ & 0.001 & $-40.76+6,324.06 i$ & 0.105 \\
10 & $-37.54+8,594.64 i$ & $-37.55+8,594.78 i$ & 0.002 & $-36.37+8,595.31 i$ & 0.016 \\
11 & $-38.47+9,446.52 i$ & $-38.48+9,446.66 i$ & 0.001 & $-35.20+9,449.02 i$ & 0.044 \\
12 & $-34.47+12,253.32 i$ & $-34.50+12,253.50 i$ & 0.001 & $-33.69+12,253.76 i$ & 0.007 \\
13 & $-35.33+13,256.88 i$ & $-35.37+13,257.07 i$ & 0.002 & $-32.92+13,258.62 i$ & 0.022 \\
14 & $-32.14+16,543.38 i$ & $-32.09+16,543.65 i$ & 0.002 & $-31.48+16,543.77 i$ & 0.005 \\
15 & $-31.68+17,741.02 i$ & $-31.65+17,741.29 i$ & 0.002 & $-29.92+17,742.24 i$ & 0.012 \\
\hline
\end{tabular}

Table 5.3: Eigenvalues for example 3. Exact, proposed (EIGD) and Adhikari-Pascual methods with relative errors.

Ruhe [93] based on the generalization of Newton's method for nonlinear eigenvalue problems is used. The key idea is to transform the general problem of Eq. (5.3) into a linear one by expansion of the dynamic stiffness matrix $\mathbf{D}(s)$ around an initial guess, say $s_{0 j}$. The value $s_{0 j}+\delta$ is assumed to be very close to the $j$ th eigenvalue and hence, $\mathbf{D}\left(s_{0 j}+\delta\right) \mathbf{u}_{j} \approx \mathbf{0}$. Expanding this matrix up to the first order in terms of the unknown $\delta$ results in

$$
\left[\mathbf{D}\left(s_{0 j}\right)+\delta \frac{\partial \mathbf{D}\left(s_{0 j}\right)}{\partial s}\right] \mathbf{u}_{j} \approx \mathbf{0}
$$

This is a linear eigenvalue problem in $\delta$ that does not have to be solved entirely, since only the minimum (in absolute value) eigenvalue $\delta_{j}^{(0)}$ needs to be found; the next iteration is calculated as $s_{j}^{(1)}=s_{0 j}+\delta_{j}^{(0)}$. The iterative process consist on building the sequence $\left\{s_{j}^{(n)}\right\}$, which is locally convergent with quadratic speed. The initial guess is taken as the undamped eigenvalue $s_{0 j}=i \omega_{j}$ and shall be terminated when the relative error from one iteration to the next is less than $10^{-10}$. The solution thus obtained is considered exact.

In Table 5.3, the complex eigenvalues and the associated relative errors for EIGD and ADPA are listed for the first 15 modes. In view of the results, the eigenvalues calculated from EIGD present good agreement with the exact, specially for modes 4th and subsequents with error lower than $0.1 \%$. In addition, for the 15 modes shown, the EIGD error is lower than that of ADPA. This improvement is due to the non-proportionality of the damping matrix, that comes from the non-regular distribution of the viscoelastic layers together with the presence of the viscoelastic damper. The errors of both methods seems to have a monotonically decreasing trend and a question arises: Does exist a mode for which the ADPA method is more 
accurate than EIGD?. To answer this question, the relative error has been plotted in Fig. 5.10 extending the modal range up to mode 40th. For the first modes the trend reflected in the figure is the same as that observed at Table 5.3, however the error of ADPA seems to decrease faster than EIGD. In addition, the latter presents strong error decreases at modes 18th, 25th, 31th, 35th and 39th in disagreement with the observed trend. By inspection of the modal shapes, it is verified that these modes are precisely the extensional modes, while the rest are the flexural ones. Since the dof associated to the damper at $C$ only affects the flexural behavior, the extensional problem consequently presents lighter non-proportionality. This fact obviously improves the ADPA approximation at extensional modes.

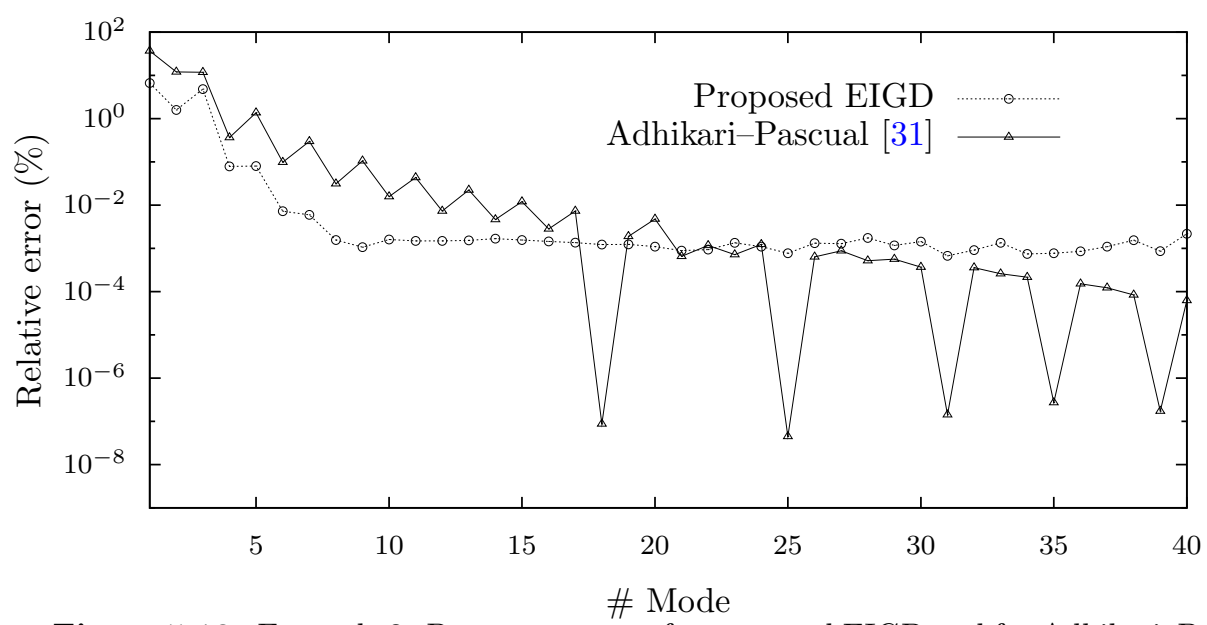

Figure 5.10: Example 3. Percentage error for proposed EIGD and for Adhikari-Pascual methods.

Considering now only flexural modes, the EIGD error is lower than that of ADPA for the first 21 modes and between the 21 th and 24 th both methods are similar. For 26th and subsequents the ADPA error follows a decreasing trend while EIGD error is constant, approximately equal to $0.001 \%$. Since the dof associated to the damper (principal cause of non-proportionality) becomes less active for higher modes, the damping matrix tends to be diagonally dominant with the mode number; this is the reason for which ADPA's error slightly improves for these almost proportional modes. 


\subsection{Conclusions}

The nonlinear eigenvalue problem in linear viscoelastic structures becomes of special importance for the time- and the frequency-domain solution of dynamic problems. In the present work, a new computational method to solve this eigenvalue problem in multi degree-of-freedom (dof) systems is proposed. The central idea is to consider the eigenvalues as function of certain multi-dimensional array $\boldsymbol{\theta}$, formed by damping parameters contained in the non-viscous damping function $\mathbf{G}(s, \boldsymbol{\theta})$. From these functions, the eigenvalues can be expanded in their Taylor series. The proposed method transforms the eigenrelations into ordinary differential equations with separate variables, so that the resolution is reduced to numerical integration, for which, quadrature methods can be used.

The technique is described for multi-dof systems, differentiating two cases: 1) proportional damping and 2) non-proportional damping. In the second, two variants of the method are proposed; the first, named DETD is based on the study of the determinant of the dynamic stiffness matrix and the second, named EIGD, uses the complex eigenvectors of the dynamic stiffness matrix. In order to study the numerical properties, the approximation order of the proposed solution has been evaluated. It is shown that this order is $\mathcal{O}\left(h^{3}\right)$, greater than the $\mathcal{O}\left(h^{2}\right)$ of the Taylor expansion. In addition, the computational complexity of the method is studied for non-proportional damping systems.

Three numerical examples are used to illustrate and validate the theoretical results. Proportional and non-proportional damping discrete systems are analyzed separately for a six-dof system. The results are compared with those of the noniterative method of Adhikari and Pascual (ADPA), valid only for proportional or lightly non-proportional damping. As expected from the error analysis, good agreements between the approximated and the exact solution are obtained, specially around the chosen initial point. For proportional damping systems the higher the mode the greater the error. For non-proportional, a comparative analysis between EIGD, DETD and ADPA has been carried out; for any mode, the first presents better accuracy than the others due to the special eigenvector approximation.

Finally, a continuous beam with non-proportional damping is analyzed. In this example, EIGD presents better accuracy than ADPA for modes with high nonproportionality. Further research is currently incorporating new numerical techniques oriented to avoid the numerical quadrature required for the eigenvalue estimation. 



\section{Bibliography}

[14] D.F. Golla and P.C. Hughes. "Dynamics of Viscoelastic Structures - A Time-domain, Finite-element Formulation." In: Journal of Applied MechanicsTransactions of the ASME 52.4 (1985), 897-906 (cit. on pp. 3, 20, 22, 24, $58,60,82,84,118,160,162,164,192)$.

[20] T. Pritz. "Analysis of four-parameter fractional derivative model of real solid materials." In: Journal of Sound and Vibration 195.1 (1996), 103-115 (cit. on pp. 4, 42, 45, 150, 192, 196, 206).

[21] T. Pritz. "Frequency dependences of complex moduli and complex Posson's ratio of real solid materials." In: Journal of Sound and Vibration 214.1 (1998), 83-104 (cit. on pp. 4, 45, 150, 206).

[22] T Pritz. "Loss factor peak of viscoelastic materials: Magnitude to width relations." In: Journal of Sound and Vibration 246.2 (2001), 265-280 (cit. on pp. 4, 45, 150, 196, 206).

[28] S. Adhikari. "Dynamics of Non-viscously Damped Linear Systems." In: Journal of Engineering Mechanics 128.3 (2002), 328-339 (cit. on pp. 4-6, $22,35,36,58,84,94,121,125,129,131,160-162,165,168,170,171,203)$.

[29] S. Adhikari. "Classical normal modes in non-viscously damped linear systems." In: AIAA Journal 39.5 (2001), pp. 978-980 (cit. on pp. 5, 35, 44, $63,84,121)$.

[30] J. Woodhouse. "Linear Damping Models For Structural Vibration." In: Journal of Sound and Vibration 215.3 (1998), 547-569 (cit. on pp. 5, 35, $58,121,170,171)$.

[31] Sondipon Adhikari and Blanca Pascual. "Eigenvalues of linear viscoelastic systems." In: Journal of Sound and Vibration 325.4-5 (2009), 1000-1011 (cit. on pp. 5, 21, 35, 41, 42, 69, 70, 76, 83, 119, 120, 141, 151, 152, 193).

[32] Sondipon Adhikari and Blanca Pascual. "Iterative Methods for Eigenvalues of Viscoelastic Systems." In: Journal of Vibration and Acoustics 133.2 (2011), pp. 021002.1-021002.7 (cit. on pp. 5, 21, 35, 42, 59, 83, 108, 119, $171,193)$. 
[33] M. Lázaro, J. L. Pérez-Aparicio, and M. Epstein. "Computation of eigenvalues in proportionally damped viscoelastic structures based on the fixedpoint iteration." In: Applied Mathematics and Computation 219.8 (2012), 3511-3529 (cit. on pp. 7, 59, 119, 193).

[40] D.J. McTavish and P.C. Hughes. "Modeling of Linear Viscoelastic Space Structures." In: Journal of Vibration and Acoustics-Transactions of the ASME 115.1 (1993), 103-110 (cit. on pp. 20, 24, 58, 82, 118, 160, 164).

[41] GA Lesieutre and DL Mingori. "Finite-Element Modeling of Frequencydependent Material Damping Using Augmenting Thermodynamic Fields." In: Journal of Guidance Control and Dynamics 13.6 (1990), 1040-1050 (cit. on pp. 20, 24, 58, 82, 119).

[46] Wei H. Yang. "A method for eigenvalues of sparse $\lambda$-matrices." In: International Journal for Numerical Methods in Engineering 19.6 (1983), pp. 943 948 (cit. on pp. 21, 83, 118, 193).

[47] KV Singh and YM Ram. "Transcendental eigenvalue problem and its applications." In: AIAA Journal 40.7 (2002), 1402-1407 (cit. on pp. 21, 83, 118).

[48] FW Williams and D Kennedy. "Reliable Use of Determinants to Solve Non-linear Structural Eigenvalue Problems Efficiently." In: International Journal for Numerical Methods in Engineering 26.8 (1988), 1825-1841 (cit. on pp. $21,83,118)$.

[49] E. M. Daya and M. Potier-Ferry. "A numerical method for nonlinear eigenvalue problems application to vibrations of viscoelastic structures." In: Computers 85 Structures 79.5 (2001), pp. 533 -541 (cit. on pp. 21, 83, 119).

[50] Laetitia Duigou, El Mostafa Daya, and Michel Potier-Ferry. "Iterative algorithms for non-linear eigenvalue problems. Application to vibrations of viscoelastic shells." In: Computer Methods in Applied Mechanics and Engineering 192.11-12 (2003), pp. 1323 -1335 (cit. on pp. 21, 119).

[51] H. Voss. "An Arnoldi Method for Nonlinear Eigenvalue Problems." In: BIT Numerical Mathematics 44 (2 2004), pp. 387-401 (cit. on pp. 21, 83, 118).

[52] H. Voss. "A Jacobi-Davidson method for nonlinear and nonsymmetric eigenproblems." In: Computers \& Structures 85.17-18 (2007), 1284-1292 (cit. on pp. 21, 83, 118).

[54] A Muravyov and SG Hutton. "Closed-form solutions and the eigenvalue problem for vibration of discrete viscoelastic systems." In: Journal of Applied Mechanics-Transactions of the ASME 64.3 (1997), 684-691 (cit. on pp. 21, 58, 83, 119, 160, 164, 193).

[55] A. Muravyov. "Forced vibration responses of viscoelastic structure." In: Journal of Sound and Vibration 218.5 (1998), 892-907 (cit. on pp. 21, 38, $83,119,160)$. 
[56] S. Menon and J. Tang. "A state-space approach for the dynamic analysis of viscoelastic systems." In: Computers \& Structures 82.15-16 (2004), pp. 1123 -1130 (cit. on pp. 21, 83, 119, 160, 164, 193).

[58] Sondipon Adhikari and Michael I. Friswell. "Eigenderivative analysis of asymmetric non-conservative systems." In: International Journal for $\mathrm{Nu}$ merical Methods in Engineering 51.6 (2001), pp. 709-733 (cit. on pp. 21, 119).

[59] S. Adhikari. "Derivative of Eigensolutions of Nonviscously Damped Linear Systems." In: AIAA Journal 40.10 (2002), 2061-2069 (cit. on pp. 21, 83, $119,126,132)$.

[60] F. Cortés and Maria Jesús Elejabarrieta. "Computational methods for complex eigenproblems in finite element analysis of structural systems with viscoelastic damping treatments." In: Computer Methods Appl. Mech. Engineering 195.44-47 (2006), 6448-6462 (cit. on pp. 21, 83, 119).

[61] F. Cortés and Maria Jesús Elejabarrieta. "An approximate numerical method for the complex eigenproblem in systems characterised by a structural damping matrix." In: Journal of Sound and Vibration 296.1-2 (2006), 166182 (cit. on pp. 21, 83, 119).

[64] P Muller. "Are the eigensolutions of a l-d.o.f. system with viscoelastic damping oscillatory or not?" In: Journal of Sound and Vibration 285.12 (2005), 501-509 (cit. on pp. 22, 38, 58, 84, 121, 162).

[71] S. Adhikari and J. Woodhouse. "Quantification of non-viscous damping in discrete linear systems." In: Journal of Sound and Vibration 260.3 (2003), pp. 499-518 (cit. on pp. 33, 88, 94, 125, 197, 203).

[74] S Adhikari. "Qualitative dynamic characteristics of a non-viscously damped oscillator." In: Proceedings of the Royal Society A-Mathematical Physical and Engineering Sciences 461.2059 (2005), 2269-2288 (cit. on pp. 38, 58, $84,121)$.

[79] RL Fox and MP Kapoor. "Rates of Change Eigenvalues and Eigenvectors." In: AIAA Journal 6.12 (1968), 2426-\& (cit. on pp. 83, 119).

[80] RB Nelson. "Simplified Calculation of Eigenvector Derivatives." In: AIAA Journal 14.9 (1976), 1201-1205 (cit. on pp. 83, 119).

[81] BP Wang. "Improved Approximate Methods for Computing Eigenvector Derivatives in Structural Dynamics." In: AIAA Journal 29.6 (1991), 10181020 (cit. on pp. 83, 119).

[82] OQ Zhang and A Zerva. "Iterative method for calculating derivatives of eigenvectors." In: AIAA Journal 34.5 (1996), 1088-1090 (cit. on pp. 83, 119). 
[83] DV Murthy and RT Haftka. "Derivatives of Eigenvalues and Eigenvectors of a General Complex Matrix." In: International Journal for Numerical Methods in Engineering 26.2 (1988), 293-311 (cit. on pp. 83, 119).

[84] DV Murthy and RT Haftka. "Approximations to Eigenvalues of Modified General Matrices." In: Computers \& Structures 29.5 (1988), 903-917 (cit. on pp. 83, 119).

[85] S. Adhikari. "Rates of change of eigenvalues and eigenvectors in damped dynamic system." In: AIAA Journal 37.11 (1999), 1452-1458 (cit. on pp. 83, 119).

[86] F. Cortés and Maria Jesús Elejabarrieta. "Finite element formulations for transient dynamic analysis in structural systems with viscoelastic treatments containing fractional derivative models." In: Computational Mechanics 69.10 (2007), 2173-2195 (cit. on p. 118).

[87] F. Cortés and Maria Jesús Elejabarrieta. "A direct integration formulation for exponentially damped structural systems." In: Computers \& Structures 87.5-6 (2009), 391-394 (cit. on p. 118).

[88] Lord Rayleigh. Theory of Sound. 2nd Edition. Dover Publications, 1945 (cit. on p. 119).

[89] J. H. Wilkinson. Algebraic Eigenvalue Problem. Oxford University Press, 1988 (cit. on pp. 135, 136).

[90] Marta L. Slanik et al. "Time Domain Finite Element Simulations of Damped Multilayered Beams Using a Prony Series Representation." In: Mechanics of Time-Dependent Materials 4 (3 2000), pp. 211-230 (cit. on p. 149).

[91] MIarie-Josée Potvin. "Comparison of Time-Domain Finite Element Modelling of Viscoelastic Structures Using an Efficient Fractional Voigt-Kelvin Model or Prony Series." PhD thesis. Faculty of Graduate Studies and Research. Department of Mechanical Engineering. McGill University, 2001 (cit. on p. 149).

[92] H. Oberst and K. Frankenfeld. "Über die Dämpfung der Biegeschwingungen Dünner Bleche Durch Fest Haftende Belage." In: J. Acustica 2 (1952), pp. 181-194 (cit. on pp. 150, 192, 194).

[93] A Ruhe. "Algorithms for Nonlinear Eigenvalue Problem." In: SIAM Journal on Numerical Analysis 10.4 (1973), 674-689 (cit. on pp. 151, 193, 207). 


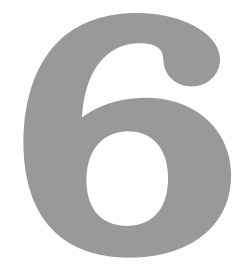

\section{An Equivalent Viscous Model for Linear Viscoelastic Systems}

\subsection{Introduction}

The simplest and most widespread mathematical representation of structural damping is the viscous model, whereby the dissipative forces are assumed to be proportional to the instantaneous velocities of the degrees of freedom (dof) of the system. Under the assumption of a small-deformation regime, the governing equations of the viscous model are of the following form

$$
\mathbf{M} \ddot{\mathbf{u}}+\mathbf{C} \dot{\mathbf{u}}+\mathbf{K u}=\mathbf{F}(t)
$$

where $\mathbf{M}, \mathbf{C}, \mathbf{K} \in \mathbb{R}^{N \times N}$ are, respectively, the constant mass, damping and stiffness matrices, and where $\mathbf{u}(t)$ and $\mathbf{F}(t)$ are the vectors of displacements and external forces, generally dependent on the time variable $t$. The exact or approximate solution of this system of linear simultaneous ODEs can be carried out by a variety of methods, including classical modal analysis. Modern multipurpose computer codes incorporate these methods and couple them, for example, with powerful finite-element techniques of structural analysis. 
However, in many applications, particularly in those pertaining to aerospace engineering, the simple viscous paradigm is inadequate. The main reason for this deficiency is the experimentally verified fact that the dissipative forces depend in an essential way on the history of the deformation, rather than just on the instantaneous velocity. While adhering to the linearity of the system, it is possible to incorporate the history dependence by means of a hereditary integral containing a kernel that depends on the backward-running time variable, as follows

$$
\mathbf{M} \ddot{\mathbf{u}}+\int_{-\infty}^{t} \mathcal{G}(t-\tau) \dot{\mathbf{u}} \mathrm{d} \tau+\mathbf{K u}=\mathbf{F}(t)
$$

where $\mathcal{G}(t) \in \mathbb{R}^{N \times N}$ is the matrix kernel. In practice, the kernel decays rapidly in time, which is a manifestation of the phenomenon of fading memory, according to which the events in the recent past are more significant in determining the present state than those in the distant past. Mathematically speaking, the damping forces in Eq. (6.2) have been expressed by means of a convolution integral. Somewhat imprecisely, we will refer to the hereditary paradigm as the viscoelastic model (VEM), reserving the name of viscous model (VM) for the particular case described by Eq. (6.1).

Several works have been focused on improving the viscoelastic models applied to mechanical systems; the works of Flugge [10], Nashif [11] and Jones [12] are of special relevance, containing the main contributions in this field. The non-viscous models are characterized by integro-differential equations and require new solution methods. In this sense, the vast majority of the methods aimed at solving Eq. (6.2) use techniques based on the state-space approach. Thus, Golla \& Hughes [14] and McTavish \& Hughes [40] introduced the GHM method using new internal variables. Muravyov [55, 94] and Muravyov and Hutton [54, 73] formulated a general Laplace domain-based method for isotropic and homogeneous hereditary materials with exponential kernels, analyzing the forced as well as the free vibration response. Menon and Tang [56] proposed a solution for viscoelastic problems with multi-exponential kernels based on the transformation of the characteristic equation into a certain polynomial. Hence, the order of the system was augmented as many times as the number of the exponential kernels that were used. A work of special importance in the last years concerning dynamics of non-symetric, nonviscous systems is due to Adhikari [28], in which analytical solutions for a general viscoelastic system were given as a function of the set of eigenvalues and eigenvectors of the system.

Due to the mathematical simplicity of viscously damped systems, it is a common practice to search equivalent dynamic models that are able to replicate the response of the original with enough accuracy. Bandstra [95] obtained for single dof systems the equivalent viscous damping for different nonlinear systems using a 
method based on the equivalence of energy dissipation. Related to linear problems applied to one-dimensional structures, Banks [96] used the modal analysis for the extraction of the set of modal damping ratios. These ratios were deduced for the special case of viscous-air damping combined with a Kelvin-Voigt constitutive relationship. In the particular case of complex structures with added viscoelastic dampers, different methods have been proposed for their modeling through equivalent viscous systems. Lima [97] suggested an efficient methodology for assembling structural systems with viscoelastic dampers based on a frequency response function coupling technique. Ungar \& Kerwin [98] proposed in 1962 the Modal Strain Energy method for obtaining the modal damping ratio in a general viscoelastic system. This method was implemented in a Finite Element Program by Johnson \& Kienholz [99]. Bilbao et al. [100] proposed a proportional damping matrix, using optimization based methods. Genta \& Amati [101] studied hysteretic models in rotordynamics and their equivalent damping ratios, analyzing the advantages and disadvantages of the ratios to obtain the time-domain response. For the specific case of a viscoelastic system based on fractional calculus, Makris [102] proposed an equivalent viscous model giving an equivalent damping ratio and a new natural frequency for viscoelastic dampers in single dof systems. The theoretical problem of finding a second order model equivalent to a general viscoelastic system has been studied by Segalman [103], assuming low viscoelasticity; an equivalent second-order model is deduced, defining a damping matrix and a new stiffness matrix. However, the resulting model is not viscous, due to the fact that the involved matrices are in general defined in the complex domain. Recently, Adhikari [104] has also proposed a second--order system in which the key idea is the evaluation of the viscoelastic function in each complex frequency, also assuming low viscoelasticity.

The published research about the construction of equivalent models for viscoelastic systems is, in general, oriented only to find an equivalent damping matrix. Modifications of the other dynamic matrices, say mass and stiffness, are usually not introduced. In addition, light viscoelasticity is an hypothesis commonly assumed in the majority of the cases.

In his landmark article, Adhikari [28] extended the classical modal analysis to treat the general VEM. In spite of the clarity of the analysis, the implementation of the fully fledged procedure in a numerical context remains a challenge in most practical applications, in which the number of degrees of freedom is very large. Building upon Adhikari's work [28], this paper proposes an approximate technique which, in some sense, is the 'best' approximation to a VEM by means of a VM, which we call an Equivalent Viscous Model (EVM). Some of the indications of how to build this model are provided by Adhikari himself. Indeed, he observes that "it appears that the number of nonviscous modes is not very high and also their contribution 
to the global dynamic response is not very significant." On the basis of this observation and of Adhikari's detailed calculations, we propose to construct the EVM by retaining precisely the complex eigenvalues of the dynamic problem (6.2) in the Laplace transform domain and discarding the non-viscous modes altogether. The mass, damping and stiffness matrices of the EVM are constructed on the basis of the normal modes of the undamped system and the complex eigenvalues of the full VEM. To assess the quality of the proposed technique, rigorous bounds are provided for the error of the approximation.

\subsection{Fundamentals of the Proposed Method}

\subsubsection{Eigenproblem in Viscoelastic Structures}

The eigenvalues and eigenvectors of a viscoelastic system have a special relevance in the construction of the proposed method. Their computation can be performed transforming the dynamical problem into a free motion one with $\mathbf{F}(t)=\mathbf{0}$ in Eq. (6.2). Taking now the Laplace transform, the following nonlinear eigenvalue problem is obtained

$$
\left[s^{2} \mathbf{M}+s \mathbf{G}(s)+\mathbf{K}\right] \hat{\mathbf{u}}(s)=\mathbf{0}
$$

where $\mathbf{G}(s), \hat{\mathbf{u}}(s)$ are, respectively, the Laplace transforms of $\mathcal{G}(t), \mathbf{u}(t)$. The eigenvalues are the roots the characteristic equation given by

$$
\operatorname{det}\left[s^{2} \mathbf{M}+s \mathbf{G}(s)+\mathbf{K}\right]=0
$$

Assuming that the damping matrix $\mathbf{G}(s)$ verifies the necessary conditions to define a dissipative motion [14], the number of eigenvalues will be $m \geq 2 N[28,64,73]$, and the complete set of roots can be represented as

$$
\left\{s_{1}, \cdots, s_{N}, s_{1}^{*}, \cdots, s_{N}^{*}, s_{2 N+1}, \cdots, s_{m}\right\}
$$

where the first $2 N$ eigenvalues are $N$ pairs of complex-conjugate eigenvalues with oscillatory nature and the last $m-2 N$ are named nonviscous roots, a set of real negative eigenvalues corresponding to non-oscillatory or overcritically damped modes. In general, the number of nonviscous roots will depend on the functional form of matrix $\mathbf{G}(s)$.

A priori, there is no reason to restrict the current method to symmetric systems. However, for the purposes of this paper it will be assumed that the matrices involved in the model are symmetric, that is, $\mathbf{M}=\mathbf{M}^{T}, \mathbf{K}=\mathbf{K}^{T}, \mathbf{G}(s)=\mathbf{G}^{T}(s)$, leaving for further works the asymmetric case. Hence, under the assumption of 
symmetry, it is not necessary to differentiate between left and right eigenvectors. Therefore, introducing the notation $\mathbf{u}_{j}$ for the $j$ th eigenvector, the following relationship can be written

$$
\mathbf{D}\left(s_{j}\right) \mathbf{u}_{j}=\mathbf{0}, \quad 1 \leq j \leq m
$$

$\mathbf{D}(s)$ being the dynamic stiffness matrix defined by

$$
\mathbf{D}(s) \equiv s^{2} \mathbf{M}+s \mathbf{G}(s)+\mathbf{K}
$$

In the set of eigenvalues of the VEM, those with oscillatory nature, corresponding with the $N$ pairs of complex conjugate numbers, play an important role in the construction of the proposed EVM. Furthermore, the latter has as a main feature to share its complex eigenvalues with the VEM.

\subsubsection{The Equivalent Viscous Model}

The free motion of the undamped system associated with the VEM (6.2) is governed by

$$
\mathbf{M u}+\mathbf{K u}=\mathbf{0}
$$

Let $\omega_{j}$ and $\phi_{j} \in \mathbb{R}^{N}$ be the $j$ th natural frequency and its eigenvector, respectively. The classical orthogonal eigenrelations may be written as

$$
\phi_{j}^{T} \mathbf{M} \phi_{k}=\delta_{j k}, \quad \phi_{j}^{T} \mathbf{K} \phi_{k}=\omega_{j}^{2} \delta_{j k}
$$

where $\delta_{j k}$ is the Kronecker delta. The system $\left\{\phi_{j}\right\}_{j=1}^{N}$ forms a base of the space $\mathbb{R}^{N}$. Let us consider now any $2 N$ positive real numbers $\left\{\zeta_{e j}, \omega_{e j}\right\}_{j=1}^{N}$, with $0<$ $\zeta_{e j}<1$ and $\omega_{e j}>0$. These numbers together with the vectors $\left\{\boldsymbol{\phi}_{j}\right\}_{j=1}^{N}$, always allow to construct a symmetric viscous model with proportional damping. For that, let us define the diagonal matrices

$$
\begin{aligned}
\mathbf{C}_{e}^{\prime} & =\operatorname{diag}\left[2 \zeta_{e j} \omega_{e j}\right] \\
\boldsymbol{\Lambda}_{e} & =\operatorname{diag}\left[\omega_{e j}^{2}\right]
\end{aligned}
$$

and the matrix $\boldsymbol{\Phi}=\left[\phi_{1}, \ldots, \phi_{N}\right]$. Then, under these conditions, the following matrices

$$
\begin{aligned}
\mathbf{M}_{e} & =\mathbf{M} \\
\mathbf{C}_{e} & =\boldsymbol{\Phi}^{-T} \mathbf{C}_{e}^{\prime} \boldsymbol{\Phi}^{-1} \\
\mathbf{K}_{e} & =\boldsymbol{\Phi}^{-T} \boldsymbol{\Lambda}_{e} \boldsymbol{\Phi}^{-1}
\end{aligned}
$$

are real, symmetric and become diagonal in the eigenbasis $\boldsymbol{\Phi}$. Furthermore, it can be directly verified that the second-order linear system of differential equations

$$
\mathbf{M}_{e} \ddot{\mathbf{u}}+\mathbf{C}_{e} \dot{\mathbf{u}}+\mathbf{K}_{e} \mathbf{u}=\mathbf{F}(t)
$$


has as eigenvalues the $N$ complex conjugate pairs

$$
\left\{-\omega_{e j} \zeta_{e j} \pm i \omega_{e j} \sqrt{1-\zeta_{e j}^{2}}\right\}_{j=1}^{N}
$$

where $i=\sqrt{-1}$ is the imaginary unity.

Let us assume that the set of complex eigenvalues of the VEM defined in Eq. (6.3), namely $\left\{s_{j}, s_{j}^{*}\right\}_{j=1}^{N}$, is known. In addition, let $\left\{\boldsymbol{\phi}_{j}\right\}_{j=1}^{N}$ be the system of undamped eigenvectors. We are interested to obtain the unknown $2 N$ numbers $\zeta_{e j}$ and $\omega_{e j}$, $1 \leq j \leq N$ such that the eigenvalues of the VEM be the same as those of the EVM. Therefore, the following $2 N$ equalities arise

$$
\begin{array}{ll}
s_{j}=-\omega_{e j} \zeta_{e j}+i \omega_{e j} \sqrt{1-\zeta_{e j}^{2}}, & 1 \leq j \leq N \\
s_{j}^{*}=-\omega_{e j} \zeta_{e j}-i \omega_{e j} \sqrt{1-\zeta_{e j}^{2}}, & 1 \leq j \leq N
\end{array}
$$

The values of $\zeta_{e j}$ and $\omega_{e j}$ may be solved as

$$
\zeta_{e j}=-\frac{s_{j}+s_{j}^{*}}{2 \sqrt{s_{j} s_{j}^{*}}}, \quad \omega_{e j}=\sqrt{s_{j} s_{j}^{*}}
$$

These parameters will be named respectively equivalent damping ratio and equivalent natural frequency of the $j$ th mode. Note that $\zeta_{e j}$ is well defined since the real part of any eigenvalue, say $\Re\left\{s_{j}\right\}=\left(s_{j}+s_{j}^{*}\right) / 2$, is negative, in order to characterize a dissipative motion. The dynamic matrices $\mathbf{M}_{e}, \mathbf{C}_{e}$ and $\mathbf{K}_{e}$ deduced from the values given in Eq. (6.16) constitute the proposed EVM.

Methods based on state-space approach are commonly used to solve linear viscoelastic systems. However, these methods require new additional internal variables [14, 40, 54, 56], which involves a high computational cost for systems with relatively large number of dof. Therefore, we propose the EVM as an efficient substitute of the original VEM because, as shown above, the former has the same complex eigenvalues as the latter, but the same eigenvectors as the undamped system. In consequence, it is expected that the response obtained with EVM can be considered as a good approximation, specially when the VEM is also proportional. This fact allows the use of standard tools focused on solving structural dynamics problems with viscous damping, implemented in the majority of finite element computational packages.

In order to provide the EVM with robust theoretical foundations, the norm of the difference between the Transfer Functions (TF) will be bounded in the following 
subsection. In addition, it will also be shown that the EVM previously defined may be deduced from an expansion of the exact TF.

\subsection{Decomposition of the Transfer Function}

\subsubsection{The Transfer Function}

Considering the steady-state motion equation in the Laplace domain, the relationship between the response, $\hat{\mathbf{u}}(s)$, and the external forces, $\hat{\mathbf{F}}(s)$, is given by

$$
\mathbf{D}(s) \hat{\mathbf{u}}(s)=\hat{\mathbf{F}}(s)
$$

From the previous equation, the response vector can be obtained by inversion of the dynamic stiffness matrix as

$$
\hat{\mathbf{u}}(s)=\mathbf{D}^{-1}(s) \hat{\mathbf{F}}(s) \equiv \mathbf{H}(s) \hat{\mathbf{F}}(s)
$$

where

$$
\mathbf{H}(s)=\left[s^{2} \mathbf{M}+s \mathbf{G}(s)+\mathbf{K}\right]^{-1} \in \mathbb{C}^{N \times N}
$$

is the TF. tHE Computation of one inverse matrix corresponding to each $s$ is not an efficient procedure to obtain the TF, specially for large systems. Hence, alternative methods based on the information given by the eigenvalue solution may be used. Adhikari [28] studied the dynamics of the viscoelastic systems and obtained closed solutions for the TF taking into account the total set of complex eigenvalues and eigenvectors. From the residue theorem, the TF of the original viscoelastic model (VEM) can be expressed as function of its poles and residues. Thus, using Adhikari's nomenclature

$$
\mathbf{H}(s)=\sum_{j=1}^{m} \frac{\mathbf{R}_{j}}{s-s_{j}}
$$

where

$$
\mathbf{R}_{j} \equiv \lim _{s \rightarrow s_{j}}\left(s-s_{j}\right) \mathbf{H}(s), \quad 1 \leq j \leq m
$$

is the residue of the $\mathrm{TF}$ at the pole $s_{j}$, for $1 \leq j \leq m$. Starting from the definition and using the linear eigenvalue problem of the dynamic stiffness matrix, Adhikari calculated the $j$ th residue, obtaining an expression as function exclusively of the VEM complex eigensolutions

$$
\mathbf{R}_{j}=\frac{\mathbf{u}_{j} \mathbf{u}_{j}^{T}}{\mathbf{u}_{j}^{T} \frac{\partial \mathbf{D}\left(s_{j}\right)}{\partial s} \mathbf{u}_{j}}, \quad 1 \leq j \leq m
$$


Introducing the results of the previous expression in Eq. (6.20) the TF may be expressed as

$$
\mathbf{H}(s)=\sum_{j=1}^{m} \frac{\mathbf{R}_{j}}{s-s_{j}}=\sum_{j=1}^{m} \frac{\gamma_{j} \mathbf{u}_{j} \mathbf{u}_{j}^{T}}{s-s_{j}}
$$

where the coefficient $\gamma_{j}$ is

$$
\gamma_{j}=\left[\mathbf{u}_{j}^{T} \frac{\partial \mathbf{D}\left(s_{j}\right)}{\partial s} \mathbf{u}_{j}\right]^{-1}, \quad 1 \leq j \leq m
$$

Such as was previously described in Section 6.2.1, among the $m$ eigenvalues, there exist $N$ pairs of complex conjugate values and $m-2 N$ nonviscous roots. In the previous $\mathrm{TF}$ expression, the effect of the different eigenvalues and their corresponding eigenvectors may be considered in separated form, resulting in

$$
\mathbf{H}(s)=\sum_{j=1}^{N}\left[\frac{\gamma_{j} \mathbf{u}_{j} \mathbf{u}_{j}^{T}}{s-s_{j}}+\frac{\gamma_{j}^{*} \mathbf{u}_{j}^{*} \mathbf{u}_{j}^{*^{T}}}{s-s_{j}^{*}}\right]+\sum_{j=2 N+1}^{m} \frac{\gamma_{j} \mathbf{u}_{j} \mathbf{u}_{j}^{T}}{s-s_{j}}
$$

Using the previous expression, Adhikari obtained the TF of undamped and viscously damped systems as particular cases. As it will be shown later, under certain conditions the TF of the VEM, given by Eq. (6.25), may be approximated by the TF of the EVM. For the purposes of the present work it is necessary to achieve a close expression of the latter. Again, since the complex eigenvalues of the EVM are the same as those of the VEM and the eigenvectors are $\left\{\phi_{1}, \ldots, \phi_{N}\right\}$, the TF of the EVM has the form

$$
\mathbf{H}_{e}(s)=\sum_{j=1}^{N}\left[\frac{\gamma_{e j} \phi_{j} \boldsymbol{\phi}_{j}^{T}}{s-s_{j}}+\frac{\gamma_{e j}^{*} \boldsymbol{\phi}_{j} \boldsymbol{\phi}_{j}^{T}}{s-s_{j}^{*}}\right]
$$

where

$$
\gamma_{e j}=\left[\boldsymbol{\phi}_{j}^{T} \frac{\partial \mathbf{D}_{e}\left(s_{j}\right)}{\partial s} \boldsymbol{\phi}_{j}\right]^{-1}, \quad 1 \leq j \leq N
$$

In order to obtain the value of $\gamma_{e j}$, let us introduce the dynamic stiffness matrix of the EVM

$$
\mathbf{D}_{e}(s)=s^{2} \mathbf{M}_{e}+s \mathbf{C}_{e}+\mathbf{K}_{e}
$$

From Eq. (6.12), pre-- and post-multiplying this equation by the vector $\phi_{j}$ and its transpose, the previous expression yields

$$
\boldsymbol{\phi}_{j}^{T} \mathbf{D}_{e}(s) \phi_{j}=s^{2}+2 \zeta_{e j} \omega_{e j} s+\omega_{e j}^{2}
$$

In the previous equation the values of $\zeta_{e j}$ and $\omega_{e j}$ can be substituted by theirs expressions obtained from Eq. (6.16), resulting in

$$
\phi_{j}^{T} \mathbf{D}_{e}(s) \phi_{j}=s^{2}-\left(s_{j}+s_{j}^{*}\right) s+s_{j} s_{j}^{*}=\left(s-s_{j}\right)\left(s-s_{j}^{*}\right)
$$


From its definition, the coefficient $\gamma_{e j}$ is

$$
\gamma_{e j}=\left[\boldsymbol{\phi}_{j}^{T} \frac{\partial \mathbf{D}_{e}\left(s_{j}\right)}{\partial s} \boldsymbol{\phi}_{j}\right]^{-1} \equiv \frac{1}{\rho_{e j}}, \quad 1 \leq j \leq N
$$

whence, using Eq. (6.30),

$$
\rho_{e j}=\left.\frac{\partial}{\partial s} \boldsymbol{\phi}_{j}^{T} \mathbf{D}_{e}(s) \boldsymbol{\phi}_{j}\right|_{s=s_{j}}=\left.\frac{\partial}{\partial s}\left(s-s_{j}\right)\left(s-s_{j}^{*}\right)\right|_{s=s_{j}}=s_{j}-s_{j}^{*}
$$

and therefore $\gamma_{e j}=1 /\left(s_{j}-s_{j}^{*}\right)$.

Notice that, due to the proportionality of the system, $\boldsymbol{\phi}_{j} \mathbf{D}_{e}(s) \boldsymbol{\phi}_{k}^{T}=0$ when $j \neq k$, for $1 \leq j, k \leq N$. Therefore, pre- and post-multiplying by $\boldsymbol{\Phi}$, the characteristic equation leads to

$$
\operatorname{det}\left[\boldsymbol{\Phi}^{T} \mathbf{D}_{e}(s) \boldsymbol{\Phi}\right]=\prod_{j=1}^{N}\left(s-s_{j}\right)\left(s-s_{j}^{*}\right)=0
$$

which shows that effectively $\left\{s_{j}, s_{j}^{*}\right\}_{j=1}^{N}$ are the complex eigenvalues of the EVM.

The key issue of the following subsections is to show that the TF of the VEM, introduced in Eq. (6.25), can be expressed as the sum of the TF of the EVM, plus a residual term, say $\delta \mathbf{H}(s)$. Thus, mathematically

$$
\mathbf{H}(s)=\mathbf{H}_{e}(s)+\delta \mathbf{H}(s)
$$

Furthermore, the residual term can be bounded in terms of the level of damping and of the proportionality of the viscoelastic matrix. In order to demonstrate this affirmation, the complex eigenvectors and the residues of the VEM will be expanded around their homonymous in the EVM.

\subsubsection{Expansion of the complex eigenvectors}

Each complex eigenvector, can be expressed as a lineal combination of the the modal base $\left\{\phi_{j}\right\}_{j=1}^{N}$ formed by the undamped eigenvectors. Thus, naming $\alpha_{j k}$ the $k$ th coordinate of $\mathbf{u}_{j}$ in that base,

$$
\mathbf{u}_{j}=\sum_{k=1}^{N} \alpha_{j k} \phi_{k}, \quad 1 \leq j \leq N
$$


Without loss of generality, it can be assumed that $\alpha_{j j}=1$ so that

$$
\mathbf{u}_{j}=\phi_{j}+\sum_{\substack{k=1 \\ k \neq j}}^{N} \alpha_{j k} \phi_{k} \equiv \phi_{j}+\delta \mathbf{u}_{j}, \quad 1 \leq j \leq N
$$

Adhikari [28] proposed an efficient procedure for the calculation of $\alpha_{j k}$ for $j \neq k$ using the Neumann expansion method. For the present work purposes, $\alpha_{j k}$ is proportional to the off-diagonal elements of the matrix $\mathbf{G}^{\prime}(s)=\boldsymbol{\Phi}^{T} \mathbf{G}(s) \boldsymbol{\Phi}$. For example, the expression of $\alpha_{j k}$ up to the second order is

$$
\alpha_{j k}=-s_{j} \frac{G_{k j}^{\prime}\left(s_{j}\right)}{\mathcal{D}_{k}\left(s_{j}\right)}+\sum_{\substack{l=1 \\ l \neq j \neq k}}^{N} s_{j}^{2} \frac{G_{k l}^{\prime}\left(s_{j}\right)}{\mathcal{D}_{k}\left(s_{j}\right)} \frac{G_{l j}^{\prime}\left(s_{j}\right)}{\mathcal{D}_{l}\left(s_{j}\right)}
$$

for $1 \leq j \leq N$ with $j \neq k$. A higher order approximation requires adding new terms involving higher order products of the elements $G_{k j}^{\prime}\left(s_{j}\right)$. In the previous expression, the function

$$
\mathcal{D}_{k}(s)=s^{2}+s G_{k k}^{\prime}(s)+\omega_{k}^{2}
$$

is the $k$ th main diagonal element of the dynamic stiffness matrix. As it is known, for proportional systems $G_{j k}^{\prime}(s)=0$ for $j \neq k$. From Eq. (6.37), it is clear that proportionality implies $\alpha_{j k}=0$, for all $1 \leq j, k \leq N$. In general, the reciprocal affirmation is not true, that is, $\alpha_{j k}=0$ does not imply proportionality at least from a theoretical point of view. However, although $\mathbf{G}^{\prime}(s)$ is not diagonal $\forall s$, actually the set of matrices $\left\{\mathbf{G}^{\prime}\left(s_{j}\right)\right\}_{j=1}^{N}$ are diagonal. For more details, see the algorithm for the computation of the values $\alpha_{j k}$ in the work of Adhikari [28]. This reasoning leads in the present paper to the following hypothesis: if $\alpha_{j k}=0,1 \leq j, k \leq N$, $j \neq k$, then $\mathbf{G}^{\prime}(s)$ is diagonal $\forall s \in \mathbb{C}$. Based on this affirmation, an index for measuring the system non-proportionality will later be defined.

\subsubsection{Expansion of the Residues}

This subsection is focused on obtaining a decomposition for the residue of the TF associated to the complex eigenvalue $s_{j}, 1 \leq j \leq N$, residue already presented in Eq. (6.21). The aim is to split $\mathbf{R}_{j}$ into the form

$$
\mathbf{R}_{j}=\mathbf{R}_{e j}+\delta \mathbf{R}_{j}, \quad 1 \leq j \leq N
$$

where $\mathbf{R}_{e j}=\gamma_{e j} \phi_{j} \phi_{j}^{T}$ is the residue in the pole $s_{j}$ of the TF corresponding to the $\mathrm{EVM}$ and $\delta \mathbf{R}_{j}$ is certain residual matrix.

Previously to the calculation of the residue $\mathbf{R}_{j}$, a more detailed expression for $\gamma_{j}$ is developed. For that aim, from Eq. (6.24) we redefine $\gamma_{j}=1 / \rho_{j}$, in which

$$
\rho_{j}=\mathbf{u}_{j}^{T} \frac{\partial \mathbf{D}\left(s_{j}\right)}{\partial s} \mathbf{u}_{j}=\left[\boldsymbol{\phi}_{j}^{T}+\delta \mathbf{u}_{j}^{T}\right]\left[2 s_{j} \mathbf{M}+\overline{\mathbf{G}}\left(s_{j}\right)\right]\left[\boldsymbol{\phi}_{j}+\delta \mathbf{u}_{j}\right]
$$


Eq. (6.36) has been used for the complex eigenvectors and the derivative of the dynamic stiffness matrix $\mathbf{D}(s)$ in $s=s_{j}$ has been evaluated, introducing the nomenclature

$$
\overline{\mathbf{G}}(s)=\mathbf{G}(s)+s \frac{\partial \mathbf{G}}{\partial s}
$$

Thus, expanding the product of the three terms in Eq. (6.40)

$$
\rho_{j}=2 s_{j}+2 s_{j} \sum_{\substack{k=1 \\ k \neq j}}^{N} \alpha_{j k}^{2}\left(\phi_{j}^{T}+2 \delta \mathbf{u}_{j}^{T}\right) \overline{\mathbf{G}}\left(s_{j}\right) \boldsymbol{\phi}_{j}+\delta \mathbf{u}_{j}^{T} \overline{\mathbf{G}}\left(s_{j}\right) \delta \mathbf{u}_{j}
$$

where, for $1 \leq j \leq N$, the following equalities have been used

$$
\begin{aligned}
\phi_{j}^{T} \mathbf{M} \phi_{j} & =1 \\
\phi_{j}^{T} \mathbf{M} \delta \mathbf{u}_{j} & =\sum_{\substack{k=1 \\
k \neq j}}^{N} \alpha_{j k} \phi_{j}^{T} \mathbf{M} \phi_{k}=0 \\
\delta \mathbf{u}_{j}^{T} \mathbf{M} \delta \mathbf{u}_{j} & =\sum_{\substack{k=1 \\
k \neq j}}^{N} \sum_{\substack{l=1 \\
l \neq j}}^{N} \alpha_{j k} \alpha_{j l} \phi_{k}^{T} \mathbf{M} \phi_{l}=\sum_{\substack{k=1 \\
k \neq j}}^{N} \alpha_{j k}^{2}
\end{aligned}
$$

Note that $2 s_{j}$ is equivalent to

$$
2 s_{j}=s_{j}-s_{j}^{*}+s_{j}+s_{j}^{*}=\rho_{e j}+s_{j}+s_{j}^{*}
$$

where the term $\rho_{e j}$ has already been defined in Eq. (6.32). From that, Eq. (6.42) results now in

$$
\rho_{j}=\rho_{e j}+\delta \rho_{j}
$$

being

$$
\delta \rho_{j}=s_{j}+s_{j}^{*}+2 s_{j} \sum_{\substack{k=1 \\ k \neq j}}^{N} \alpha_{j k}^{2}+\left(\phi_{j}^{T}+2 \delta \mathbf{u}_{j}^{T}\right) \overline{\mathbf{G}}\left(s_{j}\right) \phi_{j}+\delta \mathbf{u}_{j}^{T} \overline{\mathbf{G}}\left(s_{j}\right) \delta \mathbf{u}_{j}
$$

We will turn to this expanded version of $\delta \rho_{j}$ in the next section. To calculate the residue, only Eq. (6.45) is relevant, thus, $\gamma_{j}$ may be expressed as function of the above results

$$
\gamma_{j}=\frac{1}{\rho_{j}}=\frac{1}{\rho_{e j}+\delta \rho_{j}}=\gamma_{e j}\left(1-\frac{\epsilon_{j}}{1+\epsilon_{j}}\right)
$$

where $\gamma_{e j}=1 / \rho_{e j}$ and $\epsilon_{j}=\delta \rho_{j} / \rho_{e j}$ 
The conditions for expanding $\mathbf{R}_{j}$ are set since the expressions for $\gamma_{j}$ and for $\mathbf{u}_{j}$ are available in Eqs. (6.47) and (6.36), respectively. Thus, entering in the definition of $\mathbf{R}_{j}$

$$
\begin{aligned}
\mathbf{R}_{j} & =\gamma_{j} \mathbf{u}_{j} \mathbf{u}_{j}^{T} \\
& =\gamma_{e j}\left(1-\frac{\epsilon_{j}}{1+\epsilon_{j}}\right)\left(\phi_{j}+\delta \mathbf{u}_{j}\right)\left(\phi_{j}^{T}+\delta \mathbf{u}_{j}^{T}\right) \\
& =\gamma_{e j} \phi_{j} \phi_{j}^{T}+\delta \mathbf{R}_{j}
\end{aligned}
$$

where the residual matrix defined in Eq. (6.39) adopts the form

$$
\delta \mathbf{R}_{j}=\frac{\gamma_{e j}}{1+\epsilon_{j}}\left(\delta \mathbf{u}_{j} \phi_{j}^{T}+\phi_{j} \delta \mathbf{u}_{j}^{T}+\delta \mathbf{u}_{j} \delta \mathbf{u}_{j}^{T}-\epsilon_{j} \phi_{j} \phi_{j}^{T}\right)
$$

Note that the previous expansion has been developed for the complex modes, i.e. $1 \leq j \leq N$. As it will be later shown, the residue associated with the nonviscous modes, $2 N+1 \leq j \leq m$ will not be expanded, since this residue can be considered itself residual. This is so because, in general, non-viscous modes in the damped response are not relevant with respect to the exact solution, [28, 30]. In the next subsection, the relationship between the TF of the VEM and the $\mathrm{TF}$ of the EVM will be presented. Also it will be demonstrated that the former relationship can be expressed as the sum of the latter plus a residual term.

\subsubsection{Expansion of the TF}

Rewriting the TF of the VEM given in Eq. (6.25) and introducing the Eq. (6.48), results

$\mathbf{H}(s)=\sum_{j=1}^{N}\left[\frac{\gamma_{e j} \boldsymbol{\phi}_{j} \boldsymbol{\phi}_{j}^{T}+\delta \mathbf{R}_{j}}{s-s_{j}}+\frac{\gamma_{e j}^{*} \boldsymbol{\phi}_{j} \boldsymbol{\phi}_{j}^{T}+\delta \mathbf{R}_{j}^{*}}{s-s_{j}^{*}}\right]+\sum_{j=2 N+1}^{m} \frac{\mathbf{R}_{j}}{s-s_{j}} \equiv \mathbf{H}_{e}(s)+\delta \mathbf{H}(s)$

$\mathbf{H}_{e}(s)$ being the TF of the EVM, defined in Eq. (6.26), and

$$
\begin{aligned}
\delta \mathbf{H}(s) & =\sum_{j=1}^{N}\left[\frac{\delta \mathbf{R}_{j}}{s-s_{j}}+\frac{\delta \mathbf{R}_{j}^{*}}{s-s_{j}^{*}}\right]+\sum_{j=2 N+1}^{m} \frac{\mathbf{R}_{j}}{s-s_{j}} \\
& \equiv \delta \mathbf{H}_{c}(s)+\delta \mathbf{H}_{r}(s)
\end{aligned}
$$

a residual matrix which will be named Residual Transfer Function (RTF), whose expression is separated in two terms. The first, $\delta \mathbf{H}_{c}(s)$, includes the part corresponding to the complex modes. From Eq. (6.49), the matrices $\delta \mathbf{R}_{j}$ are proportional to $\delta \mathbf{u}_{j}$ and $\delta \rho_{j}$. If $\delta \mathbf{u}_{j}$ with respect to $\phi_{j}$ and $\delta \rho_{j}$ with respect to $\rho_{e j}$ may be considered negligible, then it will be expected that also $\delta \mathbf{H}_{c}(s)$ will have a small relevance compared with $\mathbf{H}_{e}(s)$. The second term, $\delta \mathbf{H}_{r}(s)$, corresponds to the 
nonviscous modes. Note that $\delta \mathbf{H}_{r}(s)$ coincides completely with the non-complex part in the TF.

As mentioned before, in the majority of the physical systems, the effect of nonviscous modes in the response can be neglected with respect to that of the complex modes. Therefore, it makes sense to define $\delta \mathbf{H}_{c}(s)$ as a residual term. More details on the role played by the nonviscous modes can be found in the works of Woodhouse [30] and Adhikari [28, 32].

Eq. (6.50) shows clearly that the TF of the viscoelastic system can be approximated by the TF of the EVM provided that the RTF, $\delta \mathbf{H}(s)$, does not become too important. The next section is focused in giving an upper bound of the RTF showing that this bound will depend, on one hand, on the proportionality of the system, and on the other, on its level of damping.

\subsection{Bound of the Residual Transfer Function}

\subsubsection{Indexes of non-proportionality and damping}

In this section two indexes to: (i) measure the proportionality of the system and (ii) the damping induced by the matrix $\mathbf{G}(s)$, are defined. In the bibliography, different non-proportionality indexes can be found, for instance, Adhikari [105] made a survey of the most important works, proposing in turn a new index based on the normalized distance between the optimal complex modes and the undamped ones. Using the same principle, another index is developed in the present paper that will be used to express the bound of the RTF in terms of the non-proportionality of the system. As it has been shown in subsection 6.3.2, there exists a direct relationship between the coefficients $\alpha_{j k}$ and the non-proportionality of the system. This motivates the introduction of the following non-dimensional index

$$
\alpha=\|\mathbf{A}\|=\sum_{j=1}^{N} \sum_{k=1}^{N}\left|A_{j k}\right|
$$

where the elements of the matrix $\mathbf{A}$ are

$$
A_{j k}= \begin{cases}\alpha_{j k} & j \neq k \\ 0 & j=k\end{cases}
$$

According with the definition of $\alpha$, proportional systems are characterized by having $\alpha=0$. 
For the purposes of this research, it is important to evaluate whether the viscoelastic matrix $\mathbf{G}(s)$ induces a strongly or a lightly damped response. For that purpose, a new damping index is introduced by

$$
\beta=\frac{1}{\Omega\|\mathbf{M}\|} \sum_{j=1}^{N}\left(\left\|\mathbf{G}\left(s_{j}\right)\right\|+\left|s_{j}\right|\left\|\frac{\partial \mathbf{G}\left(s_{j}\right)}{\partial s}\right\|\right)
$$

that is non-dimensional and in which $\Omega=\sum_{j=1}^{N}\left|s_{j}\right|$.

It is important to remark two aspects with respect to this new index $\beta$. First, although in an undamped system obviously $\beta=0$, damping functions $\mathbf{G}(s)$ that are not identically null and vanish in the set $\left\{s_{j}\right\}_{j=1}^{N}$ may exist. It is clear that such functions are not of interest for real physical systems since they would present strong discontinuities close to the eigenvalues. Therefore, the following hypothesis will be assumed: if $\beta=0$, then $\mathbf{G}(s) \equiv \mathbf{0}$. Second, undamped systems are proportional, i.e., $\beta=0$ implies $\alpha=0$. But the opposite is in general not true, fact that constitutes the main reason to use the two different indexes. This duality allows to express the RTF as a function of each index separately.

\subsubsection{Upper Bound Computation}

In the previous section, it has been demonstrated that the TF of the viscoelastic system can be expressed as

$$
\mathbf{H}(s)=\mathbf{H}_{e}(s)+\delta \mathbf{H}(s)=\mathbf{H}_{e}(s)+\delta \mathbf{H}_{c}(s)+\delta \mathbf{H}_{r}(s)
$$

Reordering the TF's in the left part of the equation and taking norms

$$
\left\|\mathbf{H}(s)-\mathbf{H}_{e}(s)\right\|=\|\delta \mathbf{H}(s)\| \leq\left\|\delta \mathbf{H}_{c}(s)\right\|+\left\|\delta \mathbf{H}_{r}(s)\right\|
$$

The objective of this subsection is to find an upper bound of the $\operatorname{RTF}\|\delta \mathbf{H}(s)\|$, as a function of the indexes defined in the previous subsection. Previously, it is necessary to prove the following four lemmas.

Lemma 1. Let $\delta \mathbf{u}_{j}$ with $1 \leq j \leq N$ be, the residual eigenvector defined in the expression (6.36). Then,

$$
\sum_{j=1}^{N}\left\|\delta \mathbf{u}_{j}\right\| \leq \alpha\|\boldsymbol{\Phi}\|
$$

where $\boldsymbol{\Phi}$ is the matrix that contains the undamped eigenvectors in columns and $\alpha$ the non-proportionality index.

Lemma 2. Let $\left\{s_{j}, s_{j}^{*}\right\}_{j=1}^{N}$ be the set of complex eigenvalues, then

$$
\sum_{j=1}^{N}\left|s_{j}+s_{j}^{*}\right| \leq 2 \gamma \Omega^{2}\|\mathbf{\Phi}\|^{2}\|\mathbf{M}\|(1+\alpha) \beta
$$


where $\Omega$ was defined before and

$$
\gamma=\sum_{j=1}^{N}\left|\gamma_{e j}\right|=\sum_{j=1}^{N} \frac{1}{\left|s_{j}-s_{j}^{*}\right|}
$$

Lemma 3. Let $\delta \rho_{j}$ and $\epsilon_{j}$ be the magnitudes defined in Eq. (6.46) and (6.47) and $\rho_{e j}=1 / \gamma_{e j}=s_{j}-s_{j}^{*}$. Then

$$
\sum_{j=1}^{N}\left|\epsilon_{j}\right| \leq\|\mathbf{\Phi}\|^{2}\|\mathbf{M}\| \gamma \Omega\left[\frac{2 \alpha^{2}}{N}+\beta(1+\alpha)(1+\alpha+2 \gamma \Omega)\right]
$$

Lemma 4. Let $s_{j}(\beta) \in \mathbb{R}$ be the $j$ th nonviscous root, with $2 N+1 \leq j \leq m$ for a fixed value of the damping index $\beta$. Let $\mathcal{P}=\left\{p_{1}, \cdots, p_{h}\right\}$ the set of real poles of the function $\mathbf{G}(s)$, i.e., $p_{l} \in \mathbb{R}$ for $1 \leq l \leq h$. Then

$$
\lim _{\beta \rightarrow 0} s_{j} \in \mathcal{P}
$$

The proofs of these Lemmas can be found in the Appendices 6.A to 6.D. Now, the following Theorem concerning the bounding of the distance between the Transfer Functions of VEM and EVM can be enunciated.

Theorem 1. Let $\mathbf{H}(s)$ and $\mathbf{H}_{e}(s)$ be the TF's of the VEM and of the EVM, defined in Eqs. (6.25) and (6.26), respectively. Then, the error between the TF of the VEM and that of the EVM is bounded by

$$
\left\|\mathbf{H}(s)-\mathbf{H}_{e}(s)\right\| \leq \frac{1}{N}\|\mathbf{\Phi}\|^{2}\|\mathbf{M}\| \mathcal{L}(\alpha, \beta) \mathcal{H}(s)
$$

where

$$
\mathcal{H}(s)=\sum_{j=1}^{m} \frac{\gamma\|\boldsymbol{\Phi}\|^{2}}{\left|s-s_{j}\right|}
$$

and $\mathcal{L}(\alpha, \beta)$ is certain function of non-proportionality and damping indexes, with the following properties for $\alpha>0, \beta>0$

i) $\mathcal{L}(\alpha, \beta)$ is a positive and increasing function

ii) $\lim _{(\alpha, \beta) \rightarrow(0,0)} \mathcal{L}(\alpha, \beta)=0$

iii) $\mathcal{L}(0, \beta)<\mathcal{L}(\alpha, \beta)$ for $\alpha>0, \beta>0$

Proof. Rewriting the RTF expression

$$
\left\|\mathbf{H}(s)-\mathbf{H}_{e}(s)\right\| \leq\left\|\delta \mathbf{H}_{c}(s)\right\|+\left\|\delta \mathbf{H}_{r}(s)\right\|
$$


the computation of the requested upper bound requires in turn bounding the norm of the matrices $\delta \mathbf{H}_{c}(s)$ and $\delta \mathbf{H}_{r}(s)$ as defined in Eq. (6.51).

In the first place, the norm of $\delta \mathbf{H}_{c}(s)$ will be evaluated

$$
\begin{aligned}
\left\|\delta \mathbf{H}_{c}(s)\right\| & =\left\|\sum_{j=1}^{N}\left(\frac{\delta \mathbf{R}_{j}}{s-s_{j}}+\frac{\delta \mathbf{R}_{j}^{*}}{s-s_{j}^{*}}\right)\right\| \leq \sum_{j=1}^{N}\left\|\frac{\delta \mathbf{R}_{j}}{s-s_{j}}\right\|+\sum_{j=1}^{N}\left\|\frac{\delta \mathbf{R}_{j}^{*}}{s-s_{j}^{*}}\right\| \\
& \leq\left(\sum_{j=1}^{N} \frac{1}{\left|s-s_{j}\right|}+\frac{1}{\left|s-s_{j}^{*}\right|}\right) \sum_{j=1}^{N}\left\|\delta \mathbf{R}_{j}\right\|
\end{aligned}
$$

Taking norms of $\delta \mathbf{R}_{j}$ from Eq. (6.49), using the results of Lemmas 1 and 3 , the following inequality can be obtained

$$
\begin{aligned}
\sum_{j=1}^{N}\left\|\delta \mathbf{R}_{j}\right\| & =\sum_{j=1}^{N}\left|\frac{\gamma_{e j}}{1+\epsilon_{j}}\right|\left\|\delta \mathbf{u}_{j} \phi_{j}^{T}+\phi_{j} \delta \mathbf{u}_{j}^{T}+\delta \mathbf{u}_{j} \delta \mathbf{u}_{j}^{T}-\epsilon_{j} \phi_{j} \boldsymbol{\phi}_{j}^{T}\right\| \\
& \leq \gamma \mu\|\boldsymbol{\Phi}\|^{2}\left(\alpha+\alpha+\alpha^{2}+\sum_{j=1}^{N}\left|\epsilon_{j}\right|\right) \\
& \leq \gamma \mu\|\boldsymbol{\Phi}\|^{4}\|\mathbf{M}\|\left[\frac{\alpha(2+\alpha)}{N}+\gamma \Omega \frac{2 \alpha^{2}}{N}+\beta \gamma \Omega(1+\alpha)(1+\alpha+2 \gamma \Omega)\right]
\end{aligned}
$$

where the following coefficient has been introduced

$$
\mu=\sum_{j=1}^{N} \frac{1}{\left|1+\epsilon_{j}\right|}
$$

Finally, entering in the expression (6.65) the RTF bound corresponding to the complex modes can be written in the form

$$
\left\|\delta \mathbf{H}_{c}(s)\right\| \leq \frac{1}{N}\|\mathbf{\Phi}\|^{2}\|\mathbf{M}\| \mathcal{L}_{c}(\alpha, \beta) \mathcal{H}_{c}(s)
$$

where

$$
\mathcal{L}_{c}(\alpha, \beta)=\alpha(2+\alpha+2 \alpha \gamma \Omega) \mu+\beta(1+\alpha)(1+\alpha+2 \gamma \Omega) \gamma \Omega N \mu
$$

and

$$
\mathcal{H}_{c}(s)=\gamma\|\boldsymbol{\Phi}\|^{2} \sum_{j=1}^{N}\left(\frac{1}{\left|s-s_{j}\right|}+\frac{1}{\left|s-s_{j}^{*}\right|}\right)
$$


Secondly, to bound the RTF related to the nonviscous modes, $\left\|\delta \mathbf{H}_{r}(s)\right\|$, it is necessary to evaluate the $j$ th residue norm, $\left\|\mathbf{R}_{j}\right\|$, for $2 N+1 \leq j \leq m$. Indirectly, the $j$ th nonviscous eigenvalue depends on the damping level through the index $\beta$. As a consequence, both the associated eigenvector $\mathbf{u}_{j}(\beta)$ and the variable $\gamma_{j}(\beta)$ are functions of $\beta$. The latter variable can be expanded by Taylor series around $\beta=0$

$$
\gamma_{j}(\beta)=\gamma_{j}(0)+\frac{\partial \gamma_{j}(0)}{\partial \beta} \beta+\frac{1}{2} \frac{\partial^{2} \gamma_{j}(0)}{\partial \beta^{2}} \beta^{2}+\cdots, \quad \beta>0
$$

where the values $\gamma_{j}(0), \partial \gamma_{j}(0) / \partial \beta, \ldots$ must be considered limits when $\beta \rightarrow 0$. In particular, we are going to prove that $\gamma_{j}(0)=0$ : from Eq. (6.24) the following limit can be set

$$
\lim _{\beta \rightarrow 0} \frac{1}{\left|\gamma_{j}(\beta)\right|}=\lim _{\beta \rightarrow 0}\left|\mathbf{u}_{j} \frac{\partial \mathbf{D}\left(s_{j}\right)}{\partial s} \mathbf{u}_{j}^{T}\right|
$$

Using Lemma $4, \lim _{\beta \rightarrow 0} s_{j}=\sigma_{j}$ is a pole of $\mathbf{G}(s)$ and, as a consequence, also of $\partial \mathbf{G}(s) / \partial s$. Therefore

$$
\lim _{\beta \rightarrow 0}\left|\mathbf{u}_{j} \frac{\partial \mathbf{D}\left(s_{j}\right)}{\partial s} \mathbf{u}_{j}^{T}\right|=\left|\mathbf{u}_{j}\left(2 \sigma_{j}+\mathbf{G}\left(\sigma_{j}\right)+\sigma_{j} \frac{\partial \mathbf{G}\left(\sigma_{j}\right)}{\partial s}\right) \mathbf{u}_{j}^{T}\right|=+\infty
$$

From here, $\lim _{\beta \rightarrow 0}\left|\gamma_{j}(\beta)\right|=0$, that leads to $\gamma_{j}(0)=0$. This conclusion means that the coefficient $\left|\gamma_{j}\right|$ can be simplified to the product of $\beta$ and another term, that will be normalized with the variable $\gamma$ introduced in Eq. (6.59)

$$
\left|\gamma_{j}\right|=\beta \psi_{j}(\beta) \gamma, \quad 2 N+1 \leq j \leq m
$$

$\psi_{j}(\beta)$ being a certain non-dimensional positive function of $\beta$. Under these considerations, the problem of evaluating the sum can be solved with

$$
\begin{aligned}
\sum_{j=2 N+1}^{m}\left\|\mathbf{R}_{j}\right\| & =\sum_{j=2 N+1}^{m}\left|\gamma_{j}\right|\left\|\mathbf{u}_{j} \mathbf{u}_{j}^{T}\right\|=\sum_{j=2 N+1}^{m} \beta \psi_{j}(\beta) \gamma\left\|\mathbf{u}_{j} \mathbf{u}_{j}^{T}\right\| \\
& =\frac{1}{N}\|\mathbf{\Phi}\|^{4}\|\mathbf{M}\| \beta \psi(\beta) \gamma
\end{aligned}
$$

where the new function is

$$
\psi(\beta)=\frac{N}{\|\boldsymbol{\Phi}\|^{4}\|\mathbf{M}\|} \sum_{j=2 N+1}^{m} \psi_{j}(\beta)\left\|\mathbf{u}_{j} \mathbf{u}_{j}^{T}\right\|
$$

that has been introduced with the objective to find an analog expression for $\left\|\delta \mathbf{H}_{r}(s)\right\|$ to that given in Eq (6.68). The RTF bound corresponding to the non- 
viscous modes can be calculated as

$$
\begin{aligned}
\left\|\delta \mathbf{H}_{r}(s)\right\| & =\left\|\sum_{j=2 N+1}^{m} \frac{\mathbf{R}_{j}}{s-s_{j}}\right\| \leq \sum_{j=2 N+1}^{m}\left\|\frac{\mathbf{R}_{j}}{s-s_{j}}\right\| \\
& \leq\left(\sum_{j=2 N+1}^{m} \frac{1}{\left|s-s_{j}\right|}\right) \sum_{j=2 N+1}^{m}\left\|\mathbf{R}_{j}\right\| \\
& =\frac{1}{N}\|\mathbf{\Phi}\|^{2}\|\mathbf{M}\| \beta \psi(\beta) \sum_{j=2 N+1}^{m} \frac{\gamma\|\mathbf{\Phi}\|^{2}}{\left|s-s_{j}\right|} \\
& \equiv \frac{1}{N}\|\mathbf{\Phi}\|^{2}\|\mathbf{M}\| \mathcal{L}_{r}(\beta) \mathcal{H}_{r}(s)
\end{aligned}
$$

where

$$
\mathcal{L}_{r}(\beta)=\beta \psi(\beta)
$$

and

$$
\mathcal{H}_{r}(s)=\sum_{j=2 N+1}^{m} \frac{\gamma\|\boldsymbol{\Phi}\|^{2}}{\left|s-s_{j}\right|}
$$

Finally, assembling the results from Eqs. (6.68) and (6.77), the distance between both TF's can be bounded by

$$
\left\|\mathbf{H}(s)-\mathbf{H}_{e}(s)\right\| \leq \frac{1}{N}\|\mathbf{\Phi}\|^{2}\|\mathbf{M}\| \mathcal{L}(\alpha, \beta) \mathcal{H}(s)
$$

with

$$
\begin{aligned}
\mathcal{L}(\alpha, \beta) & =\mathcal{L}_{c}(\alpha, \beta)+\mathcal{L}_{r}(\beta) \\
& =\mu[\alpha(2+\alpha+2 \alpha \gamma \Omega)+\beta(1+\alpha)(1+\alpha+2 \gamma \Omega) \gamma \Omega N]+\beta \psi(\beta)
\end{aligned}
$$

and

$$
\mathcal{H}(s)=\mathcal{H}_{c}(s)+\mathcal{H}_{r}(s)=\sum_{j=1}^{m} \frac{\gamma\|\boldsymbol{\Phi}\|^{2}}{\left|s-s_{j}\right|}
$$

Observing the function $\mathcal{L}(\alpha, \beta)$, property $i$ ) holds immediately, since all its terms involved are positive. The second item, can also be proved directly, simply evaluating $\mathcal{L}(0,0)=0$. To prove the third, $\mathcal{L}(\alpha, \beta)$ can be reordered as

$$
\mathcal{L}(\alpha, \beta)=\mathcal{L}(0, \beta)+\alpha \mathcal{M}(\alpha, \beta)
$$

the function being

$$
\mathcal{M}(\alpha, \beta)=\mu[2+\alpha+\alpha(2+N \beta) \gamma \Omega+2 N \beta \gamma \Omega(1+\gamma \Omega)]
$$


strictly positive $\forall \alpha>0, \beta>0$. Hence, from Eq. (6.83), it follows directly that $\mathcal{L}(\alpha, \beta)>\mathcal{L}(0, \beta)$.

We believe that this Theorem is the main contribution of this paper. Not only an equivalent model of pure viscous nature is proposed to study symmetric viscoelastic models, but also an error bound in the evaluation of the transfer functions is calculated. This bound gives important qualitative information: first, the study of systems lightly damped, will produce lower error than that of strongly damped. Second, the lower the non-proportionality of the system, the better the approximation made by the EVM. In particular, through observation of the bound expression Eq (6.80), it is expected that the EVM will be a close approximation for proportional systems, for which $\alpha=0$. Numerical examples presented in the next section will allow us to validate the proposed method, evaluating the influence of the indexes $\alpha, \beta$.

\subsection{Numerical Examples}

\subsubsection{Description}

To illustrate the suitability of the proposed EVM, three numerical examples are analyzed. In them, the non-proportionality of the system as well as the level of damping vary. The three models consist on a six-dof lumped-mass system with three different dampers distributions, as shown in Figure 6.1. Masses $m$ are linked through linear and elastic springs with rigidities $k$. Thus, the mass matrix is $\mathbf{M}=m \mathbf{I}_{6}$ and the stiffness

$$
\mathbf{K}=k\left[\begin{array}{cccccc}
2 & -1 & 0 & 0 & 0 & 0 \\
-1 & 2 & -1 & 0 & 0 & 0 \\
0 & -1 & 2 & -1 & 0 & 0 \\
0 & 0 & -1 & 2 & -1 & 0 \\
0 & 0 & 0 & -1 & 2 & -1 \\
0 & 0 & 0 & 0 & -1 & 2
\end{array}\right]
$$

The damping is introduced through viscoelastic dampers connecting consecutive masses. For each damper, the same viscoelastic model based on a double exponential kernel function is used with the form

$$
\mathcal{G}(t)=c_{v} \frac{1}{2}\left(\mu_{1} e^{-\mu_{1} t}+\mu_{2} e^{-\mu_{2} t}\right)
$$

is employed, where the parameters $\mu_{1}, \mu_{2}$ are directly related with the nonviscousness of the system. Small values of these parameters induce highly nonviscous behavior, whereas large values correspond to a near-viscously damped system with coefficient $c_{v}$. For this reason, this coefficient may be written in the 
form $c_{v}=2 m \omega_{0} \zeta$, with $\omega_{0}=\sqrt{k / m}$ a reference frequency and $\zeta$ a dimensionless damping ratio whose magnitude measures the level of damping induced. Taking the Laplace Transform, the viscoelastic function in the variable $s$ is

$$
G(s)=m \omega_{0} \zeta\left(\frac{\mu_{1}}{s+\mu_{1}}+\frac{\mu_{2}}{s+\mu_{2}}\right)
$$

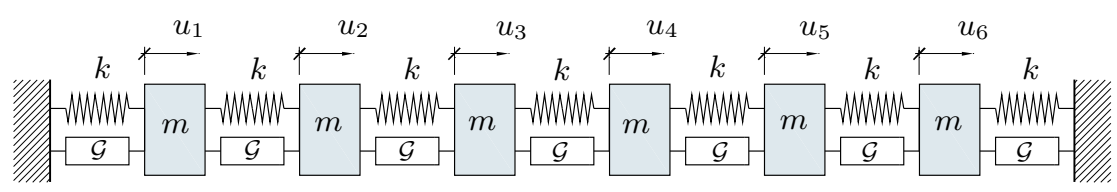

Example 1
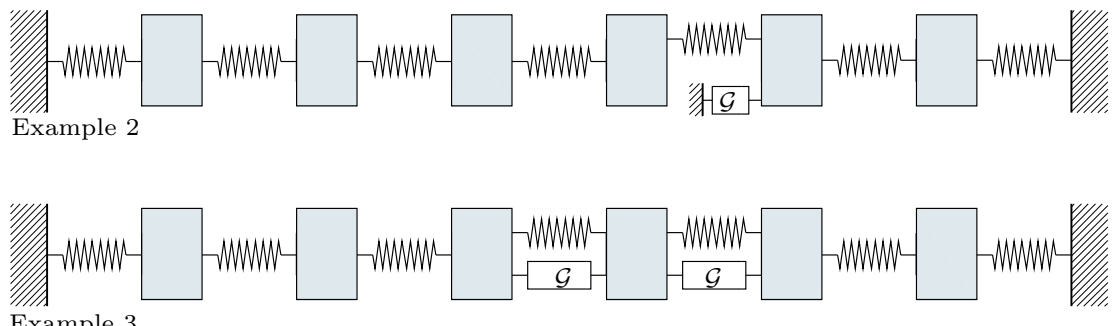

Figure 6.1: Structural configuration of 6-dof examples. $m=10^{3} \mathrm{~kg}, k=10^{5} \mathrm{~N} / \mathrm{m}$

The viscoelastic matrix can be described in the form $\mathbf{G}(s)=G(s) \mathbf{Q}$, where the matrix $\mathbf{Q}$ takes the following values for each example

- Example 1

$$
\mathbf{Q}=\left[\begin{array}{cccccc}
2 & -1 & 0 & 0 & 0 & 0 \\
-1 & 2 & -1 & 0 & 0 & 0 \\
0 & -1 & 2 & -1 & 0 & 0 \\
0 & 0 & -1 & 2 & -1 & 0 \\
0 & 0 & 0 & -1 & 2 & -1 \\
0 & 0 & 0 & 0 & -1 & 2
\end{array}\right]
$$

- Example 2

$$
\mathbf{Q}=\left[\begin{array}{llllll}
0 & 0 & 0 & 0 & 0 & 0 \\
0 & 0 & 0 & 0 & 0 & 0 \\
0 & 0 & 0 & 0 & 0 & 0 \\
0 & 0 & 0 & 0 & 0 & 0 \\
0 & 0 & 0 & 0 & 1 & 0 \\
0 & 0 & 0 & 0 & 0 & 0
\end{array}\right]
$$




\begin{tabular}{ccccc} 
& Case & $\zeta$ & $\alpha$ & $\beta$ \\
\hline Example 1 & $\mathrm{a}$ & 0.01 & 0.000 & 0.102 \\
& $\mathrm{~b}$ & 0.20 & 0.000 & 0.911 \\
\hline Example 2 & $\mathrm{a}$ & 0.01 & 0.097 & 0.005 \\
& $\mathrm{~b}$ & 0.20 & 1.009 & 0.047 \\
\hline Example 3 & $\mathrm{a}$ & 0.01 & 0.214 & 0.037 \\
& $\mathrm{~b}$ & 0.20 & 1.640 & 0.359 \\
\hline
\end{tabular}

Table 6.1: Non-proportionality and damping indexes for the three examples shown in the Fig. 6.1

- Example 3

$$
\mathbf{Q}=\left[\begin{array}{cccccc}
0 & 0 & 0 & 0 & 0 & 0 \\
0 & 0 & 0 & 0 & 0 & 0 \\
0 & 0 & 1 & -1 & 0 & 0 \\
0 & 0 & -1 & 2 & -1 & 0 \\
0 & 0 & 0 & -1 & 1 & 0 \\
0 & 0 & 0 & 0 & 0 & 0
\end{array}\right]
$$

In addition, each example is divided into two cases to study the level of damping influence with the $\zeta$-parameter. Thus, two values are considered, $\zeta=0.01$ to model light damping, and $\zeta=0.2$ for strong damping. The rest of parameters remain invariable taking the values $m=5000 \mathrm{~kg}, k=5 \times 10^{5} \mathrm{~N} / \mathrm{m}, \mu_{1}=5 \mathrm{rad} / \mathrm{s}$ and $\mu_{2}=15 \mathrm{rad} / \mathrm{s}$. Solving the characteristic equation for each example, the set of eigenvalues and eigenvectors can be extracted, the complex and the real ones. Based on the definitions given in Eqs. (6.52) and (6.54), the corresponding $\alpha-$ and $\beta$-indexes can be computed. The results are shown in Table 6.1. Related to the results obtained for these indexes some remarks must be made. First, it can be noted that the $\beta$-index becomes a measure of the system global damping. Therefore, smallest values of $\zeta$ will correspond to smallest values of $\beta$ only if the number of dampers involved is small and vice versa. This explains why, the Example 1b presents the highest value whereas the Example 2a the lowest one.

Second, the structural configuration of Examples 2 and 3 has been chosen so that a fixed value of the parameter $\zeta$, forces that the first is always more proportional, that is, its $\alpha$-index is smaller than that of the second example. This last example can be considered the least proportional among the three.

With the objective of comparing the results of the TF calculated from the EVM and from the VEM, plots of the TF matrix entry $(2,4)$ in absolute value are showed in Figs. 6.2,6.3. The curves are dimensionless, representing the matrix entry $\mathbf{h}(i \omega)=m \omega_{0}^{2} \mathbf{H}(i \omega)$ vs. the normalized frequency $\omega / \omega_{0}$. 

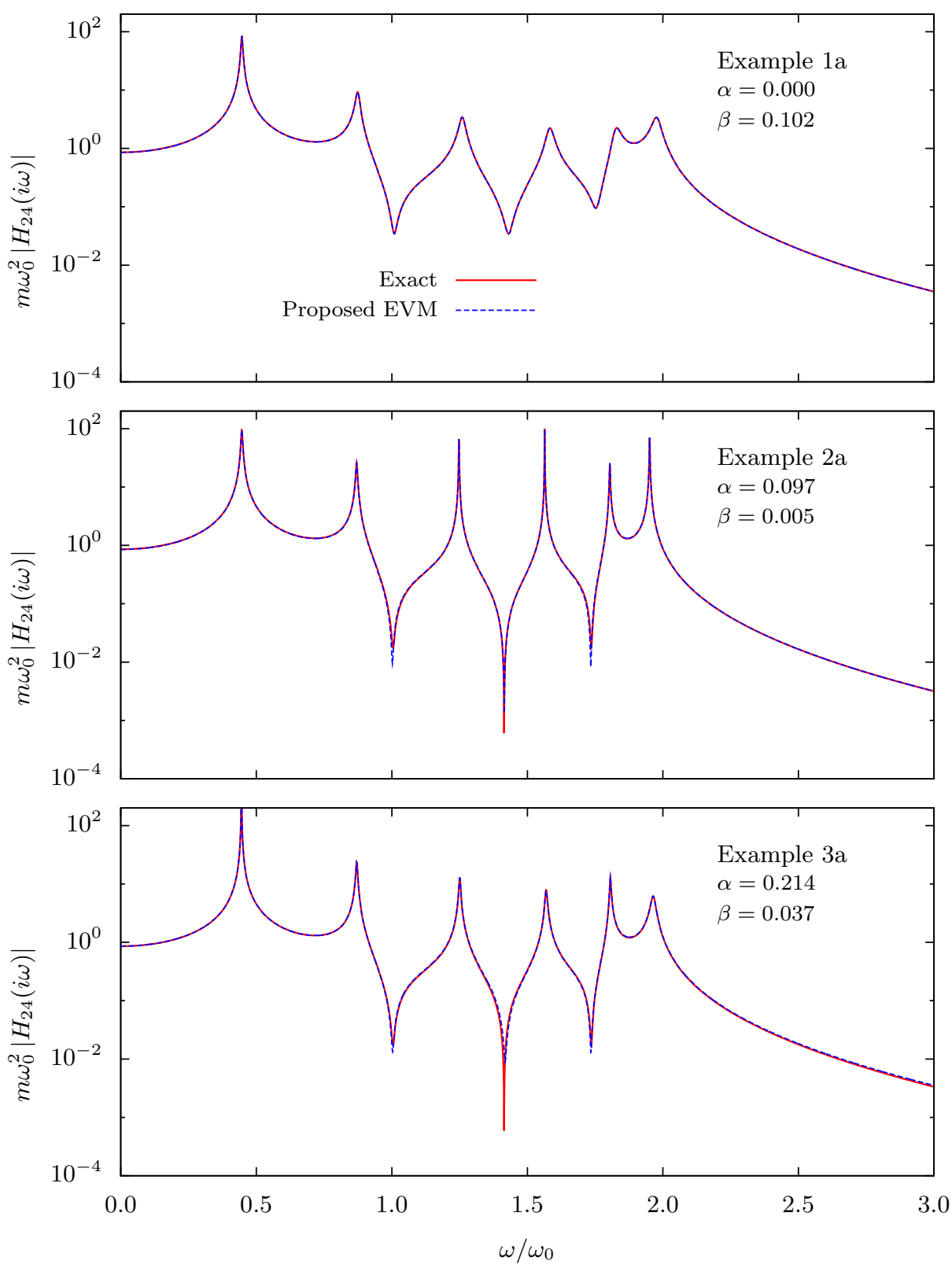

Figure 6.2: Representation of the absolute value of the dimensionless TF's $m \omega_{0}^{2} H_{24}(i \omega)$ and $m \omega_{0}^{2} H_{e, 24}(i \omega)$. (-) Exact. (- - ) Approximation by EVM 

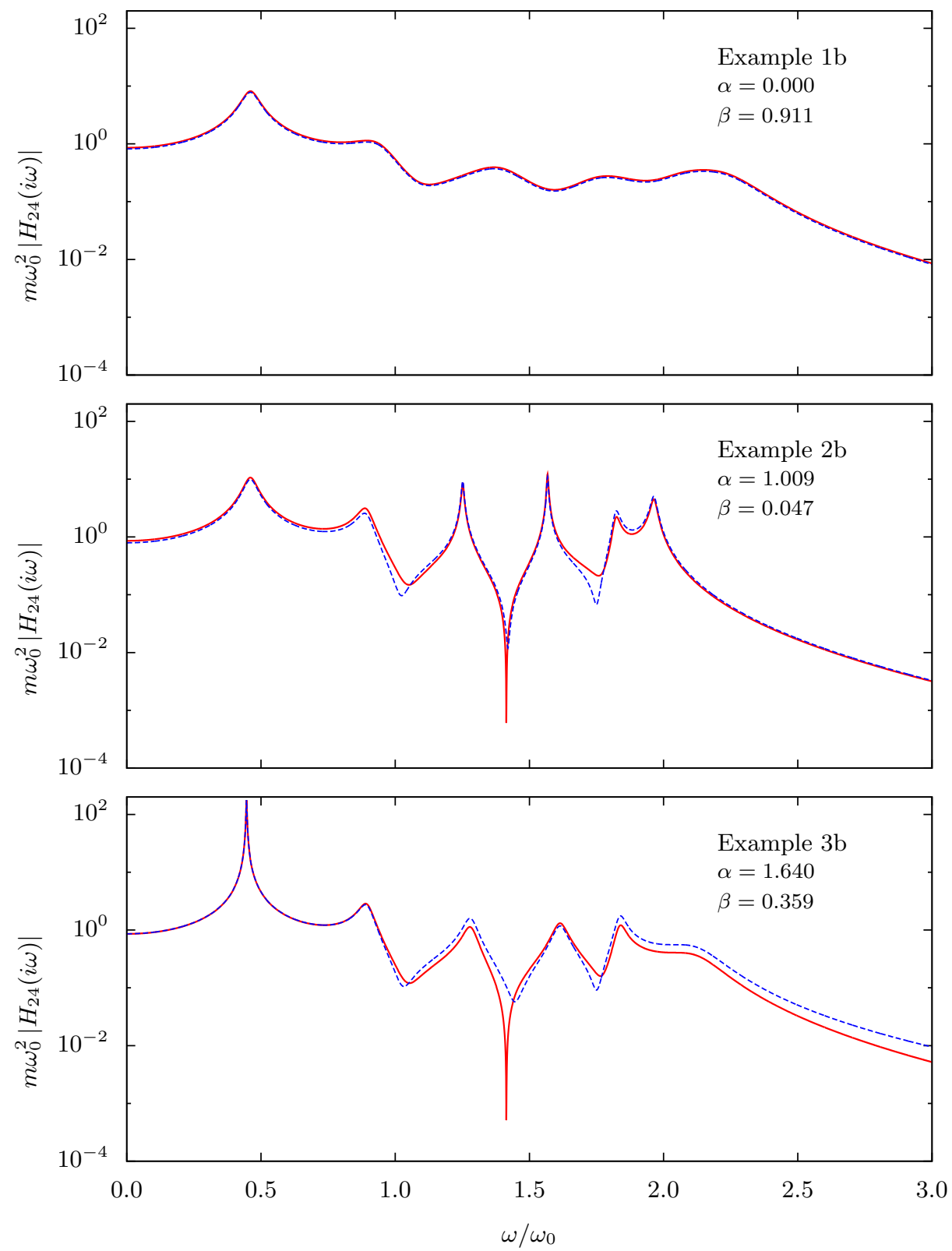

Figure 6.3: Representation of the absolute value of the dimensionless TF's $m \omega_{0}^{2} H_{24}(i \omega)$ and $m \omega_{0}^{2} H_{e, 24}(i \omega)$. (-) Exact. (- - ) Approximation by EVM 
As expected, the approximation from EVM is very close to the exact VEM for both Cases of Example 1. Notice that this example represents a proportional system with $\alpha=0$. The Examples 2 and 3 show that the higher the loss of proportionality, the greater the error in the evaluation of the TF. However, the EVM still produces very good approximations provided that the system is lightly damped as in Cases 2a, 3a. The difference between both results becomes important at frequencies near to the anti-resonances. The reason could be related with the coincidence of the complex eigenvalues for the EVM and the VEM. At the minimums far from these frequencies the accuracy will be lowest. In particular, the highest difference appears at the anti-resonance corresponding to the modes activated by the damper location, $\omega / \omega_{0} \approx 1.4$.

In any case, for all Examples the TF estimated with the EVM presents good agreement with the exact $\mathrm{TF}$ in a wide range of frequencies, even for Example $3 \mathrm{~b}$ whereby high non-proportionality and strong damping are present. Furthermore, the resonance peaks of Examples 2 and 3 also are in agreement. Therefore, it is clear that the response evaluated with the proposed EVM gives a good estimation of the real response.

\subsection{Conclusions}

Linear viscoelastic systems are featured by having dissipative forces that depend on the history of the velocity degrees of freedom, via matrix kernel functions. In this work, an equivalent linear viscously damped system, named Equivalent Viscous Model (EVM), is proposed. The construction of this model requires the set of complex eigenvalues and the complete solution of the undamped eigenproblem. It has been demonstrated that the exact Transfer Function (TF) of the original viscoelastic model (VEM) can be expanded and expressed as the sum of the TF of the EVM plus a residual term.

The accuracy given by the approximated TF of the EVM respect to the exact VEM directly depends on the quantification of the system damping and, in addition, on the proportionality of the viscoelastic matrix. To quantify both properties, two indexes are defined. The first, named non-proportionality index, is related to the complex eigenvector components in the modal base. The second, named damping index, is defined from the viscoelastic matrix function and its derivative, evaluated in the complex eigenvalues. The theoretical results have shown that the residual norm is bounded by an expression that depends on both indexes. This bounding implies that systems lightly damped (with matrix $\mathbf{G}^{\prime}(s)$ diagonally dominant) will present less error in the estimation of the TF through the EVM. 
Finally, the theoretical results are validated by numerical examples, consisting on three discrete systems with six degrees of freedom each. Different configurations of the viscoelastic constraint allows to analyze the results as function of the defined non-proportionality and damping indexes. The results present good agreement between the exact solution VEM and the EVM solution in terms of the frequency response function, even for non-proportional and relatively high damped systems. The advantages of the theory of linear viscously damped systems may be used now to obtain the response of non-viscous structures.

\section{Appendix 6.A Proof of the Lemma 1}

The norm of the vector $\delta \mathbf{u}_{j}$ can be bounded in terms of the matrix A. Actually, from the definition given in Eq. (6.36)

$$
\left\|\delta \mathbf{u}_{j}\right\|=\left\|\sum_{\substack{k=1 \\ k \neq j}}^{N} \alpha_{j k} \boldsymbol{\phi}_{k}\right\| \leq \sum_{\substack{k=1 \\ k \neq j}}^{N}\left|\alpha_{j k}\right|\left\|\boldsymbol{\phi}_{k}\right\| \leq\left(\sum_{k=1}^{N}\left|A_{j k}\right|\right)\left(\sum_{k=1}^{N}\left\|\boldsymbol{\phi}_{k}\right\|\right)=\left\|\mathbf{A}_{j}\right\| \cdot\|\mathbf{\Phi}\|
$$

where $\mathbf{A}_{j}$ is the $j$ th row of the matrix $\mathbf{A}$. Now, adding in $j=1, \ldots, N$, it follows

$$
\sum_{j=1}^{N}\left\|\delta \mathbf{u}_{j}\right\| \leq\left(\sum_{j=1}^{N}\left\|\mathbf{A}_{j}\right\|\right)\|\boldsymbol{\Phi}\|=\alpha\|\boldsymbol{\Phi}\|
$$

\section{Appendix 6.B Proof of the Lemma 2}

Let $\mathbf{u}_{j}$ and $\mathbf{u}_{j}^{*}$ be the eigenvectors associated to the pair of complex conjugate eigenvalues $s_{j}$ and $s_{j}^{*}$ respectively. Using the expansion introduced in subsection 6.3.2, the eigenrelations can be expressed as

$$
\begin{aligned}
{\left[s_{j}^{2} \mathbf{M}+s_{j} \mathbf{G}\left(s_{j}\right)+\mathbf{K}\right]\left(\boldsymbol{\phi}_{j}+\delta \mathbf{u}_{j}\right) } & =\mathbf{0} \\
{\left[s_{j}^{*^{2}} \mathbf{M}+s_{j}^{*} \mathbf{G}\left(s_{j}^{*}\right)+\mathbf{K}\right]\left(\boldsymbol{\phi}_{j}+\delta \mathbf{u}_{j}^{*}\right) } & =\mathbf{0}
\end{aligned}
$$

Now, pre-multiplying the previous equations by $\phi_{j}^{T}$ and using the modal identities

$$
\begin{aligned}
s_{j}^{2}+s_{j} \boldsymbol{\phi}_{j}^{T} \mathbf{G}\left(s_{j}\right)\left(\boldsymbol{\phi}_{j}+\delta \mathbf{u}_{j}\right)+\omega_{j}^{2} & =0 \\
s_{j}^{*^{2}}+s_{j}^{*} \boldsymbol{\phi}_{j}^{T} \mathbf{G}\left(s_{j}^{*}\right)\left(\boldsymbol{\phi}_{j}+\delta \mathbf{u}_{j}^{*}\right)+\omega_{j}^{2} & =0
\end{aligned}
$$

Subtracting both equations

$$
\left(s_{j}-s_{j}^{*}\right)\left(s_{j}+s_{j}^{*}\right)+s_{j} \boldsymbol{\phi}_{j}^{T} \mathbf{G}\left(s_{j}\right)\left(\boldsymbol{\phi}_{j}+\delta \mathbf{u}_{j}\right)-s_{j}^{*} \boldsymbol{\phi}_{j}^{T} \mathbf{G}\left(s_{j}^{*}\right)\left(\boldsymbol{\phi}_{j}+\delta \mathbf{u}_{j}^{*}\right)=0
$$


from here, the sum $s_{j}+s_{j}^{*}$ can already be read off, resulting in

$$
s_{j}+s_{j}^{*}=\gamma_{e j}\left[-s_{j} \boldsymbol{\phi}_{j}^{T} \mathbf{G}\left(s_{j}\right)\left(\boldsymbol{\phi}_{j}+\delta \mathbf{u}_{j}\right)++s_{j}^{*} \boldsymbol{\phi}_{j}^{T} \mathbf{G}\left(s_{j}^{*}\right)\left(\boldsymbol{\phi}_{j}+\delta \mathbf{u}_{j}^{*}\right)\right]
$$

Taking the absolute value and adding in $1 \leq j \leq N$, the bound can be evaluated as

$$
\begin{aligned}
\sum_{j=1}^{N}\left|s_{j}+s_{j}^{*}\right| & \leq \gamma[\Omega\|\mathbf{\Phi}\| \beta \Omega\|\mathbf{M}\|(1+\alpha)\|\mathbf{\Phi}\|++\Omega\|\mathbf{\Phi}\| \beta \Omega\|\mathbf{M}\|(1+\alpha)\|\mathbf{\Phi}\|] \\
& =2 \gamma \Omega^{2}\|\mathbf{\Phi}\|^{2}\|\mathbf{M}\|(1+\alpha) \beta
\end{aligned}
$$

where it the following inequalities have been taken into account

$$
\sum_{j=1}^{N}\left\|\phi_{j}+\delta \mathbf{u}_{j}\right\| \leq(1+\alpha)\|\mathbf{\Phi}\| \quad, \quad \sum_{j=1}^{N}\left\|\mathbf{G}\left(s_{j}\right)\right\| \leq \beta \Omega\|\mathbf{M}\|
$$

\section{Appendix 6.C Proof of the Lemma 3}

The complete expression of $\epsilon_{j}$ can be deduced taking absolute values of Eq. (6.46), adding in $1 \leq j \leq N$, resulting in

$$
\begin{aligned}
\sum_{j=1}^{N}\left|\epsilon_{j}\right| & \left.=\sum_{j=1}^{N}\left|\gamma_{e j}\right| \mid s_{j}+s_{j}^{*}+2 s_{j} \sum_{\substack{k=1 \\
k \neq j}}^{N} \alpha_{j k}^{2}++\left(\boldsymbol{\phi}_{j}^{T}+2 \delta \mathbf{u}_{j}^{T}\right) \overline{\mathbf{G}}\left(s_{j}\right) \boldsymbol{\phi}_{j}+\delta \mathbf{u}_{j}^{T} \overline{\mathbf{G}}\left(s_{j}\right) \delta \mathbf{u}_{j}\right] \\
& \leq \gamma\left[\sum_{j=1}^{N}\left|s_{j}+s_{j}^{*}\right|+2 \Omega \alpha^{2}(1+2 \alpha) \beta \Omega\|\mathbf{\Phi}\|^{2}\|\mathbf{M}\|+\alpha^{2} \beta \Omega\|\mathbf{\Phi}\|^{2}\|\mathbf{M}\|\right](6.100)
\end{aligned}
$$

Using now the Lemma 2 result and the inequality $N \leq\|\mathbf{\Phi}\|^{2}\|\mathbf{M}\|$, it follows that

$$
\begin{aligned}
\sum_{j=1}^{N}\left|\epsilon_{j}\right| & \leq \gamma\left[2 \gamma \Omega^{2}\|\mathbf{\Phi}\|^{2}\|\mathbf{M}\|(1+\alpha) \beta+\frac{2 N \Omega \alpha^{2}}{N}+(1+\alpha)^{2} \beta \Omega\|\mathbf{\Phi}\|^{2}\|\mathbf{M}\|\right] \\
& \leq\|\mathbf{\Phi}\|^{2}\|\mathbf{M}\| \gamma \Omega\left[\frac{2 \alpha^{2}}{N}+\beta(1+\alpha)(1+\alpha+2 \gamma \Omega)\right]
\end{aligned}
$$




\section{Appendix 6.D Proof of the Lemma 4}

Since $s_{j}$ is an eigenvalue

$$
\operatorname{det}\left[\boldsymbol{\Phi}^{T} \mathbf{D}\left(s_{j}\right) \boldsymbol{\Phi}\right]=\operatorname{det}\left[s_{j}^{2} \mathbf{I}_{N}+s_{j} \mathbf{G}^{\prime}\left(s_{j}\right)+\boldsymbol{\Lambda}\right]=0
$$

However, knowing that $\left\{p_{1}, \cdots, p_{h}\right\}$ are the poles of the function $\mathbf{G}(s)$, this determinant can be writen in the following form

$$
\prod_{k=1}^{N}\left(s_{j}^{2}+\omega_{k}^{2}\right)+\frac{\Gamma\left(s_{j}, \beta\right)}{\left(s_{j}-p_{1}\right)^{r_{1}} \cdots\left(s_{j}-p_{h}\right)^{r_{h}}}=0
$$

where $r_{l}, 1 \leq l \leq h$ is the multiplicity of the pole $p_{l}$, and $\Gamma\left(s_{j}, \beta\right)$ is a function that, although not depending directly on $\beta$, verifies $\lim _{\beta \rightarrow 0} \Gamma\left(s_{j}, \beta\right)=0$. The equality to zero is due to the product of matrix elements $\mathbf{G}^{\prime}\left(s_{j}\right)$, that tend to zero when $\beta \rightarrow 0$. Now, multiplying Eq. (6.102) by $\prod_{l=1}^{h}\left(s_{j}-p_{l}\right)$

$$
\prod_{l=1}^{h}\left(s_{j}-p_{l}\right)^{r_{l}} \prod_{k=1}^{N}\left(s_{j}^{2}+\omega_{k}^{2}\right)+\Gamma\left(s_{j}, \beta\right)=0
$$

If in the previous equation limits are taken when $\beta \rightarrow 0$, naming $\sigma_{j}=\lim _{\beta \rightarrow 0} s_{j}$ the expression becomes

$$
\prod_{l=1}^{h}\left(\sigma_{j}-p_{l}\right)^{r_{l}} \prod_{k=1}^{N}\left(\sigma_{j}^{2}+\omega_{k}^{2}\right)=0
$$

But, $\sigma_{j}^{2}+\omega_{k}^{2} \neq 0, \forall 1 \leq k \leq N$, due to $\sigma_{j} \in \mathbb{R}, \forall \beta>0$. Therefore, it is immediate that $\sigma_{j}$ belongs to the pole set, otherwise the equation would not vanish. 



\section{Bibliography}

[10] W. Flugge. Viscoelasticity. 2nd Edition. Springer-Verlag, 1975 (cit. on pp. 3, 160).

[11] A.D. Nashif, D.I.G. Jones, and J.P. Henderson. Vibration Damping. John Wiley and Sons, 1985 (cit. on pp. 3, 45, 160, 192, 194, 206).

[12] David I.G. Jones. Handbook of viscoelastic vibration damping. John Wiley \& Sons, 2001 (cit. on pp. 3, 45, 160, 192, 194).

[14] D.F. Golla and P.C. Hughes. "Dynamics of Viscoelastic Structures - A Time-domain, Finite-element Formulation." In: Journal of Applied MechanicsTransactions of the ASME 52.4 (1985), 897-906 (cit. on pp. 3, 20, 22, 24, $58,60,82,84,118,160,162,164,192)$.

[28] S. Adhikari. "Dynamics of Non-viscously Damped Linear Systems." In: Journal of Engineering Mechanics 128.3 (2002), 328-339 (cit. on pp. 4-6, $22,35,36,58,84,94,121,125,129,131,160-162,165,168,170,171,203)$.

[30] J. Woodhouse. "Linear Damping Models For Structural Vibration." In: Journal of Sound and Vibration 215.3 (1998), 547-569 (cit. on pp. 5, 35, $58,121,170,171)$.

[32] Sondipon Adhikari and Blanca Pascual. "Iterative Methods for Eigenvalues of Viscoelastic Systems." In: Journal of Vibration and Acoustics 133.2 (2011), pp. 021002.1-021002.7 (cit. on pp. 5, 21, 35, 42, 59, 83, 108, 119, $171,193)$.

[40] D.J. McTavish and P.C. Hughes. "Modeling of Linear Viscoelastic Space Structures." In: Journal of Vibration and Acoustics-Transactions of the ASME 115.1 (1993), 103-110 (cit. on pp. 20, 24, 58, 82, 118, 160, 164).

[54] A Muravyov and SG Hutton. "Closed-form solutions and the eigenvalue problem for vibration of discrete viscoelastic systems." In: Journal of Applied Mechanics-Transactions of the ASME 64.3 (1997), 684-691 (cit. on pp. 21, 58, 83, 119, 160, 164, 193).

[55] A. Muravyov. "Forced vibration responses of viscoelastic structure." In: Journal of Sound and Vibration 218.5 (1998), 892-907 (cit. on pp. 21, 38, $83,119,160)$. 
[56] S. Menon and J. Tang. "A state-space approach for the dynamic analysis of viscoelastic systems." In: Computers \& Structures 82.15-16 (2004), pp. 1123 -1130 (cit. on pp. 21, 83, 119, 160, 164, 193).

[64] P Muller. "Are the eigensolutions of a l-d.o.f. system with viscoelastic damping oscillatory or not?" In: Journal of Sound and Vibration 285.12 (2005), 501-509 (cit. on pp. 22, 38, 58, 84, 121, 162).

[73] A Muravyov and SG Hutton. "Free vibration response characteristics of a simple elasto-hereditary system." In: Journal of Vibration and AcousticsTransactions of the ASME 120.2 (1998), 628-632 (cit. on pp. 38, 58, 160, 162).

[94] A. Muravyov. "Analytical solutions in the time domain for vibration problems of discrete viscoelastic systems." In: Journal of Sound and Vibration 199.2 (1997), 337-348 (cit. on p. 160).

[95] J. P. Bandstra. "Comparison Of Equivalent Viscous Damping and Nonlinear Damping in Discrete and Continuous Vibrating Systems." In: Journal of Vibration, Acoustics, Stress and Reliability in Design 105 (1983), 382392 (cit. on pp. 160, 193).

[96] H. T. Banks and D. J. Inman. "On Damping Mechanisms in Beams." In: Journal of Applied Mechanics-Transactions of The ASME 58 (1991), 716723 (cit. on p. 161).

[97] A.M.G. de Lima, D.A. Rade, and F.P. Lépore Neto. "An efficient modeling methodology of structural systems containing viscoelastic dampers based on frequency response function substructuring." In: Mechanical Systems and Signal Processing 23.4 (2009), pp. 1272 -1281 (cit. on p. 161).

[98] Eric E. Ungar and Jr. Edward M. Kerwin. "Loss Factors of Viscoelastic Systems in Terms of Energy Concepts." In: The Journal of the Acoustical Society of America 34.7 (1962), pp. 954-957 (cit. on pp. 161, 193).

[99] C.D. Johnson and D.A. Kienholz. "Finite Element Prediction of Damping in Structures with Constrained Viscoelastic Layers." In: AIAA journal 20.9 (1982), pp. 1284-1290 (cit. on p. 161).

[100] A. Bilbao et al. "Proportional damping approximation for structures with added viscoelastic dampers." In: Finite Elements in Analysis and Design 42.6 (2006), pp. 492-502 (cit. on p. 161).

[101] G. Genta and N. Amati. "Hysteretic damping in rotordynamics: An equivalent formulation." In: Journal of Sound and Vibration 329.22 (2010), pp. 4772-4784 (cit. on p. 161).

[102] Nicos Makris and M.C. Constantinou. "Fractional-derivative Maxwell model for viscous dampers." In: Journal of structural engineering New York, N.Y. 117.9 (1991), pp. 2708-2724 (cit. on p. 161). 
[103] D.J. Segalman. "Calculation of Damping Matrices for Linearly Viscoelastic Structures." In: Journal of Applied Mechanics 54.3 (1987), 585-588 (cit. on pp. 161, 193).

[104] Sondipon Adhikari. "A Reduced Second-Order Approach for Linear Viscoelastic Oscillators." In: Journal of Applied Mechanics-Transactions of the ASME 77.4 (2010), pp. 1-8 (cit. on pp. 161, 193).

[105] S. Adhikari. "Optimal complex modes and an index of damping non-proportionality." In: Mechanical Systems and Signal Processing 18 (2004), 1-27 (cit. on p. 171). 



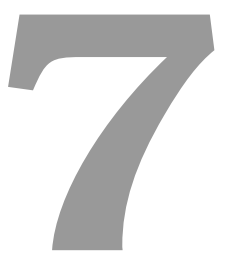

\section{Dynamic analysis of frame structures with free viscoelastic layers: New closed-form solutions of eigenvalues and a viscous approach}

\subsection{Introduction}

The use of viscoelastic materials for civil, mechanical and aeronautical engineering applications has expanded greatly in the last years. The publishing of better models of constitutive behavior, the development of faster computers and the emergence of new and efficient numerical algorithms has allowed a more accurately characterization of these materials and the successful implementation of complex models. Among the numerous applications of such materials we can highlight thin layers bounded to rigid structures to control and damp vibration. Two variants of this application exist: Firstly, the constrained viscoelastic layers located between two layers of elastic material, usually metal; the viscoelastic material dissipates energy mainly working under shear stress. Second, unconstrained free viscoelas- 
tic layers (FVL), bounded directly onto the elastic material and located in areas where normal stresses are prevalent. The latter solution is analyzed in this article and applied to framed structures.

The inclusion of viscoelastic materials in dynamic damping problems requires solving a system of integral-differential equations in which dissipative forces appear. These forces depend on the velocity history through certain kernel hereditary functions. Furthermore, in the frequency domain, the free motion equations transform into a nonlinear eigenvalue problem. The resolution of viscoelastic systems, both in the time and in the frequency domains, involves the introduction of new internal variables that increase the computational solution cost and the storage requirements. The challenge is to find equivalent numerical models, simpler than the original one, that attempt to reproduce the viscoelastic models' response. In this way, tools of proven efficiency available to solve dynamic problems with viscous damping can be used to solve other cases of higher computational complexity.

The constitutive models of viscoelastic materials are characterized by the variability in the frequency domain of their complex modulus. Among the many models available in the literature (see for example a review from Vasques [106]), two major groups can be mentioned. First, those in which hereditary functions are exponential kernels, derived from Biot's theory [7] and second, those which constitutive equations are governed by the fractional derivative, from the theory of Bagley and Torvik [15, 16]. This article will use the latter, in particular the called four-parameter model studied by Pritz [20]. More advanced ones, such as the five-parameter model [23], include an additional parameter allowing to extend the range of simulated materials at the expense of higher computational cost. From a physical point of view, the general criteria that damping functions must verify in structural dynamics are defined by Golla and Hughes [14].

The works of Nashif [11], Sun and Lu [13], and Jones [12] are of great importance in the field of engineering viscoelastic materials' applications for damping and vibration control of structures. In them, the most relevant theories of dynamic damping applied to structures and machinery are analyzed. Oberst and Frankenfeld [92] studied the problem of a beam that included FVL's, using the Euler-Bernoulli beam hypotheses under the assumption of small thickness. Cortés and Elejabarrieta [107] developed another method for dynamic analysis in time domain of beams with FVL, in which a specific model allowed to solve an auxiliary viscous response at each instant. The same authors in [107] extended the formulation to thick beams, without neglecting the shear deformation. Torvik [108] analyzed in a simplified way beams with symmetric and antisymmetric configurations of free viscoelastic coatings. Xu and Nashif [109] compared the experimental results of different specimens used to find the dynamic properties of viscoelastic 
materials using the standard ASTM-E-756, [110]. Rao [111] reviewed the main applications of damping techniques in the automotive and aerospace fields. One of the newest applications in civil engineering is the use of viscoelastic dampers for the control of seismic forces. See for details references [112-118] and in particular their application for the rehabilitation of existing railway bridges in [119].

From the development of advanced damping mechanisms, new viscous models able to predict their response through equivalent parameters have been sought. Bandstra [95] found equivalent viscosity coefficients for different nonlinear systems with a single degree-of-freedom (dof). Lee [120] calculated equivalent ratios for structures with added dampers. The Modal Strain Energy method was proposed by Ungar and Kerwin [98] to obtain the overall ratio in a viscoelastic system. Segalman [103] analyzed the viscoelasticity problem as the perturbation of a viscous problem, from a theoretical point of view under the assumption of low viscoelasticity and deducing expressions for the resulting second order system. Also under this hypotheses, although in the frequency domain Adhikari [104] proposed a second order and equivalent model evaluating the damping function at the complex eigenvalues. Lázaro and Pérez-Aparicio [121] have recently obtained a viscous model from the expansion of the viscoelastic transfer function, based on the availability of the undamped modal space and the solution of the nonlinear eigenvalue problem.

The numerical calculation of the complex eigenvalues is important for the modal analysis of viscoelastic structures. In systems in which the damping is governed by a rational function, the nonlinear eigenvalue problem can be transformed into a linear one but with a higher number of dof's. The increase of the matrices' size is directly proportional to the number of exponential kernels present in the damping matrix. This procedure has been developed in the works of Muravyov [54] and Menon [56]. For cases in which the model includes fractional derivatives, the transformation to augmented linear systems is also possible, provided that the fractional exponent is a rational number $[16,18]$. These methods have the shortcoming of producing again a larger model size and consequently storage. In this sense, one can apply the general methods used for nonlinear eigenvalue problems based on an iterative approach to the solution through the first order expansion of the transcendent matrix; for more details see the references [46, 93]. From a computational standpoint, systems in which the damping matrix is proportional or slightly non-proportional are very advantageous. Adhikari and Pascual [31] obtained approximated expressions for the calculation of real and complex eigenvalues using the first iteration of Newton's scheme, and later extended this scheme with an iterative method [32]. Lázaro and Pérez-Aparicio [33] have recently developed a highly efficient method based on the construction of two functions that recursively operate in the complex plane. The same authors in [63], considering the eigenvalues as functions of a certain parameter, have proposed another method 
for single dof systems. The extension to a multidimensional parameter and to systems of multiple dof's can be found in [34].

This paper develops a new method for the analysis of two-dimensional structures with FVL's based on fractional derivatives using the equivalent viscous model proposed by the authors in [121]. To this end, it is necessary to calculate the complex eigenvalues' set. The main contribution of the current paper is the development of closed-form expressions for the eigenvalues in terms of damping parameters; the methodology allow us to directly construct the equivalent viscous model without adding auxiliary variables or using iterative methods. In this way, we explicitly obtain new expressions of the equivalent stiffness and viscous damping matrices, also function of the viscoelastic damping parameters. Finally, the proposed method is validated by two numerical examples; the first for a cantilever beam and the second for a frame, both with added viscoelastic layers. The eigenvalue and transfer function errors are analyzed for a wide range of damping materials and related parameters.

\subsection{Finite element formulation in frequency domain}

As mentioned, the structures covered by this article are lattice planes of beams with FVL bonded to a metallic base material. To obtain the corresponding equations of motion, the assumptions from articles [11-13, 92] are commonly adopted:

- The viscoelastic layers are fully bonded to the surface of the base material and arranged in its top and/or bottom

- The thickness of the viscoelastic layer is small compared with the section depth, considered constant

- Classic flexural behavior of slender beams: Hypotheses of Euler-Bernoulli

- Linear elastic behavior without damping for the base material

— Viscoelastic behavior of the bonded layers based on the fractional derivative

— The stiffness of the base material is much larger than that of the FVL

All structural systems containing viscoelastic materials can be characterized by a damping matrix, function of frequency. To obtain the equations of motion we will use the principle of virtual work in the frequency domain, as it is usual in spectral analysis [122, 123]. 

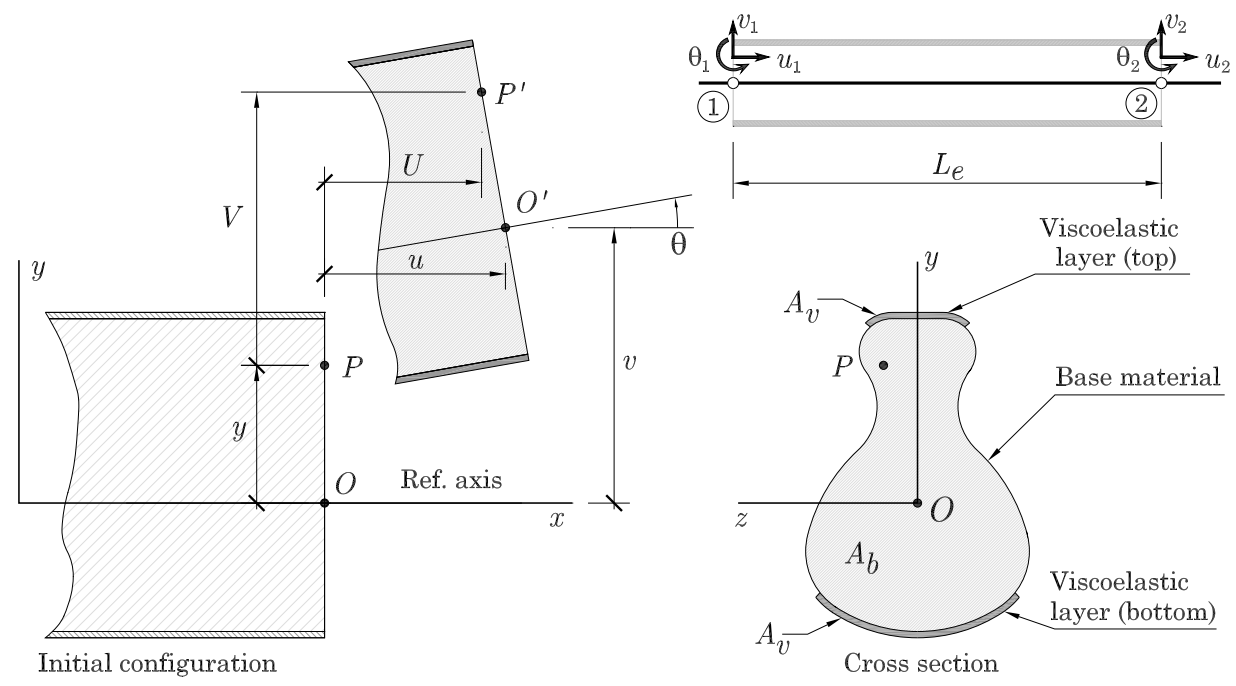

Figure 7.1: Displacement of a beam cross section generic points and of the reference point. Structural configuration of the viscoelastic layer.

\subsubsection{Kinematic and constitutive relationships}

In Fig. 7.1, the kinematics of a beam section $x$ are represented; a reference system $\{x, y, z\}$ is centered at a generic point $O$. Let $\mathbf{d}(x, y, t)=\{U, V\}^{T}$ be the displacement vector from any point $P$ to other position $P^{\prime}$. This point can belong to the base or to the viscoelastic materials. Due to the hypothesis of plane sections remaining plane, the vector does not depend on the coordinate $z$. Let finally $\mathbf{v}(x, t)=\{u, v, \theta\}^{T}$ be the displacement and rotation vector of $O$ with respect to the reference axes.

Consider now a Finite Element (FE) of length $L_{e}$ with two nodes which dof's are arranged in the column vector $\mathbf{u}_{e}(t)=\left\{u_{1}, v_{1}, \theta_{1}, u_{2}, v_{2}, \theta_{2}\right\}^{T}$. As a result of the FE discretization, the displacements and rotations are interpolated so that

$$
\mathbf{v}(x, t) \approx \mathbf{N}(x) \mathbf{u}_{e}(t)
$$

where $\mathbf{N}(x)$ is the matrix that contains the shape functions. The vector can be written as a product of separate functions

$\mathbf{d}(x, y, t)=\left\{\begin{array}{l}U \\ V\end{array}\right\}=\left[\begin{array}{ccc}1 & 0 & -y \\ 0 & 1 & 0\end{array}\right]\left\{\begin{array}{l}u \\ v \\ \theta\end{array}\right\} \equiv \mathbf{B}_{1}(y) \mathbf{v}(x, t) \approx \mathbf{B}_{1}(y) \mathbf{N}(x) \mathbf{u}_{e}(t)$ 
and the longitudinal strain $\epsilon_{x}$ is expressed as

$$
\epsilon_{x}=\frac{\partial U}{\partial x}=\left[\begin{array}{ll}
1 & 0
\end{array}\right] \frac{\partial \mathbf{d}}{\partial x} \equiv \mathbf{B}_{2}(y) \frac{\partial \mathbf{d}}{\partial x}=\mathbf{B}_{2}(y) \mathbf{B}_{1}(y) \frac{\partial \mathbf{N}}{\partial x} \mathbf{u}_{e}(t) \equiv \mathbf{B}(y) \frac{\partial \mathbf{N}}{\partial x} \mathbf{u}_{e}(t)
$$

As mentioned, the general form of models based on the fractional derivative was introduced by Bagley and Torvick [16] and consists on a linear and fractional differential equation that relates stresses and strains. The same authors suggested in [19] a simpler relation, with relatively few terms which permit the description of a wide range of materials. They also demonstrated that the order of the fractional derivatives of stresses must be the same as that of strains to satisfy thermodynamic considerations; hence the model is usually named four-parameter model.

$$
\sigma_{x}+\tau^{\alpha} \frac{\mathrm{d}^{\alpha} \sigma_{x}}{\mathrm{~d} t^{\alpha}}=E_{0} \epsilon_{x}+E_{\infty} \tau^{\alpha} \frac{\mathrm{d}^{\alpha} \epsilon_{x}}{\mathrm{~d} t^{\alpha}}
$$

where $\mathrm{d}^{\alpha} / \mathrm{d} t^{\alpha}$ represents the fractional derivative of order $\alpha$, often assuming $0<$ $\alpha<1$, see [15]. In Eq. (7.4), $\tau>0$ is the relaxation time and $E_{0}$ the static Young modulus, that is, the dynamic modulus at zero frequency $\omega \rightarrow 0$. $E_{\infty}$ is the high frequency limit value of the dynamic modulus $\omega \rightarrow \infty$, also named storage modulus because $E_{\infty}>E_{0}$. It is common to introduce the non-dimensional parameter $c=E_{\infty} / E_{0}>1$. Pritz [20] studied the four-parameter model in the frequency domain describing the influence of $\tau, \alpha$ and $c$ for a large variety of practical materials. Eq. (7.4) can be transformed to the frequency domain

$$
\begin{aligned}
\hat{\sigma}_{x}(i \omega) & =\frac{E_{0}+E_{\infty}(i \omega \tau)^{\alpha}}{1+(i \omega \tau)^{\alpha}} \hat{\epsilon}_{x}(i \omega) \\
& =E_{0} \frac{1+c(i \omega \tau)^{\alpha}}{1+(i \omega \tau)^{\alpha}} \hat{\epsilon}_{x}(i \omega) \equiv \hat{E}_{v}(i \omega) \hat{\epsilon}_{x}(i \omega)
\end{aligned}
$$

where $\hat{\sigma}_{x}(i \omega)=\mathcal{F}\left\{\sigma_{x}(t)\right\}$ and $\hat{\epsilon}_{x}(i \omega)=\mathcal{F}\left\{\epsilon_{x}(t)\right\}$ are the Fourier transforms of stress and strain, $i=\sqrt{-1}$ the imaginary unit and

$$
\hat{E}_{v}(i \omega)=E_{d}(\omega)+i E_{l}(\omega) \equiv E_{d}(\omega)[1+i \eta(\omega)]
$$

the complex modulus which real and imaginary parts provide relevant information about the viscoelasticity induced in the structure; $E_{d}(\omega)$ and $E_{l}(\omega)$ are the dynamic and loss moduli, respectively. The frequency dependence of $\hat{E}_{v}(i \omega)$ is named dispersion. A large variation is associated with high viscoelasticity, the contrary is found in low viscoelasticity. The function $\eta(\omega)=E_{l} / E_{d}$ is the loss factor of the material and may be used to measure the system viscoelasticity level [124]. Pritz [20, 22] studied loss factors for real solids finding two interesting properties: First, for most materials the curve $\eta(\omega)$ presents a single peak. Second, the loss factor peak $\eta_{m}$ is a good indicator for the quantification of viscoelasticity in the damping model and its expression is

$$
\eta_{m}=\frac{(c-1) \sin (\alpha \pi / 2)}{2 \sqrt{c}+(c+1) \cos (\alpha \pi / 2)}
$$


According to Pritz, low values $10^{-3} \leq \eta_{m} \leq 10^{-1}$ are characteristic of hard plastics and other structural materials with weak frequency dependence of the dynamic modulus, such as wood, concrete, metals, etc. On the other hand, large values $10^{-1} \leq \eta_{m} \leq 1$ are characteristic of rubbers and rubber-like materials used for vibration isolation and damping. The factor $\eta_{m}$ will be used later in the numerical examples due to its relevance in the results. Other indicators for viscoelasticity quantification are available in the work of Adhikari \& Woodhouse [71].

All viscoelastic materials are characterized by dissipative forces that depend on the history of the response. Therefore, a kernel hereditary function $\mathcal{G}(t)$ is defined so that the constitutive relationship in the time domain Eq. (7.4) can analitically be expressed as

$$
\sigma_{x}(t)=E_{0} \epsilon_{x}(t)+\int_{-\infty}^{t} \mathcal{G}(t-\tau) \dot{\epsilon}_{x}(\tau) \mathrm{d} \tau
$$

Calculating the Fourier transform of the above expression

$$
\hat{\sigma}_{x}(i \omega)=\left[E_{0}+i \omega G(i \omega)\right] \hat{\epsilon}_{x}(i \omega)
$$

where $G(i \omega)=\mathcal{F}\{\mathcal{G}(t)\}$ is the damping function in the frequency domain. For constitutive models based on the fractional derivative $\mathcal{G}(t)$ is not explicitly known. However, $G(i \omega)$ can be deduced equating the above expression to the constitutive Eq. (7.5)

$$
G(i \omega)=E_{0} \frac{c-1}{i \omega} \frac{(i \omega \tau)^{\alpha}}{1+(i \omega \tau)^{\alpha}} \equiv E_{0} \psi(i \omega)
$$

In the following, the base material (the beam) will be considered metallic and a linear elastic behavior with elastic modulus $E_{b}$ assumed for it.

\subsubsection{Motion equation in frequency domain}

The basic developments of this article are developed in the frequency domain, therefore it is necessary to derive the motion equations in this domain. To this end, it is useful to express the principle of virtual work in the frequency domain as in $[122,123]$, where spectral element analyses were developed. The virtual work of inertial and elastic forces must be equal to the virtual work of external forces

$$
-\omega^{2} \sum_{e} \int_{\mathcal{V}^{(e)}} \delta \hat{\mathbf{d}}^{T} \rho \hat{\mathbf{d}} \mathrm{d} V+\sum_{e} \int_{\mathcal{V}^{(e)}} \delta \hat{\epsilon}_{x}^{T} \hat{\sigma}_{x} \mathrm{~d} V=\sum_{e} \int_{0}^{L_{e}} \delta \hat{\mathbf{v}}^{T} \hat{\mathbf{f}} \mathrm{d} x
$$

where $\rho$ is the density of either the base or viscoelastic material, $\hat{\mathbf{f}}$ the Fourier transform of the vector of distributed forces reduced to the reference axis and $\mathcal{V}^{(e)}=L_{e} \times \mathcal{A}$ the finite element volume of section $\mathcal{A}=\mathcal{A}_{b} \cup \mathcal{A}_{v}$, where $\mathcal{A}_{b}$ and $\mathcal{A}_{v}$ are the total areas of the base and viscoelastic materials, respectively, see Fig. 7.1. 
The summation with element number $e$ index refers to assembly in the standard finite element context.

Using Eq. (7.2) and separating the integrals according to the materials' area, we obtain

$$
\begin{aligned}
& -\omega^{2} \int_{\mathcal{V}(e)} \delta \hat{\mathbf{d}}^{T} \rho \hat{\mathbf{d}} \mathrm{d} V \\
= & -\omega^{2} \delta \hat{\mathbf{u}}_{e}^{T} \int_{0}^{L_{e}} \mathbf{N}^{T}(x)\left[\int_{\mathcal{A}} \mathbf{B}_{1}^{T}(y) \rho \mathbf{B}_{1}(y) \mathrm{d} y \mathrm{~d} z\right] \mathbf{N}(x) \mathrm{d} x \hat{\mathbf{u}}_{e} \\
= & -\omega^{2} \delta \hat{\mathbf{u}}_{e}^{T} \int_{0}^{L_{e}} \mathbf{N}^{T}(x)\left(\mathbf{m}_{b}+\mathbf{m}_{v}\right) \mathbf{N}(x) \mathrm{d} x \hat{\mathbf{u}}_{e} \\
\equiv & -\omega^{2} \delta \hat{\mathbf{u}}_{e}^{T}\left(\mathbf{M}_{b}^{(e)}+\mathbf{M}_{v}^{(e)}\right) \hat{\mathbf{u}}_{e} \\
\equiv & -\omega^{2} \delta \hat{\mathbf{u}}_{e}^{T} \mathbf{M}^{(e)} \hat{\mathbf{u}}_{e}
\end{aligned}
$$

where

$$
\begin{aligned}
\mathbf{m}_{b} & =\int_{\mathcal{A}_{b}} \mathbf{B}_{1}^{T}(y) \rho_{b} \mathbf{B}_{1}(y) \mathrm{d} y \mathrm{~d} z=\rho_{b}\left[\begin{array}{ccc}
A_{b} & 0 & -S_{b} \\
0 & A_{b} & 0 \\
-S_{b} & 0 & I_{b}
\end{array}\right] \\
\mathbf{m}_{v} & =\int_{\mathcal{A}_{v}} \mathbf{B}_{1}^{T}(y) \rho_{v} \mathbf{B}_{1}(y) \mathrm{d} y \mathrm{~d} z=\rho_{v}\left[\begin{array}{ccc}
A_{v} & 0 & -S_{v} \\
0 & A_{v} & 0 \\
-S_{v} & 0 & I_{v}
\end{array}\right] \\
\mathbf{M}_{b}^{(e)}=\int_{0}^{L_{e}} \mathbf{N}^{T}(x) \mathbf{m}_{b} \mathbf{N}(x) \mathrm{d} x, \quad \mathbf{M}_{v}^{(e)} & =\int_{0}^{L_{e}} \mathbf{N}^{T}(x) \mathbf{m}_{v} \mathbf{N}(x) \mathrm{d} x
\end{aligned}
$$

In the previous matrices, $A_{b}, S_{b}, I_{b}$ denote the area, static moment and inertia moment of the cross section corresponding to the base material and calculated respect to the reference axes $\{O, x, y, z\}$, see Fig. 7.1. Analogously, $A_{v}, S_{v}, I_{v}$ denote those of the viscoelastic material. 
From Eqs. (7.3), (7.9), the virtual work of the internal elastic forces takes the expression

$$
\begin{aligned}
& \int_{\mathcal{V}(e)} \delta \hat{\epsilon}_{x}^{T} \hat{\sigma}_{x} \\
= & \delta \hat{\mathbf{u}}_{e}^{T} \int_{0}^{L_{e}} \frac{\partial \mathbf{N}^{T}}{\partial x}\left[\int_{\mathcal{A}_{b}} \mathbf{B}^{T}(y) E_{b} \mathbf{B}(y) \mathrm{d} y \mathrm{~d} z\right] \frac{\partial \mathbf{N}}{\partial x} \mathrm{~d} x \hat{\mathbf{u}}_{e} \\
+ & \delta \hat{\mathbf{u}}_{e}^{T} \int_{0}^{L_{e}} \frac{\partial \mathbf{N}^{T}}{\partial x}\left[\int_{\mathcal{A}_{v}} \mathbf{B}^{T}(y)\left[E_{0}+i \omega G(i \omega)\right] \mathbf{B}(y) \mathrm{d} y \mathrm{~d} z\right] \frac{\partial \mathbf{N}}{\partial x} \mathrm{~d} x \hat{\mathbf{u}}_{e} \\
= & \delta \hat{\mathbf{u}}_{e}^{T}\left(\int_{0}^{L_{e}} \frac{\partial \mathbf{N}^{T}}{\partial x}\left(\mathbf{D}_{b}+\mathbf{D}_{v}\right) \frac{\partial \mathbf{N}}{\partial x} \mathrm{~d} x+i \omega \frac{G(i \omega)}{E_{0}} \int_{0}^{L_{e}} \frac{\partial \mathbf{N}^{T}}{\partial x} \mathbf{D}_{v} \frac{\partial \mathbf{N}}{\partial x} \mathrm{~d} x\right) \hat{\mathbf{u}}_{e} \\
\equiv & \delta \hat{\mathbf{u}}_{e}^{T}\left(\mathbf{K}^{(e)}+i \omega \frac{G(i \omega)}{E_{0}} \mathbf{K}_{v}^{(e)}\right) \hat{\mathbf{u}}_{e} \equiv \delta \hat{\mathbf{u}}_{e}^{T}\left(\mathbf{K}^{(e)}+i \omega \mathbf{G}^{(e)}(i \omega)\right) \hat{\mathbf{u}}_{e} \quad(7.16)
\end{aligned}
$$

where

$$
\begin{aligned}
& \mathbf{D}_{b}=\int_{\mathcal{A}_{b}} \mathbf{B}^{T}(y) E_{b} \mathbf{B}(y) \mathrm{d} y \mathrm{~d} z=E_{b}\left[\begin{array}{ccc}
A_{b} & 0 & -S_{b} \\
0 & 0 & 0 \\
-S_{b} & 0 & I_{b}
\end{array}\right] \\
& \mathbf{D}_{v}=\int_{\mathcal{A}_{v}} \mathbf{B}^{T}(y) E_{0} \mathbf{B}(y) \mathrm{d} y \mathrm{~d} z=E_{0}\left[\begin{array}{ccc}
A_{v} & 0 & -S_{v} \\
0 & 0 & 0 \\
-S_{v} & 0 & I_{v}
\end{array}\right]
\end{aligned}
$$

The element stiffness and damping matrix finally are

$$
\mathbf{K}^{(e)}=\mathbf{K}_{b}^{(e)}+\mathbf{K}_{v}^{(e)}, \quad \mathbf{G}^{(e)}(i \omega)=\frac{G(i \omega)}{E_{0}} \mathbf{K}_{v}^{(e)}
$$

Introducing these matrices in Eq. (7.11) results in

$$
\delta \hat{\mathbf{u}}_{e}^{T}\left(-\omega^{2} \sum_{e} \mathbf{M}^{(e)}+i \omega \sum_{e} \mathbf{G}^{(e)}(i \omega)+\sum_{e} \mathbf{K}^{(e)}\right) \hat{\mathbf{u}}_{e}=\sum_{e} \delta \hat{\mathbf{u}}_{e}^{T} \int_{0}^{L_{e}} \mathbf{N}^{T} \hat{\mathbf{f}} \mathrm{d} x
$$

Naming

$$
\mathbf{M}=\sum_{e} \mathbf{M}^{(e)}, \mathbf{K}=\sum_{e} \mathbf{K}^{(e)}=\mathbf{K}_{b}+\mathbf{K}_{v}, \mathbf{G}(i \omega)=\sum_{e} \mathbf{G}^{(e)}(i \omega)=\frac{G(i \omega)}{E_{0}} \mathbf{K}_{v}
$$

to the assembled mass, stiffness and damping matrices,

$$
\hat{\mathbf{F}}=\sum_{e} \int_{0}^{L_{e}} \mathbf{N}^{T} \hat{\mathbf{f}} \mathrm{d} x
$$


to the nodal force vector and $\hat{\mathbf{u}}$ to the column vector that groups the complete set of active dof's, the motion equations in frequency domain can be written as

$$
\left(-\omega^{2} \mathbf{M}+i \omega \mathbf{G}(i \omega)+\mathbf{K}\right) \hat{\mathbf{u}}=\hat{\mathbf{F}}
$$

If the Fourier transform of the force vector is available, the response vector can be calculated by inversion of the combined stiffness matrix $\mathcal{K}(i \omega)$, leading to the definition of the receptance matrix

$$
\hat{\mathbf{u}}=\mathcal{K}^{-1}(i \omega) \hat{\mathbf{F}} \equiv \mathbf{H}(i \omega) \hat{\mathbf{F}}
$$

The receptance $\mathbf{H}(i \omega)$ contains all information needed to solve the dynamic problem in the frequency domain because its elements are the Frequency Response Functions (FRF) of the dof's. The dynamic matrices from Eq. (7.23) have been implemented in a Matlab ${ }^{(C)}$ [125] specific code for framed structures, taking into account the explicit forms of Eqs. (7.17), (7.18) and analytically developing the complete expressions.

\subsection{The viscous approach}

The viscous damping has traditionally been one of the most widely used tools to model energy dissipation in structural dynamics. When a structure contains viscoelastic materials viscous damping can not be used, it is necessary to apply advanced constitutive models as those from Eq. (7.4). The main disadvantage of these models is their complexity due to the introduction of new internal variables, and the mentioned increase in computer storage. The objective of the current work is to develop a computationally simple equivalent viscous model that can predict the structure response with sufficient approximation, with form

$$
\mathbf{M} \ddot{\mathbf{u}}+\mathbf{C}_{\mathrm{eq}}(\alpha, c, \tau) \dot{\mathbf{u}}+\mathbf{K}_{\mathrm{eq}}(\alpha, c, \tau) \mathbf{u}=\mathbf{F}(t)
$$

In the previous equation, $\mathbf{K}_{\mathrm{eq}}, \mathbf{C}_{\mathrm{eq}}$ are the stiffness and damping matrices that depend on the original dynamic arrays $\mathbf{K}, \mathbf{M}$ and on parameters $\alpha, c, \tau$ from a damping model based on fractional derivatives. In addition, $\mathbf{C}_{\mathrm{eq}}$ is proportional, i.e., is diagonalizable in the same modal basis as $\mathbf{M}, \mathbf{K}_{\mathrm{eq}}$. To obtain $\mathbf{K}_{\mathrm{eq}}$ and $\mathbf{C}_{\mathrm{eq}}$, information from the associated nonlinear eigenvalue problem will be developed in the following sections. 


\subsubsection{Closed-form expressions for eigenvalues}

The nonlinear eigenvalue expression is obtained introducing $\hat{\mathbf{F}} \equiv 0$ in Eq. (7.23). For convenience, the Laplace variable is used to represent frequency, $s=i \omega$

$$
\left(s^{2} \mathbf{M}+s \mathbf{G}(s)+\mathbf{K}\right) \hat{\mathbf{u}} \equiv \mathcal{K}(s) \hat{\mathbf{u}}=\mathbf{0}
$$

The solution of the previous problem is given by $N$ pairs of conjugated complex eigenvalues. In general, these eigenvalues may be obtained by any of the iterative numerical procedures available in the literature and described in the introduction. However, the challenge here is to find explicit analytic expressions for the eigenvalue calculation with a single evaluation and including all parameters. To this end, the parametric damping matrix method successfully applied by the authors in $[34,63]$, is used. In these references, the damping of the structure is based on the exponential model of Biot, assuming that the damping function is a multivariable function of $s$ and of the damping parameters

$$
\mathbf{G}(s, \alpha, c, \tau)=\frac{c-1}{s} \frac{(s \tau)^{\alpha}}{1+(s \tau)^{\alpha}} \mathbf{K}_{v} \equiv \psi(s, \alpha, c, \tau) \mathbf{K}_{v}
$$

Then, Eq. (7.26) implicitly defines the $2 N$ eigenvalues and $2 N$ eigenvectors as functions $\lambda_{j}=\lambda_{j}(\alpha, c, \tau), \mathbf{u}_{j}=\mathbf{u}_{j}(\alpha, c, \tau)$. Obviously, one can not find an exact analytical expression for $\lambda_{j}(\alpha, c, \tau)$, however, under certain assumptions good approximations can be developed. To do this, assume that each eigenvalue is a function only of the storage parameter $c$, so that the remaining $\tau, \alpha$ are held fixed. Thus, the $j$ th eigenvalue can be represented simply by $\lambda_{j}=\lambda_{j}(c)$, a function that can be approximated by the first order Taylor expansion in a neighborhood of an certain value $c_{0}$

$$
\lambda_{j}(c) \approx \lambda_{j}\left(c_{0}\right)+\frac{\partial \lambda_{j}\left(c_{0}\right)}{\partial c}\left(c-c_{0}\right) \equiv \lambda_{j, \operatorname{lin}}(c)
$$

The initial $c_{0}$ is not arbitrary, it is chosen to simultaneously verify that $\lambda_{j}\left(c_{0}\right)$ and $\partial \lambda_{j}\left(c_{0}\right) / \partial c$ are defined values that can explicitly be found as function of $\alpha, \tau$. Observing Eq. (7.27), it is clear that by choosing $c_{0}=1$ automatically $\lambda_{j}(1)=i \omega_{j}$ is verified. The frequency $\omega_{j}$ is the $j$ th natural frequency of the undamped linear problem

$$
\left(s^{2} \mathbf{M}+\mathbf{K}\right) \hat{\mathbf{u}}=\mathbf{0}
$$

For the computation of $\partial \lambda_{j}\left(c_{0}\right) / \partial c$, some additional steps are necessary. Under the previous considerations, the damping function can simply be expressed as a matrix function of two variables, $\mathbf{G}(s, c)$. Evaluating Eq. (7.26) with $s=\lambda_{j}(c)$, the associated eigenvalue $\hat{\mathbf{u}}=\mathbf{u}_{j}(c)$ is defined, so that

$$
\left[\lambda_{j}^{2}(c) \mathbf{M}+\lambda_{j}(c) \mathbf{G}\left(\lambda_{j}(c), c\right)+\mathbf{K}\right] \mathbf{u}_{j}(c)=\mathbf{0}
$$


The equation above can be written in the modal basis of the undamped problem; calling $\boldsymbol{\Phi}$ to the modal matrix which columns are the mass-normalized eigenvectors, the following well-known orthogonal relations are verified

$$
\boldsymbol{\Phi}^{T} \mathbf{M} \boldsymbol{\Phi}=\mathbf{I}_{N}, \quad \boldsymbol{\Phi}^{T} \mathbf{K} \boldsymbol{\Phi}=\boldsymbol{\Lambda}
$$

where $\mathbf{I}_{N}$ is the unit matrix of order $N$ and $\boldsymbol{\Lambda}=\operatorname{diag}\left[\omega_{j}^{2}\right]$. Performing the base change of the eigenvector $\mathbf{u}_{j}(c)=\mathbf{\Phi} \mathbf{q}_{j}(c)$ and premultipling by $\boldsymbol{\Phi}^{T}$

$$
\left[\lambda_{j}^{2}(c) \mathbf{I}_{N}+\lambda_{j}(c) \boldsymbol{\Gamma}\left(\lambda_{j}(c), c\right)+\boldsymbol{\Lambda}\right] \mathbf{q}_{j}(c)=\mathbf{0}
$$

In Eq. (7.32), $\boldsymbol{\Gamma}(s, p)=\boldsymbol{\Phi}^{T} \mathbf{G}(s, p) \boldsymbol{\Phi}$ is the damping matrix in the modal base. We assume that the structure composed of base and viscoelastic materials fulfills proportionality, that is, the damping matrix is diagonally dominant in the modal base. Thus, the determinant of the stiffness matrix from Eq. (7.32) can be decomposed into the product of its main diagonal entries

$$
\begin{aligned}
\operatorname{det}\left[\lambda_{j}^{2}(c) \mathbf{I}_{N}+\lambda_{j}(c) \boldsymbol{\Gamma}\left(\lambda_{j}(c), c\right)+\boldsymbol{\Lambda}\right] & \approx \prod_{j=1}^{N}\left[\lambda_{j}^{2}(c)+\lambda_{j}(c) \Gamma_{j j}\left(\lambda_{j}(c), c\right)+\omega_{j}^{2}\right] \\
& \equiv \prod_{j=1}^{N} D_{j}(c)=0
\end{aligned}
$$

The $j$ th diagonal entry of the damping matrix can be written in terms of the function $\psi(s, c)$ defined in Eq. (7.27). It is considered again that this function depends on the frequency $s$ and on the selected parameter $c$, leaving the rest fixed. Thus, substituting the expression of the damping matrix given by Eq. (7.27), $\Gamma_{j j}(s, c)=\psi(s, c) \kappa_{j}$, where by definition $\kappa_{j}=\boldsymbol{\phi}_{j}^{T} \mathbf{K}_{v} \boldsymbol{\phi}_{j}$. The equation that defines the $j$ th eigenvalue can now be written as

$$
D_{j}(c)=\lambda_{j}^{2}(c)+\lambda_{j}(c) \psi\left(\lambda_{j}(c), c\right) \kappa_{j}+\omega_{j}^{2}=0
$$

equality valid for any $c$, and hence its derivative is also zero

$$
\frac{\partial D_{j}}{\partial c}=2 \lambda_{j} \frac{\partial \lambda_{j}}{\partial c}+\left[\lambda_{j}\left(\partial_{s} \psi \frac{\partial \lambda_{j}}{\partial c}+\partial_{c} \psi\right)+\frac{\partial \lambda_{j}}{\partial c} \psi\left(\lambda_{j}, c\right)\right] \kappa_{j}=0
$$

where

$$
\left.\partial_{s} \psi \equiv \frac{\partial \psi}{\partial s}\right|_{s=\lambda_{j}},\left.\quad \partial_{c} \psi \equiv \frac{\partial \psi}{\partial c}\right|_{s=\lambda_{j}}
$$

Solving for the eigenvalue derivative, we have

$$
\frac{\partial \lambda_{j}}{\partial c}=-\lambda_{j} \frac{\partial_{c} \psi}{\lambda_{j}\left(2+\kappa_{j} \partial_{s} \psi\right)+\kappa_{j} \psi\left(\lambda_{j}, c\right)} \equiv-\lambda_{j} \mathcal{R}_{j}\left(\lambda_{j}, c\right)
$$


From the analytical expression of $\psi(s, c)$ given by Eq. (7.27), the derivatives $\partial_{s} \psi$ and $\partial_{c} \psi$ can be obtained and substituted in Eq. (7.37) to obtain $\mathcal{R}_{j}(s, c)$ as

$$
\mathcal{R}_{j}(s, c)=\frac{(s \tau)^{\alpha}\left[1+(s \tau)^{\alpha}\right]}{(c-1) \alpha \kappa_{j}(s \tau)^{\alpha}+2 s^{2}\left[1+(s \tau)^{\alpha}\right]^{2}}
$$

Since $\lambda_{j}(1)=i \omega_{j} \equiv \lambda_{j}^{0}$ is available from the solution of Eq. (7.29), we can evaluate Eq. (7.38) at $c=1$ to obtain $\partial \lambda_{j}(1) / \partial c$. Hence, the linear approximation $\lambda_{j, \operatorname{lin}}(c)$ can be finally obtained from Eq. (7.37) as

$$
\lambda_{j, \operatorname{lin}}(c)=i \omega_{j}-\frac{c-1}{2 i \omega_{j}} \frac{\left(i \omega_{j} \tau\right)^{\alpha}}{1+\left(i \omega_{j} \tau\right)^{\alpha}}
$$

Eq. (7.28) along with the initial value $\lambda_{j}^{0}=\lambda_{j}(1)$ defines an ordinary differential equation that can be solved with any of the available numerical methods (Euler, Runge-Kutta, ...). From the solution, $\lambda_{j}(c)$ can be numerically obtained. However, the physical characteristics of the problem allow us to assume certain hypothesis that result in an analytical and estimated solution of this differential equation, greatly reducing the computational cost of calculating the $j$ th eigenvalue. Indeed, it may be noted that $\mathcal{R}_{j}(s, c)$ depends both on $\psi(s, c)$ and on its derivatives. The variation of $\psi(s, c)$ in the frequency domain defines the viscoelasticity induced by the damping of the viscoelastic material. Mathematically, materials with high viscoelasticity present strong variations of $\psi(s, c)$; by contrast, a function with smooth and regular variations represents low viscoelasticity. More details on assessment and measurement of viscoelasticity can be found in references [28, 71]. For materials with small viscoelasticity, it is expected that when the following approximation is used

$$
\mathcal{R}_{j}\left(\lambda_{j}, c\right) \approx \mathcal{R}_{j}\left(\lambda_{j, \operatorname{lin}}(c), c\right)=\mathcal{R}_{j}\left(i \omega_{j}-\frac{c-1}{2 i \omega_{j}} \frac{\left(i \omega_{j} \tau\right)^{\alpha}}{1+\left(i \omega_{j} \tau\right)^{\alpha}}, c\right) \equiv R_{j}(c)
$$

the error is not very high. Thus, the problem of obtaining $\lambda_{j}(c)$ is reduced to solve the ordinary differential equation with separated variables

$$
\frac{\partial \lambda_{j}}{\partial c}=-\lambda_{j} R_{j}(c)
$$

In order to obtain a closed analytical integral expression, we expand $R_{j}(c)$ in Taylor series around point $c=1$ to directly calculate

$$
R_{j}(c)=\sum_{n=0}^{\infty} \frac{R_{j}^{(n)}(1)}{n !}(c-1)^{n}
$$

By successive derivation with respect to $c$ in Eq. (7.40), we find (see Appendix 7.A) that the $n$th coefficient is

$$
\frac{R_{j}^{(n)}(1)}{n !}=\left.\frac{1}{n !} \frac{\partial^{n} R_{j}}{\partial c^{n}}\right|_{c=1}=(-1)^{n+1}\left(\frac{\mu_{j}-1}{\mu_{j}}\right)^{n+1}\left(\frac{\kappa_{j}}{\omega_{j}^{2}}\right)^{n+1} \frac{P_{n}\left(\mu_{j}\right)}{\mu_{j}^{n} 2^{n+1} n !}
$$


where $P_{n}\left(\mu_{j}\right)=a_{0}+a_{1} \mu_{j}+\cdots+a_{n} \mu_{j}^{n}$ is an $n$-order polynomial in the parameter $\mu_{j}=1+\left(i \omega_{j} \tau\right)^{\alpha}$ which coefficients $a_{k}, 0 \leq k \leq n$ are polynomials in the parameter $\alpha$. Expressions of these coefficients up to order $n=4$ are listed in Appendix 1 . Assuming that the approximation given by Eq. (7.40) is valid, the quality of the solution estimation depends on the number of terms chosen in the series Eq. (7.42). It is sufficient to include a few terms, for instance up to the quadratic order, to achieve a good approximation since the sequence $R_{j}^{(n)}(1) / n$ ! rapidly tends to zero. This statement can intuitively be verified proving $\kappa_{j} / \omega_{j}^{2} \ll 1$, which in turn can be explained using the physical characteristics of the problem that permit to predict the behavior of the mathematical expressions. Calculating the quotient from the previous inequality

$$
\begin{aligned}
\frac{\kappa_{j}}{\omega_{j}^{2}} & =\frac{\phi_{j}^{T} \mathbf{K}_{v} \boldsymbol{\phi}_{j}}{\boldsymbol{\phi}_{j}^{T} \mathbf{K} \phi_{j}}=\frac{\boldsymbol{\phi}_{j}^{T} \mathbf{K}_{v} \boldsymbol{\phi}_{j}}{\boldsymbol{\phi}_{j}^{T} \mathbf{K}_{b} \boldsymbol{\phi}_{j}+\boldsymbol{\phi}_{j}^{T} \mathbf{K}_{v} \boldsymbol{\phi}_{j}} \\
& =\frac{\boldsymbol{\phi}_{j}^{T} \mathbf{K}_{v} \boldsymbol{\phi}_{j}}{\boldsymbol{\phi}_{j}^{T} \mathbf{K}_{b} \boldsymbol{\phi}_{j}}-\left[\frac{\boldsymbol{\phi}_{j}^{T} \mathbf{K}_{v} \boldsymbol{\phi}_{j}}{\boldsymbol{\phi}_{j}^{T} \mathbf{K}_{b} \boldsymbol{\phi}_{j}}\right]^{2}+\left[\frac{\boldsymbol{\phi}_{j}^{T} \mathbf{K}_{v} \boldsymbol{\phi}_{j}}{\boldsymbol{\phi}_{j}^{T} \mathbf{K}_{b} \boldsymbol{\phi}_{j}}\right]^{3}-\cdots
\end{aligned}
$$

The product $\kappa_{j}=\boldsymbol{\phi}_{j}^{T} \mathbf{K}_{v} \boldsymbol{\phi}_{j}$ is directly proportional to the viscoelastic material (usually rubber or rubber-like compounds) rigidity $E_{0}$ and to the section properties $I_{v}, S_{v}$ and $A_{v}$. On the other hand, $\boldsymbol{\phi}_{j}^{T} \mathbf{K}_{b} \boldsymbol{\phi}_{j}$ is proportional to the much higher base material (metallic) $E_{b}, I_{b}, S_{b}, A_{b}$. In general $E_{0} / E_{b} \ll 1$, from which $\boldsymbol{\phi}_{j}^{T} \mathbf{K}_{v} \boldsymbol{\phi}_{j} / \boldsymbol{\phi}_{j}^{T} \mathbf{K}_{b} \boldsymbol{\phi}_{j} \ll 1$ and from Eq. (7.44) it is clear that in fact $\kappa_{j} / \omega_{j}^{2} \ll 1$. Finally, to ensure that the series from Eq. (7.43) is at least $\mathcal{O}\left[\left(\kappa_{j} / \omega_{j}^{2}\right)^{n+1}\right]$, the series $\left\{P_{n}\left(\mu_{j}\right) / \mu_{j}^{n} 2^{n+1} n !\right\}_{n=0}^{\infty}$ must tend to zero or at least be bounded, something that we assume to be true and that will be validated through numerical examples. Substituting the values from Eq. (7.43) into Eq. (7.42) up the quadratic order.

$$
R_{j}(c) \approx-r_{j} \frac{P_{0}\left(\mu_{j}\right)}{2}+r_{j}^{2} \frac{P_{1}\left(\mu_{j}\right)}{4 \mu_{j}}(c-1)-r_{j}^{3} \frac{P_{2}\left(\mu_{j}\right)}{8 \mu_{j}^{2}} \frac{(c-1)^{2}}{2}
$$

where $r_{j}=\frac{\kappa_{j}}{\omega_{j}^{2}} \frac{\mu_{j}-1}{\mu_{j}}$ and

$$
P_{0}\left(\mu_{j}\right)=1, \quad P_{1}\left(\mu_{j}\right)=2\left(\mu_{j}-\alpha\right), \quad P_{2}\left(\mu_{j}\right)=10 \alpha^{2}-\alpha(13+3 \alpha) \mu_{j}+6 \mu_{j}^{2}
$$

With Eq. (7.45), the differential Eq. (7.41) can be integrated obtaining the sought analytical expression for eigenvalues

$$
\lambda_{j}(\alpha, c, \tau) \approx i \omega_{j} \exp \left\{\frac{r_{j}(c-1)}{2}\left[1-\frac{r_{j} P_{1}\left(\mu_{j}\right)}{4 \mu_{j}}(c-1)+\frac{r_{j}^{2} P_{2}\left(\mu_{j}\right)}{12 \mu_{j}^{2}}(c-1)^{2}\right]\right\}
$$

where the exponential term will be noted as $\Delta_{j}(\alpha, c, \tau)$, a dimensionless complex number that depends on the damping parameters through Eqs. (7.46), (7.47). The 
numerical examples will show that the proposed estimate of the eigenvalues given by Eq. (7.47) is very accurate even for parameter values that induce a high damping in the structure. Obviously, to calculate $\lambda_{j}$ it is only necessary to evaluate a function for the particular value of the parameters. From the numerical point of view, the largest computational cost is related to the solution of the undamped eigenvalue problem from Eq. (7.29), necessary to obtain $\omega_{j}$ and $\kappa_{j}$.

\subsubsection{Approximation of the transfer function}

In the previous subsection, the question of how to approximately find the $N$ functions $\lambda_{j}(\alpha, c, \tau) \approx i \omega_{j} \Delta_{j}(\alpha, c, \tau)$ has been answered. For viscoelastic structures with damping exponential functions, [121] showed that an equivalent viscous model can always be found. This model is characterized by its transfer matrix $\mathbf{H}_{\text {eq }}(s)$ that approximates the exact $\mathbf{H}(s)$, defined by Eq. (7.24) and calculated by direct inversion. In addition, the error $\left\|\mathbf{H}(s)-\mathbf{H}_{\mathrm{eq}}(s)\right\|$ depends on the damping level and on the proportionality of the system, i.e., on the relative value of the main diagonal entries of $\boldsymbol{\Gamma}(s)$ with respect to the off-diagonal ones. Calculating $\mathbf{H}_{\mathrm{eq}}(s)$ for viscoelastic materials such as those studied in this article, the approximation will be accurate as long as the assumptions given in [121] are valid. The equivalent transfer matrix will then be given by

$$
\mathbf{H}_{\mathrm{eq}}(s)=\boldsymbol{\Phi} \mathbf{T}(s) \boldsymbol{\Phi}^{T}
$$

$\mathbf{T}(s)=\operatorname{diag}\left[T_{j j}(s)\right]$ is a matrix which entries are the modal transfer functions

$$
T_{j j}(s)=\frac{1}{s^{2}+2 s \Omega_{j} \zeta_{j}+\Omega_{j}^{2}}=\frac{1}{\left(s-\lambda_{j}\right)\left(s-\lambda_{j}^{*}\right)}
$$

and each FRF can be calculated as

$$
H_{i j, \mathrm{eq}}(i \omega)=\sum_{r=1}^{N} \frac{\phi_{i r} \phi_{j r}}{\left(i \omega-\lambda_{j}\right)\left(i \omega-\lambda_{j}^{*}\right)}
$$

where $\phi_{i j}$ are the entries of the modal matrix $\boldsymbol{\Phi}$. The new natural frequencies and modal damping ratios are defined by

$$
\Omega_{j}=\sqrt{\lambda_{j} \lambda_{j}^{*}}=\omega_{j} \sqrt{\Delta_{j} \Delta_{j}^{*}}, \quad \zeta_{j}=-\frac{\Re\left(\lambda_{j}\right)}{\sqrt{\lambda_{j} \lambda_{j}^{*}}}=\frac{\Im\left(\Delta_{j}\right)}{\sqrt{\Delta_{j} \Delta_{j}^{*}}}
$$

In general the effect of rigidity increment with frequency (characteristic of viscoelastic materials) is reflected by the inequality $\Omega_{j}>\omega_{j}$. The matrix $\mathbf{H}_{\mathrm{eq}}(s)$ constructed in this way is related with a viscous model characterized by three dynamic matrices, $\mathbf{M}_{\text {eq }}, \mathbf{C}_{\text {eq }}, \mathbf{K}_{\text {eq }}$, so that

$$
\mathbf{H}_{\mathrm{eq}}(s)=\left(s^{2} \mathbf{M}_{\mathrm{eq}}+s \mathbf{C}_{\mathrm{eq}}+\mathbf{K}_{\mathrm{eq}}\right)^{-1}
$$


The mass matrix $\mathbf{M}_{\mathrm{eq}}$ coincides with the original $\mathbf{M}$. The equivalent $\mathbf{K}_{\mathrm{eq}}$ and $\mathbf{C}_{\text {eq }}$ are constructed so that their transformation into the undamped modal space will produce the natural frequencies $\Omega_{j}$ and modal damping coefficients $2 \Omega_{j} \zeta_{j}$, respectively

$$
\begin{aligned}
\boldsymbol{\Phi}^{T} \mathbf{K}_{\mathrm{eq}} \boldsymbol{\Phi} & =\boldsymbol{\Lambda}_{\mathrm{eq}}=\operatorname{diag}\left[\Omega_{j}^{2}\right] \equiv \mathcal{W}^{\prime} \boldsymbol{\Lambda} \\
\boldsymbol{\Phi}^{T} \mathbf{C}_{\text {eq }} \boldsymbol{\Phi} & =\mathbf{C}_{\text {eq }}^{\prime}=2 \sqrt{\boldsymbol{\Lambda}_{\text {eq }}} \mathcal{Z}=2 \sqrt{\boldsymbol{\Lambda} \mathcal{W}^{\prime}} \mathcal{Z}
\end{aligned}
$$

New matrices $\mathcal{W}^{\prime}(\alpha, c, \tau)=\operatorname{diag}\left[\Delta_{j} \Delta_{j}^{*}\right], \mathcal{Z}(\alpha, c, \tau)=\operatorname{diag}\left[\frac{\Im\left(\Delta_{j}\right)}{\sqrt{\Delta_{j} \Delta_{j}^{*}}}\right]$ have been defined. Note that these matrices depend on the damping parameters through $\Delta_{j}$. Given the modal relations from Eqs. (7.31) and after some matrix operations we obtain

$$
\begin{aligned}
& \mathbf{K}_{\mathrm{eq}}=\mathbf{M} \boldsymbol{\Phi} \mathcal{W}^{\prime} \boldsymbol{\Phi}^{T} \mathbf{K} \equiv \mathcal{W} \mathbf{K} \\
& \mathbf{C}_{\mathrm{eq}}=2 \mathbf{M} \boldsymbol{\Phi} \sqrt{\boldsymbol{\Lambda} \mathcal{W}^{\prime}} \mathcal{Z} \boldsymbol{\Phi}^{T} \mathbf{M}
\end{aligned}
$$

The computation of $\mathbf{K}_{\mathrm{eq}}, \mathbf{C}_{\mathrm{eq}}$ is very efficient since, apart from solving the undamped problem, only requires to find the $N$ values $\Delta_{j}$ defined in Eq. (7.47). This approximation constitutes the main contribution of this article and is a novel and efficient way to address problems involving viscoelastic materials. The resulting model allow us to obtain the response of such problems using the classic tools of modal analysis for viscous systems, both in frequency and time domains. The former is solved through transfer function $\mathbf{H}_{\mathrm{eq}}(s)$ and the latter solving the system of differential equations defined by Eq. (7.25).

\subsection{Numerical examples}

\subsubsection{Example 1: Cantilever beam}

In this example we analyze a cantilever beam made out of steel base material, with $\rho_{b}=7.85 \mathrm{t} / \mathrm{m}^{3}, E_{b}=200 \mathrm{GPa}$, rectangular cross section $50 \times 5 \mathrm{~mm}$ and length $L=500 \mathrm{~mm}$. In the upper surface, a perfectly bonded layer of viscoelastic material with thickness $2 \mathrm{~mm}, \rho_{v}=1.25 \mathrm{t} / \mathrm{m}^{3}$ and static $E_{0}=10 \mathrm{GPa}$. The beam is discretized in ten two-node finite elements with three dof's each, resulting in a mesh with $N=30$ active dof's. Fig. 7.2 shows a drawing of the beam along with their characteristic dimensions and a sketch of the finite element model.

The goal is to validate the approach given by Eq. (7.47) for eigenvalues and the equivalent viscous model constructed from them. A wide range of damping parameter values $\alpha, c, \tau$ is analyzed, covering the vast majority of viscoelastic material applications used for damping and vibration control. The range has been obtained from studies of these materials in references $[11,19-22,24,25,126]$ with the following considerations: 


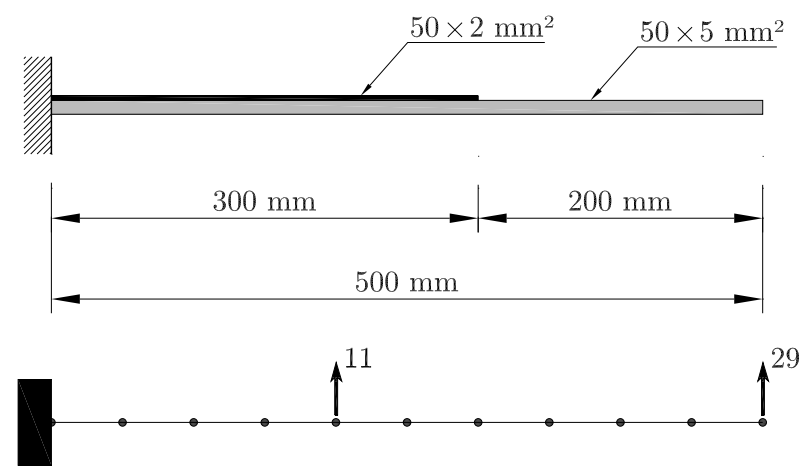

Figure 7.2: Dimensions in $m m$ of beam with base and viscoleastic materials (top) and finite element mesh (bottom). Location of dof's $i, j=11,29$ for FRF

i) The fractional exponent takes values in the range $0 \leq \alpha \leq 1$, considering that physically the vast majority of damping materials are within $0.4 \leq \alpha \leq 0.7$

ii) The range $1 \leq c \leq 10^{4}$ is considered, although generally in practice $10 \leq$ $c \leq 10^{3}$

iii) The relaxation time is a parameter measured in seconds and directly related with frequency $f_{m}(\mathrm{~Hz})$, corresponding to the loss factor peak $\eta_{m}$ from Eq. (7.7), through the equation

$$
\tau=\frac{1}{2 \pi f_{m}} \frac{1}{\sqrt[2 \alpha]{c}}
$$

Looking at the complex modulus graphs from the last references, $f_{m}$ is generally between $10^{2}$ and $10^{6} \mathrm{~Hz}$. The results of the current solution are sensitive to the value of the relaxation time since it is present in Eqs. (7.46) through coefficients $\mu_{j}, r_{j}$; in fact when $\tau \ll 1$ then $\mu_{j} \approx 1$ and $r_{j} \ll 1$, improving the estimated approximation. Since $\tau$ is inversely proportional to $f_{m}$, the representative minimum value $f_{m}=100 \mathrm{~Hz}$ is chosen and the relaxation time is calculated for each $c$ and $\alpha$ with Eq. (7.55). In the numerical results, we can appreciate that even this conservative choice of $\tau$ produces very good results, ensuring that more realistic choices of $f_{m}$ would produce an even more accurate eigenvalue estimate.

In order to verify the efficiency of the eigenvalues' approximation given by Eq. (7.47), the relative error $e_{r}(c, \alpha)$ is calculated. The exact value is calculated using the iterative method of Ruhe [93] based on the transformation of the nonlinear eigenvalue problem given by Eq. (7.26) into a linear one. This transformation is performed through the expansion of the dynamic stiffness matrix $\mathcal{K}(s)$. Starting from an 

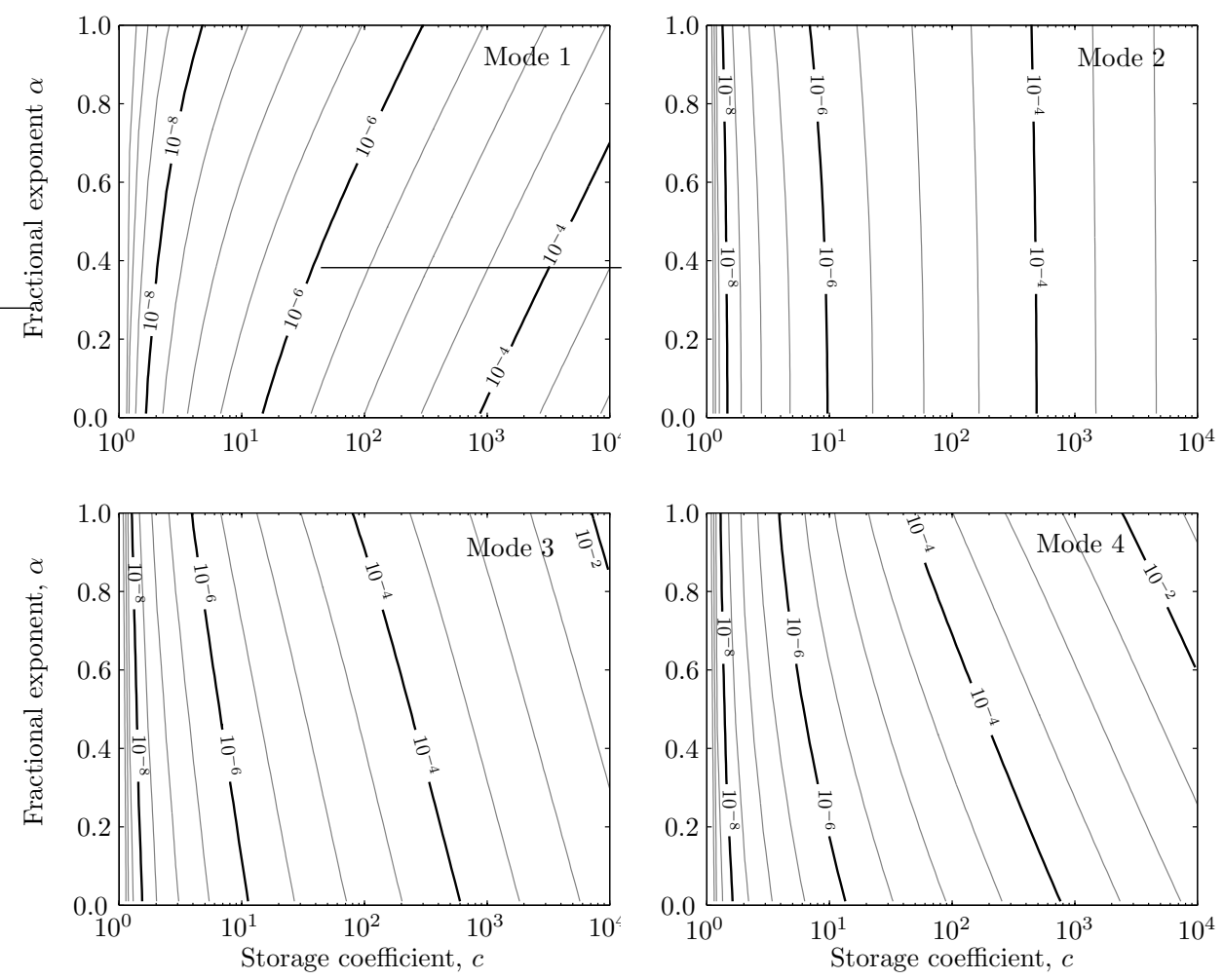

Figure 7.3: Contours of eigenvalues' relative error $e_{j}$ in $\%$ for the first four modes for Example 1.

initial $s_{j}^{(0)}$, we assume that $s_{j}^{(0)}+\delta$ is very close to the $j$ th eigenvalue. Then, it is verified that $\mathcal{K}\left(s_{j}^{(0)}+\delta\right) \mathbf{u}_{j} \approx \mathbf{0}$. Expanding now this matrix up to the first order we obtain

$$
\left[\mathcal{K}\left(s_{j}^{(0)}\right)+\delta \frac{\partial \mathcal{K}\left(s_{j}^{(0)}\right)}{\partial s}\right] \mathbf{u}_{j} \approx \mathbf{0}
$$

This is a linear eigenvalue problem in $\delta$ that does not have to be solved entirely, since only the minimum (in absolute value) eigenvalue $\delta_{j}^{(0)}$ needs to be found; the next iteration can be calculated with $s_{j}^{(1)}=s_{j}^{(0)}+\delta_{j}^{(0)}$. The iterative process consist on constructing the sequence $\left\{s_{j}^{(n)}\right\}$ locally convergent with quadratic speed. The initial guess is taken as the undamped eigenvalue $s_{j}^{(0)}=i \omega_{j}$ and is terminated when the relative error from one iteration to the next is less than $10^{-10}$; the ob- 


\begin{tabular}{ccrrrr} 
Material & Damping & $c$ & $\alpha$ & $\tau(\mathrm{s})$ & $\eta_{m}$ \\
\hline A & Low & $10^{2}$ & 0.357 & $2.516 \times 10^{-6}$ & 0.50 \\
B & Medium & $10^{3}$ & 0.529 & $2.324 \times 10^{-7}$ & 1.00 \\
C & High & $10^{4}$ & 0.636 & $1.141 \times 10^{-8}$ & 1.50 \\
\hline
\end{tabular}

Table 7.1: Damping parameters for three representative viscoelastic materials.

tained solution is considered exact.

Fig. 7.3 represents the relative error $e_{j}(c, \alpha)=100\left|\left(\lambda_{j}-i \omega_{j} \Delta_{j}\right) / \lambda_{j}\right|$ as a function of the damping parameters $c, \alpha$. The results can be considered valid for the majority of the damping viscoelastic materials. It can be appreciated that the error decreases rapidly as $c$ approaches 1 , regardless of the exponent $\alpha$. This is an expected result since the approximated solution is based on an expansion around the starting point $c=1$, where approximate and exact solutions coincide and are equal to the undamped eigenvalues. The error increases as the storage coefficient grows with a slight dependence on the fractional exponent. In some cases the error increases with $\alpha$, such as in the first mode; in other modes the error decreases, as in the third and fourth and finally there is little dependence in the second. Thus, it is not possible to predict a priori an error trend for $\alpha$. In any case the error values are very small, validating the effectiveness of the proposed closed-form expressions.

Now we consider the trend with the mode number for three damping materials and for three different levels. In table 7.1 the parameters are listed, chosen to obtain loss factors to $0.5,1.0$ and 1.5, respectively. As mentioned, the errors are very low with a maximum of $0.01 \%$, see Fig. 7.4. A general increase and oscillation can be appreciated, and the differences in the three levels are in general maintained, with a larger error for higher damping. The exception in the low modes could be due to the variable effect of $\alpha$, depicted in Fig. 7.3. An explanation for the lower error of some modes (for example eighth and tenth) is due to the high proportionality of them; their related damping matrix is more diagonal than that of the rest.

The other important contribution of this paper is the construction of the viscous model defined by $\mathbf{M}, \mathbf{K}_{\mathrm{eq}}, \mathbf{C}_{\mathrm{eq}}$ from Eq.(7.54), directly dependent on the damping parameters $c, \alpha, \tau$. As mentioned, with this new approach we can predict the response of the original viscoelastic model using a much faster algorithm. From Section 7.3.2, the transfer function in the frequency domain can be directly obtained from the expressions of the $N$ eigenvalues. They are given by Eq. (7.47) together with the information of the undamped problem, i.e. the natural frequencies $\omega_{j}$ and real eigenvectors $\phi_{j}$. In Fig. 7.5, the exact (obtained by direct inversion of the dynamic stiffness matrix) and approximated (by Eq. (7.48)) FRF's 


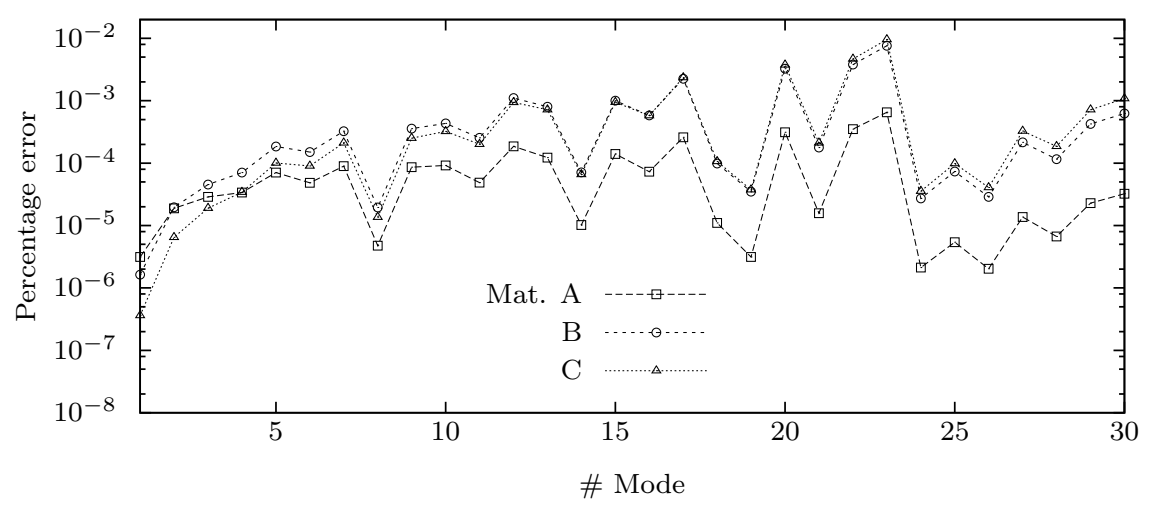

Figure 7.4: Evolution of the relative error with the mode number for Example 1. Parameters in table 7.1

are shown for dof's $i, j=11,29$; see Fig. 7.2 to visualize their position. With the aim to compare the effect of damping layers, the FRF is also shown for a beam without viscoelastic material.

Two effects can be observed in the figure, due to the twofold influence of the complex modulus $\hat{E}_{v}(i \omega)$ from Eq. (7.6). First, the offset of the resonance peaks caused by the first component $E_{d}(\omega)$ and second, the increase of damping with the frequency $\omega$ by the loss factor $\eta(\omega)$. The quality of the approximated FRF is evaluated with the three viscoelastic materials to induce the three damping levels from table 7.1. It is clear that the proposed approach is very accurate specially around the natural frequencies, even in the high frequency range. This is because both models share eigenvalues and, as has been proved, our estimate is of very good quality. If the damping level would be raised, for example by increasing the thickness of the damping material, the error in the evaluation of the FRF would increase as observed in the figures. This effect is expected and was demonstrated in the reference [121].

The largest differences between and current FRF's are found in the antiresonances at $\omega=\{1.9,3.5,5.8,9.1\} \times 10^{4} \mathrm{rad} / \mathrm{s}$; according to the reference they are due to the non-proportionality of the viscoelastic model. For proportionality these differences would significantly be reduced, for instance if the layers are symmetrical and uniformly distributed along the beam. This is due to the viscoelastic and our viscous approach sharing their eigenvectors. 

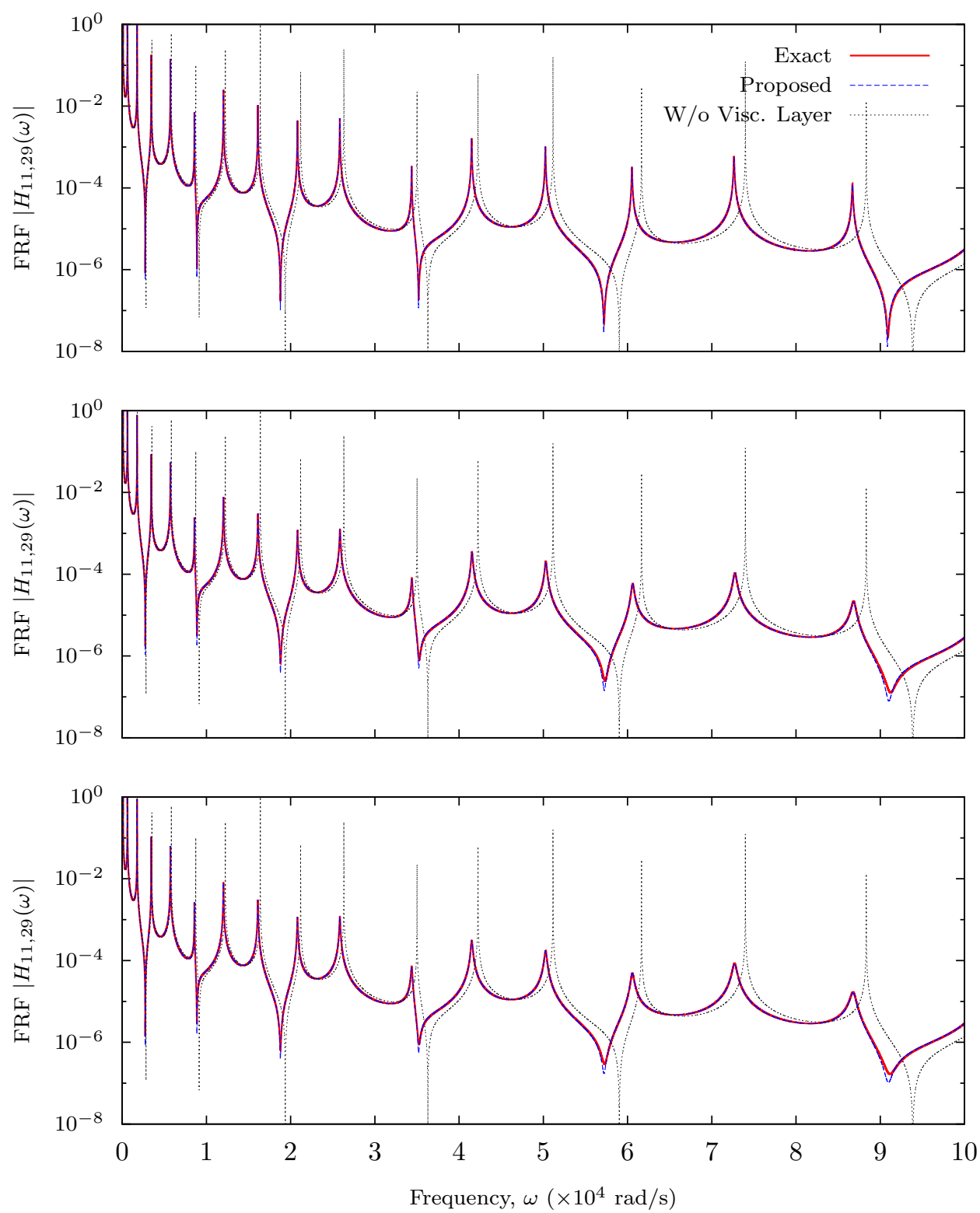

Figure 7.5: Frequency response function at degree-of-fredom numbers $(11,29)$ for Example 1. Exact, proposed (with viscoelastic layer) and exact (without layer) responses. Parameters in table 7.1, top figure for material A, middle for B and bottom for C. 


\subsubsection{Example 2: Frame structure}

This example studies a two-dimensional, framed structure formed by beam elements with layers of viscoelastic material bounded on their upper and lower faces, as shown in Fig. 7.6. The application is limited to the junction areas, forcing the resulting damping matrix not to be proportional. The objective is to validate both the method of eigenvalue calculation and the viscous approximation in more complex structures than that of Example 1. As before, the base material is steel; two section are used: european IPE200 [127] for lintels and HEB200 [128] for columns. The viscoelastic material spans the entire $12 \mathrm{~mm}$ width of the upper and lower flanges with static Young's modulus $E_{0}=50 \mathrm{MPa}$ and density $\rho_{v}=1.25 \mathrm{t} / \mathrm{m}^{3}$. The structure is discretized into 88 finite elements of equal length $L_{e}=0.5 \mathrm{~m}$ forming a mesh of 87 nodes and $N=252$ active dof. Fig. 7.6 sketches the dimensions of the structure and the distribution of the viscoelastic layers.

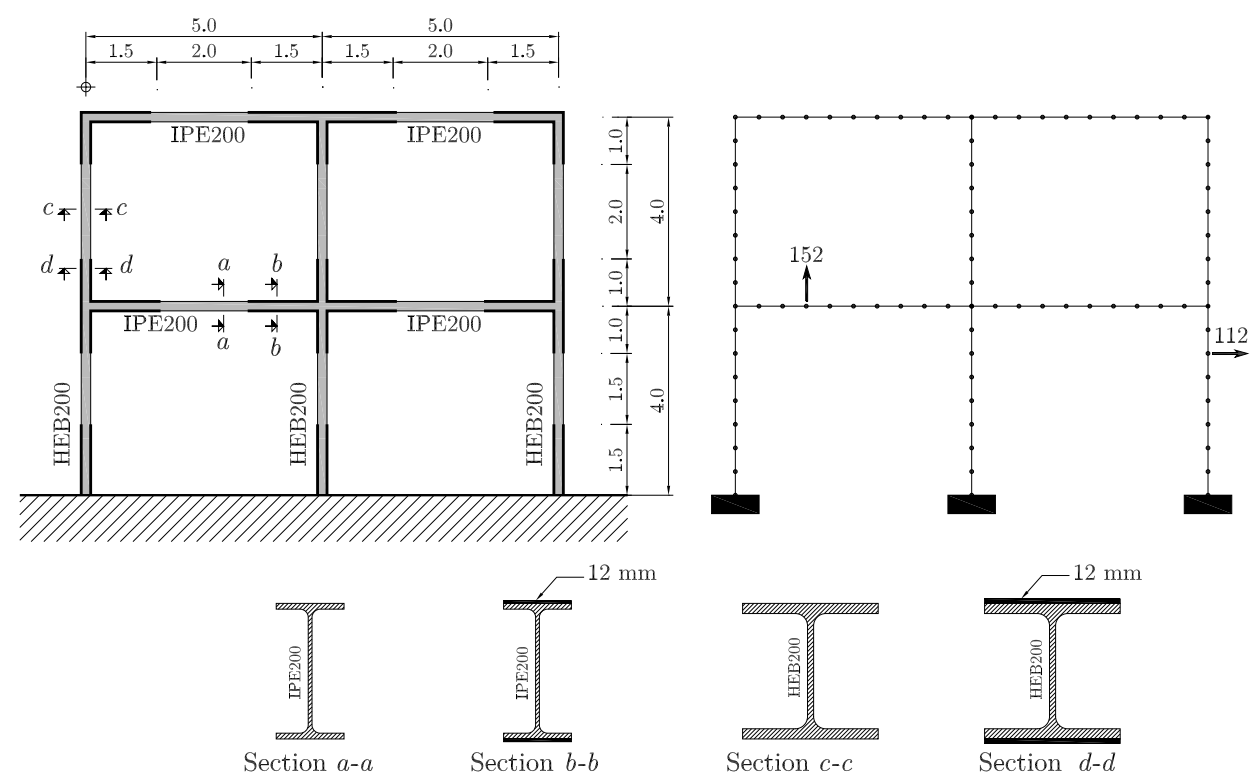

Figure 7.6: Dimensions $(\mathrm{m})$ and distribution of viscoelastic layers. Discretization in finite elements and sections for Example 2 and location of the dof's $i, j=112,152$.

Contour plots equivalent to those of Fig. 7.3 (not shown here) allow us to draw the same conclusions that were given in Example 1. Using the same damping materials A, B, C as before, in Fig. 7.7 the evolution of the relative error with the structural mode number is shown. It should first be noted that the trend is upward but with very low slope so that any mode presents an error above $2 \%$. Furthermore, 
in general the errors obtained using the low damped material A are lower than those related to the high damping material C. However, in some cases the behavior reverses, due to the different values of the fractional exponent $\alpha$ and relaxation time $\tau$. It is also noted that after the 50th mode, some errors increase rapidly from the average trend, since these modes exhibit a larger lack of proportionality. Therefore their associated eigenvalues are more affected by the off-diagonal entries of $\boldsymbol{\Gamma}\left(\lambda_{j}, c\right)$ that consequently propagate errors.

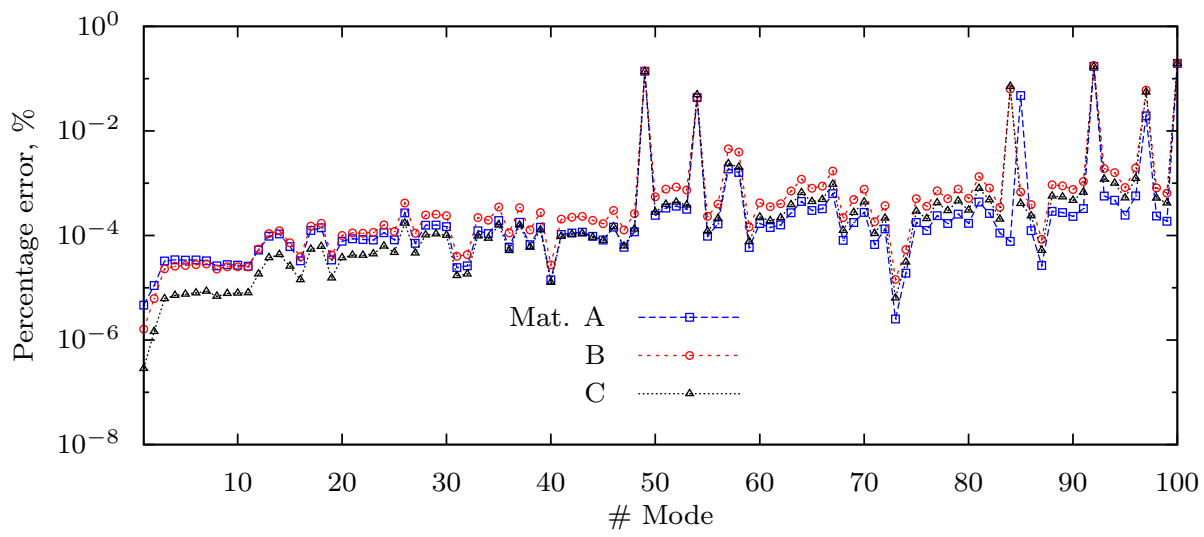

Figure 7.7: Evolution of relative error with the mode number for three materials (see table 7.1) and Example 2.

Finally, in Fig. 7.8 the FRF associated with dof's $i, j=112,152$ (marked in Fig. 7.6) are plotted. These FRF's are accurately calculated by direct inversion and approximately with the proposed method based on Eq. (7.48). It is clear that both distributions fit very well. The effect of the damping layers is more important in high frequencies, for which the complex modulus (loss factor as well as Young's modulus) take important values. The approximation given by the FRF is slightly poorer for high frequencies than for low, see Fig. 7.7. This fact is specially clear for materials $\mathrm{B}$ and $\mathrm{C}$ in the range $2.5 \times 10^{4}<\omega<5 \times 10^{4} \mathrm{rad} / \mathrm{s}$, where the structure response is more damped. But even in these cases, the highly accurate adjustment between both curves is remarkable. Inspection of entries of the receptance matrix different from the presented dof's (that is, other FRF's) allow us to affirm that the estimation is also of very good quality. This observation partially validates the method as an efficient tool for the analysis of this structural type. 

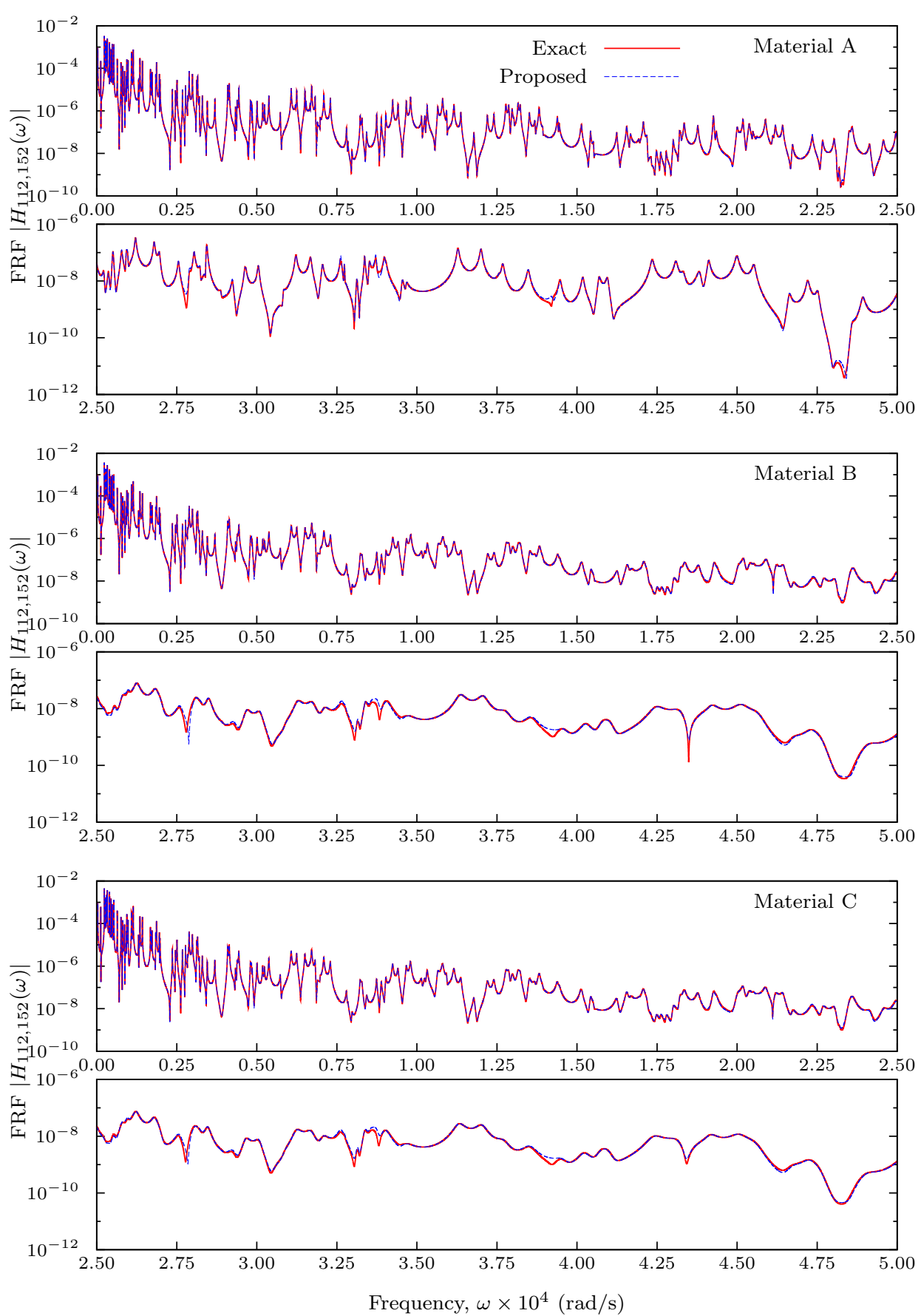

Figure 7.8: Frequency Response Function at dof's $i, j=112,152$ for Example 2. 


\subsection{Conclusions}

In this paper we propose a new method for the analysis of beams and framed structures, damped with free layers of viscoelastic material which constitutive model is based on the fractional derivative. The equations of motion are obtained using the finite element method, which is developed from variational principles in the frequency domain. A frequency-dependent damping matrix is formulated, resulting in free motion equations that represent a nonlinear problem of eigenvalues. The main contribution of this work is to obtain, assuming the hypothesis of proportional damping, approximated eigenvalue solutions in closed-form. These forms are developed as function of the damping parameters, for which solutions of the linear eigenvalue problems are not required except for the undamped counterpart; in addition iterative procedures are not necessary.

The availability of analytical expressions for complex eigenvalues given by the current method permits the direct application of an equivalent viscous model previously developed by the authors. The procedure is, on one side, to obtain the transfer function of a viscous model, and on the other equivalent stiffness and damping matrices in closed form. The matrices are function of the damping parameters and of the original mass and stiffness matrices. Since the current viscous model accurately duplicates the response of a viscoelastic model, standard tools developed for general viscous damping can be used with much lower computational cost.

In order to validate the proposed method, two numerical examples are presented. The first, simulates a cantilever beam with a viscoelastic layer bonded to the upper face. The second, analyzes a two-dimensional metallic frame composed of beams and columns also with bonded layers but located close to the intersections. For both examples, the relative error between the complex eigenvalues from the proposed explicit expressions and the exact ones, calculated by iteration, is presented. The results indicate that these errors are very small for a wide range of damping parameters, within which most damping materials lay. The errors show a slight upward trend with the mode number but maintain a very small value, with very good accuracy of the frequency response function even for high frequencies.

Although this work has been developed for beams, the methodology can be extended to plates with free viscoelastic layers, as long as the adopted assumptions are valid. At present, the authors are conducting research related to other viscoelastic structures such as those with constrained viscoelastic layers or with viscoelastic dampers. 


\section{Appendix 7.A Calculation of $R_{j}^{(n)}(1)$}

We porpose to obtain an expression of $n$th derivative of $R_{j}(c)=\mathcal{R}_{j}\left(\lambda_{j, \operatorname{lin}}(c), c\right)$ evalated at $c=1$ and for $n \geq 0$. The analytical expression of $\mathcal{R}_{j}(s, c)$ is available form Eq. (7.38). In addition, $\lambda_{j, \operatorname{lin}}(c)$ is a linear expression in $c$ by definition. With these considerations we can take derivatives in $R_{j}(c)$ by using the chain rule, resulting

$$
\begin{aligned}
R_{j}^{\prime}(c)=\frac{\mathrm{d} R_{j}}{\mathrm{~d} c} & =\left[\left.\frac{\partial \mathcal{R}_{j}}{\partial s}\right|_{s=\lambda_{j, \operatorname{lin}}(c)}\right] \lambda_{j, \operatorname{lin}}^{\prime}(c)+\left.\frac{\partial \mathcal{R}_{j}}{\partial c}\right|_{s=\lambda_{j, \operatorname{lin}}(c)} \\
R_{j}^{\prime \prime}(c)=\frac{\mathrm{d}^{2} R_{j}}{\mathrm{~d} c^{2}} & =\left[\left.\frac{\partial^{2} \mathcal{R}_{j}}{\partial s^{2}}\right|_{s=\lambda_{j, \operatorname{lin}}(c)} \lambda_{j, \operatorname{lin}}^{\prime}(c)+\left.\frac{\partial^{2} \mathcal{R}_{j}}{\partial c \partial s}\right|_{s=\lambda_{j, \operatorname{lin}}(c)}\right] \lambda_{j, \operatorname{lin}}^{\prime}(c) \\
& +\left.\frac{\partial^{2} \mathcal{R}_{j}}{\partial c^{2}}\right|_{s=\lambda_{j, \operatorname{lin}}(c)}
\end{aligned}
$$

Higher order derivatives can be calculated following the same procedure taking into account that $\lambda_{j \text {,lin }}^{\prime \prime}(c)=0$. Thus, after several simplifications the expressions of $R_{j}^{(n)}(1)$ for $0 \leq n \leq 4$ are

$$
\begin{aligned}
& R_{j}(1)=-\left(\frac{\mu_{j}-1}{\mu_{j}}\right)\left(\frac{\kappa_{j}}{\omega_{j}^{2}}\right) \frac{P_{0}\left(\mu_{j}\right)}{2} \\
& R_{j}^{\prime}(1)=+\left(\frac{\mu_{j}-1}{\mu_{j}}\right)^{2}\left(\frac{\kappa_{j}}{\omega_{j}^{2}}\right)^{2} \frac{P_{1}\left(\mu_{j}\right)}{4 \mu_{j}} \\
& R_{j}^{\prime \prime}(1)=-\left(\frac{\mu_{j}-1}{\mu_{j}}\right)^{3}\left(\frac{\kappa_{j}}{\omega_{j}^{2}}\right)^{3} \frac{P_{2}\left(\mu_{j}\right)}{8 \mu_{j}^{2}} \\
& R_{j}^{\prime \prime \prime}(1)=+\left(\frac{\mu_{j}-1}{\mu_{j}}\right)^{4}\left(\frac{\kappa_{j}}{\omega_{j}^{2}}\right)^{4} \frac{P_{3}\left(\mu_{j}\right)}{16 \mu_{j}^{3}} \\
& R_{j}^{\prime \prime \prime \prime}(1)=-\left(\frac{\mu_{j}-1}{\mu_{j}}\right)^{5}\left(\frac{\kappa_{j}}{\omega_{j}^{2}}\right)^{5} \frac{P_{4}\left(\mu_{j}\right)}{32 \mu_{j}^{4}}
\end{aligned}
$$


where $\mu_{j}=1+\left(i \omega_{j} \tau\right)^{\alpha}$ and

$$
\begin{aligned}
P_{0}\left(\mu_{j}\right) & =1 \\
P_{1}\left(\mu_{j}\right) & =2\left(\mu_{j}-\alpha\right) \\
P_{2}\left(\mu_{j}\right) & =10 \alpha^{2}-\alpha(13+3 \alpha) \mu_{j}+6 \mu_{j}^{2} \\
P_{3}\left(\mu_{j}\right) & =-78 \alpha^{3}+45 \alpha^{2}(3+\alpha) \mu_{j}-2 \alpha\left(43+18 \alpha+2 \alpha^{2}\right) \mu_{j}^{2}+24 \mu_{j}^{3} \\
P_{4}\left(\mu_{j}\right) & =816 \alpha^{4}-24 \alpha^{3}(74+29 \alpha) \mu_{j}+2 \alpha^{2}\left(767+468 \alpha+73 \alpha^{2}\right) \mu_{j}^{2} \\
& -\left(634 \alpha+367 \alpha^{2}+74 \alpha^{3}+5 \alpha^{3}\right) \mu_{j}^{3}+120 \mu_{j}^{4}
\end{aligned}
$$

are polynomials in $\mu_{j}$ which coefficients depend on the fractional exponent $\alpha$. The mathematical form of $R_{j}^{(n)}(1)$ for the first four derivatives given in Eq. (7.58) allow us to predict the general form as

$$
R_{j}^{(n)}(1)=\left.\frac{1}{n !} \frac{\partial^{n} R_{j}}{\partial c^{n}}\right|_{c=1}=\frac{(-1)^{n+1}}{\mu_{j}^{n} 2^{n+1}}\left(\frac{\mu_{j}-1}{\mu_{j}}\right)^{n+1}\left(\frac{\kappa_{j}}{\omega_{j}^{2}}\right)^{n+1} P_{n}\left(\mu_{j}\right)
$$

where the polynomials $P_{n}\left(\mu_{j}\right)$ must be obtained by recursion from the previous derivatives. 



\section{Bibliography}

[7] M.A. Biot. "Variational Principles in Irreversible Thermodynamics with Application to Viscoelasticity." In: Physical Review 97.6 (1955), 1463-1469 (cit. on pp. 3, 20, 24, 37, 58, 82, 93, 192).

[11] A.D. Nashif, D.I.G. Jones, and J.P. Henderson. Vibration Damping. John Wiley and Sons, 1985 (cit. on pp. 3, 45, 160, 192, 194, 206).

[12] David I.G. Jones. Handbook of viscoelastic vibration damping. John Wiley \& Sons, 2001 (cit. on pp. 3, 45, 160, 192, 194).

[13] C.T. Sun and Y.P. Lu. Vibration Damping of Structural Elements. Prentice Hall PTR, 1995 (cit. on pp. 3, 192, 194).

[14] D.F. Golla and P.C. Hughes. "Dynamics of Viscoelastic Structures - A Time-domain, Finite-element Formulation." In: Journal of Applied MechanicsTransactions of the ASME 52.4 (1985), 897-906 (cit. on pp. 3, 20, 22, 24, $58,60,82,84,118,160,162,164,192)$.

[15] R.L. Bagley and P.J. Torvik. "A Theoretical Basis for the Application of Fractional Calculus to Viscoelasticity." In: Journal of Rheology 27.3 (1983), 201-210 (cit. on pp. 4, 20, 82, 192, 196).

[16] R.L. Bagley and P.J. Torvik. "Fractional Calculus - A Different Approach to the Analysis of Viscoelastically Damped Structures." In: AIAA Journal 21.5 (1983), 741-748 (cit. on pp. 4, 20, 24, 82, 192, 193, 196).

[18] R.L. Bagley and P.J. Torvik. "Fractional Calculus in the Transient Analysis of Viscoelastically Damped Structures." In: AIA A journal 23.6 (1985), 918 925 (cit. on pp. 4, 193).

[19] R.L. Bagley and P.J. Torvik. "On the Fractional Calculus Model of Viscoelastic Behavior." In: Journal of Rheology 30.1 (1986), 133-155 (cit. on pp. 4, 196, 206).

[20] T. Pritz. "Analysis of four-parameter fractional derivative model of real solid materials." In: Journal of Sound and Vibration 195.1 (1996), 103-115 (cit. on pp. 4, 42, 45, 150, 192, 196, 206). 
[21] T. Pritz. "Frequency dependences of complex moduli and complex Posson's ratio of real solid materials." In: Journal of Sound and Vibration 214.1 (1998), 83-104 (cit. on pp. 4, 45, 150, 206).

[22] T Pritz. "Loss factor peak of viscoelastic materials: Magnitude to width relations." In: Journal of Sound and Vibration 246.2 (2001), 265-280 (cit. on pp. 4, 45, 150, 196, 206).

[23] T Pritz. "Five-parameter fractional derivative model for polymeric damping materials." In: Journal of Sound and Vibration 265.5 (2003), 935-952 (cit. on pp. 4,192$)$.

[24] T. Pritz. "The Poisson's loss factor of solid viscoelastic materials." In: Journal of Sound and Vibration 306.3-5 (2007), 790-802 (cit. on pp. 4, 206).

[25] T. Pritz. "Relation of bulk to shear loss factor of solid viscoelastic materials." In: Journal of Sound and Vibration 324.3-5 (2009), 514-519 (cit. on pp. 4, 206).

[28] S. Adhikari. "Dynamics of Non-viscously Damped Linear Systems." In: Journal of Engineering Mechanics 128.3 (2002), 328-339 (cit. on pp. 4-6, 22, 35, 36, 58, 84, 94, 121, 125, 129, 131, 160-162, 165, 168, 170, 171, 203).

[31] Sondipon Adhikari and Blanca Pascual. "Eigenvalues of linear viscoelastic systems." In: Journal of Sound and Vibration 325.4-5 (2009), 1000-1011 (cit. on pp. 5, 21, 35, 41, 42, 69, 70, 76, 83, 119, 120, 141, 151, 152, 193).

[32] Sondipon Adhikari and Blanca Pascual. "Iterative Methods for Eigenvalues of Viscoelastic Systems." In: Journal of Vibration and Acoustics 133.2 (2011), pp. 021002.1-021002.7 (cit. on pp. 5, 21, 35, 42, 59, 83, 108, 119, $171,193)$.

[33] M. Lázaro, J. L. Pérez-Aparicio, and M. Epstein. "Computation of eigenvalues in proportionally damped viscoelastic structures based on the fixedpoint iteration." In: Applied Mathematics and Computation 219.8 (2012), 3511-3529 (cit. on pp. 7, 59, 119, 193).

[34] M. Lázaro and J. L. Pérez-Aparicio. "Multiparametric computation of eigenvalues for linear viscoelastic structures." In: Computers \& Structures 117.0 (2013), pp. $67-81$ (cit. on pp. 10, 21, 194, 201).

[46] Wei H. Yang. "A method for eigenvalues of sparse $\lambda$-matrices." In: International Journal for Numerical Methods in Engineering 19.6 (1983), pp. 943948 (cit. on pp. 21, 83, 118, 193).

[54] A Muravyov and SG Hutton. "Closed-form solutions and the eigenvalue problem for vibration of discrete viscoelastic systems." In: Journal of Applied Mechanics-Transactions of the ASME 64.3 (1997), 684-691 (cit. on pp. 21, 58, 83, 119, 160, 164, 193). 
[56] S. Menon and J. Tang. "A state-space approach for the dynamic analysis of viscoelastic systems." In: Computers \& Structures 82.15-16 (2004), pp. 1123 -1130 (cit. on pp. 21, 83, 119, 160, 164, 193).

[63] M. Lázaro and J. L. Pérez-Aparicio. "Parametric solutions of the eigenvalue problem for single degree-of-freedom viscoelastic systems." In: AIAA Journal. 0 (2013), pp. i-xvii (cit. on pp. 21, 59, 69, 70, 72, 75, 76, 193, 201).

[71] S. Adhikari and J. Woodhouse. "Quantification of non-viscous damping in discrete linear systems." In: Journal of Sound and Vibration 260.3 (2003), pp. 499-518 (cit. on pp. 33, 88, 94, 125, 197, 203).

[92] H. Oberst and K. Frankenfeld. "Über die Dämpfung der Biegeschwingungen Dünner Bleche Durch Fest Haftende Belage." In: J. Acustica 2 (1952), pp. 181-194 (cit. on pp. 150, 192, 194).

[93] A Ruhe. "Algorithms for Nonlinear Eigenvalue Problem." In: SIAM Journal on Numerical Analysis 10.4 (1973), 674-689 (cit. on pp. 151, 193, 207).

[95] J. P. Bandstra. "Comparison Of Equivalent Viscous Damping and Nonlinear Damping in Discrete and Continuous Vibrating Systems." In: Journal of Vibration, Acoustics, Stress and Reliability in Design 105 (1983), 382392 (cit. on pp. 160, 193).

[98] Eric E. Ungar and Jr. Edward M. Kerwin. "Loss Factors of Viscoelastic Systems in Terms of Energy Concepts." In: The Journal of the Acoustical Society of America 34.7 (1962), pp. 954-957 (cit. on pp. 161, 193).

[103] D.J. Segalman. "Calculation of Damping Matrices for Linearly Viscoelastic Structures." In: Journal of Applied Mechanics 54.3 (1987), 585-588 (cit. on pp. 161, 193).

[104] Sondipon Adhikari. "A Reduced Second-Order Approach for Linear Viscoelastic Oscillators." In: Journal of Applied Mechanics-Transactions of the ASME 77.4 (2010), pp. 1-8 (cit. on pp. 161, 193).

[106] C.M.A. Vasques, R.A.S. Moreira, and J. Dias Rodrigues. "Viscoelastic Damping Technologies-Part I: Modeling and Finite Element Implementation." In: Journal of Advanced Research in Mechanical Engineering 1.2 (2010), 76-95 (cit. on p. 192).

[107] F. Cortés and Maria Jesús Elejabarrieta. "Structural vibration of flexural beams with thick unconstrained layer damping." In: International Journal of Solids and Structures 45.22-23 (2008), 5805-5813 (cit. on p. 192).

[108] Peter J. Torvik. "Analysis of free-layer damping coatings." In: Layered, Functional Gradient Ceramics, and Thermal Barrier Coatings: Design, Fabrication and Applications. Vol. 333. 2007, 195-214 (cit. on p. 192). 
[109] YC Xu and A Nashif. "Measurement, analysis and modeling of the dynamic properties of materials." In: Sound and Vibration 30.7 (1996), 20-23 (cit. on p. 192).

[110] ASTM E 756-04. Standard Test Method for Measuring Vibration-Damping Properties of Materials. American Society for Testing and Material, 2004 (cit. on p. 193).

[111] MD Rao. "Recent applications of viscoelastic damping for noise control in automobiles and commercial airplanes." In: Journal of Sound and Vibration 262.3 (2003), 457-474 (cit. on p. 193).

[112] J Kim, J Ryu, and L Chung. "Seismic performance of structures connected by viscoelastic dampers." In: Engineering Structures 28.2 (2006), 183-195 (cit. on p. 193).

[113] KL Shen and TT Soong. "Modeling of Viscoelastic Dampers for Structural Applications." In: Journal of Engineering Mechanics-ASCE 121.6 (1995), 694-701 (cit. on p. 193).

[114] KL Shen et al. "Seismic Behavior of Reinforced-concrete Frame with Added Viscoelastic Dampers." In: Engineering Structures 17.5 (1995), 372-380 (cit. on p. 193).

[115] YL Xu and WS Zhang. "Modal analysis and seismic response of steel frames with connection dampers." In: Engineering Structures 23.4 (2001), 385-396 (cit. on p. 193).

[116] SY Hsu and A Fafitis. "Seismic Analysis Design of Frames with Viscoelastic Connections." In: Journal of Structural Engineering-ASCE 118.9 (1992), 2459-2474 (cit. on p. 193).

[117] Kyung-Won Min, Jinkoo Kim, and Sang-Hyun Lee. "Vibration tests of 5storey steel frame with viscoelastic dampers." In: Engineering Structures 26.6 (2004), pp. $831-839$ (cit. on p. 193).

[118] Roman Lewandowski and Zdzislaw Pawlak. "Dynamic analysis of frames with viscoelastic dampers modelled by rheological models with fractional derivatives." In: Journal of Sound and Vibration 330.5 (2011), 923-936 (cit. on p. 193).

[119] E. Moliner, P. Museros, and M. D. Martinez-Rodrigo. "Retrofit of existing railway bridges of short to medium spans for high-speed traffic using viscoelastic dampers." In: Engineering Structures 40 (2012), 519-528 (cit. on p. 193).

[120] Sang-Hyun Lee et al. "Evaluation of equivalent damping ratio of a structure with added dampers." In: Engineering Structures 26.3 (2004), pp. 335 -346 (cit. on p. 193). 
[121] M. Lázaro, J.L. Pérez-Aparicio, and M. Epstein. "An Equivalent Viscous Model for Linear Viscoelastic Systems." In: Mechanical System and Signal Processing 1 (2013), pp. i-xviii (cit. on pp. 193, 194, 205, 210).

[122] S. Gopalakrishnan and J.F. Doyle. "Wave-propagation in Connected Waveguides of Varying Cross-section." In: Journal of Sound and Vibration 175.3 (1994), 347-363 (cit. on pp. 194, 197).

[123] James F. Doyle. Wave Propagation in Structures. Spectral Analysis Using Fast Discrete Fourer Transforms. Springer, 1997 (cit. on pp. 194, 197).

[124] Y. Liu et al. "Theoretical study of two-dimensional phononic crystals with viscoelasticity based on fractional derivative models." In: Journal of Physics D: Applied Physics 41.6 (2008), pp. 1-7 (cit. on p. 196).

[125] Matlab and Simulink (C)2012 The Mathworks, Inc. (Cit. on p. 200).

[126] Michael L. Drake. Damping properties of various materials. Tech. Report AFWAL-TR-88-4248. Tech. rep. Air Force Wright Aeronautical Laboratories. Material Laboratory (AFWAL-MLLN), 1989 (cit. on p. 206).

[127] Dimensions of Europan I-Beams. Standard EU 19-5\%. Standard EU 19-57 (cit. on p. 212).

[128] Dimensions of European wide flange beams. Standard EU 53-62. Standard EU 53-62 (cit. on p. 212). 



\section{8}

\section{Conclusions}

\subsection{Summary}

The damping models of viscoelastic nature are characterized by dissipative forces that depend on the history of the velocity response via convolution integrals with hereditary kernels. The dynamic equilibrium leads to a system of integrodiferential equations. In the frequency domain, the free motion equations result in a nonlinear eigenvalue problem due to the presence of a frequency-dependent damping matrix.

In this thesis, two objectives have been searched: (i) to advance in the knowledge of the nonlinear eigenproblem of viscoelastically damped structures and (ii) to propose new methods for the frequency-domain response by means of the development of the equivalent viscous model.

The contributions are organized in six chapters; each one corresponds with an article submitted to indexed journals included in the Journal Citations Reports. Two papers have already been published and the other four are currently under review. The original ideas developed in each chapter are now detailed.

In Chapter 2 a new iterative method to compute the eigenvalues for proportional or lightly non-proportional viscoelastic systems is developed. The key idea is the construction of two complex functions that converge up to the conjugate complex 
in a fixed-point iterative scheme. In addition, the convergence is rigorously analyzed in three theorems that form the theoretical body of the paper and where two important results are proved: (1) the method demonstrates to be of great robustness, i.e. the sequences always converge, independently on the chosen initial point; and (2) there exists a close relationship between the convergence velocity, the damping level and the viscoealsticity of the damping model. It is demonstrated that the lighter the damping the faster the convergence.

In Chapter 3 new contributions on the non-viscous eigenvalues of viscoelastic oscillators are presented. The starting point is the proof of a new property that characterizes to these eigenvalues leading to a new concept: the non-viscous set. This set, formed by an union of closed intervals, is located within the negative real numbers and contains all non-viscous eigenvalues. The limits of the intervals can be analytically calculated for exponentially damped functions up to four kernels, although only the cases of one and two kernels is developed. For the general case of $N$ kernels approximated closed-forms using an asymptotic representation of the non-viscous eigenvalues are proposed. Another important contribution is the use of this property to the development of new analytical approaches of the non-viscous eigenvalues. The method is successfully validated by comparison with other methods available in the literature.

In Chapter 4 the so-called parametric method for the numerical computation of eigenvalues in single dof is proposed. The damping function of a viscoelastic oscillator is the mathematical entity that characterizes the energy dissipation, either in time-domain or in frequency domain. In general this function in frequency domain depends on the Laplace eigenparameter and on several damping parameters. The key idea of this method is to consider the damping function variable also in one of this parameters. The eigenvalues are transformed into unknown functions; the perturbation techniques together with the eigensensitivities lead to approximate the eigenvalues as solutions of certain ordinary differential equation in separated variables. Presented the algorithm, the approximation order of the proposed approaches is derived and validated by several numerical examples covering the exponentially damped systems.

In Chapter 5 the parametric method is generalized to cover the case of multiple dof systems with a damping matrix assumed variable in several parameters. The eigensolutions are consequently multivariable functions of certain multiparametric array. First order solutions by perturbation asymptotic techniques of the parameters are developed. Using the expression of the eigenvalue derivative of non-proportional damping systems, the approximations are constructed as solutions of certain ordinary differential equation. A remarkable difference respect to the method for single dof is the eigenvectors' approximation; for it an alternative method based on the solution of the linear eigenproblem of the dynamic stiffness matrix is developed. The improvement of the order of approximation respect to that of the first order perturbation is mathematically demonstrated. Moreover, 
the method is checked by means of several numerical examples, discrete and continuous, using also different damping materials.

In Chapter 6 the equivalent viscous model is developed. This approach is conceived as an alternative method to obtain the response of systems with viscoelastic damping. The starting point is the exact transfer function of a viscoelastic system. As known, a closed-form expression can be obtained from the pole-residue expansion. Through several transformations, the transfer function is be decomposed as the sum of the transfer function of a viscous model plus a residual term. The latter depends on some features of the damping matrix: (1) on the proportionality, i.e. the modal decoupling capability, (2) on the level of damping or how high in absolute value the entrees of the damping matrix are and (3) on the viscoelasticity or how high the entrees of the damping matrix's derivative respect to the Laplace eigenparameter are. The developments lead to new damping and stiffness matrices that configure the new viscous model. The response of this new model is under certain conditions a good approximation of the exact one.

In Chapter 7 the equivalent viscous model is used for the analysis of frame structures with unconstrained viscoelastic layers. For the time-domain constitutive relationships of the damping materials, a four-parameter fractional-derivative based model is considered. With help of the finite element method, the motion equations in frequency domain are derived. As a result a damping matrix that depends on the frequency and on the damping parameters is obtained. According to the proposed parametric method, one of these parameters - the storage coefficientis assumed variable. It is demonstrated that the eigenvalues' sensitivities, considered as functions of the chosen parameter, result very regular and smooth. Thus, after some transformations, closed-forms of the eigenvalues are developed. This is traduced in a very low computational effort for their calculation. In the numerical examples the method accuracy can be visualized and the transfer functions compared. Very good agreement between proposed and exact solution is observed, even for higher modes in the high-frequencies range.

\subsection{Recommendations for further work}

The contributions of this thesis may lead to new research; some new ideas have already a solid theoretical foundation and they could be expressed in paper format. Others, however, are under study and should be developed further. A summary of the proposals for the future work is listed below

- The proposed iterative method can efficiently be used to compute complex eigenvalues in proportional or lightly non-proportional systems. The question arises whether a generalization of this method can be constructed for 
strongly non-proportional systems. Intuitively it seems that the complex eigenvectors would play an important role in this method.

- Some research has already been published in the literature about overcritically damped systems, specially for exponentially damped oscillators with one kernel. The so-called overdamped region, that is the set of damping parameters that leads to real eigenvalues, have not been defined for two or more exponential kernels. This is an interesting research area and some advances have been carried out by the author in this direction.

- The proposed multiparameteric method is based on the first order perturbation of the parametric vector. The question is: why not construct a second order perturbation in terms of the parametric vector? This question was in fact asked by a reviewer of this paper. Since the eigenvalues are multivariable functions of the parameters, the quadratic term of the Taylor expansion must be well defined and for that the Hessian matrix. which defines the cross derivatives respect to the parameters, should be symmetric. Thus the question could be reformulated as: under what conditions the Hessian matrix is symmetric?

- The main contributions of this thesis have been developed within the frequency domain. In fact, the numerical methods for the nonlinear eigenproblem covers a large part of it. The main disadvantage arises for systems with a large number of dof; in them the extraction of all eigenvalues and eigenvectors, included those non-viscous, can result considerably expensive in terms of computational effort. This is one of the reasons to encourage the timedomain methods, which directly integrate the system of integro-differential equations. Several methods have been proposed for different viscoelastic models but according to the author, new approaches may still be proposed.

- In this thesis two methods to compute non-viscous eigenvalues have been proposed, both developed under the assumption of proportional or lightly non-proportional damping. A challenge for further work is the research of new numerical approaches for the calculation of these eigenvalues but covering strongly non-proportional damping.

- The equivalent viscous model proposes new stiffens and damping matrices. However, the mass matrix remains the same as that of the original model. New research could be oriented in the future to modify the entrees of the mass matrix in order to improve the approximation.

- Experimentation in real structures is always a field on constant development. The robustness of a numerical method must be tested in realistic engineering applications such as for instance damping identification, experimental modal analysis, new energy dissipation devices and dampers, vibration control or structural health monitoring. Undoubtedly new research lines should go in this direction to consolidate the proposed methodologies. 